\title{
Kinetik der radikalischen \\ Polymerisation von Monomeren \\ mit mesogener Seitengruppe in \\ isotroper und anisotroper Lösung
}

\author{
Dissertation \\ zur Erlangung des mathematisch-naturwissenschaftlichen Doktorgrades \\ "Doctor rerum naturalium" \\ der Georg-August-Universität Göttingen \\ im Promotionsprogramm Chemie \\ der Georg-August University School of Science (GAUSS)
}

vorgelegt von

Alex Groschopp

aus Buenos Aires

Göttingen, 2018 


\section{Betreuungsausschuss}

Prof. Dr. Philipp Vana, MBA Institut für Physikalische Chemie Georg-August-Universität Göttingen

Prof. Dr. Martin Suhm

Institut für Physikalische Chemie Georg-August-Universität Göttingen

\section{Mitglieder der Prüfungskommission}

\section{Referent}

Prof. Dr. Philipp Vana, MBA Institut für Physikalische Chemie Georg-August-Universität Göttingen

\section{Korreferent}

Prof. Dr. Martin Suhm

Institut für Physikalische Chemie Georg-August-Universität Göttingen

\section{Weitere Mitglieder der Prüfungskommission}

Prof. Dr. Ricardo Mata Institut für Physikalische Chemie Georg-August-Universität Göttingen

Prof. Dr. Dietmar Stalke Institut für Anorganische Chemie Georg-August-Universität Göttingen

PD Dr. Thomas Zeuch Institut für Physikalische Chemie Georg-August-Universität Göttingen

Dr. Florian Ehlers

Institut für Physikalische Chemie Georg-August-Universität Göttingen

Tag der mündlichen Prüfung: 05. Februar 2018 


\section{Inhaltsverzeichnis}

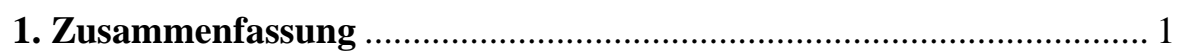

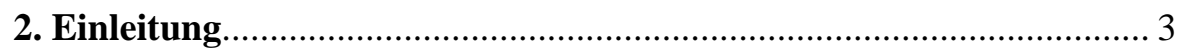

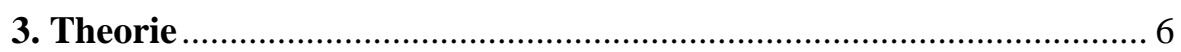

3.1 Ideale Radikalische Polymerisation ..................................................... 6

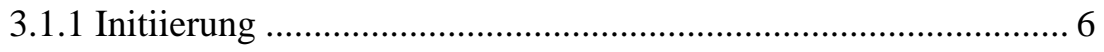

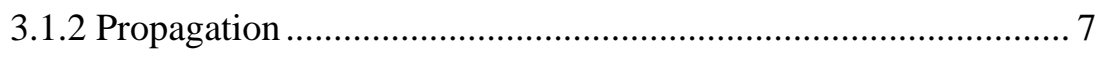

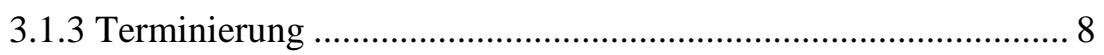

3.2 Quasistationäre Bedingungen ....................................................... 8

3.3 Umsatzabhängigkeit der Geschwindigkeitskoeffizienten.................... 9

3.3.1 Umsatzabhängigkeit der Propagation .......................................... 10

3.3.2 Umsatzabhängigkeit der Terminierung........................................ 11

3.4 Kettenlängenabhängigkeit der Geschwindigkeitskoeffizienten......... 14

3.4.1 Kettenlängenabhängigkeit der Propagation ................................ 14

3.4.2 Kettenlängenabhängigkeit der Terminierung ............................. 15

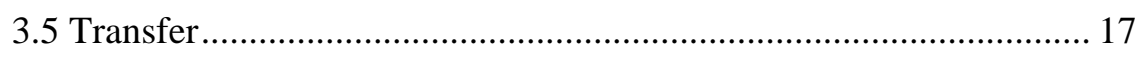

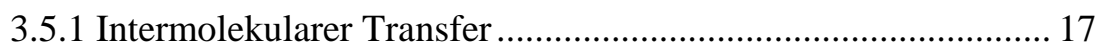

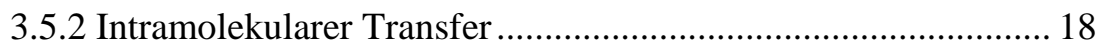

3.6 Propagationskoeffizienten mittels PLP-SEC ……………………..... 21 
3.7 Analyse der Kettenlängenabhängigkeit der Terminierung mittels SPPLP-ESR

4. Experimenteller Teil .................................................................. 26

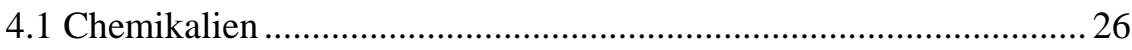

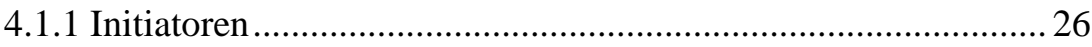

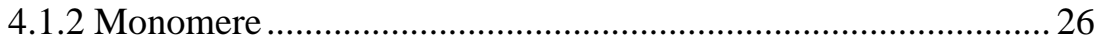

4.1.3 Weitere Chemikalien ................................................................ 27

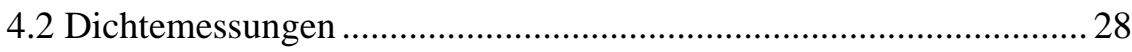

4.3 FT-NIR Spektroskopie .................................................................. 29

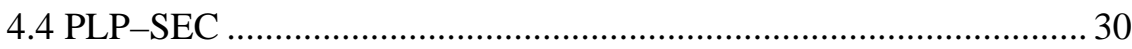

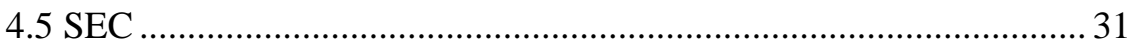

4.6 Elektronenspinresonanz ................................................................... 31

4.6.1 Experimenteller Aufbau............................................................. 31

4.6.2 Probenvorbereitung und Messdurchführung................................ 32

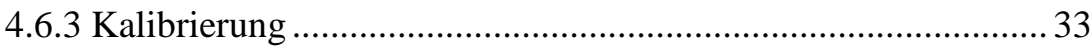

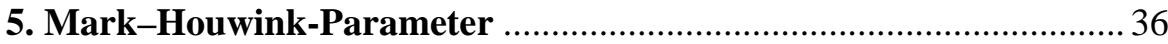

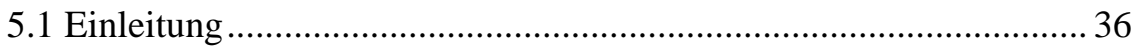

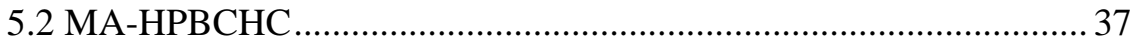

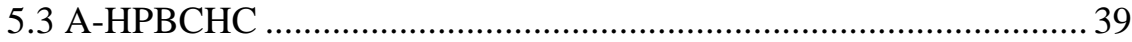

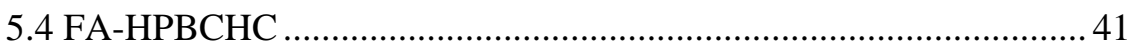

6. Propagation von MA-HPBCHC …………………............................. 49

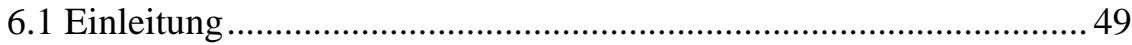

6.2 Strukturanalyse der PLP-SEC Kurven ............................................. 49

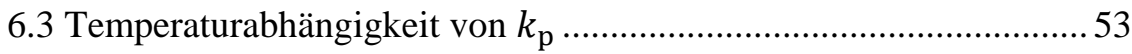

6.4 Propagation in nematischer Phase...................................................... 57

7. Propagation und backbiting von A-HPBCHC .................................... 60

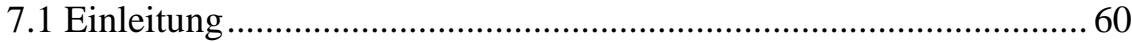

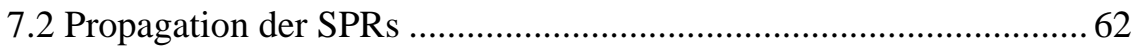


7.2.1 Strukturanalyse der PLP-SEC Kurven 62

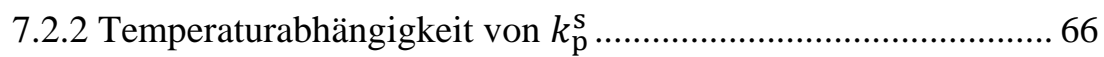

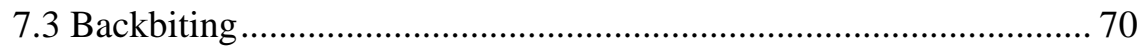

7.3.1 Das verwendete Modell ....................................................... 71

7.3.2 Die Temperaturabhängigkeit von $k_{\mathrm{bb}}$................................. 76

7.4 Zusammensetzung der Radikalpopulation................................... 78

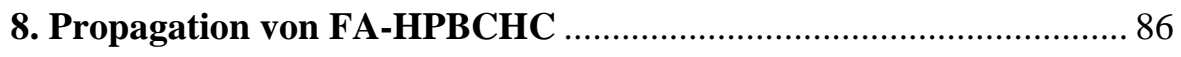

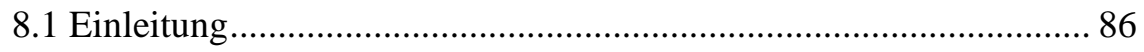

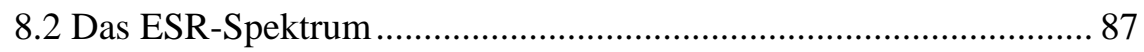

8.3 Strukturanalyse der PLP-SEC Kurven ....................................... 89

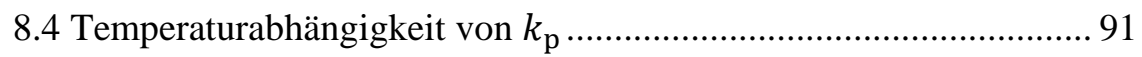

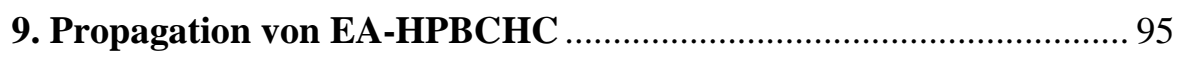

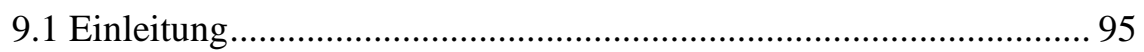

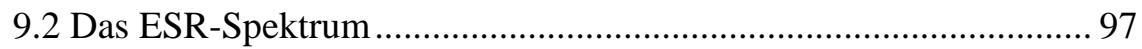

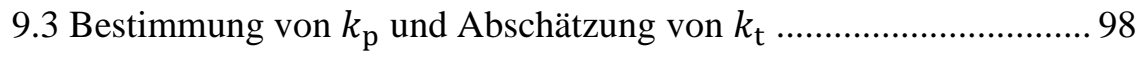

10. Terminierung von Methacrylaten in nematischer und chiralnematischer Phase ......................................................................... 102

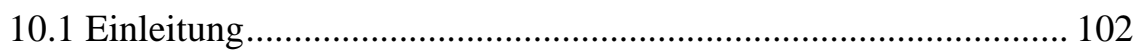

10.2 Das ESR-Spektrum von Methacrylaten in nematischer und chiralnematischer Phase..................................................................... 103

10.3 Ordnung der nematischen Phase im Magnetfeld des ESR-

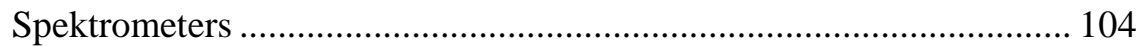

10.4 Terminierung von Methacrylaten in nematischer Phase.............. 107

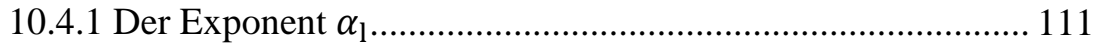

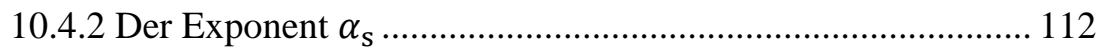

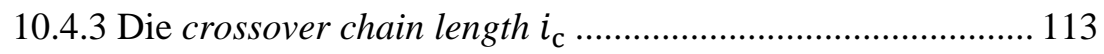

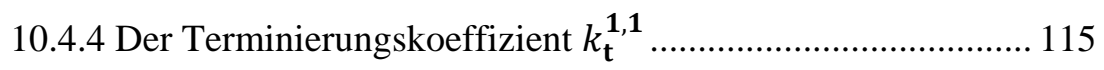

10.5 Terminierung von Methacrylaten in chiral-nematischer Phase .... 119 


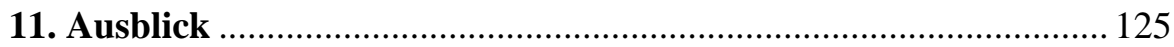

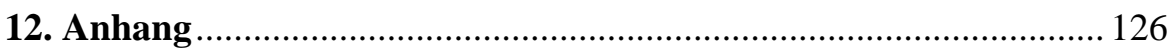

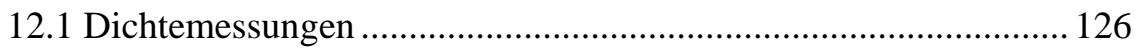

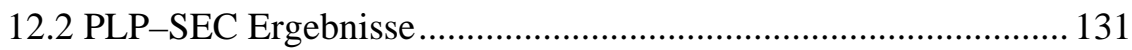

12.3 Abkürzüngsverzeichnis.................................................... 138

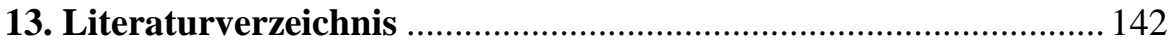

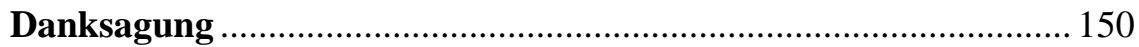

Biografie des Autors ................................................................... 151 


\section{Zusammenfassung}

Die vorliegende Arbeit befasst sich mit der Kinetik der radikalischen Polymerisation von reaktiven Mesogenen in isotroper und anisotroper Lösung. Kinetische Koeffizienten wurden mittels Pulslaser-induzierter Polymerisation in Verbindung mit Größenausschlusschromatographie (PLP-SEC), Einzelpuls Pulslaser-induzierter Polymerisation in Verbindung mit Elektronenspinresonanz-Spektroskopie (SP-PLP-ESR) und ESR mit kontinuierlicher Initiierung bestimmt. Das Programmpaket PREDICI ${ }^{\circledR}$ wurde zur Simulation der Prozesse verwendet.

Durch Reversible-Addition-Fragmentation-chain-Transfer- (RAFT-) Polymerisation wurden monodisperse Polymerstandards für ein Methacrylat (MA-HPBCHC), Acrylat (A-HPBCHC) und Fluoroacrylat (FA-HPBCHC) mit jeweils der mesogenen Seitengruppe Hexyl-4'-pentyl[1,1'-bi(cyclohexan)]-4-carboxylat (HPBCHC) hergestellt und durch SEC analysiert. Absolute Molmassen wurden für das Methacrylat und Acrylat durch Kombination von Lichtstreu- und Brechungsindexdetektion ermittelt. Für FA-HPBCHC wurden absolute Molmassen durch UV-Detektion der RAFT-Endgruppen bestimmt. Anschließend wurden durch Korrelation der absoluten und relativen Molmassen die Mark-Houwink-Koeffizienten der Polymere bestimmt. Deren Kenntnis erhöht maßgeblich die Genauigkeit der PLP-SEC Experimente.

Die Bestimmung der Propagationskoeffizienten von MA-HPBCHC und A-HPBCHC durch PLP-SEC ergab in beiden Fällen eine Verringerung von $k_{\mathrm{p}}$ entgegengesetzt zum literaturbekannten Trend für unverzweigte Alkylsubstituenten von höherer Propagationsgeschwindigkeit mit länger werdender Seitenkette. Diese Verringerung könnte durch einen entropisch 
weniger begünstigten Übergangzustand der Propagationsreaktion aufgrund der sterisch anspruchsvollen Seitengruppe zu erklären sein.

Durch Untersuchung der Pulsfrequenzabhängigkeit der PLP-SEC Ergebnisse von A-HPBCHC wurde durch Vergleich mit PREDICI ${ }^{\circledR}$ Simulationen der backbiting Koeffizient $k_{\mathrm{bb}}$ bestimmt. Hierbei zeigt sich im Vergleich zu Butylacrylat ein verstärktes Auftreten von backbiting. Dies äußert sich zusätzlich in den Anteilen an mid-chain Radikalen unter quasistationären Bedingungen, welche mittels ESR bestimmt wurden.

Die Propagationsgeschwindigkeit von FA-HPBCHC wurde mittels PLPSEC untersucht. Dabei handelt es sich um die erste Untersuchung von Fluoroacrylaten durch diese Methode. Als Resultat ergab sich ein hohes $k_{\mathrm{p}}$, welches ein Beleg für den starken Einfluss des Fluorsubstituenten auf die Propagationskinetik ist.

Die Analyse der Propagation des Ethacrylats EA-HPBCHC erfolgte mittels ESR-Spektroskopie in Verbindung mit Umsatzmessungen. EA-HPBCHC zeigte ein sehr niedriges $k_{\mathrm{p}}$ bei $60^{\circ} \mathrm{C}$. Aufgrund quasistationärer Zustände konnten zusätzlich Hinweise auf eine Kettenlängenabhängigkeit der Terminierung gefunden und die Parameter des Kurzkettenbereiches, $k_{\mathrm{t}}^{1,1}$ und $\alpha_{\mathrm{s}}$, abgeschätzt werden.

Die kettenlängenabhängige Terminierung von Ethylhexylmethacrylat (EHMA) und MA-HPBCHC wurde in der nematischen Flüssigkeit BL-087 mittels SP-PLP-ESR untersucht. Dabei zeigten die Makroradikale in flüssigkristalliner Lösung ein ähnliches Verhalten wie in isotroper Lösung. Das composite model lieferte eine akkurate Beschreibung der Radikalverläufe nach Initiierung durch einen Laserpuls. Die Exponenten $\alpha_{\mathrm{s}}$ und $\alpha_{1}$ ähneln denen anderer Methacrylate in Substanz. Die crossover chain length $\left(i_{\mathrm{c}}\right)$ hingegen ist für EHMA im Vergleich zu der Polymerisation in Substanz deutlich geringer. Die Auftragung von $k_{\mathrm{t}}^{1,1}$ nach Arrhenius lieferte einen linearen Zusammenhang, was gegen einen Einfluss des Ordnungsparameters auf $k_{\mathrm{t}}^{1,1}$ spricht. Die Zugabe von 5 bzw. $7 \mathrm{wt} \%$ des Dotierstoffes R-5011 zeigte keinen Einfluss auf $\alpha_{\mathrm{s}}, \alpha_{1}$ oder $i_{\mathrm{c}}$, beeinflusst jedoch $k_{\mathrm{t}}^{1,1}$. Mit steigendem Anteil an R-5011 erhöht sich die Aktivierungsenergie von $k_{\mathrm{t}}^{1,1}$. 


\section{Einleitung}

Polymere sind in der heutigen Zeit eine wichtige Materialgruppe zur Herstellung unterschiedlichster Produkte. Von der massenhaft hergestellten Plastiktüte aus Polyethylen bis hin zu Hochleistungsmaterialien, wie beispielsweise Polyetherketon in Flugzeugen, sind Polymere überall anzutreffen. ${ }^{[1]}$ Besonders das geringe Gewicht, die wirtschaftlichen Herstellungskosten und die hohe Korrosionsresistenz machen sie attraktiv.

Im industriellen Rahmen ist die konventionelle radikalische Polymerisation von großer Relevanz. Die Molmassenverteilung und somit die physikalischen Eigenschaften des Produktes dieser Herstellungsmethode ergeben sich aus der Kinetik der Reaktion. Dementsprechend einleuchtend ist der Antrieb ein vollständiges Verständnis davon zu erreichen. Durch Untersuchung der Teilschritte der Reaktion können Modelle entwickelt werden, die eine computergestützte Simulation der Reaktion unter unterschiedlichsten Reaktionsbedingungen erlauben. Dies resultiert in eine Kostenersparnis, da systematische Experimente unter Variation von Parametern wie Temperatur, Druck, Initiatorkonzentration, Monomerkonzentration, Reaktorgröße oder Reaktortyp überflüssig werden.

Das Verständnis von Propagation und Terminierung ist elementar zur Erstellung eines kinetischen Modells für die radikalische Polymerisation. Die Bestimmung der Koeffizienten $k_{\mathrm{p}}$ und $k_{\mathrm{t}}$ ist dabei unumgänglich. Methoden, die keine gekoppelten Parameter bestimmen, sind aufgrund der hohen Verlässlichkeit anzustreben. Zur Bestimmung von $k_{\mathrm{p}}$ eignet sich hervorragend die vom IUPAC-Komitee „Modeling of Polymerization Kinetics and Processes“ empfohlene pulslaserinduzierte-Polymerisation in 
Verbindung mit Größenausschlusschromatographie (PLP-SEC). ${ }^{[2,3]}$ Diese Methode ist ausführlich validiert worden und kam vielfach für Monomere in organischer und wässriger Lösung zur Anwendung. ${ }^{[4-11]}$ Zur erfolgreichen Bestimmung von $k_{\mathrm{p}}$ mittels PLP-SEC sind einige Randbedingungen und Grundvoraussetzungen zu beachten. ${ }^{[12]}$

Die Elektronspinresonanz-Spektroskopie (ESR-Spektroskopie) erlaubt die direkte Messung der Radikalkonzentration. Wird diese nach Eintreffen eines Laserpulses verfolgt, kann der Terminierungsvorgang inklusive der auftretenden Kettenlängenabhängigkeit ausführlich untersucht werden. Das prägnante Akronym SP-PLP-ESR beschreibt diese Einzelpuls-PulslaserPolymerisation in Verbindung mit ESR-Spektroskopie. ${ }^{[13,14]}$ Nicht nur quantitative auch qualitative Informationen können mittels ESR gewonnen werden. Die Struktur und die Einschränkung der Rotationsfreiheit entlang des Polymerrückgrats von propagierenden Radikalen ist ausführlich untersucht worden. ${ }^{[15,16]}$ ESR-Spektroskopie erlaubt zusätzlich die Identifizierung unterschiedlicher Radikalspezies, welches aufschlussreiche Erkenntnisse zu Transferreaktion wie backbiting zutage brachte. ${ }^{[17-19]}$

Eine außergewöhnliche Anwendung der radikalischen Polymerisation liegt in der Herstellung von speziellen Flüssigkristallanzeigen (Liquid crystal display, LCD). Dabei kann durch Polymerisation in einer flüssigkristallinen Phase die Temperaturstabilität und die elektrooptischen Eigenschaften dieser Mischung stark beeinflusst werden. ${ }^{[20]}$ Solche Materialien werden Polymer-stabilized liquid crystals genannt. Hierbei verwendet typischerwiese Monomeranteile von bis zu $10 \mathrm{wt} \%$. Prominente Beispiele sind Polymer-stabilized vertical alignment cells und Polymer-stabilized liquid crystal blue phases. ${ }^{[21-23]}$ Häufig werden für solche Anwendungen Monomere verwendet die anisotrope Eigenschaften aufweisen, oftmals eingeführt in der Seitengruppe, die so genannten reaktiven Mesogene. Die Kinetik der Polymerisation kontrolliert, auch in diesen Systemen, die Molmassenverteilung des Polymerproduktes und somit schlussendlich die Eigenschaften des Polymers. Die Eigenschaften des Polymers haben Einfluss auf das Maß der Interaktion zwischen Polymer und flüssigkristalliner Phase. Diese Interaktion beeinflusst die elektrooptischen Eigenschaften der Mischung. Dies führt somit letztendlich zu einer Korrelation zwischen Polymerisationskinetik und elektrooptischen Eigenschaften der Mischung. Diese Korrelation wurde von Iwata et al. für 
Polymer-stabilized liquid crystal blue phases beobachtet, indem die Reaktionsrate mit der resultierenden Temperaturstabilität der Phasen verglichen wurde. ${ }^{[24]}$

Die Kinetik der radikalischen Polymerisation in flüssigkristalliner Phase ist dementsprechend von großem Interesse und bietet die Möglichkeit der Optimierung von elektrooptischen Eigenschaften durch gezieltere Auswahl von Reaktionsbedingungen zur Erstellung von leistungsoptimierten Polymerprodukten. Während über die Messung von Reaktionsraten in flüssigkristallinen Medium in der Vergangenheit berichtet worden ist, sind dem Autor bis zum heutigen Zeitpunkt keine Studien bekannt in denen einzelne Reaktionsschritte mittels PLP-SEC oder SP-PLP-ESR untersucht worden sind. ${ }^{[25-27]}$ Somit blieben grundlegende Fragen zur radikalischen Polymerisation in Flüssigkristallen ungelöst. Beispielsweise welchen Einfluss mesogene Seitengruppen auf die Propagationsgeschwindigkeit der einzelnen Monomerklassen wie Methacrylaten oder Acrylaten haben. Oder in wieweit backbiting auch mesogene Acrylate betrifft. Von Interesse ist zudem der Einfluss der flüssigkristallinen Phasen auf die Terminierungsreaktion.

Ziel dieser Arbeit ist es einen Beitrag zum Verständnis der Kinetik der radikalischen Polymerisation in Flüssigkristallen zu leisten. Dazu wird die Bestimmung der Propagationskoeffizienten von jeweils einem Methacrylat, Acrylat, Fluoroacrylat und Ethacrylat mit der gleichen flüssigkristallinen Seitengruppe (Hexyl-4'-pentyl-[1,1'-bi(cyclohexan)]-4-carboxylat, HPBCHC) vorgestellt. Dies wurde in den ersten drei Fällen mittels PLPSEC bewerkstelligt und im letzteren mittels ESR-Spektroskopie in Kombination mit Umsatzmessungen. Als Voraussetzung für die PLP-SEC Untersuchungen wird die Bestimmung der Mark-Houwink-Koeffizienten der Monomere durch SEC mit gekoppelter Mehrfachdetektion präsentiert.

Weiterhin wird die Terminierung des mesogenen Methacrylats 6(Methacryloyloxy)-hexyl-4'-pentyl-[1,1'-bi(cyclohexan)]-4-carboxylat (MA-HPBCHC) und des isotropen Monomers Ethylhexylmethacrylats beschrieben. Dabei wird die Kettenlängenabhängigkeit mittels SP-PLPESR vollständig aufgeschlüsselt und zusätzlich der Effekt der Zugabe eines chiralen Dotierstoffes (R-5011, Merck KGaA) vorgestellt. 


\section{Theorie}

\subsection{Ideale Radikalische Polymerisation}

\subsubsection{Initiierung}

Die Initiierung der radikalischen Polymerisation erfolgt durch den Zerfall eines Initiatorsmoleküls in Primärradikale und kann photochemisch, thermisch oder durch Redoxprozesse verursacht werden. Die entstandenen Radikale reagieren im darauffolgenden Schritt mit dem Monomer und führen somit zum Kettenstart.

$$
\begin{gathered}
\mathrm{I} \stackrel{k_{\mathrm{d}} \cdot f}{\longrightarrow} 2 \mathrm{R}_{0}^{\bullet} \\
\mathrm{R}_{0}^{\bullet}+\mathrm{M} \stackrel{k_{\mathrm{i}}}{\longrightarrow} \mathrm{R}_{1}^{\bullet}
\end{gathered}
$$

Hierbei soll I für den Initiator, $\mathrm{R}_{0}^{\bullet}$ für ein Initiatorfragmentradikal, M für das Monomer und $\mathrm{R}_{1}^{\bullet}$ für ein Radikal der Kettenlänge eins stehen. $k_{d}$ bezeichnet den Geschwindigkeitskoeffizienten des Initiatorzerfalls und $k_{\mathrm{i}}$ den Geschwindigkeitskoeffizienten der Addition eines Monomermoleküls an das Initiatorfragmentradikal. Die Initiatoreffizienz $f$ bezeichnet den Anteil an Primärradikalen die, ohne zu rekombinieren, den Lösungsmittelkäfig verlassen und Kettenwachstum initiieren.

Der homolytische thermische Zerfall eines Initiators kann durch ein Geschwindigkeitsgesetz erster Ordnung beschrieben werden. 


$$
\frac{d c_{\mathrm{R}}}{d t}=2 \cdot k_{\mathrm{d}} \cdot f \cdot c_{\mathrm{I}}
$$

Im Falle einer photochemischen Initiierung durch einen Laserpuls lässt sich die Konzentration der entstehenden Radikale durch (3.2) beschreiben. Aufgrund der kurzen Pulsbreiten (ca. 20 ns), ist die Entstehung der Radikale instantan im Vergleich zu den restlichen Reaktionsschritten.

$$
c_{\mathrm{R}}^{0}=2 \cdot \Phi \cdot \frac{n_{\mathrm{abs}}}{V}
$$

Hierbei kennzeichnet $\Phi$ die Primärquantenausbeute, $n_{\text {abs }}$ die Anzahl an absorbierten Photonen und $V$ das entsprechende Volumen. Die Primärquantenausbeute ist das Produkt der Initiatoreffizienz und dem Anteil der absorbierten Protonen, die zu einem Initiatorzerfall geführt haben.

\subsubsection{Propagation}

Der Begriff Propagation beschreibt das Kettenwachstum durch Addition von Monomer an ein Radikal der Kettenlänge $i, \mathrm{R}_{i}$. Hierbei entsteht ein Makroradikal mit der Kettenlänge $i+1, \mathrm{R}_{i+1}^{\bullet} \cdot k_{\mathrm{p}}$ entspricht hierbei dem Geschwindigkeitskoeffizienten der Reaktion.

$$
\mathrm{R}_{i}^{\bullet}+\mathrm{M} \stackrel{k_{\mathrm{p}}}{\longrightarrow} \mathrm{R}_{i+1}^{\bullet}
$$

Die Änderung der Monomerkonzentration lässt sich durch das Geschwindigkeitsgesetz (3.3) beschreiben, mit dem Geschwindigkeitskoeffizienten $k_{\mathrm{p}}$.

$$
\frac{\mathrm{dc}_{\mathrm{M}}}{\mathrm{d} t}=-k_{\mathrm{p}} \cdot c_{\mathrm{M}} \cdot c_{\mathrm{R}}
$$




\subsubsection{Terminierung}

Terminierung bezeichnet die Reaktion zweier Radikale miteinander und erfolgt entweder durch Kombination oder Disproportionierung. Im ersten Fall bedeutet dies eine Kombination der zwei Radikale mit dem Geschwindigkeitskoeffizienten $\quad k_{\mathrm{t}, \mathrm{c}} \quad \mathrm{zu}$ einem desaktivierten Polymermolekül $P_{i+j}$, dessen Kettenlänge der Summe der Kettenlängen beider Makroradikale entspricht. Der zweite Fall beschreibt die Übertragung eines $\quad \beta$-Wasserstoffatoms mit dem Geschwindigkeitskoeffizienten $k_{\mathrm{t}, \mathrm{d}}$, wobei zwei desaktivierte Polymermoleküle entstehen $\left(P_{i}\right.$ und $\left.P_{j}\right)$.

$$
\begin{gathered}
\mathrm{R}_{\mathrm{i}}^{\cdot}+\mathrm{R}_{\mathrm{j}}^{\cdot} \stackrel{k_{\mathrm{t}, \mathrm{c}}}{\longrightarrow} \mathrm{P}_{\mathrm{i}+\mathrm{j}} \\
\mathrm{R}_{\mathrm{i}}^{\bullet}+\mathrm{R}_{\mathrm{j}}^{\cdot} \stackrel{k_{\mathrm{t}, \mathrm{d}}}{\longrightarrow} \mathrm{P}_{\mathrm{i}}+\mathrm{P}_{\mathrm{j}}
\end{gathered}
$$

Das Geschwindigkeitsgesetz der Terminierungsreaktion ist zweiter Ordnung und lässt sich als Abnahme der Radikalkonzentration angeben. Der Geschwindigkeitskoeffizient der Terminierung $k_{\mathrm{t}}$ entspricht der Summe von $k_{\mathrm{t}, \mathrm{c}}$ und $k_{\mathrm{t}, \mathrm{d}}$.

$$
\frac{\mathrm{d} c_{\mathrm{R}}}{\mathrm{d} t}=-2 \cdot k_{\mathrm{t}} \cdot c_{\mathrm{R}}^{2}
$$

Im Rahmen dieser Arbeit wird die von IUPAC empfohlene Konvention der Inklusion des Faktors 2 in das Geschwindigkeitsgesetz angewendet. ${ }^{[28]}$

\subsection{Quasistationäre Bedingungen}

Wird ein stationärer Zustand angenommen, bleibt die Radikalkonzentration im Laufe der Reaktion konstant. Dies bedeutet die Radikalbildungsrate muss der Terminierungsrate entsprechen. Dementsprechend ergibt sich durch Kombination von (3.1) und (3.4) 


$$
k_{\mathrm{d}} \cdot f \cdot c_{\mathrm{I}}=k_{\mathrm{t}} \cdot c_{\mathrm{R}}^{2}
$$

Durch Einsetzen von (3.5) in (3.3) lässt sich die Polymerisationsgeschwindigkeit $R_{\mathrm{p}}$ als zeitliche Abnahme der Monomerkonzentration in Abhängigkeit der Geschwindigkeitskoeffizienten von Initiatorzerfall, Propagation und Terminierung darstellen.

$$
R_{\mathrm{p}}=-\frac{\mathrm{d} c_{\mathrm{M}}}{\mathrm{d} t}=\frac{k_{\mathrm{p}}}{\sqrt{k_{\mathrm{t}}}} \cdot \sqrt{k_{\mathrm{d}} \cdot f \cdot c_{\mathrm{I}}} \cdot c_{\mathrm{M}}
$$

\subsection{Umsatzabhängigkeit der Geschwindigkeitskoeffizienten}

Diffusion ist notwendigerweise ein Teilschritt der bimolekularen Reaktionschritte in radikalischen Polymerisationen. Im Verlauf einer Polymerisation kommt es aufgrund der Löslichkeitseigenschaften des Polymerprodukts bei steigenden Umsätzen zu einer Veränderung der Viskosität der Lösung. Nach der Einstein-Stokes-Beziehung gibt es eine Relation zwischen dem Diffusionskoeffizienten $D$ und der dynamischen Viskosität $\eta$ der Lösung.

$$
D^{i}=\frac{k_{\mathrm{B}} \cdot T}{6 \cdot \pi \cdot r_{i} \cdot \eta}
$$

Dabei bezeichnet $k_{\mathrm{B}}$ die Bolzmannkonstante, $T$ die Temperatur und $r_{i}$ den hydrodynamischen Radius des Makromoleküls. Diese Abhängigkeit resultiert in einer Umsatzabhängigkeit der Reaktionsschritte in der radikalischen Polymerisation. 


\subsubsection{Umsatzabhängigkeit der Propagation}

Die Propagation kann in zwei konsekutive Teilschritte unterteilt werden: Die Diffusion des Makromoleküls und Monomers aufeinander zu und die chemische Reaktion. Dementsprechend ergibt sich für $k_{\mathrm{p}}$ folgende Abhängigkeit. ${ }^{[29]}$

$$
\frac{1}{k_{\mathrm{p}}}=\frac{1}{k_{\mathrm{p}, 0}}+\frac{1}{k_{\mathrm{p}, \mathrm{D}}}
$$

Hierbei beschreibt $k_{\mathrm{p}, 0}$ den Propagationsschritt ohne Diffusionsbeitrag und $k_{\mathrm{p}, \mathrm{D}}$ den Diffusionsprozess der Propagationsreaktion. Der Geschwindigkeitskoeffizient $k_{\mathrm{D}}$ einer diffusionskontrollierten Reaktion kann durch die Smoluchowski-Gleichung beschrieben werden: ${ }^{[30]}$

$$
k_{\mathrm{D}}=4 \cdot \pi \cdot N_{\mathrm{A}} \cdot\left(D_{\mathrm{S}}^{\mathrm{A}}+D_{\mathrm{S}}^{\mathrm{B}}\right) \cdot R_{\mathrm{c}}
$$

Hierbei kennzeichnen $D_{\mathrm{S}}^{\mathrm{A}}$ und $D_{\mathrm{S}}^{\mathrm{B}}$ die Diffusionskoeffizienten der Reaktanden und $R_{\mathrm{c}}$ den Einfangradius. Aufgrund der sehr viel kleineren Molekularradien wird der Diffusionsschritt der Propagation vorwiegend von der Monomerdiffusion bestimmt.

Aus dem vorrangegangenen lässt sich nun für $k_{\mathrm{p}, \mathrm{D}}$ eine umgekehrt proportionale Abhängigkeit zur umsatzabhängigen relativen Viskosität der Lösung $\eta_{\mathrm{r}}(X)$ annehmen.

$$
k_{\mathrm{p}, \mathrm{D}}=\frac{k_{\mathrm{p}, \mathrm{D}}^{0}}{\eta_{\mathrm{r}}(X)}
$$

Hier ist $k_{\mathrm{p}, \mathrm{D}}^{0}$ der Geschwindigkeitskoeffizient des Diffusionsprozesses der Propagationsreaktion bei $X=0$. Dementsprechend ist die Propagationsrate eine Funktion der Viskosität, die sich bei einer linearen Polymerisation gewöhnlicherweise erst bei hohen Umsätzen von über 0,8 stark verändert. 
Somit ist $k_{\mathrm{p}}$ in einem weiten Umsatzbereich chemisch kontrolliert und nahezu konstant. Erreicht die Reaktion jedoch hohe Umsätze und eine daraus erfolgende hohe Viskosität, tritt Diffusionskontrolle ein. Dieser Übergangspunkt wird als Glaspunkt bezeichnet. Die Diffusionskontrolle sorgt ab diesem Punkt für eine Verringerung von $k_{\mathrm{p}}$, wie ausgiebig für Styrol und MMA gezeigt wurde. ${ }^{[31,32]}$

\subsubsection{Umsatzabhängigkeit der Terminierung}

Die Terminierungsreaktion kann wie im Falle der Propagation durch Diffusion und anschließender Reaktion der zwei Radikale beschrieben werden. Hierbei wird aufgrund der hohen Reaktivität der Radikale eine Diffusionskontrolle angenommen. ${ }^{[33]}$ Diese Annahme wird durch die Tatsache gestützt, dass für $k_{\mathrm{t}}$ eine umgekehrt proportionale Abhängigkeit von $\eta$ beobachtet werden kann und dass $k_{\mathrm{t}}$ mit zunehmendem Druck abnimmt. $^{[34,35]}$

Der Mechanismus der Terminierungsreaktion wurde von Benson und North als dreischrittiger Prozess beschrieben. ${ }^{[36]}$ Dabei finden konsekutiv Translationsdiffusion, Segmentdiffusion und zuletzt die chemische Reaktion statt(siehe Abbildung 3.1). Die Translationsdiffusion kennzeichnet die massenzentrierte Diffusion zweier Makroradikale zueinander und die Segmentdiffusion die Neuorientierung der geknäulten Polymerketten die eine Annäherung der reaktiven Radikalzentren und somit die chemische Reaktion erlaubt.

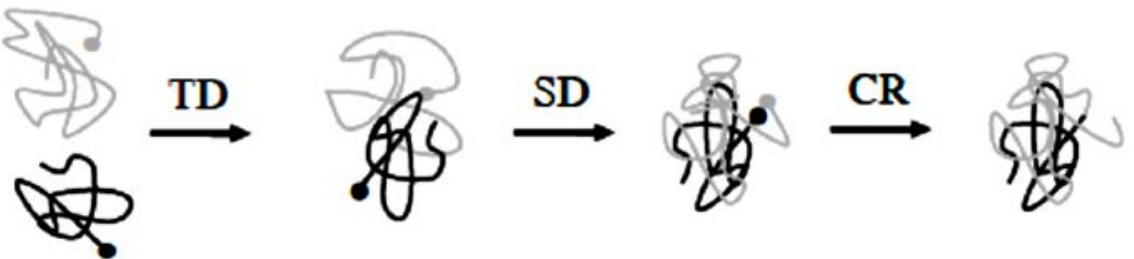

Abbildung 3.1: Darstellung der Terminierungsreaktion unterteilt in Reaktionsschritten: Translationsdiffusion (TD), Segmentdiffusion (SD) und chemische Reaktion (CR). ${ }^{[37]}$

Der Geschwindigkeitskoeffizient $k_{\mathrm{t}}$ zeigt nach diesem Modell folgende Abhängigkeit: ${ }^{[29]}$ 


$$
\frac{1}{k_{\mathrm{t}}}=\frac{1}{k_{\mathrm{TD}}}+\frac{1}{k_{\mathrm{SD}}}+\frac{1}{k_{\mathrm{CR}}} .
$$

Die Translationsdiffusionsrate lässt sich ins Verhältnis zur umsatzabhängigen relativen Viskosität des Reaktionsmediums setzen.

$$
k_{\mathrm{TD}}=\frac{k_{\mathrm{TD}}^{0}}{\eta_{\mathrm{r}}(X)}
$$

Hierbei kennzeichnet $k_{\text {TD }}^{0}$ den Translationsdiffusionskoeffizienten bei einem Umsatz von null. Dieses Modell ist ausreichend zur Beschreibung von realen Systemen solange kein hoher Umsatz erreicht wird. Erreicht das System einen hohen Umsatz und eine dementsprechend hohe Viskosität, kommt es zu einer starken Hinderung der Diffusionsprozesse. Ab diesem Zeitpunkt muss die Reaktionsdiffusion beachtet werden. Hierbei findet die Annäherung der reaktiven Radikalzentren nicht durch Diffusion statt, stattdessen wird der Abstand der Reaktionszentren durch Propagation überwunden. Dementsprechend ist der Reaktionsdiffusionskoeffizient $k_{\mathrm{RD}}$ proportional zu $k_{\mathrm{p}}$ und zur Reaktionsdiffusionskonstante CRD.

$$
k_{\mathrm{RD}}=C_{\mathrm{RD}} \cdot k_{\mathrm{p}} \cdot \frac{c_{\mathrm{M}}}{c_{\mathrm{M}}^{0}}=C_{\mathrm{RD}} \cdot k_{\mathrm{p}} \cdot(1-X)
$$

Aus diesem Zusammenhang kann die Beschreibung von $k_{\mathrm{t}}$ ergänzt werden.

$$
k_{\mathrm{t}}=\frac{1}{\frac{1}{k_{\mathrm{TD}}}+\frac{1}{k_{\mathrm{SD}}}+\frac{1}{k_{\mathrm{CR}}}}+k_{\mathrm{RD}}
$$

Aus (3.10), (3.12), (3.13) und (3.14) ergibt sich: 


$$
k_{\mathrm{t}}=\frac{1}{\frac{\eta_{r}(X)}{k_{\mathrm{TD}}^{0}}+\frac{1}{k_{\mathrm{SD}}}}+\frac{C_{\mathrm{RD}} \cdot(1-X)}{\frac{1}{k_{\mathrm{p}}}+\frac{\eta_{r}(X)}{k_{\mathrm{p}, \mathrm{D}}^{0}}}
$$

Diese Gleichung liefert eine angemessene Beschreibung von $k_{\mathrm{t}}$ mit fortlaufendem Umsatz für die bis zum jetzigen Zeitpunkt untersuchten Systeme.

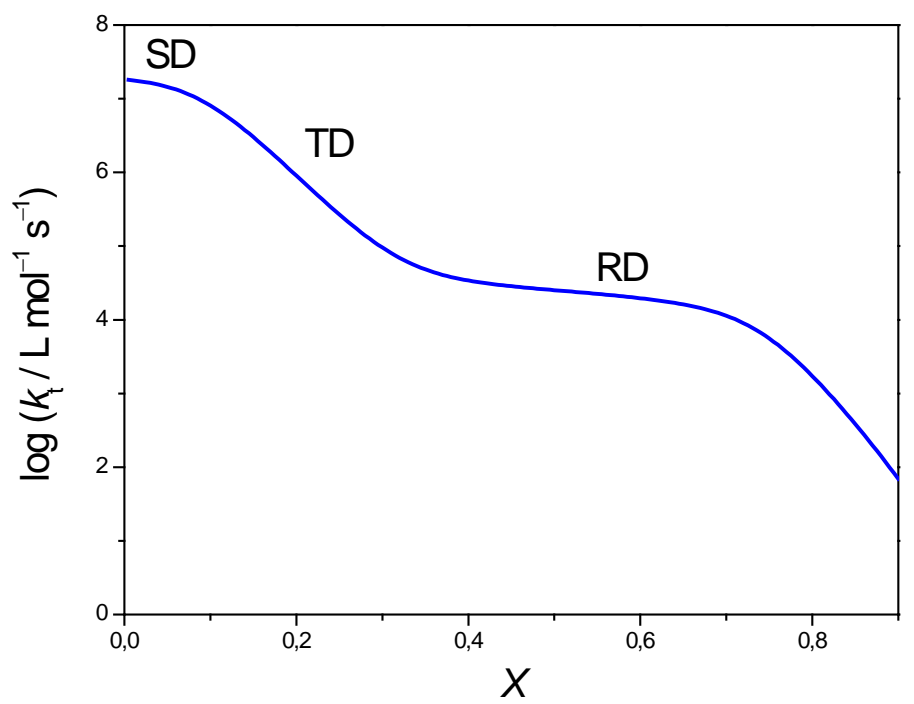

Abbildung 3.2: Schematische Darstellung der Abhängigkeit des Terminierungskoeffizienten von dem Umsatz. Der kontrollierende Schritt im jeweiligen Segment ist als SD, TD und RD gekennzeichnet, analog zu der Beschreibung im Text.

Wird nun der Verlauf von $k_{\mathrm{t}}$ in Bezug auf den Monomerumsatz für ein gegebenes System betrachtet, welcher schematisch in Abbildung 3.2 dargestellt ist, kann dieser in drei Bereiche unterteilt werden. Bei niedrigem Umsatz ist nur eine geringfügige Änderung von $k_{\mathrm{t}} \mathrm{zu}$ beobachten, welche für eine Kontrolle durch Segmentdiffusion als geschwindigkeitsbestimmenden Schritt spricht, da diese langsamer verläuft als die Translationsdiffusion. Steigt der Umsatz an kommt es zu einer Viskositätsänderung aufgrund der gelösten Polymerketten. Dies hat eine 
Hinderung der Translationsdiffusion und demensprechend eine Kontrolle durch $k_{\mathrm{TD}}$ zur Folge. Die Verringerung von $k_{\mathrm{t}}$ äußert sich in einer Erhöhung der Polymerisationsrate, was gewöhnlicherweise als Gel-Effekt oder Trommsdorff-Norrish-Smith-Effekt bezeichnet wird. ${ }^{[38]}$ Bei hohem Umsatz sind die Diffusionsprozesse derart verlangsamt dass Reaktionsdiffusion als geschwindigkeitsbestimmender Schritt einsetzt. Somit sinkt die Terminierungsrate mit steigenden Umsatz aufgrund der Proportionalität von $k_{\mathrm{RD}}$ und $k_{\mathrm{p}}$.

\subsection{Kettenlängenabhängigkeit der Geschwindigkeitskoeffizienten}

Die Berücksichtigung der Kettenlängenabhängigkeit der Geschwindigkeitskoeffizienten ist unabdingbar für ein vollständiges Verständnis der ablaufenden Prozesse in einer radikalischen Polymerisation. Aufgrund der Diffusionskontrolle der Terminierungsreaktion ist die Kettenlängenabhängigkeit in diesem Falle viel stärker ausgeprägt als im Falle der Propagation, die vorwiegend chemisch kontrolliert ist.

\subsubsection{Kettenlängenabhängigkeit der Propagation}

Gewöhnlicherweise wird die Propagation in guter Näherung als kettenlängenunabhängig betrachtet. Experimentelle Befunde, die mittels PLP-SEC und PLP-MALDI-ToF-MS (Matrix-Assisted-Laser-DesorptionIonization Time-of-Flight Mass-Spectrometry) gewonnen werden konnten, legen nahe, dass im Oligomeren Bereich (Kettenlängen bis 10) ein Einfluss von der Kettenlänge auf $k_{\mathrm{p}}$ vorliegt, der den Koeffizienten bis zu einer Größenordnung weit erhöhen kann. ${ }^{[39,40]}$ Diese Abhängigkeit kann folgendermaßen beschrieben werden: ${ }^{[41]}$

$$
k_{\mathrm{p}}^{i}=k_{\mathrm{p}}\left[1+C_{1} \cdot \exp \left(-\frac{\ln 2}{i_{1 / 2}} \cdot(i-1)\right)\right]
$$


Hierbei beschreibt $i_{1 / 2}$ eine Halbwertszeit-Kettenlänge die als charakteristische Kettenlänge verstanden werden kann, bis hin zu der der Einfluss der Kettenlängenabhängigkeit auf $k_{\mathrm{p}}$ vorherrscht. $C_{1}$ ist ein Proportionalitätsfaktor der die Verringerung von $k_{\mathrm{p}}$ beschreibt.

$$
C_{1}=\frac{k_{\mathrm{p}}^{1}-k_{\mathrm{p}}}{k_{\mathrm{p}}}
$$

\subsubsection{Kettenlängenabhängigkeit der Terminierung}

Die starke Ausprägung der Kettenlängenabhängigkeit der Terminierung erscheint offensichtlich unter Berücksichtigung der Diffusionskontrolle dieses Reaktionsschrittes. Durch Addition von Monomereinheiten kommt es zur Änderung des hydrodynamischen Radius und dementsprechend nach (3.7) zu einer Änderung des Diffusionskoeffizienten. Dementsprechend muss zur Beschreibung der Terminierung ein Koeffizient verwendet werden, der die Kettenlängen $i$ und $j$ der zwei beteiligten Makroradikale berücksichtigt, $k_{\mathrm{t}}(i, j)$. In Pulslaser-induzierten Experimenten wie PLPSEC und SP-PLP-ESR kann aufgrund der zeitgleichen Initiierung aller Ketten davon ausgegangen werden, dass $i=j$ gilt. In diesem Falle eignet sich das composite model von Smith, Russel und Heuts zur Darstellung des Terminierungsprozesses. $^{[42]}$ Hierbei wird $k_{\mathrm{t}}$ in zwei Einflussbereiche unterteilt, den Kurzkettenbereich und den Langkettenbereich. Der Übergang wird durch eine kritische Kettenlänge, die so gennante crossover chain length $i_{\mathrm{c}}$ definiert. Somit ergeben sich für die einzelnen Bereiche folgende Abhängigkeiten von $k_{\mathrm{t}}(i, i)$ :

$$
k_{\mathrm{t}}(i, i)=k_{\mathrm{t}}(1,1) \cdot i^{-\alpha_{\mathrm{s}}} ; i \leq i_{c}
$$




$$
k_{\mathrm{t}}(i, i)=k_{\mathrm{t}}(1,1) \cdot i_{\mathrm{c}}^{-\alpha_{\mathrm{s}}+\alpha_{1}} \cdot i^{-\alpha_{1}}=k_{\mathrm{t}}^{0} \cdot i^{-\alpha_{1}} ; i>i_{\mathrm{c}}
$$

$k_{\mathrm{t}}(1,1)$ bezeichnet den Koeffizienten für die Terminierung zweier Radikale der Kettenlänge eins. Zu erwarten ist bezüglich dieses Wertes eine direkte Relation zu (3.9). $k_{\mathrm{t}}^{0}$ beschreibt das hypothetische Verhalten zweier geknäuelter Makroradikale der Kettenlänge eins, während $\alpha_{\mathrm{s}}$ und $\alpha_{1}$ das Ausmaß der Kettenlängenabhängigkeit für den Kurz- und Langkettenbereich angeben. Die meisten Untersuchungen ergaben keine Abhängigkeit von $\alpha_{\mathrm{s}}$, $\alpha_{1}$ oder $i_{\mathrm{c}}$ von der Temperatur. ${ }^{[34,43,44]}$ Sörensen hingegen fand für Ethylhexylmethacrylat (EHMA) eine starke Verringerung von $i_{\mathrm{c}}$ mit der Temperatur, die er mit der starken Hinderung der internen Rotation aufgrund der $\alpha$-Methylgruppe begründete. ${ }^{[4]}$ Die Abhängigkeit von $k_{\mathrm{t}}(1,1)$ von der Kettenlänge nach dem hier vorgestellten composite model ist in Abbildung 3.3 zur Übersicht graphisch dargestellt.

Wie oben erwähnt kann ein Bezug zwischen $k_{t}(1,1)$ und der Smoluchowski-Gleichung aufgestellt werden. Da es sich bei der Terminierung um eine Reaktion zweier Radikale handelt die nur im Falle von Singulett-Spinmultiplizität reagieren können, muss der rechte Term von (3.9) aus statistischen Gründen mit 0,25 multipliziert werden. ${ }^{[46]}$ Wird zusätzlich angenommen, dass sich die Diffusionskoeffizienten mittels Einstein-Stokes (3.7) beschreiben lassen und dass $D_{1}^{\mathrm{A}}=D_{1}^{\mathrm{B}}$, ergibt sich:

$$
k_{t}(1,1)=\frac{R \cdot T}{6 \cdot \eta} \cdot \frac{R_{\mathrm{c}}}{r_{i}}
$$

Für sphärisch geformte Radikale ergibt sich der Einfangradius als Summe der hydrodynamischen Radien $R_{\mathrm{c}}=2 r_{i}$. Daraus ergibt sich das sogenannte Diffusionslimit für $k_{t}(1,1)$, welches die maximale theoretische Terminierungsrate für eine gegebene Temperatur und Viskosität angibt. 


$$
k_{t}(1,1)=\frac{R \cdot T}{3 \cdot \eta}
$$

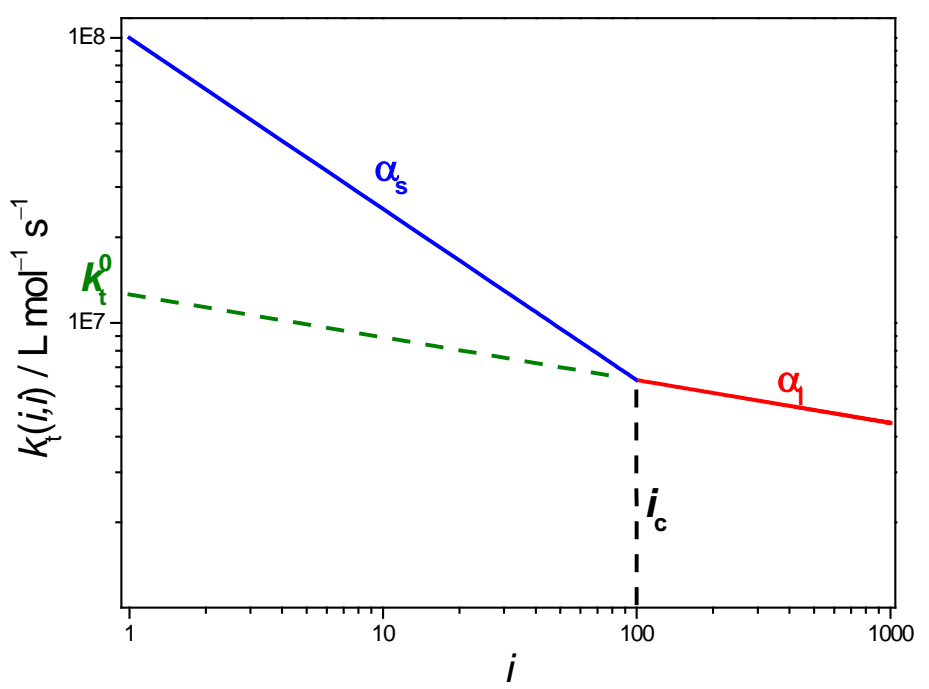

Abbildung 3.3: Schematische Darstellung der Abhängigkeit von $k_{t}(1,1)$ von der Kettenlänge $i$ nach dem composite model. Die Parameter des Modellsystems betragen $k_{t}(1,1)=10^{8} \mathrm{~L} \mathrm{~mol}^{-1} \mathrm{~s}^{1}, \alpha_{s}=0,6, \alpha_{l}=0,15$ und $i_{c}=100$.

Aus (3.21) ergibt sich dementsprechend eine grundlegende Beziehung zwischen $k_{t}(1,1)$ und der Fluidität der Lösung. Daraus ergibt sich die Erwartung einer ähnlichen Aktivierungsenergie von $k_{t}(1,1)$ und $\eta^{-1}$.

\subsection{Transfer}

\subsubsection{Intermolekularer Transfer}

Intermolekularer Transfer bezeichnet bei radikalischen Polymerisationen die Übertragung der Radikalfunktionalität auf ein anderes Molekül wie z. B. Lösungsmittel, Monomer, Polymer oder Transferagens. Die Übertragung erfolgt mit dem Geschwindigkeitskoeffizienten $k_{\mathrm{tr}, \mathrm{X}}$. Das neugebildete 
Radikal ist in der Lage eine Monomereinheit mit dem Geschwindigkeitskoeffizienten $k_{\mathrm{p}, \mathrm{X}} \mathrm{zu}$ addieren.

$$
\begin{aligned}
& \mathrm{R}_{i}^{\bullet}+\mathrm{X} \stackrel{k_{\mathrm{tr}, \mathrm{X}}}{\longrightarrow} \mathrm{P}_{i}+\mathrm{X}^{\bullet} \\
& \mathrm{X}^{\bullet}+\mathrm{M} \stackrel{k_{\mathrm{p}, \mathrm{X}}}{\longrightarrow} \mathrm{X}-\mathrm{M}^{\bullet}
\end{aligned}
$$

Das Geschwindigkeitsgesetz ist somit zweiter Ordnung:

$$
-\frac{\mathrm{d} c_{\mathrm{X}}}{\mathrm{d} t}=k_{\mathrm{tr}, \mathrm{X}} \cdot c_{\mathrm{R}} \cdot c_{\mathrm{X}}
$$

Transfer führt im Allgemeinen zu einer Verringerung des mittleren Polymerisationsgrades des Polymerprodukts. Häufig wird der Transferkoeffizient in Relation zum Propagationskoeffizienten gestellt. Daraus ergibt sich die Transferkonstante $C_{\mathrm{tr}, \mathrm{X}}$.

$$
C_{\mathrm{tr}, \mathrm{X}}=\frac{k_{\mathrm{tr}, \mathrm{X}}}{k_{\mathrm{p}}}
$$

\subsubsection{Intramolekularer Transfer}

Der intramolekulare Transfer beschreibt eine Übertragung der Radikalfunktionalität innerhalb des Makroradikals. Dieser Effekt ist besonders ausgeprägt bei der Polymerisation von Acrylaten, wobei eine Wasserstoffabstraktion über einen sechsgliedrigen Übergangszustand, das sogenannte backbiting, stattfindet. Aus einem sekundären propagierenden Radikal (SPR) entsteht ein stärker stabilisiertes tertiäres Radikal (MCR, mid chain radical). Kontrolliert durch den Geschwindigkeitskoeffizienten $k_{\mathrm{bb}}$ wird die Radikalfunktionalität vom Kettenende auf die dritte Monomereinheit, durch eine konzertierte [1,5]Wasserstoffübetragunsreaktion, verschoben (s. Abbildung 3.4). 


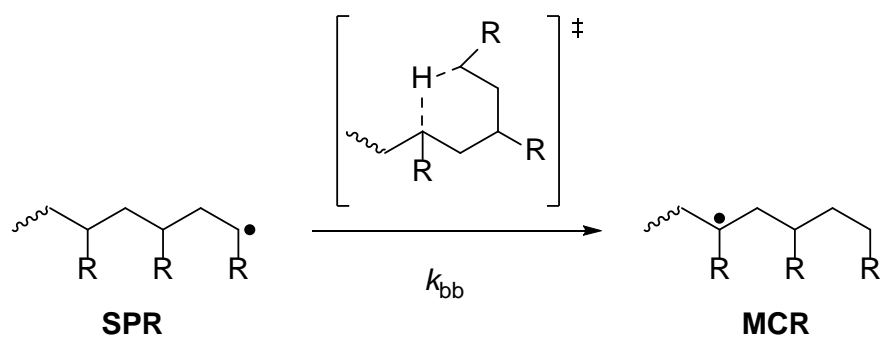

\section{Abbildung 3.4: Schematische Darstellung der backbiting Reaktion. R kennzeichnet eine Seitengruppe.}

Die Triebkraft der Reaktion ist, resultierend aus der sehr unterschiedlichen Stabilität von Edukt und Produkt, enthalpischer Natur. Daraus ergibt sich, dass weiteres backbiting ausgehend von einem MCR zu einer späteren Position statistisch betrachtet, aufgrund des geringen enthalpischen Gewinns, deutlich unwahrscheinlicher wird,. ${ }^{[47]}$ Experimentell wurde ein Anteil von Langkettenverzweigungen beobachtet, der zusätzlich auf einen entropischen Beitrag in der Reaktion schließen lässt. ${ }^{[48]}$ Backbiting ist eine irreversible Reaktion, MCRs können jedoch durch Kreuzpropagation wieder in SPRs überführt werden. ${ }^{[16]}$ Homoterminierung mit anderen MCRs und Kreuzterminierung mit SPRs ist weiterhin ein möglicher Reaktionsweg für diese Spezies. Eine komplette Übersicht der Reaktivität ist in Kapitel 7 dargestellt. Backbiting hat aufgrund der stark differierenden Reaktivität von SPRs und MCRs tiefgreifende Folgen für die Kinetik der betroffenen Systeme. Im Allgemeinen lässt sich, im Vergleich zu Vorhersagen die nur SPR-Kinetik berücksichtigen, eine Retardierung bei der Polymerisation von Acrylaten beobachten. ${ }^{[49]}$ Auf struktureller Ebene bedeutet backbiting die Entstehung von Kurzkettenverzweigungen innerhalb der Polymere, welche Auswirkungen auf die Eigenschaften des Produktes haben können.

Das Geschwindigkeitsgesetz für backbiting ist erster Ordnung und gegeben als:

$$
-\frac{\mathrm{d} c_{\mathrm{SPR}}}{\mathrm{d} t}=k_{\mathrm{bb}} \cdot c_{\mathrm{SPR}} .
$$

Über den Einfluss des backbiting auf die Kinetik der Reaktion unter quasistationäre Bedingungen wurde in der Literatur ausführlich 
berichtet. ${ }^{[50-53]}$ Hier soll nur ein kurzer Einblick in die Zusammenhänge der Geschwindigkeitskoeffizienten gegeben werden. Zunächst kann ein effektiver Propagationskoeffizient $k_{\mathrm{p}}^{\text {eff }}$ definiert werden. Dieser beschreibt die in einem Umsatz-Zeit-Experiment beobachtbare Monomeradditionsrate.

$$
k_{\mathrm{p}}^{\mathrm{eff}}=k_{\mathrm{p}}^{\mathrm{s}} \cdot\left(1-x_{\mathrm{MCR}}\right)+k_{\mathrm{p}}^{\mathrm{t}} \cdot x_{\mathrm{MCR}}
$$

Hier ist $k_{\mathrm{p}}^{\mathrm{s}}$ der Propagationskoeffizient der SPRs, $k_{\mathrm{p}}^{\mathrm{t}} \quad \operatorname{der}$ Propagationskoeffizient der MCRs und $x_{\text {MCR }}$ der Anteil an MCRs an der gesamten Radikalpopulation. Aufgrund von $k_{\mathrm{p}}^{\mathrm{s}}>k_{\mathrm{p}}^{\mathrm{t}}$ kann (3.25) vereinfacht werden und eine lineare Beziehung zwischen den Anteil an MCRs und der Retardierung der Reaktion aufgestellt werden:

$$
k_{\mathrm{p}}^{\mathrm{eff}}=k_{\mathrm{p}}^{\mathrm{s}} \cdot\left(1-x_{\mathrm{MCR}}\right)
$$

Durch Vernachlässigung der MCR Terminierung und der Annahme von Kreuzpropagation als einzigen MCR Abbauschritt, kann der Anteil an MCRs im System wie folgt beschrieben werden:

$$
x_{\mathrm{MCR}}=\frac{k_{\mathrm{bb}}}{k_{\mathrm{bb}}+k_{\mathrm{p}}^{\mathrm{t}} \cdot c_{\mathrm{M}}} \text {. }
$$

Analog zu (3.26) lässt sich auch für die Terminierung ein effektiver Koeffizient aufstellen. Hierbei kennzeichnet $k_{\mathrm{t}}^{\text {ss }}$ den Terminierungskoeffizient zweier SPRs, $k_{\mathrm{t}}^{\text {st }}$ den eines SPRs und eines MCRs und $k_{\mathrm{t}}^{\mathrm{tt}}$ den zweier MCRs. 


$$
\begin{gathered}
k_{\mathrm{t}}^{\mathrm{eff}}=k_{\mathrm{t}}^{\mathrm{ss}} \cdot\left(1-x_{\mathrm{MCR}}\right)^{2}+k_{\mathrm{t}}^{\mathrm{st}} \cdot\left(1-x_{\mathrm{MCR}}\right) \cdot x_{\mathrm{MCR}}+k_{\mathrm{t}}^{\mathrm{tt}} \\
\cdot\left(x_{\mathrm{MCR}}\right)^{2}
\end{gathered}
$$

Die Polymerisationsrate des Systems kann mit den eingeführten Koeffizienten beschrieben werden.

$$
R_{\mathrm{p}}=-\frac{\mathrm{d} c_{\mathrm{M}}}{\mathrm{d} t}=\frac{k_{\mathrm{p}}^{\mathrm{eff}}}{\sqrt{k_{\mathrm{t}}^{\mathrm{eff}}}} \cdot \sqrt{k_{\mathrm{d}} \cdot f \cdot c_{\mathrm{I}}} \cdot c_{\mathrm{M}}
$$

\subsection{Propagationskoeffizienten mittels PLP-SEC}

Die PLP-SEC Methode wurde von Olaj et al. eingeführt und basiert auf der Synthese von Polymermaterial durch periodische Bestrahlung mittels Laserpulsen mit kurzen Pulsbreiten $(<20 \mathrm{~ns}) .{ }^{[2,3]}$ Prinzipiell ist Terminierung innerhalb des Experiments jederzeit möglich, jedoch aufgrund der erhöhten Radikalkonzentration zum Zeitpunkt der Laserbestrahlung am wahrscheinlichsten. Die anschließende Analyse der Molmassenverteilungen mittels SEC erlaubt die Korrelation zwischen Reaktionszeit $(t)$ und Polymerisationsgrad $\left(L_{b}\right)$ der entstandenen Polymere und daraus die Bestimmung von $k_{\mathrm{p}}$. Dazu wird der Polymerisationsgrad der Laserinitiierten und -terminierten Ketten durch die Wendepunkte (point of inflection, POI) auf der niedermolekularen Seite der Molmassenverteilung der PLP Strukturen bestimmt. Der Faktor $b$ berücksichtigt Ketten die nicht durch den konsekutiven Laserpuls, sondern bei den darauffolgenden Laserpulsen terminiert wurden. Die der Technik zugrundeliegende Theorie und die sich daraus ergebenden Limitierungen sind ausführlich in der Literatur beschrieben. ${ }^{[54,55]}$ 


$$
L_{b}=k_{\mathrm{p}} \cdot c_{\mathrm{M}} \cdot t \cdot b
$$

Mithilfe dieser Technik konnten verlässliche Propagationskoeffizienten für eine Reihe von Monomeren in Substanz wie Styrol, Methacrylate und Acrylate bestimmt werden. ${ }^{[4,6,56,57]}$

Die Analyse des Polymerprodukts erfolgt durch SEC und basiert auf der säulenchromatographischen Trennung der Moleküle nach hydrodynamischen Radius. Erfolgt die Detektion durch Konzentrationsdetektoren wie Brechungsindex- oder UV-Detektoren muss der Aufbau durch Elutionsvolumen-zu-Molmasse-Korrelationen von bekannten Proben kalibriert werden. Solche Kalibrierungsstandards sind nur für eine limitierte Auswahl an Polymeren verfügbar. Zur Analyse anderer Polymere kann das Prinzip der universellen Kalibrierung verwendet werden. ${ }^{[58]}$ Dazu wird das hydrodynamische Volumen $V_{\mathrm{h}}$ ins Verhältnis zur intrinsischen Viskosität $[\eta]$ und Molmasse $M$ des Polymers gesetzt.

$$
V_{\mathrm{h}}=\frac{[\eta] \cdot M}{2,5 \cdot N_{\mathrm{A}}}
$$

Die intrinsische Viskosität leitet sich aus der Mark-Houwink-Gleichung ab, welche mithilfe der Mark-Houwink-Koeffizienten $K$ und $a$ das Verhalten von Polymeren in Lösungen beschreibt.

$$
[\eta]=K \cdot M^{a}
$$

Sind die Mark-Houwink-Koeffizienten für das zu messende Polymer bekannt kann die Molmassenverteilung, welche aus Messungen von Kalibrierungsstandards gewonnen wurde, mittels (3.34) in eine absolute Molmassenverteilung umgerechnet werden. Dabei wird angenommen, dass die Elutionszeit nur abhängig vom hydrodynamischen Volumen und nicht 
von der Art des Polymers ist. Somit gilt für zwei Polymere mit gleichem hydrodynamischen Volumen:

$$
\begin{gathered}
K_{1} \cdot M_{1}^{a_{1}+1}=K_{2} \cdot M_{2}{ }^{a_{2}+1} \\
\log \left(M_{2}\right)=\frac{1}{1+a_{2}} \log \left(\frac{K_{1}}{K_{2}}\right)+\frac{1+a_{1}}{1+a_{2}} \log \left(M_{1}\right)
\end{gathered}
$$

Der Index 2 kennzeichnet das zu untersuchende Polymer, während 1 das Polymer aus der Kalibrierung bezeichnet.

\subsection{Analyse der Kettenlängenabhängigkeit der Terminierung mittels SP-PLP-ESR}

Die SP-PLP-ESR Methode ist die direkteste Methode die Kettenlängenabhängigkeit des Terminierungsprozesses in radikalischen Polymerisationen zu messen. Durch Messung des Verlaufes der Radikalkonzentration nach nahezu instantaner Initiierung durch den Laserpuls kann eine vollständige Beschreibung der Terminierung nach dem composite model erreicht werden. Dank des homogenen Wachstums der Ketten kann die Zeitachse in eine Kettenlängenachse überführt werden.

$$
i=k_{\mathrm{p}} \cdot c_{\mathrm{M}} \cdot t
$$

Das mittlere Zeitintervall der Propagation $t_{\mathrm{p}}$ kann somit derart ausgedrückt werden: 


$$
t_{\mathrm{p}}=\frac{1}{k_{\mathrm{p}} \cdot c_{\mathrm{M}}} .
$$

Werden die Ausdrücke (3.35) und (3.37) auf die Kurz- und Langkettenbeschreibung nach dem composite model (3.18) und (3.19) angewendet und dies in das Geschwindigkeitsgesetz der Terminierung (3.4) eingesetzt, wird die Abnahme der Radikalkonzentration nach einem Laserpuls für den Kurzketten- und Langkettenbereich erhalten.

$$
\begin{gathered}
\frac{c_{\mathrm{R}}}{c_{\mathrm{R}}^{0}}=\left(\frac{2 \cdot k_{\mathrm{t}}^{1,1} \cdot c_{\mathrm{R}}^{0} \cdot t_{\mathrm{p}}^{\alpha_{\mathrm{s}}}}{1-\alpha_{\mathrm{s}}} \cdot t_{\mathrm{p}}^{\left(1-\alpha_{\mathrm{s}}\right)}+1\right)^{-1} \\
\frac{c_{\mathrm{R}}}{c_{\mathrm{R}}^{0}}=\left(\frac{2 \cdot k_{\mathrm{t}}^{0} \cdot c_{\mathrm{R}}^{0} \cdot t_{\mathrm{p}}^{\alpha_{1}}}{1-\alpha_{\mathrm{l}}} \cdot t_{\mathrm{p}}^{\left(1-\alpha_{\mathrm{l}}\right)}+1\right)^{-1} \\
\log \left(\frac{c_{\mathrm{R}}^{0}}{c_{\mathrm{R}}}-1\right)=\log (C)+(1-\alpha) \cdot \log (t)
\end{gathered}
$$

Eine doppeltlogarithmische Auftragung nach (3.39) von (3.37) und (3.38) erlaubt theoretisch die Bestimmung von $\alpha_{\mathrm{s}}$ und $\alpha_{\mathrm{l}}$ aus der Steigung und von $k_{\mathrm{t}}^{1,1}$ und $k_{\mathrm{t}}^{0}$ aus dem Ordinatenabschnitt bei Kenntnis von $c_{\mathrm{R}}^{0}$.

Für den Kurzkettenbereich stellte sich diese Herangehensweise aufgrund der Vernachlässigung des Initiatorfragments als ungenügend heraus. ${ }^{[59]}$ Russel et al. schlugen eine Erweiterung von (3.35) vor. ${ }^{[60]}$

$$
i=k_{\mathrm{p}} \cdot c_{\mathrm{M}} \cdot t+1
$$

Mit analoger Vorgehensweise wie für (3.37) lässt sich ein neuer Term für den Kurzkettenbereich aufstellen. 


$$
\frac{c_{\mathrm{R}}}{c_{\mathrm{R}}^{0}}-1=\frac{2 \cdot k_{\mathrm{t}}^{1,1} \cdot c_{\mathrm{R}}^{0} \cdot\left(\left(k_{\mathrm{p}} \cdot c_{\mathrm{M}} \cdot t+1\right)^{1-\alpha_{\mathrm{s}}}-1\right)}{k_{\mathrm{p}} \cdot c_{\mathrm{M}} \cdot\left(1-\alpha_{\mathrm{s}}\right)}
$$

Die beste Übereinstimmung der experimentellen Daten mit Modellparametern durch Analyse des Langkettenbereiches mittels doppeltlogarithmischer Auftragung nach (3.39) und der nichtlinearen Anpassung von (3.41) im Kurzkettenbereich erhalten. Eine ausführliche Beschreibung der Vorgehensweise zur Bestimmung der gesamten Parameter der kettenlängenabhängigen Terminierung wird in Kapitel 10 ausführlich vorgestellt. 


\section{Experimenteller Teil}

\subsection{Chemikalien}

\subsubsection{Initiatoren}

2-Hydroxy-2-methyl-1-phenylpropan-1-on (Darocure 1173)

2-Hydroxy-2-methyl-1-phenylpropan-1-on $\left(M=164,2 \mathrm{~g} \cdot \mathrm{mol}^{-1}\right.$, Sigma Aldrich, $97 \%$ ) wurde ohne weitere Aufarbeitung verwendet.

\section{2,2-Dimethoxy-1,2-diphenylethan-1-on (DMPA)}

2,2-Dimethoxy-1,2-diphenylethan-1-on $\quad\left(M=256,30 \mathrm{~g} \cdot \mathrm{mol}^{-1}\right.$, Sigma Aldrich, 99\%) wurde ohne weitere Aufarbeitung verwendet.

\section{2,2'-Azobis(2-methylpropionitril) (AIBN)}

2,2'-Azobis(2-methylpropionitril) ( $M=164,21 \mathrm{~g} \cdot \mathrm{mol}^{-1}$, Sigma Aldrich, 98\%) wurde durch Umkristallisation aus Aceton gereinigt.

\subsubsection{Monomere}

\section{2-Ethylhexylmethacrylat (EHMA)}

2-Ethylhexylmethacrylat $\left(M=198,30 \mathrm{~g} \cdot \mathrm{mol}^{-1}\right.$, Sigma Aldrich, $98 \%$, 50 ppm Hydrochinonmonomethylether) wurde mit Inhibitorremover (Aldrich) säulenchromatographisch gereinigt und direkt weiterverwendet. 


\section{6-(Methacryloyloxy)-hexyl-4'-pentyl-[1,1'-bi(cyclohexan)]-4-carboxylat (MA-HPBCHC)}

6-(Methacryloyloxy)-hexyl-4'-pentyl-[1,1'-bi(cyclohexan)]-4-carboxylat ( $M$ = 448,69 $\mathrm{g} \cdot \mathrm{mol}^{-1}$ ) wurde von Merck KGaA im Rahmen einer Kooperation zur Verfügung gestellt. Das Monomer wurde mit Inhibitorremover (Aldrich) säulenchromatographisch gereinigt und direkt weiterverwendet.

\section{6-(Acryloyloxy)-hexyl-4'-pentyl-[1,1'-bi(cyclohexan)]-4-carboxylat} (A-HPBCHC)

6-(Acryloyloxy)-hexyl-4'-pentyl-[1,1'-bi(cyclohexan)]-4-carboxylat ( $M=$ 434,66 $\mathrm{g} \cdot \mathrm{mol}^{-1}$ ) wurde von Merck KGaA im Rahmen einer Kooperation zur Verfügung gestellt. Das Monomer wurde mit Inhibitorremover (Aldrich) säulenchromatographisch gereinigt und direkt weiterverwendet.

\section{6-((2-Fluoroacryloyl)oxy)-hexyl-4'-pentyl-[1,1'-bi(cyclohexan)]-4- carboxylat (FA-HPBCHC)}

6-((2-Fluoroacryloyl)oxy)-hexyl-4'-pentyl-[1,1'-bi(cyclohexan)]-4carboxylat $\left(M=452,65 \mathrm{~g} \cdot \mathrm{mol}^{-1}\right)$ wurde von Merck KGaA im Rahmen einer Kooperation zur Verfügung gestellt. Das Monomer wurde mit Inhibitorremover (Aldrich) säulenchromatographisch gereinigt und direkt weiterverwendet.

\section{6-((2-Methylenebutanoyl)oxy)hexyl-4'-pentyl-[1,1'-bi(cyclohexan)]-4- carboxylat (EA-HPBCHC)}

6-((2-Methylenebutanoyl)oxy)hexyl-4'-pentyl-[1,1'-bi(cyclohexan)]-4carboxylat $\left(M=462,72 \mathrm{~g} \cdot \mathrm{mol}^{-1}\right)$ wurde von Merck KGaA im Rahmen einer Kooperation zur Verfügung gestellt. Das Monomer wurde mit Inhibitorremover (Aldrich) säulenchromatographisch gereinigt und direkt weiterverwendet.

\subsubsection{Weitere Chemikalien}

\section{4-Cyano-4-[(dodecylsulfanylthiocarbonyl)sulfanyl]pentansäure (CDSTSP)}

4-Cyano-4-[(dodecylsulfanylthiocarbonyl)sulfanyl]pentansäure ( $M=403,67 \mathrm{~g} \cdot \mathrm{mol}^{-1}$, Sigma Aldrich, $97 \%$ ) wurde ohne weitere Reinigung verwendet. 


\section{2-(Dodecylthiocarbonothioylthio)-2-methylpropionsäure (DDMAT)}

2-(Dodecylthiocarbonothioylthio)-2-methylpropionsäure

( $M=364,63 \mathrm{~g} \cdot \mathrm{mol}^{-1}$, Sigma Aldrich, $98 \%$ ) wurde ohne weitere Reinigung verwendet.

\section{Hydrochinonmonomethylether (MEHQ)}

Hydrochinonmonomethylether $\left(M=124,14 \mathrm{~g} \cdot \mathrm{mol}^{-1}\right.$, Fluka) wurde ohne weitere Aufarbeitung verwendet

\section{2,2,6,6-Tetramethylpiperidinyloxyl (TEMPO)}

2,2,6,6-Tetramethyl-1-piperidinyloxyl (TEMPO, $M=156,26 \mathrm{~g} \cdot \mathrm{mol}^{-1}$, Sigma Aldrich, $99 \%$ ) wurde ohne weitere Reinigung verwendet.

\section{4-Oxo-2,2,6,6-tetramethyl-1-piperidinyloxy (TEMPONE)}

4-Oxo-2,2,6,6-tetramethyl-1-piperidinyloxy ( $M=170,23 \mathrm{~g} \cdot \mathrm{mol}^{-1}$, Sigma Aldrich, $99 \%$ ) wurde ohne weitere Reinigung verwendet.

\section{Toluol}

Toluol (puriss. p.a., $\geq 99,7 \%$ (GC), Sigma Aldrich) wurde ohne weitere Reinigung verwendet.

\section{BL-087}

BL-087 ist eine nematische Flüssigkristallmischung welche von Merck KGaA im Rahmen einer Kooperation zur Verfügung gestellt wurde. Der Klärpunkt der reinen Lösung liegt bei $89^{\circ} \mathrm{C}$.

\section{R-5011}

R-5011 ist ein chiraler Dotierstoff der von Merck KGaA im Rahmen einer Kooperation zur Verfügung gestellt wurde.

\subsection{Dichtemessungen}

Die Dichtemessungen wurden nach dem Prinzip eines oszillierenden URohrs durchgeführt. Das Dichtemeter besteht aus einer Messeinheit (Anton Paar, DMA 60 und Anton Paar, DMA 602TP) und einer Hochtemperaturzelle (Anton Paar, DMA $602 \mathrm{H}$, Duran ${ }^{\circledR}$ $50,-10 \leq T \leq 150^{\circ} \mathrm{C}$ ). Die Temperatur innerhalb des U-Rohrs wurde mittels eines digitalen Thermometers gemessen. 
Die Dichte ließ sich aus folgender Gleichung ermitteln.

$$
\rho=\frac{1}{\mathrm{~A}}\left(G^{2}-\mathrm{B}\right)
$$

Hierbei handelt es sich bei $G$ um die natürliche Frequenz und bei $A$ und $B$ um Gerätekonstanten die aus den folgenden Gleichungen berechnet wurden. Die benötigten Dichten für Luft und Wasser wurden aus der Literatur entnommen. ${ }^{[61]}$

$$
\begin{gathered}
\mathrm{A}=\frac{G_{\mathrm{H}_{2} \mathrm{O}}^{2}-G_{\mathrm{Luft}}^{2}}{\rho_{\mathrm{H}_{2} \mathrm{O}}-\rho_{\mathrm{Luft}}} \\
\mathrm{B}=T_{\mathrm{Luft}}^{2}-\left(A \cdot \rho_{\mathrm{Luft}}\right)
\end{gathered}
$$

\subsection{FT-NIR Spektroskopie}

Die Fourier-Transformation-Nah-Infrarot-(FT-NIR) Spektroskopie wurde verwendet um durch Abnahme des ersten Obertons der antisymmetrischen $\mathrm{CH}$-Streckschwingung der Methylidengruppe, bei etwa 6050 bis $6300 \mathrm{~cm}^{-1}$, die Abnahme der Monomerkonzentration zu bestimmen. Aus dem integrierten Lambert-Beerschen-Gesetz ergibt sich der Monomer-zuPolymer Umsatz wie folgt.

$$
A_{\text {int }}=\int A_{10}(\tilde{v}) d \tilde{v}
$$




$$
X=1-\frac{A_{\text {int }}(t)}{A_{\text {int }}(t=0)}
$$

Hierbei handelt es sich bei $A_{10}(\tilde{v})$ um die dekadische Absorbanz bei der Wellenzahl $\tilde{v}$ und bei $A_{\text {int }}(t)$ um der zum Zeitpunkt $t$ über ein Wellenzahlbereich integrierte dekadische Absorbanz.

Die Messungen wurden an einem Spektrometer (IFS 88, Bruker) mit einer Quecksilber-Halogen Lampe, ein siliconbeschichtetem Calciumfluorid Strahlenteiler und einem InSb Detektor durchgeführt. Zur Bestimmung der Umsätze in SP-PLP-ESR Experimenten wurden die ESR-Flachzellen in der Messkammer platziert. Die Spektren wurden mittels der Software Opus (Bruker Optic, version 6.0) aufgenommen und ausgewertet.

\subsection{PLP-SEC}

Die Reaktionslösung wurde in eine Durchflussküvette (Starna, 65.14/Q/10, Spectrosil $^{\circledR} \quad$ Quarzglas, Transmission von mindestens 0,8 für $192 \mathrm{~nm}-2700 \mathrm{~nm}$, optische Weglänge von $10 \mathrm{~mm}$ ) gegeben und mindestens 5 Minuten mit Argon durchströmt um inhibierende Reste an Sauerstoff zu entfernen. Nach Verschließen der Küvette wurde diese mittels eines externen Wärmebades (Haake K, Haake F3) 10 Minuten temperiert. Das Wärmebad verwendete ein 4:1 Gemisch von Ethylenglycol/Wasser als Wärmetransportflüssigkeit. Anschließend wurde die Probe mittels eines ATLEX-I Lasers (ATL Lasertechnik GmbH, Pulsbreite 20 ns, maximale Pulsenergie 7 mJ, maximale Pulsfrequenz $1000 \mathrm{~Hz}$, mit einer Wellenlänge $351 \mathrm{~nm}$ (XeF-Linie)) bestrahlt. Hierbei wurden Pulsfrequenzen von $1 \mathrm{~Hz}$ bis $1000 \mathrm{~Hz}$ verwendet. Eine detaillierte Beschreibung des Aufbaus der Apparatur findet sich in der Literatur. ${ }^{[12]}$

Nach Durchführung der Pulslaser-induzierten Polymerisation wurde der Probe Hydrochinon zur Inhibierung weiterer Polymerisation hinzugegeben und das Lösungsmittel wurde im Vakuum entfernt. Die Analyse mittels SEC ist in Abschnitt 4.5 beschrieben. 


\subsection{SEC}

Die Größenausschlusschromatographische (SEC) der hergestellten Polymere wurde bei $35{ }^{\circ} \mathrm{C}$ mit Tetrahydrofuran als Eluent $(1 \mathrm{~mL} / \mathrm{min}$ Fließrate) und Toluol als interner Standard durchgeführt. Hierbei wurde ein SEC System verwendet welches aus einer HPLC Pumpe (Waters, Modell 515), einem Autosampler (Jasco, AS-2055-plus), drei PSS SDV Säulen (5 $\mu \mathrm{m}$ Partikelgröße; Porengrößen von $10^{5} \AA, 10^{3} \AA$ und $10^{2} \AA$ ), einem Brechungsindexdetektor (Waters, Modell 2410), einem UV/VIS-Detektor (Viskotek VE 3210). und einem Lichtstreudetektor (PSS, SLP 7000) bestand. Der Aufbau wurde mithilfe von niedrig-dispersen Poly(MMA) und Poly(Styrol) Standards kalibriert ( $M=800$ bis $200000 \mathrm{~g} \mathrm{~mol}^{-1}$, PSS).

\subsection{Elektronenspinresonanz}

\subsubsection{Experimenteller Aufbau}

Die Experimente im Rahmen dieser Arbeit wurden an zwei unterschiedlichen Geräten durchgeführt. Der prinzipielle Aufbau wird im Folgenden beschrieben.

Die Mikrowellenbrücke beherbergt gleichzeitig Quelle und Detektor der Mikrowellenstrahlung. Diese wird in den Hohlraumresonator gelenkt, welche die schwachen Signale der Probe verstärkt und durch ihren $Q$ Wert charakterisiert werden kann, der ein Maß für die Effizienz der Speicherung der Mikrowellenenergie ist. Dementsprechend bedeutet ein hoher $Q$ Wert eine hohe Sensitivität des Spektrometers. Der Hohlraumresonator besitzt ein Gitter welches die Bestrahlung der Probe durch externe Quellen erlaubt. Durch ein ADC Signalkanal (SC) wird die Aufnahme von Signalintensität vs. Magnetfeld sowie Signalintensität vs. Zeit bei konstantem Magnetfeld ermöglicht. Die höchstmögliche Zeitauflösung beträgt $10 \mu \mathrm{s}$.

Die Synchronisation des Lasers und des Spektrometers erfolgte über einen Pulsgenerator (Quantum Composers 9314, Scientific Instruments). Die Temperaturkontrolle erfolgte durch einen Stickstoffstrom der durch den Hohlraumresonator geleitet wurde (ER 4131VT, Bruker). Die Konsole beherbergt die Signalverarbeitungseinheiten und Kontrolle der Elektronik. 
Die Steuerung des Spektrometers erfolgte durch die Software XEPR (Bruker).

Beim ersten verwendeten Spektrometer handelt es sich um ein X-Band Bruker Elexsys E 500 CW-ESR. Hierbei wurde ein SHQE-W1 Hohlraumresonator eingebaut und ein XeF Excimer Laser (Lambda Physik, COMPex 102, maximale Pulsfrequenz $20 \mathrm{~Hz}$ ) zur Bestrahlung verwendet. Der Laser operiert bei einer Emissionswellenlänge von $351 \mathrm{~nm}$. Weiterhin ist der Aufbau durch ein fast digitizer acquisition board ergänzt, welcher die Zeitauflösung auf 500 ns verbessert. Das Spektrometer befindet sich im Institut für Anorganische Chemie in Göttingen.

Das zweite verwendete Gerät ist ein X-Band Bruker Elexsys II E 500T CW-ESR. Hierbei wurde ein ER 41122SHQE-LC Hohlraumresonator eingebaut und ein XeF Excimer Laser (Lambda Physik, LPX 210i, maximale Pulsfrequenz 100). Der Laser operiert bei einer Emissionswellenlänge von $351 \mathrm{~nm}$. Der eingebaute Hohlraumresonator besitzt einen besseren $Q$ Wert und weist somit ein besseres Signal-zuRausch Verhältnis als das zuvor genannte Gerät.

\subsubsection{Probenvorbereitung und Messdurchführung}

Zur Probenvorbereitung wurden das gereinigte Monomer und das verwendete Lösungsmittel durch mehrere konsekutive Einfrier-, Evakuier-, Auftau-Zyklen entgast. Alle Proben wurden anschließend unter Argon Atmosphäre in einer Handschuhbox mit Initiator versetzt und mit einer Mikroliterspritze in ESR-Flachzellen abgefüllt (Durchmesser $1 \mathrm{~mm}$, Suprasil $^{\circledR}$ ). Die Proben wurden mit PARAFILM abgedichtet und unter Lichtausschluss bis zur Messung aufbewahrt.

Spektren wurden, wenn nicht anders angegeben, mit einer Modulationsfrequenz von $100 \mathrm{kHz}$ und ein Receiver Gain von 60 gemessen. Modulationsamplituden wurden zwischen $1 \mathrm{G}$ und $5 \mathrm{G}$ variiert. Die Mikrowellenenergie wurde je nach Probe zwischen $2 \mathrm{~mW}$ und $20 \mathrm{~mW}$ gesetzt, wobei für jedes System überprüft wurde, dass das Signal sich nicht im Saturationsbereich befand. 
Die typische Durchführung eines SP-PLP-ESR Experiments wird im Folgenden beschrieben. Die Probe wird im Hohlraumresonator platziert und 5 Minuten bis zum Temperaturausgleich gewartet. Anschließend wird ein ESR-Spektrum durch kontinuierliche Laserbestrahlung aufgenommen (typischerweise $20 \mathrm{~Hz}$ ), um die geeigneten Magnetfeldpositionen für eine Einzelpulsmessung zu bestimmen. Anschließend werden die Einzelpulsmessungen bei konstantem magnetischem Feld an der ausgewählten Position durchgeführt. Um eine ausreichendes Signal-zuRausch Verhältnis zu gewährleisten wurden bis zu 50 Scans gemittelt. Hierbei wurde sichergestellt, dass niemals über $5 \%$ Umsatz polymerisiert wurde. Umsätze wurden mittels FT-NIR bestimmt (siehe Abschnitt 4.3).

\subsubsection{Kalibrierung}

Zur Bestimmung von absoluten Radikalkonzentrationen muss das ESRSpektrometer kalibriert werden. Die Polarität der Probe, Probenform und Menge haben Einfluss auf die Signalintensität, deshalb muss die Kalibrierlösung exakt den Messlösungen entsprechen. Dementsprechend wurde den Kalibrierlösungen in unterschiedlichen Konzentrationen TEMPO hinzugefügt. Anschließend wurden Spektren jeder Lösung aufgenommen.

In der ESR-Spektroskopie wird aufgrund der Modulation des Signals die erste Ableitung der Absorbanz aufgenommen. Dies bedeutet, dass das Doppelintegral mit der Konzentration der Spezies korreliert werden kann. Diese Korrelation ist unabhängig von der Art des Radikals. Dementsprechend kann mittels der Kalibrierung mit TEMPO der Proportionalitätsfaktor $h_{1}$ ermittelt werden.

$$
c_{\mathrm{R}}=h_{1} \iint I_{\mathrm{SC}}
$$




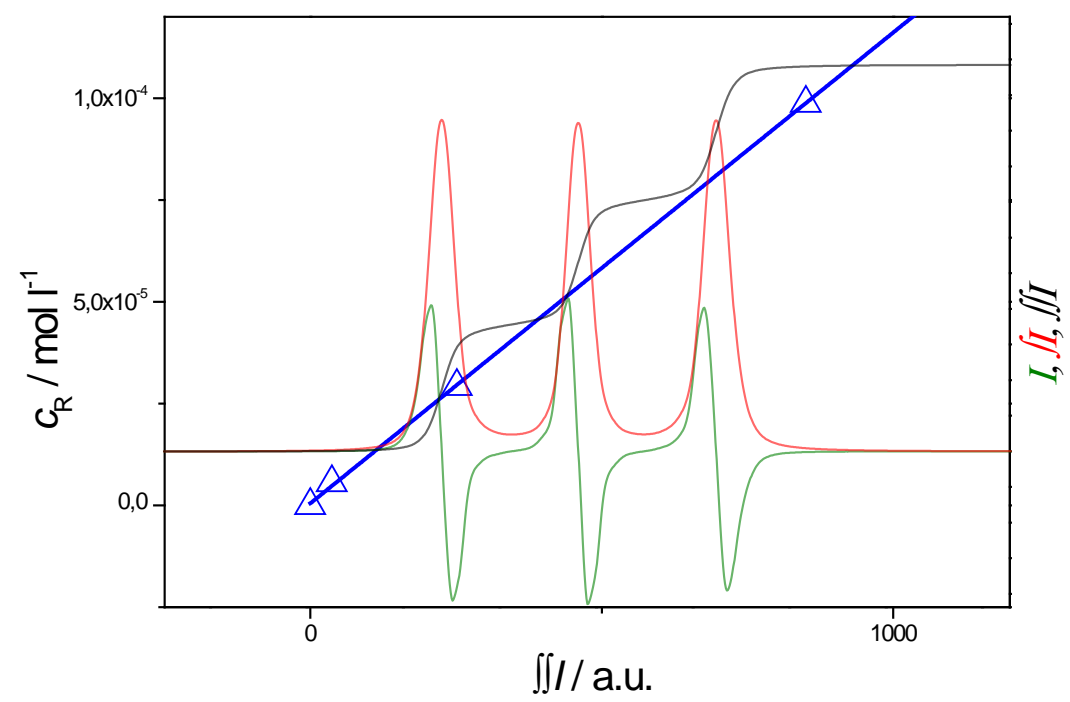

Abbildung 4.1: Bestimmung der Kalibrierkonstante $h_{1}$. Dazu ist die TEMPO Konzentration gegen das Doppelintegral der ESR-Spektren aufgetragen. Die Steigung der Linearisierung ergibt $h_{1}$. Ein repräsentatives Spektrum für TEMPO (grün), sowie das Integral (rot) und Doppelintegral (schwarz) sind weiterhin aufgetragen. Aufgenommen bei $293 \mathrm{~K}$ in $10 \mathrm{wt} \%$ EHMA in BL-087.

Bei Einzelpulsmessungen, die im Rahmen von SP-PLP-ESR durchgeführt werden, wird die Intensität eines Peaks zeitaufgelöst verfolgt, d.h. die Messung erfolgt bei konstantem magnetischem Feld $\left(B_{\mathrm{x}}\right)$. Um diese Intensitätsverläufe in Konzentrationsverläufe umzurechnen bedarf es eines weiteren Kalibriervorganges. Hierzu muss das Verhältnis von Doppelintegral zu Peakintensität des Spektrums durch Korrelation ermittelt werden.

$$
\iint I_{\mathrm{SC}}=h_{2} I_{\mathrm{SC}}\left(B_{\mathrm{x}}\right)
$$




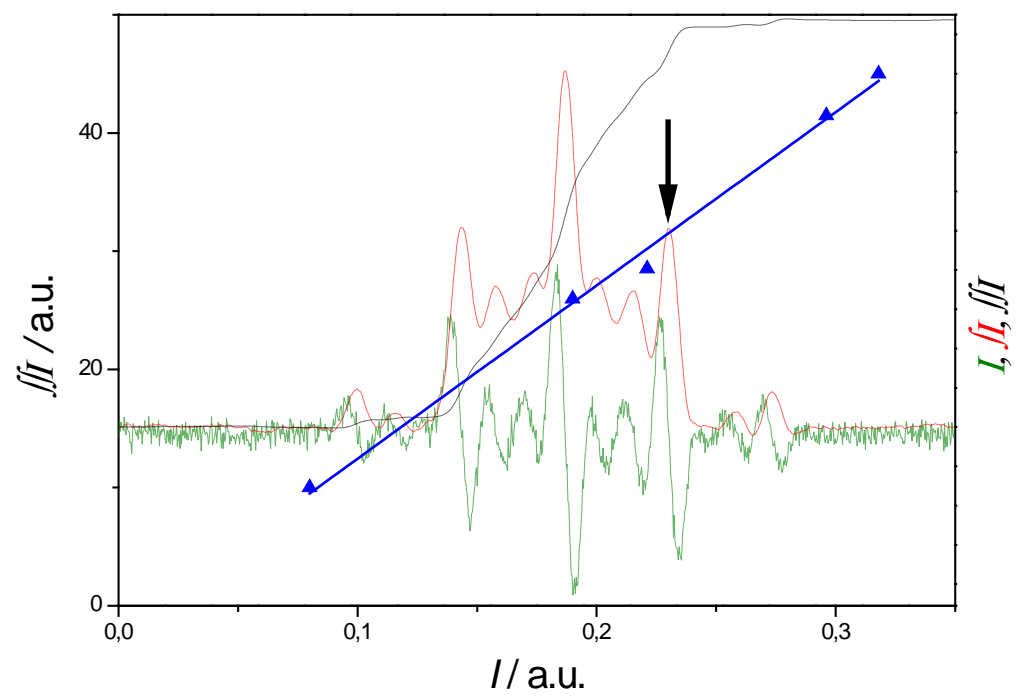

Abbildung 4.2: Auftragung des Verhältnisses von Doppelintegral zu Peakhöhe der ESR Spektren zur Bestimmung der Kalibrierkonstante $h_{2}$ für 10 wt\% EHMA in BL-087 bei 293,15 K. Der verwendete Peak ist mit einem Pfeil gekennzeichnet. In Grün ist das experimentelle Spektrum, in Rot das integrierte Spektrum und in Schwarz das Doppelintegral dargestellt.

Somit lässt sich für ein Einzelpulsexperiment die Radikalkonzentration mittels folgenden Zusammenhangs bestimmen.

$$
c_{\text {rad }}(t)=h_{1} \cdot h_{2} \cdot I_{S C}\left(B_{x}\right)(t)
$$




\section{Mark-Houwink-Parameter}

\subsection{Einleitung}

Die Notwendigkeit der Mark-Houwink-Parameter zur Durchführung von PLP-SEC-Experimenten wurde bereits in Abschnitt 3.6 besprochen. Für keines der im Rahmen dieser Arbeit untersuchten Monomere wurden Mark-Houwink-Parameter in der Literatur berichtet. Dementsprechend wurden diese für 6-(Methacryloyloxy)-hexyl-4'-pentyl-[1,1'bi(cyclohexan)]-4-carboxylat (MA-HPBCHC), 6-(Acryloyloxy)-hexyl-4'pentyl-[1,1'-bi(cyclohexan)]-4-carboxylat (A-HPBCHC) und 6-((2Fluoroacryloyl)oxy)-hexyl-4'-pentyl-[1,1'-bi(cyclohexan)]-4-carboxylat (FA-HPBCHC) bestimmt. Dazu wurden durch Reversible-AdditionFragmentation-chain-Transfer-(RAFT-) Polymerisation niedrigdisperse Proben hergestellt. Diese Proben wurden durch SEC in Kombination mit einem Brechungsindexdetektor charakterisiert und dessen relative Molmassenverteilungen in Bezug auf den Standard Poly(MMA) bestimmt. Durch eine weitere Detektionsmethode, in jedem Unterkapitel jeweils beschrieben, wurden die jeweiligen absoluten Molmassen dieser Proben bestimmt. Durch doppellogarithmische Auftragung von (3.33) wurden aus dem Ordinatenabschnitt und der Steigung $K$ und $\alpha$ bestimmt. Hierbei ist zu beachten, dass die Mark-Houwink-Beziehung (3.32) sich auf durch Viskosimetrie bestimme Molmassen bezieht. Diese werden in der Praxis oft durch das Gewichtsmittel der Molmasse $\bar{M}_{w}$ angenähert. ${ }^{[62]}$ Dementsprechend wurden zur Bestimmung der Mark-HouwinkKoeffizienten gewichtsmittlere Molmassen mittels der zuvor erwähnten doppeltlogarithmischen Auftragung verwendet. Die Kalibrierparameter 
wurden aus den Angaben des Herstellers der Standards (PSS) entnommen: $K_{\text {Poly (MMA) }}=0,01298 \mathrm{~mL} \mathrm{~g}^{-1}$ und $a_{\text {Poly(MMA) }}=0,688$.

\subsection{MA-HPBCHC}

Die Polymerisation von MA-HPBCHC (siehe Abbildung 5.1) wurde in Toluol durch Zugabe von AiBN und 4-Cyano-4[(dodecylsulfanylthiocarbonyl)sulfanyl]pentansäure (CDSTSP) durchgeführt. CDSTSP ist ein Literaturbekanntes RAFT-Agens. ${ }^{[63,64]}$ Bei der Polymerisation von Methylmethacrylat konnten in der Vergangenheit geringe Dispersitäten $(\bigoplus<1,2)$ der Produkte erreicht werden in Verknüpfung mit einer guten Übereinstimmung zwischen theoretischen und experimentellen Molmassen. ${ }^{[65]}$ Dies lässt eine gute Eignung zur Kontrolle der Polymerisation von MA-HPBCHC erwarten, da es sich ebenfalls um ein Methacrylat handelt. Zur Herstellung der Polymerproben wurde das Monomer in Toluol gelöst (40 wt\%) und mit CDSTSP und AiBN im festen Verhältnis der letzteren von 5:1 versetzt. Das Verhältnis von Monomer zu CDSTSP wurde variiert, um Polymere verschiedener Kettenlängen zu erzeugen. Die Polymerisation wurde bis zu 48 h durchgeführt, um hohe Umsätze $(X>0,8)$ zu gewährleisten.<smiles>C=C(C)C(=O)OCCCCCCOC(=O)C1CCC(C2CCC(CCCCC)CC2)CC1</smiles>

\section{MA-HPBCHC}

\section{Abbildung 5.1: Strukturformel von MA-HPBCHC.}

Die Polymerprodukte zeigten Dispersitäten unter 1,25 und eine Korrelation zwischen Kettenlänge und Verhältnis von Monomer zu RAFT-Agens. Aufgrund der sehr ausführlichen Reinigung, zur Gewährleistung einer genauen Charakterisierung durch Lichtstreuung, war ein Verlust an Produkt unvermeidbar. Aus Gründen der Messgenauigkeit muss somit ein Vergleich der theoretischen und experimentellen Kettenlängen der Polymere hier ausbleiben. Der Beweis einer Kontrolle der Reaktion ist für den Rahmen dieser Arbeit obsolet, die einzige Relevanz ist das Vorhandensein von 
niedrigdispersen Proben zur Ermittlung der Mark-Houwink-Koeffizienten. In Tabelle 5.1 sind die gewichtsmittleren Molmassen der Polymere aufgelistet welche durch zwei SEC Methoden bestimmt wurden. Für die gleiche Probe wurde zunächst mittels eines Brechungsindexdetektors und der durch Poly(MMA) kalibrierten Säule eine relative Molmassemassenverteilung bestimmt. Anschließend wird die absolute Molmassenverteilung durch Kopplung eines Brechungsindexdetektors und eines Lichtstreudetektors ermittelt. Die gelisteten Dispersitäten stammen aus der absoluten Messmethode.

Tabelle 5.1: Darstellung der gewichtsmittleren Molmassen der synthetisierten Poly(MA-HPBCHC) Proben. $\bar{M}_{w}$ (Poly(MMA)) bezeichnet das Ergebnis aus der Kalibrierung durch Poly(MMA). $\bar{M}_{w}$ (absolut) gibt das Ergebnis aus der Lichtstreudetektion an.

\begin{tabular}{cccc}
\hline Probe & $\begin{array}{c}\bar{M}_{w}(\text { Poly(MMA)) } \\
\mathrm{g} \mathrm{mol}^{-1}\end{array}$ & $\begin{array}{c}\bar{M}_{w} \text { (absolut) } \\
\mathrm{g} \mathrm{mol}^{-1}\end{array}$ & $\nexists$ \\
\hline M-1 & 73100 & 116710 & 1,15 \\
M-2 & 65160 & 76410 & 1,18 \\
M-3 & 31520 & 42620 & 1,21 \\
M-4 & 15880 & 23240 & 1,25 \\
M-5 & 17290 & 19450 & 1,21 \\
M-6 & 9130 & 11090 & 1,23 \\
\hline
\end{tabular}

Die Auftragung der gelisteten Messwerte nach (3.33) liefert $K=0,02967 \mathrm{~mL} \mathrm{~g}^{-1}$ und $a=0,566$. Die linearisierten Messwerte deuten auf eine Kettenlängenunabhängigkeit der Koeffizienten im untersuchten Molmassenbereich zwischen ca. $11000 \mathrm{~g} \mathrm{~mol}^{-1}$ und $110000 \mathrm{~g} \mathrm{~mol}^{-1}$. 


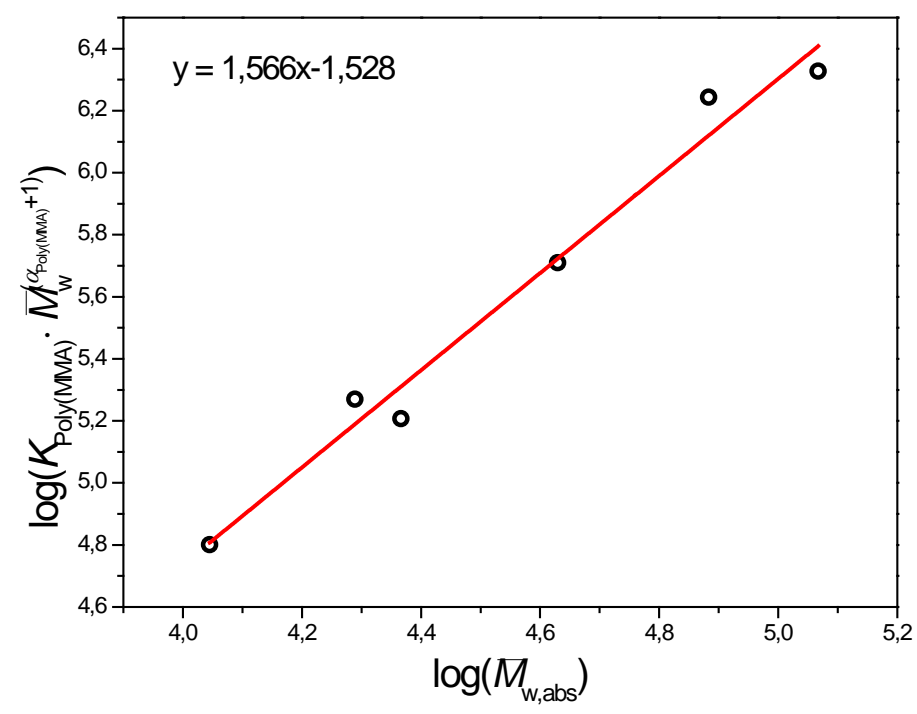

Abbildung 5.2: Bestimmung der Mark-Houwink-Parameter von Poly(MA-HPBCHC) durch Verwendung von SEC mit Poly(MMA)Kalibrierung und SEC gekoppelt mit Lichtstreuung von Polymerproben unterschiedlicher Molmassen.

\subsection{A-HPBCHC}

Die Herstellung der Polymerproben für A-HPBCHC (siehe Abbildung 5.3) erfolgte durch Polymerisation in Toluol (40 wt\%) und Zugabe von AiBN und 2-(Dodecylthiocarbonothioylthio)-2-methylpropionsäure (DDMAT). DDMAT ist in der Vergangenheit erfolgreich zur RAFT-Polymerisation von Acrylaten verwendet worden. ${ }^{[6]}$ Dabei wurden mit Ethylacrylat, Butylacrylat und 2-Hydroxyethylacrylat als Monomere, Polymere mit Dispersitäten unter 1,2 synthetisiert. ${ }^{[66]}$ Daher ist es zu erwarten, dass durch Zugabe dieses RAFT-Agenzien eine Kontrolle der Polymerisation von A-HPBCHC erreicht werden kann, da es sich dabei ebenfalls um ein Acrylat handelt. Zur Synthese wurde ein festes Verhältnis von 5:1 von RAFT-Agens zu AiBN verwendet. Die Polymerisationen wurden bis zu hohen Umsätzen $(X>0,8)$ durchgeführt. 
<smiles>C=CC(=O)OCCCCCCOC(=O)C1CCC(C2CCC(CCCCC)CC2)CC1</smiles>

\section{A-HPBCHC}

\section{Abbildung 5.3: Strukturformel von A-HPBCHC.}

In Tabelle 5.2 sind die gewichtsgemittelten Molmassen der synthetisierten Poly(A-HPBCHC) Proben aufgelistet die mittels SEC mit Poly(MMA) Kalibrierung und mit SEC und Lichtstreudetektion analysiert wurden. Die gelisteten Dispersitäten stammen aus der absoluten Messmethode. Variierende Molmassen konnten durch Änderung des Verhältnisses von Monomer zu RAFT-Agens erhalten werden. Wie zuvor in Abschnitt 5.2 wurde zur Bestimmung der absoluten Molmassen Lichtstreudetektion in der SEC verwendet und aufgrund des Produktverlustes während der Reinigung bleibt der Vergleich zwischen theoretischen und experimentellen Molmassen aus Gründen der Messgenauigkeit aus. Die synthetisierten Polymere zeigen geringe Dispersitäten unter 1,35 und eignen sich somit zur Bestimmung der Mark-Houwink-Koeffizienten mit der in Abschnitt 5.2 verwendeten Methode.

Tabelle 5.2: Darstellung der gewichtsmittleren Molmassen der synthetisierten Poly(A-HPBCHC) Proben. $\bar{M}_{w}$ (Poly(MMA)) bezeichnet das Ergebnis aus der Kalibrierung durch Poly(MMA), $\bar{M}_{w}$ (absolut) das Ergebnis aus der Lichtstreudetektion.

\begin{tabular}{cccc}
\hline Probe & $\begin{array}{c}\bar{M}_{w}\left(\text { Poly(MMA) }_{\text {g mol }^{-1}} /\right. \\
\text { A-1 }\end{array}$ & $\begin{array}{c}\bar{M}_{w} \text { (absolut) } / \\
\mathrm{g} \mathrm{mol}^{-1}\end{array}$ & $Ð$ \\
A-2 & 12190 & 10240 & 1,28 \\
A-3 & 28440 & 20330 & 1,25 \\
A-4 & 74400 & 42630 & 1,24 \\
A-5 & 69200 & 55880 & 1,31 \\
A-6 & 91480 & 71500 & 1,28 \\
\hline
\end{tabular}


Durch Auftragung der aufgelisteten Messwerte nach (3.33) liefert die Koeffizienten $K=0,01062 \mathrm{~mL} \mathrm{~g}^{-1}$ und $a=0,758$. Die Messwerte geben keinen Hinweis auf eine Kettenlängenabhängigkeit der Koeffizienten im untersuchten Molmassenbereich zwischen ca. $10000 \mathrm{~g} \mathrm{~mol}^{-1}$ und $90000 \mathrm{~g} \mathrm{~mol}^{-1}$.

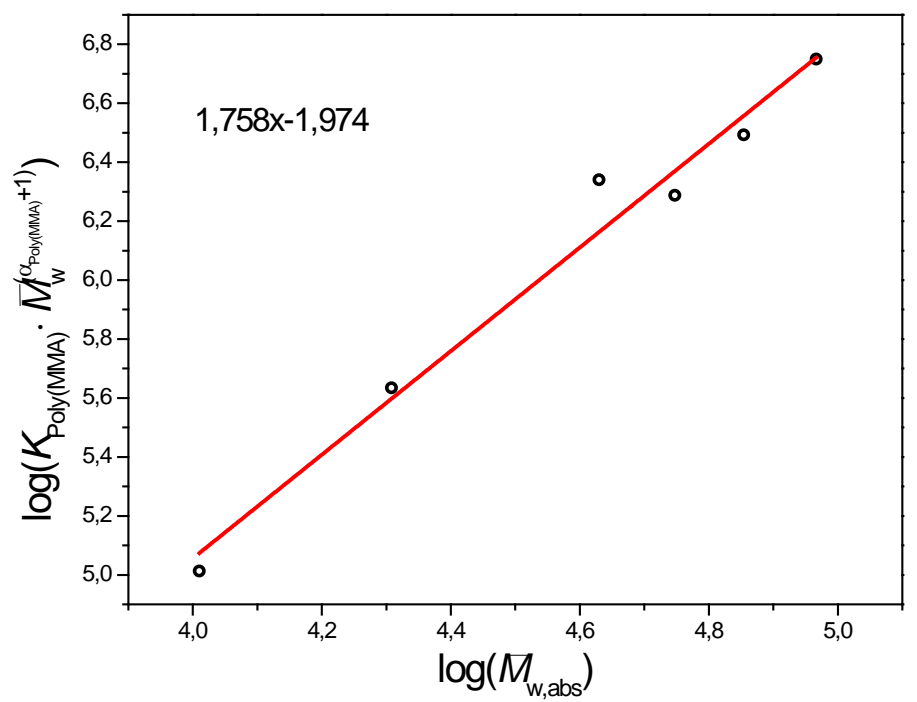

Abbildung 5.4: Bestimmung der Mark-Houwink-Parameter von Poly(A-HPBCHC) durch Verwendung von SEC mit Poly(MMA)-Kalibrierung und SEC gekoppelt mit Lichtstreuung von Polymerproben unterschiedlicher Molmassen.

\subsection{FA-HPBCHC}

Nach Kenntnis des Autors wurde zuvor nicht von der RAFTPolymerisation eines Fluoroacrylaten berichtet. Dementsprechend ist zur Synthese monodisperser Polymere durch diese Methode die Wahl des RAFT-Agens von großer Bedeutung. Hierzu wurde CDSTSP zur Kontrolle der Polymerisation von FA-HPBCHC ausgewählt. In Abschnitt 5.2 ist die Eignung von CDSTSP zur Kontrolle von RAFT-Polymerisationen erklärt worden. Die Polymerisation von FA-HPBCHC (siehe Abbildung 5.5) wurde in Toluol durch Zugabe von CDSTSP und AiBN im festen Verhältnis der letzteren von 2:1 durchgeführt. Das Verhältnis von 
Monomer zu CDSTSP wurde variiert, um Polymere verschiedener Kettenlängen zu erzeugen. Die synthetisierten Polymere sind in Tabelle 5.3 aufgelistet und wurden zur Bestimmung der Mark-Houwink-Koeffizienten verwendet.<smiles>C=C(F)C(=O)OCCCCCCOC(=O)C1CCC(C2CCC(CCCCC)CC2)CC1</smiles>

\section{FA-HPBCHC}

\section{Abbildung 5.5: Strukturformel von FA-HPBCHC.}

Eine Polymerisationsreihe wurde durchgeführt um die Kontrolle der Polymerisation durch das gewählte RAFT-Agens zu prüfen. Hierbei wurde die Reaktion in Toluol (20 wt\%) in einem Verhältnis von Monomer/CDSTSP/AiBN von 80:1:0,5 durchgeführt und bei unterschiedlichen Umsätzen abgebrochen. In Abbildung 5.6 sind die Ergebnisse der Polymerisationsreihe dargestellt. Zu beobachten ist, dass die Dispersitäten aller Polymerproben unter 1,5 liegen, vorwiegend bei ca. 1,3. Eine annähernd lineare Erhöhung der Molmasse mit steigendem Umsatz kann beobachtet werden. Dies sind Indizien dafür, dass eine Kontrolle der Reaktion stattfindet. Weiterhin wird für die Molmassenverteilungen eine Überlappung der Signale des RI- und des UV-Detektor (310 nm) beobachtet, wie in Abbildung 5.7 dargestellt. Daraus kann geschlossen werden, dass ein Großteil der Polymerketten RAFT-Endgruppen tragen, da FA-HPBCHC selbst bei dieser Wellenlänge nicht absorbiert. Entgegen der Erwartung ist die nahezu konstant bleibende Dispersität. Für eine reversibel desaktivierte radikalische Polymerisation wird eine Abnahme mit steigendem Umsatz erwartet. 


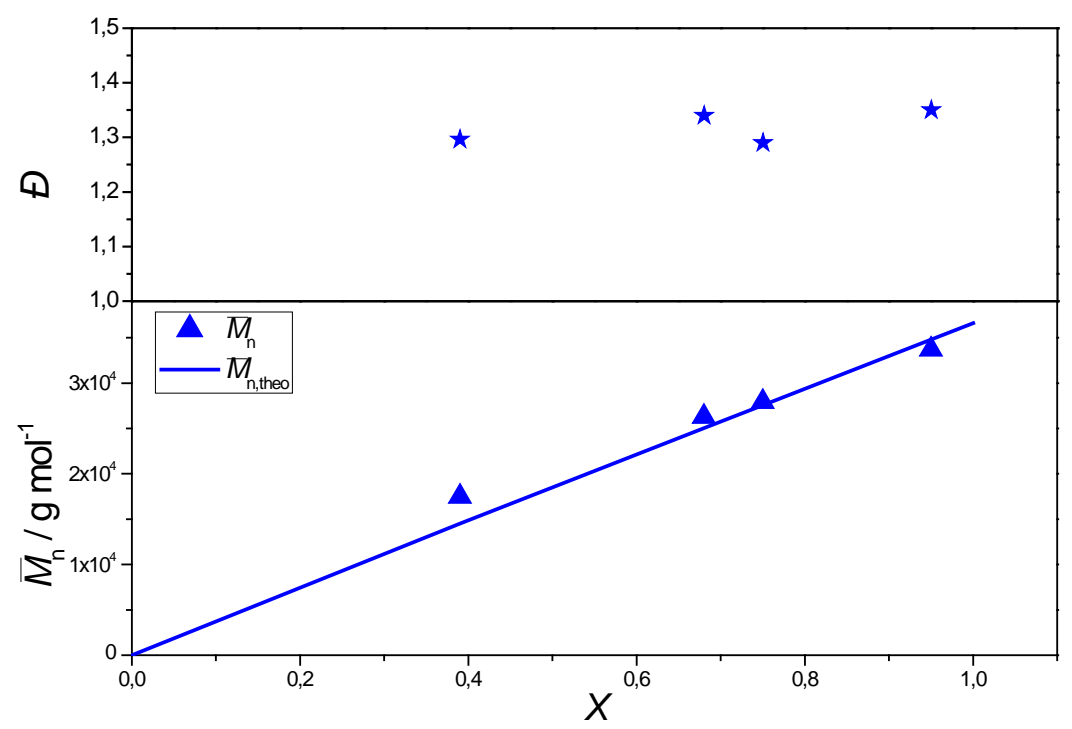

Abbildung 5.6: Dargestellt sind die Ergebnisse der Polymerisationsreihe von FA-HPBCHC. Im unteren Graph ist die Entwicklung von $\bar{M}_{n}$ nach dem Umsatz abgebildet und im oberen die Dispersitäten der Polymerprodukte. Die Methode der universellen Kalibrierung wurde angewendet. Hierzu wurden die in diesem Abschnitt ermittelten Mark-Houwink-Koeffizienten verwendet.

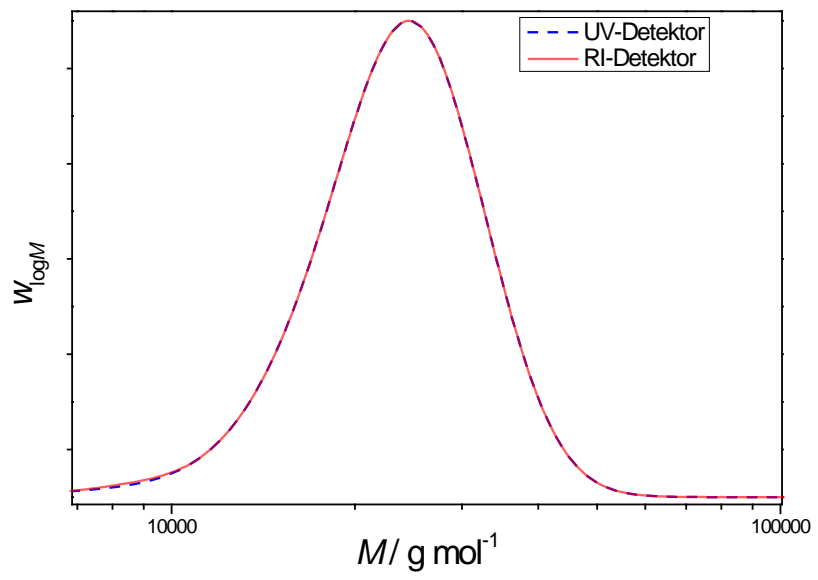

Abbildung 5.7: Molmassenverteilung durch SEC gemessen eines FA-HPBCHC Polymers synthetisiert durch RAFT-Polymerisation mit CDSTSP. In Rot ist das Signal des Brechungsindexdetektors dargestellt und in Blau das des UV-Detektors (310 nm). 
Die Analyse der Polymerproben von FA-HPBCHC mittels SEC gekoppelt mit Lichtstreudetektion ergab ein nicht ausreichendes Signal-RauschVerhältnis. In Abbildung 5.8 ist das Signal des Brechungsindexdetektors und des Lichtstreudetektors für eine SEC Messung einer Poly(FA-HPBCHC) Probe dargestellt. Hierbei wurde eine ungewöhnlich hohe Konzentration an Polymer verwendet (25 $\left.\mathrm{mg} \mathrm{mL}^{-1}\right)$, um das SignalRausch-Verhältnis zu verbessern.

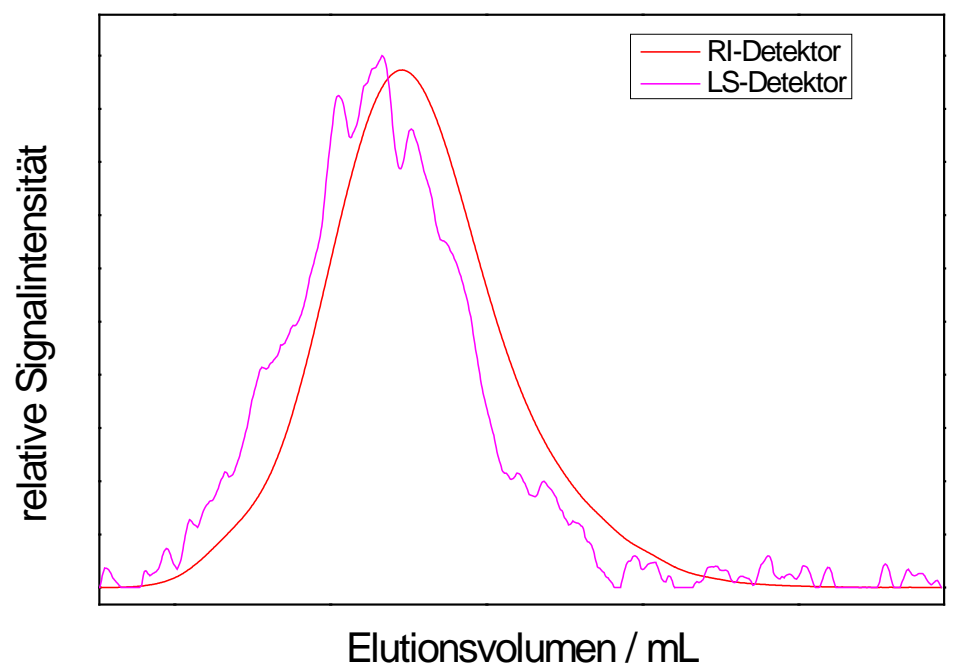

Abbildung 5.8: Molmassenverteilung durch SEC gemessen eines FA-HPBCHC Polymers synthetisiert durch RAFT-Polymerisation mit CDSTSP. In Rot ist das Signal des Brechungsindexdetektors dargestellt und in Magenta das des Lichtstreudetektors.

$\mathrm{Zu}$ beobachten ist, dass während der Brechungsindexdetektor ein ausreichendes Signal-Rausch-Verhältnis vorweist, der Lichtstreudetektor wegen der geringen Signalintensität eine unzureichende Beschreibung des Verlaufs der Molmassenverteilung liefert. Der Grund für dieses Phänomen liegt vermutlich in einem sehr geringen Brechungsindexinkrement $d n / d c$. Dies lässt sich durch das Vorhandensein sehr ähnlicher Brechungsindices von Polymer und Lösungsmittel erklären. Fluoroacrylate zeigen im allgemeinen niedrige Brechungsindices. ${ }^{[67,68]}$ Es ist zu erwarten, dass dies auch für FA-HPBCHC zutrifft und somit der Brechungsindex nahe dem des Eluenten THF liegt. Der Grund für die höhere Sensitivität des 
Brechungsindexdetektors im Vergleich zum Lichtstreudetektor liegt in der unterschiedlichen Abhängigkeit der Signalintensität zum Brechungsindexinkrement.

$$
\begin{gathered}
\text { Signalintensität }(\mathrm{RI})=K_{\mathrm{RI}} \cdot \frac{d n}{d c} \cdot c_{\mathrm{P}} \\
\text { Signalintensität }(\mathrm{LS})=K_{\mathrm{LS}} \cdot\left(\frac{d n}{d c}\right)^{2} \cdot c_{\mathrm{P}} \cdot \bar{M}_{w}
\end{gathered}
$$

Mit $K_{\mathrm{BI}}$ und $K_{\mathrm{LS}}$ als Proportionalitätskonstanten des jeweiligen Detektors und $c_{\mathrm{P}}$ als Polymerkonzentration. Daraus resultierend wurde Lichtstreudetektion als ungeeignet zur Bestimmung von absoluten Molmassen von Poly(FA-HPBCHC) eingestuft.

Ein alternatives Verfahren zur Bestimmung der absoluten Molmasse wurde entwickelt. Dazu wurde die Tatsache ausgenutzt, dass CDSTSP, wie viele andere RAFT-Agenzien, im UV-Bereich Strahlung absorbiert. Schon in der Vergangenheit ist darauf hingewiesen worden, dass dies zur quantitativen Bestimmung der Endgruppen verwendet werden könnte und somit zur Bestimmung der zahlenmittleren Molmasse. ${ }^{[69]}$ Im Rahmen dieser Arbeit wurde dieser Gedanke angewendet indem SEC mit Brechungsindex- und UV-Detektion gekoppelt wurde. Die Brechungsindexdetektion wurde, wie schon zuvor in den Abschnitten 5.2 und 5.3, zur Bestimmung der Referenzwerte durch Poly(MMA)-Kalibrierung verwendet. Die UVDetektion dagegen ergab nach einem Kalibrierungsvorgang mit dem reinen RAFT-Agens die zahlenmittlere Molmasse durch Integration des Signals über das Elutionsvolumen. Im Folgenden soll die Methode detailliert geschildert werden.

$\mathrm{Zu}$ Beginn wurden Stammlösungen von CDSTSP in THF (Elutionsmittel der SEC) in einem Konzentrationsbereich von $40 \mu \mathrm{g} \mathrm{mL}^{-1}$ bis $160 \mu \mathrm{g} \mathrm{mL}^{-1}$ hergestellt. Diese Proben wurden durch SEC mit UV-Detektion vermessen und das Integral der Signalintensität gegen das Elutionsvolumen berechnet. Als Detektionswellenlänge wurde $310 \mathrm{~nm}$ ausgewählt. Somit konnte durch Auftragung von Konzentration der Stammlösung gegen das Integral des 
Detektorsignals des gesamten Probenvolumens ( $\int \mathrm{DS}(\mathrm{UV})$ ) eine Kalibrierkonstante $k_{\mathrm{UV}}$ aus der Steigung gewonnen werden.

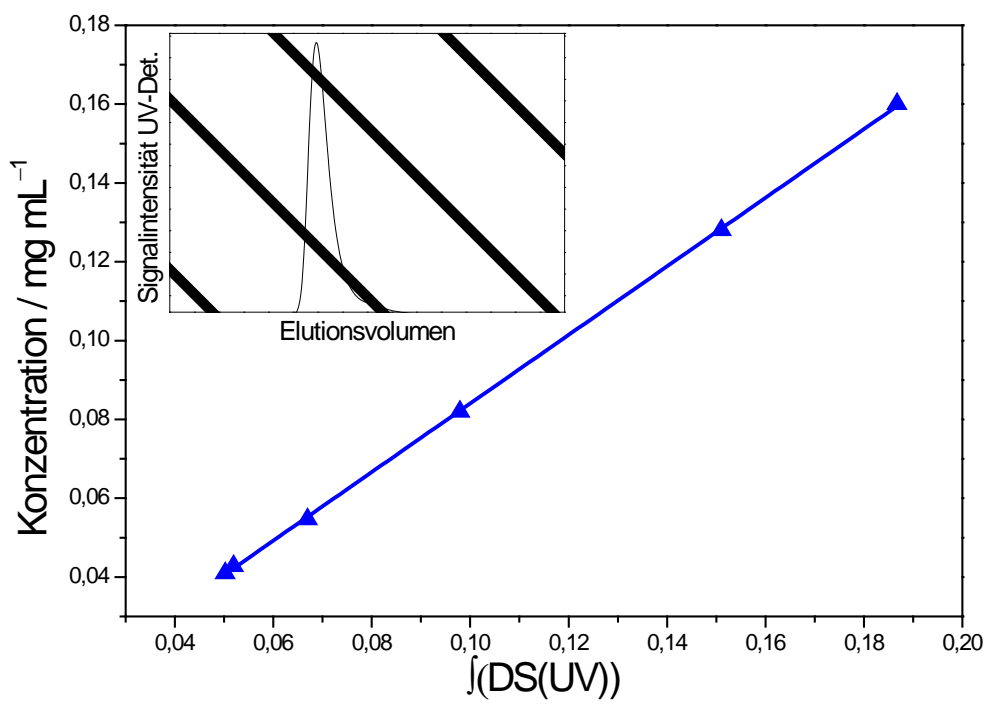

Abbildung 5.9: Bestimmung der Kalibrierkonstanten $k_{\mathrm{UV}}$ des SEC UVDetektors für CDSTSP durch Linearisierung des Integrals des Detektorsignals von CDSTSP-Stammlösungen. In der linken oberen Ecke ist ein Elugramm von CDSTSP aufgenommen durch UV-Detektion dargestellt. Die Detektionswellenlänge ist $310 \mathrm{~nm}$.

Das hier zu untersuchende Monomer FA-HPBCHC zeigte keine Absorption bei $310 \mathrm{~nm}$ in dem verwendeten Konzentrationsbereich. Dementsprechend konnte durch die gleiche Vorgehensweise die zahlenmittlere Molmasse der synthetisierten RAFT-Polymere ermittelt werden. Hierzu muss angenommen werden, dass jede Polymerkette eine RAFT-Endgruppe besitzt. Durch Kopplung mit der zuvor bestimmten Kalibrierkonstante kann die Konzentration an RAFT-Gruppen in der Polymerprobe bestimmt und aus der Einwaage das Verhältnis von Monomereinheiten zu RAFTGruppen bestimmt werden. Zusammengefasst lässt sich folgende Gleichung aufstellen. 


$$
\bar{M}_{n}=\frac{\left(c_{\mathrm{P}}-\int \mathrm{DS}(\mathrm{UV}) \cdot k_{\mathrm{UV}}\right) \cdot M_{\mathrm{CDSTSP}}}{\int \mathrm{DS}(\mathrm{UV}) \cdot k_{\mathrm{UV}}}+M_{\mathrm{CDSTSP}}
$$

Wie zuvor in Abschnitt 5.2 erklärt, wird zur Bestimmung der MarkHouwink-Koeffizienten $\bar{M}_{w}$ benötigt. Dies wird durch den grundlegenden Zusammenhang (5.4) errechnet.

$$
\bar{M}_{w}=\bar{M}_{n} \cdot \oslash
$$

Aus der Analyse der synthetisierten Polymere wurde der in Tabelle 5.3 aufgelistete Datensatz erhalten. Dargestellt sind die aus der SEC mit Poly(MMA)-Kalibrierung und aus der UV-Detektion gewonnenen gewichtsgemittelten Molmassen. Die aufgetragenen Dispersitäten wurden aus der relativen Messmethode entnommen.

Tabelle 5.3: Darstellung der gewichtsmittleren Molmassen der synthetisierten Poly(FA-HPBCHC) Proben. $\bar{M}_{w}$ (Poly(MMA)) bezeichnet das Ergebnis aus der Kalibrierung durch Poly(MMA), während $\bar{M}_{w}$ (absolut) das Ergebnis aus der zuvor beschriebenen UV-Detektionsmethode kennzeichnet.

\begin{tabular}{cccc}
\hline Probe & $\begin{array}{c}\bar{M}_{w}\left(\mathrm{Poly}_{\mathrm{MMA})}\right) / \\
\mathrm{g} \mathrm{mol}^{-1}\end{array}$ & $\begin{array}{c}\bar{M}_{w} \text { (absolut) } \\
\mathrm{g} \mathrm{mol}^{-1}\end{array}$ & $Ð$ \\
\hline FA-1 & 5710 & 12680 & 1,31 \\
FA-2 & 7990 & 17490 & 1,22 \\
FA-3 & 11770 & 28880 & 1,24 \\
FA-4 & 16100 & 35780 & 1,20 \\
FA-5 & 21880 & 45680 & 1,16 \\
FA-6 & 36520 & 77770 & 1,40 \\
FA-7 & 39350 & 87440 & 1,38 \\
FA-8 & 46250 & 102770 & 1,41 \\
\hline
\end{tabular}


Die Auftragung der aufgelisteten Messwerte nach (3.33) liefert $K=0,00260 \mathrm{~mL} \mathrm{~g}^{-1}$ und $a=0,714$. Die linearisierten Messwerte deuten auf eine Kettenlängenunabhängigkeit der Koeffizienten im untersuchten Molmassenbereich zwischen ca. $10000 \mathrm{~g} \mathrm{~mol}^{-1}$ und $100000 \mathrm{~g} \mathrm{~mol}^{-1}$.

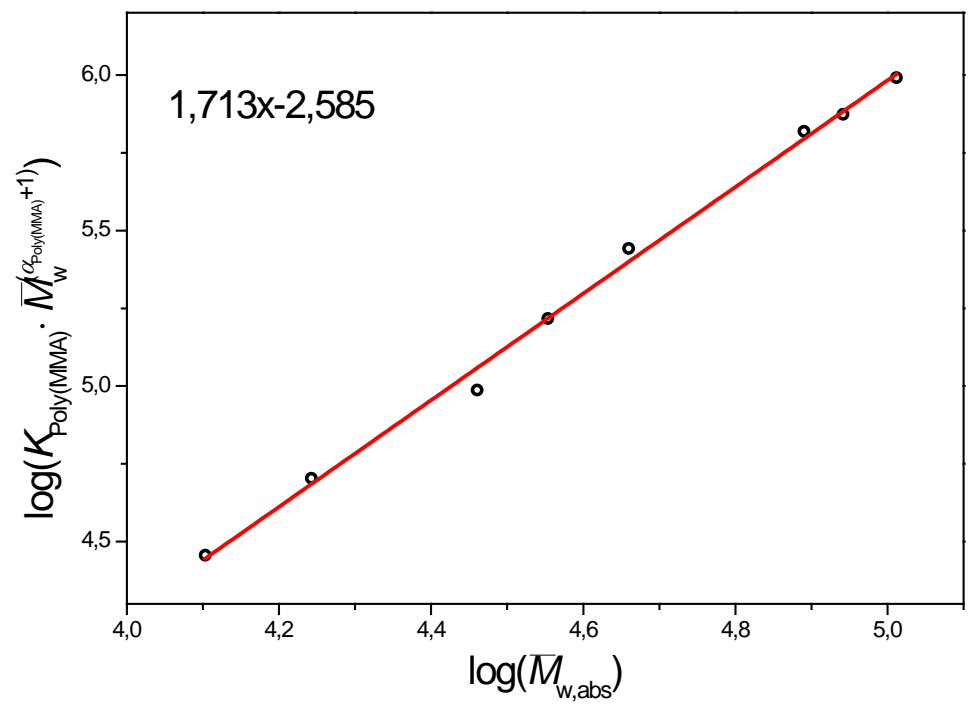

Abbildung 5.10: Bestimmung der Mark-Houwink-Parameter von Poly(FA-HPBCHC) durch Verwendung von SEC mit Poly(MMA) Kalibrierung und SEC gekoppelt mit UV-Detektion von Polymerproben unterschiedlicher Molmassen.

In diesem Kapitel wurde die Bestimmung der Mark-HouwinkKoeffizienten von Poly(MA-HPBCHC), Poly(A-HPBCHC) und Poly(FA-HPBCHC) vorgestellt. Die gewonnenen Parameter werden in den folgenden Kapiteln zur Berechnung absoluter Molmassenverteilungen aus SEC verwendet. 


\section{Propagation von MA-HPBCHC}

\subsection{Einleitung}

In diesem Kapitel wird die Messung des Propagationskoeffizienten $k_{\mathrm{p}}$ für das reaktive Mesogen MA-HPBCHC mittels PLP-SEC beschrieben. Weiterhin werden die Ergebnisse im Kontext anderer Methacrylate eingeordnet. Aufgrund des hohen Schmelzpunktes von MA-HPBCHC können die Experimente nicht in Substanz durchgeführt werden, sodass Toluol als Lösungsmittel ausgewählt wurde. Toluol eignet sich als Lösungsmittel für diese Grundsatzuntersuchungen, denn es besitzt niedrige Transferraten und weicht in seiner Polarität nicht stark vom Monomer und den in Kapitel 10 untersuchten nematischen und chiral-nematischen Phasen $a b .{ }^{[70]}$ Grundsätzlich werden Propagationskoeffizienten nur sehr geringfügig durch Lösungsmittel beeinflusst, die eine ähnliche Polarität besitzen. ${ }^{[71]}$ Anschließend werden die Ergebnisse in Toluol weiteren PLP-SECExperimenten in der nematischen Flüssigkeit BL-087 (Merck KGaA) gegenübergestellt. Die Analyse der Polymer-Produkte erfolgte durch SEC mit Brechungsindexdetektion. Die Molmassenverteilungen wurden nach dem Prinzip der universellen Kalibrierung mithilfe der in Abschnitt 5.2 bestimmten Mark-Houwink-Koeffizienten in absolute Verteilungen umgerechnet.

\subsection{Strukturanalyse der PLP-SEC Kurven}

Die Experimente wurden mit einem Anteil von 20 wt\% MA-HPBCHC in Toluol durchgeführt. Jede Probe wurde zweimal per SEC analysiert, um ein 
Maß für die Abweichung durch die SEC-Analyse zu ermitteln. Gemäß der IUPAC-Richtlinien wurden die Messwerte durch Variation der Pulsfrequenz und Initiatorkonzentration verifiziert. ${ }^{[54]}$ Die PLPExperimente wurden in einem Temperaturbereich zwischen 20 und $80{ }^{\circ} \mathrm{C}$ durchgeführt. Die Pulsanzahl wurde derart eingestellt, dass ein maximaler Umsatz von $5 \%$ bewerkstelligt wurde. Somit konnte einerseits genug Polymermaterial zur SEC Analyse erhalten werden und andererseits von einer konstanten Monomerkonzentration ausgegangen werden.

Drei durch PLP erhaltene SEC-Kurven, aufgenommen bei $60{ }^{\circ} \mathrm{C}$, sind in Abbildung 6.1 dargestellt. Hierbei wurde bei A eine Pulsfrequenz $\left(v_{\text {rep }}\right)$ von $5 \mathrm{~Hz}$, bei B eine Pulsfrequenz von $16 \mathrm{~Hz}$ und bei $\mathbf{C}$ eine Pulsfrequenz von $30 \mathrm{~Hz}$ angewendet.
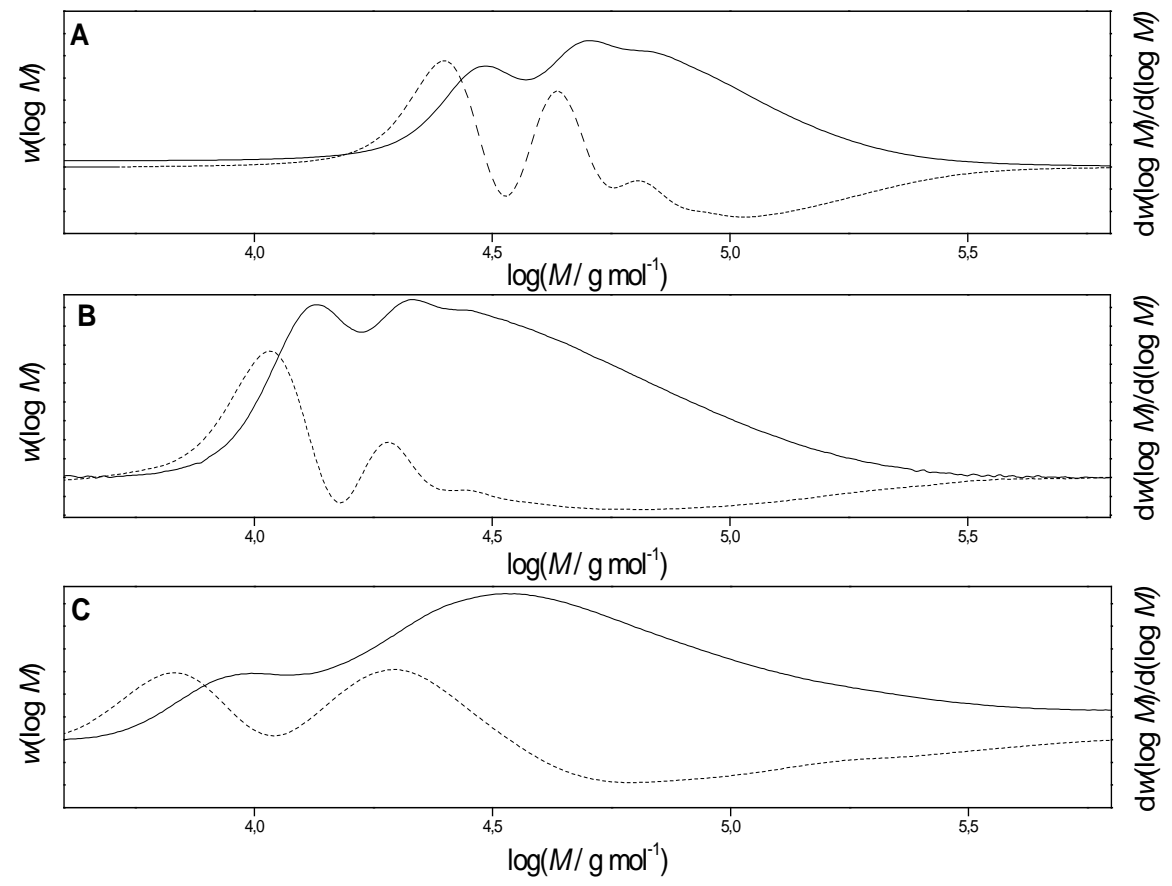

Abbildung 6.1: Molmassenverteilung (durchgezogene Linie) gewonnen aus SEC für Poly(MA-HPBCHC) hergestellt durch PLP $\left(60^{\circ} \mathrm{C}, 20\right.$ wt $\%$ in Toluol, 50 mmol DMPA) und erste Ableitung (gepunktete Linie). Graph A wurde mit $v_{\text {rep }}=5 \mathrm{~Hz}$, Graph B mit $v_{\text {rep }}=16 \mathrm{~Hz}$ und Graph C mit $v_{\text {rep }}=30 \mathrm{~Hz}$ aufgenommen. 
$\mathrm{Zu}$ sehen in der ersten Ableitung sind ausgeprägte Maxima, die die Wendepunkte (eng: point of inflection, POI) in der Molmassenverteilung kennzeichnen. Aus der Literatur ist bekannt dass sich die POIs zur Beschreibung der kinetischen Kettenlänge der Polymere eignen, dessen Lebenszeit der Zeitraum zwischen zwei Laserpulsen entspricht, wie in Abschnitt 3.6 erläutert. Dementsprechend kann $k_{\mathrm{p}}$ aus Gleichung (3.30) bestimmt werden.

Wie aus der Abbildung ersichtlich variiert die Struktur der erhaltenen SECKurven stark mit angewandter Pulsfrequenz. Hierbei wird ersichtlich, dass dieses System empfindlich gegenüber Variationen von $v_{\text {rep }}$ ist. $\mathrm{Zu}$ beobachten ist, dass A und B Strukturen zeigen welche dem ITC (eng: intermediate-termination case) entsprechen ${ }^{[72]}$ In diesem Fall geben die POIs akkurate Beschreibungen von $k_{\mathrm{p}} \cdot{ }^{[72]}$ Im Idealfall erreicht der Koeffizient $M_{1} / M_{2}$ den Wert 0,5. Je höher $v_{\text {rep }}$ desto stärker verschieben sich die Strukturen in Richtung LTRL (eng: low-termination-rate-limit). ${ }^{\text {[72] }}$ Im Falle des Graphen $\mathbf{C}$ ist der Grenzfall überschritten. Die gezeigte Struktur wird nicht mehr durch den PLP-Prozess kontrolliert. Der Koeffizient $M_{1} / M_{2}$ beträgt in dieser Messung 0,34. Als Schlussfolgerung muss gelten, dass ein eingeschränkter Pulsfrequenzbereich im vorliegenden System zur Bestimmung von $k_{\mathrm{p}}$ verwendet werden kann. Aus diesem Grund wurde die Pulsfrequenzabhängigkeit von $k_{\mathrm{p}}$ untersucht. In Abbildung 6.2 sind die Ergebnisse von PLP-SEC Experimente bei $60^{\circ} \mathrm{C}$ und einer Initiatorkonzentration von $50 \mathrm{mmol}$ gegen $v_{\text {rep }}$ aufgetragen. 


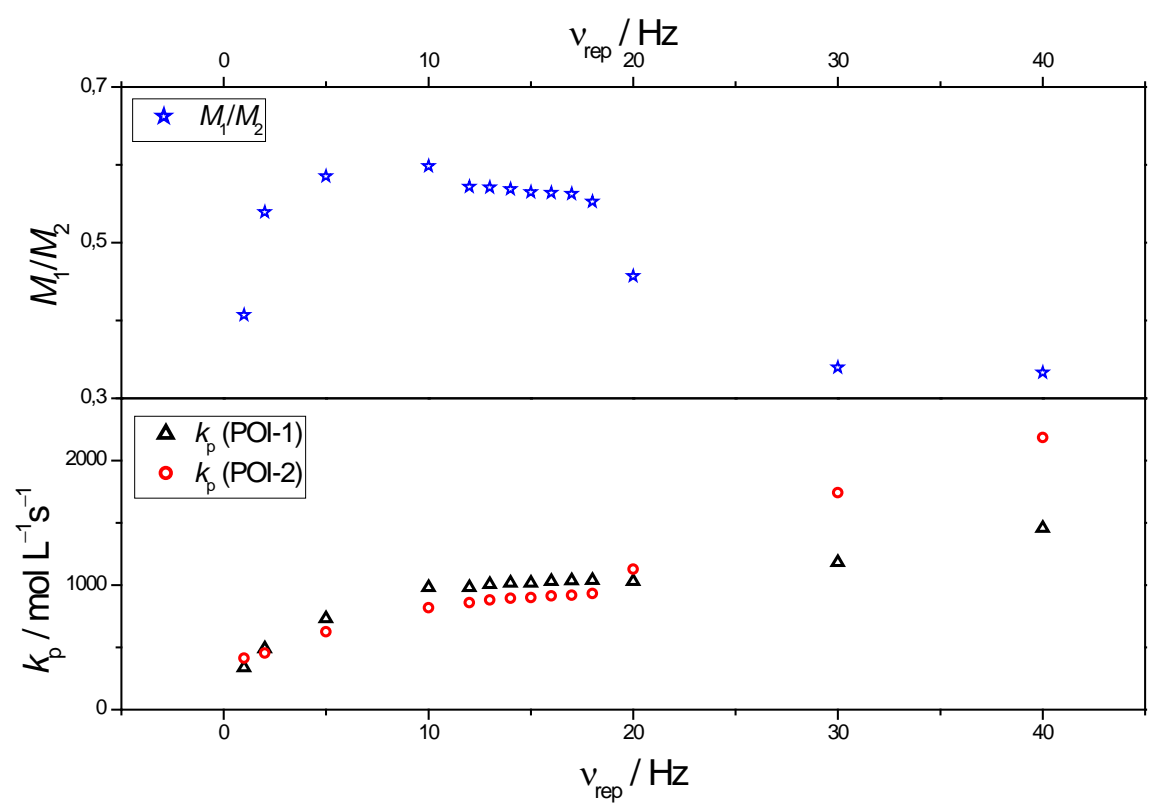

Abbildung 6.2: Ergebnisse der pulsfrequenzabhängigen PLP-SECExperimente für MA-HPBCHC in Toluol bei $60^{\circ} \mathrm{C}(20 \mathrm{wt} \%, 50 \mathrm{mmol}$ DMPA). Im unteren Bereich sind die aus dem ersten Wendepunkt ( $k_{\mathrm{p}}(\mathrm{POI}-1)$ ) und dem zweiten Wendepunkt ( $\left.\boldsymbol{k}_{\mathrm{p}}(\mathrm{POI}-2)\right)$ erhaltenen $\boldsymbol{k}_{\mathrm{p}}$ Werte aufgetragen. Im oberen Bereich sind die entsprechenden Verhältnisse der Molmassen $M_{1} / M_{2}$ gezeigt.

$\mathrm{Zu}$ beobachten ist die zuvor erwähnte Pulsfrequenzabhängigkeit der Ergebnisse. Bei sehr niedrigen $v_{\text {rep }}$ zeigen die $k_{\mathrm{p}}$ Werte keine Konsistenz zu anderen Pulsfrequenzen und der Quotient $M_{1} / M_{2}$ zeigt eine hohe Abweichung vom Idealwert 0,5. Diese Strukturen liegen abseits des HTRL (eng: high-termination-rate-limit). ${ }^{[72]}$ In diesem Fall ist der Anteil der Radikalpopulation dessen Lebenszeit durch Laserpulseinstrahlung kontrolliert wird geringfügig. Ein prädominierender Anteil wird in der Totzeit zwischen den Laserpulsen terminiert. Dies setzt einen hohen Terminierungskoeffizienten voraus, welcher im vorliegenden System einerseits aufgrund der niedrigen Viskosität von Toluol und andererseits aufgrund der geringen Monomerkonzentration und der daraus resultierenden kurzen Makroradikalketten (Kettenlängenabhängigkeit von $k_{\mathrm{t}}$, siehe Abschnitt 3.4.2) resultiert. Ab einer $v_{\text {rep }}$ von $10 \mathrm{~Hz}$ wird ein Plateaubereich erreicht. Dort zeigen die Werte eine nur sehr geringe 
Abhängigkeit des $k_{\mathrm{p}}$ von $v_{\text {rep }}$ die innerhalb der Messgenauigkeit von PLPSEC-Messungen liegt. Dementsprechend wird die IUPAC Empfehlung für Datenkonsistenz in diesem Bereich durch Reproduzierbarkeit bei Variation von $v_{\text {rep }}$ eingehalten. Der Koeffizient $M_{1} / M_{2}$ nähert sich dem Idealwert von 0,5 an ohne ihn jedoch zu erreichen. Ab $20 \mathrm{~Hz}$ sinkt $M_{1} / M_{2}$ unter 0,5 , der LTRL wird überschritten. In diesem Falle ist das Verhältnis von Wachstumsdauer zu Terminierungsdauer nicht ausreichend, um gültige PLP Strukturen auszubilden.

Zur Bestimmung der Propagationskoeffizienten wurde für jede Temperatur die $v_{\text {rep }}$-Abhängigkeit untersucht. Anschließend wurden die Bereiche in dem $k_{\mathrm{p}}$ bei verschiedenen $v_{\text {rep }}$ reproduziert werden konnte ausgewählt und die $k_{\mathrm{p}}$ Werte durch Einsatz von verschiedenen Initiatorkonzentrationen validiert. Alle Messdaten die zur Berechnung von $k_{\mathrm{p}}$ verwendet wurden sind in Abschnitt 11 aufgelistet.

\subsection{Temperaturabhängigkeit von $k_{\mathrm{p}}$}

Zur Bestimmung der Aktivierungsenergie $E_{\mathrm{A}}$ und des präexponentiellen Faktors $A$ des Propagationskoeffizienten von MA-HPBCHC wurden PLPSEC Messungen in einem Temperaturbereich von $20^{\circ} \mathrm{C}$ bis $80^{\circ} \mathrm{C}$ durchgeführt. Die Auftragung der Messwerte nach Arrhenius liefert durch lineare Anpassung $E_{\mathrm{A}}$ und $A$, wie in Abbildung 6.3 dargestellt. 


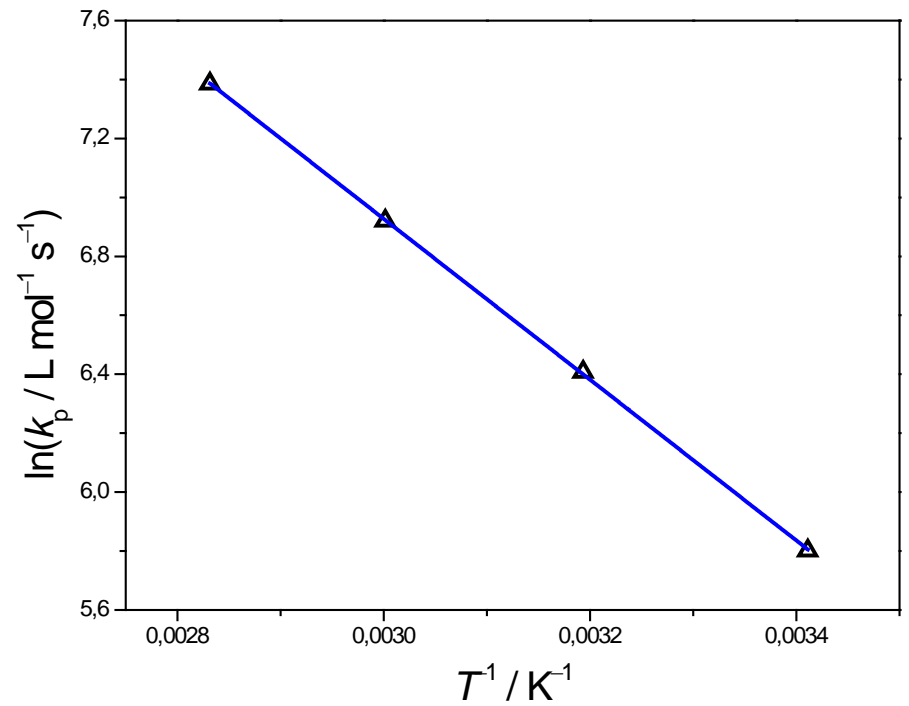

Abbildung 6.3: Arrheniusplot der Propagationskoeffizienten von МА-НРВСНС.

Für die Temperaturabhängigkeit der Propagationskoeffizienten von MA-HPBCHC wurde folgender Zusammenhang ermittelt.

$$
\ln \left(k_{\mathrm{p}} / \mathrm{L} \mathrm{mol}^{-1} \mathrm{~s}^{-1}\right)=15,12-2729 /(T / \mathrm{K})
$$

Hierbei kennzeichnet $T$ die absolute Temperatur. Aus (6.1) ergib sich eine Aktivierungsenergie $E_{\mathrm{A}}=22,7 \mathrm{~kJ} \mathrm{~mol}^{-1}$ und $A=3,69 \cdot 10^{6} \mathrm{~L} \mathrm{~mol}^{-1} \mathrm{~s}^{-1}$. Der Fehler der Messungen kann mittels eines $95 \%$ Konfidenzintervalls ermittelt werden. Diese Abschätzung wurde durch die Annahme eines konstanten relativen Fehlers und der Anpassung durch die Methode der kleinsten Quadrate durchgeführt, wie von van Herk vorgeschlagen. ${ }^{[73]}$ Das Ergebnis dieser Analyse ist in Abbildung 6.4 dargestellt. 


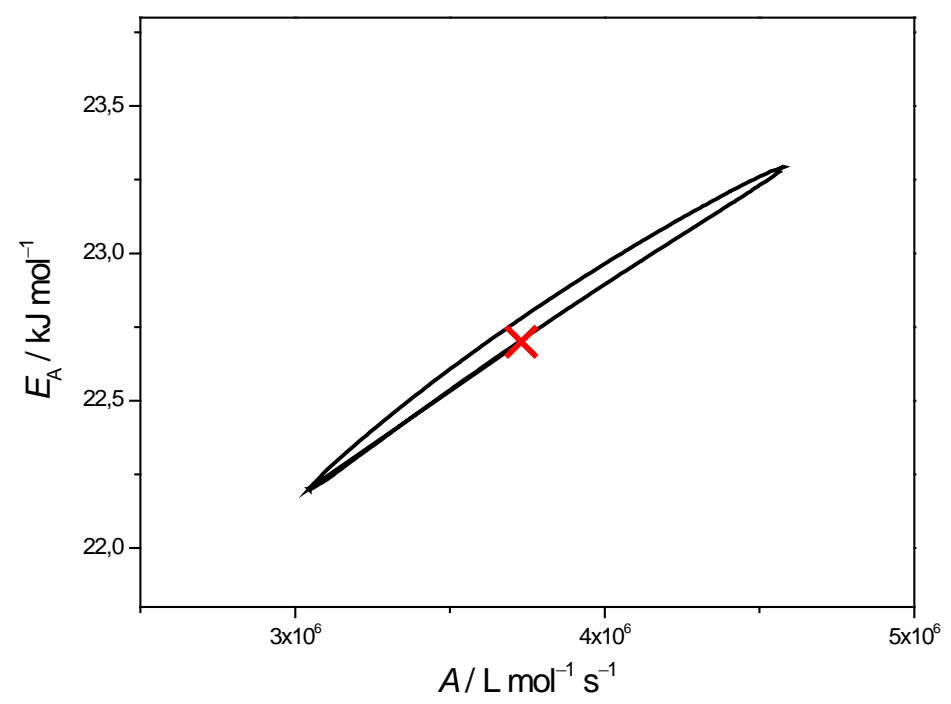

Abbildung 6.4: 95 \% Konfidenzintervall Region der Arrhenius Parameter des Propagationskoeffizienten von MA-HPBCHC. Das rote Kreuz gibt das Wertepaar mit der höchsten Wahrscheinlichkeit an.

Es ergibt sich für die Arrhenius Parameter folgende Messungenauigkeit $22,2 \mathrm{~kJ} \mathrm{~mol}^{-1}<E_{\mathrm{A}}<23,3 \mathrm{~kJ} \mathrm{~mol}^{-1}$

und $3,1 \cdot 10^{6} \mathrm{~L} \mathrm{~mol}^{-1} \mathrm{~s}^{-1}<A<4,6 \cdot 10^{6} \mathrm{~L} \mathrm{~mol}^{-1} \mathrm{~s}^{-1}$. Das Intervall des Fehlers ergibt sich vorwiegend aus der Streuung der Messwerte, während eine Variation des relativen Fehlers sehr ähnliche Verläufe lieferte. Die erhaltenen Fehlerbereiche sind gut vergleichbar mit denen von Datensätze anderer Methacrylate. ${ }^{[74]}$

In Tabelle 6.1 sind die Aktivierungsenergien, präexponentielle Faktoren und Propagationskoeffizienten bei $50^{\circ} \mathrm{C}$ von verschiedenen Methacrylaten aufgelistet. Zu beobachten ist innerhalb von $E_{\mathrm{A}}$ Abweichung von ca. $3 \mathrm{~kJ}$ $\mathrm{mol}^{-1}$ und innerhalb von A Abweichungen von $6 \cdot 10^{6} \mathrm{~L} \mathrm{~mol}^{-1} \mathrm{~s}^{-1}$. Bezogen auf die ermittelte Messunsicherheit von MA-HPBCHC und der Literaturwerte ist ein Ableitung von Familienverhalten und Trends in Bezug auf die einzelnen Arrhenius-Parameter nicht möglich. Beim Vergleich der $k_{\mathrm{p}}$-Werte bei gegebenen Temperaturen können jedoch einige Abhängigkeiten beobachtet werden. 
Tabelle 6.1: Auflistung der Aktivierungsenergien, präexponentielle Faktoren und Propagationskoeffizienten bei $50^{\circ} \mathrm{C}$ von verschiedenen Methacrylaten. Die Monomere sind unterteilt in lineare und verzweigte Seitenketten. Innerhalb dieser Kategorien sind die Substanzen nach abgeschätzter steigender sterischer Hinderung der Seitenkette geordnet.

\begin{tabular}{|c|c|c|c|c|}
\hline & Seitenkette & $\begin{array}{l}E_{\mathrm{A}} \\
\mathrm{kJ} \mathrm{mol}^{-1}\end{array}$ & $\begin{array}{l}A \\
\mathrm{~L} \mathrm{~mol}^{-1} \mathrm{~s}^{-1}\end{array}$ & $\begin{array}{l}k_{\mathrm{p}} \text { bei } 50{ }^{\circ} \mathrm{C} / \\
\mathrm{L} \mathrm{mol}^{-1} \mathrm{~s}^{-1}\end{array}$ \\
\hline \multirow{4}{*}{ Linear } & Methyl $^{[6]}$ & 22,4 & $2,67 \cdot 10^{6}$ & 650 \\
\hline & Butyl $^{[6]}$ & 22,9 & $3,78 \cdot 10^{6}$ & 760 \\
\hline & Dodecyl $^{[6]}$ & 21,0 & $2,50 \cdot 10^{6}$ & 1000 \\
\hline & Behenyl $^{[74]}$ & 20,5 & $2,51 \cdot 10^{6}$ & 1170 \\
\hline \multirow{9}{*}{ verzweigt } & tert-Butyl $^{[75]}$ & 22,1 & $2,40 \cdot 10^{6}$ & 640 \\
\hline & Cyclohexyl $^{[76]}$ & 23,7 & $7,90 \cdot 10^{6}$ & 1170 \\
\hline & Benzyl $^{[76]}$ & 20,3 & $2,60 \cdot 10^{6}$ & 1360 \\
\hline & Glycidyl $^{[76]}$ & 23,2 & $6,70 \cdot 10^{6}$ & 1190 \\
\hline & Isobornyl $^{[77]}$ & 22,5 & $4,28 \cdot 10^{6}$ & 1002 \\
\hline & Ethylhexyl $^{[78]}$ & 20,4 & $1,87 \cdot 10^{6}$ & 944 \\
\hline & Propylheptyl $^{[74]}$ & 21,7 & $2,83 \cdot 10^{6}$ & 880 \\
\hline & Heptadecany $^{[74]}$ & 20,7 & $2,04 \cdot 10^{6}$ & 920 \\
\hline & НРВСНС & 22,7 & $3,69 \cdot 10^{6}$ & 790 \\
\hline
\end{tabular}

Im Falle der linearen Methacrylate ist ein Anstieg der Propagationskoeffizienten mit steigender Länge der Seitenkette zu beobachten. Als Erklärung für diesen Anstieg wurde eine Strukturierung der Lösung mit der Folge einer erhöhten lokalen Monomerkonzentration um das Radikalzentrum vorgeschlagen. ${ }^{[74]}$ Dagegen sprechen PLP-SEC Experimente in Lösung, welche keine Anhängigkeit der $k_{\mathrm{p}}$ Werte vom Lösungsmittelanteil ergaben. ${ }^{[74]}$ Eine weitere Erklärung ist die Beeinflussung der Rotationsbarriere im Übergangszustand durch Abschirmung der Estergruppen durch die verlängerte Alkylreste. ${ }^{[79]}$ Hierbei 
wäre vorwiegend ein Einfluss auf den präexponentiellen Faktor und eine Konstanz der Aktivierungsenergie zu erwarten. Dies in den experimentellen Ergebnissen nicht zu beobachten. Somit scheint bis zum jetzigen Zeitpunkt keine zufriedenstellende Antwort auf das Verhalten linearer Methacrylate vorzuliegen.

Im Falle der verzweigten Methacrylaten erscheint die Interpretation der momentanen Sachlage weniger eindeutig. Klare Korrelationen zwischen Struktur und Propagationsgeschwindigkeit sind nicht zu erkennen. Eine Erhöhung von $k_{\mathrm{p}}$ mit größer werdender Seitenkette ist nicht zu beobachten. Durch Vergleich der Datenpunkte sind jedoch starke Ähnlichkeiten des Propagationsverhaltens für manche Gruppen von verzweigten Methacrylaten festgestellt worden. In der Vergangenheit ist für Cyclohexyl, Benzyl- und Glycidylmethacrylat Familienverhalten vorgeschlagen worden. $^{\text {[76] }}$ Für Ethylhexyl- Propylheptyl- und Heptadecanylmethacrylat wurde die gleiche Schlussfolgerung getätigt. ${ }^{[74]}$ Somit kann die Hypothese aufgestellt werden, dass Monomere mit ähnlichem sterischen Anspruch ähnliche $k_{\mathrm{p}}$-Werte aufweisen. Wenn tert-Butylmethacrylat als Ausnahme betrachtet wird, könnte auf eine Erniedrigung von $k_{\mathrm{p}}$ mit steigenden sterischen Anspruch geschlossen werden. Dabei reiht sich das im Rahmen dieser Arbeit untersuchte MA-HPBCHC gut in diese Betrachtungsweise ein. Eine naheliegende Erklärung wäre die oben erwähnte Beeinflussung der Rotationsbarriere. Hierbei könnte durch den sterisch anspruchsvollen Rest die Rotationsfreiheit eingeschränkt werden. Dieses entropische Argument müsste sich im präexponentiellen Faktor äußern, welches hier nicht zu beobachten ist.

\subsection{Propagation in nematischer Phase}

Der Einfluss der Verwendung eines nematischen Flüssigkristalls als Lösungsmittel auf die Propagation von MA-HPBCHC wurde durch PLPSEC untersucht. Hierzu wurde das Gemisch BL-087 von der Firma Merck KGaA verwendet. Der Klärpunkt von BL-087 liegt im reinen Gemisch bei $89^{\circ} \mathrm{C}$. Durch Zugabe von MA-HPBCHC (10 wt\%) und Initiator (DMPA, $25 \mathrm{mmol}$ ) wurde der Klärpunkt der flüssigkristallinen Mischung auf $77^{\circ} \mathrm{C}$ herabgesetzt. Aufgrund des deutlich abweichenden Terminierungskoeffizienten von MA-HPBCHC in BL-087 im Vergleich zu 
Toluol (siehe Abschnitt 10) konnten PLP-Strukturen nur in einem begrenzten Pulsfrequenzbereich erhalten werden. Drei erhaltene SECKurven sind in Abbildung 6.5 dargestellt. Hierbei wurde bei $40^{\circ} \mathrm{C}$ und $60{ }^{\circ} \mathrm{C}$ eine $v_{\text {rep }}$ von $1 \mathrm{~Hz}$ und bei $80^{\circ} \mathrm{C}$ eine $v_{\text {rep }}$ von $2 \mathrm{~Hz}$ angewendet.
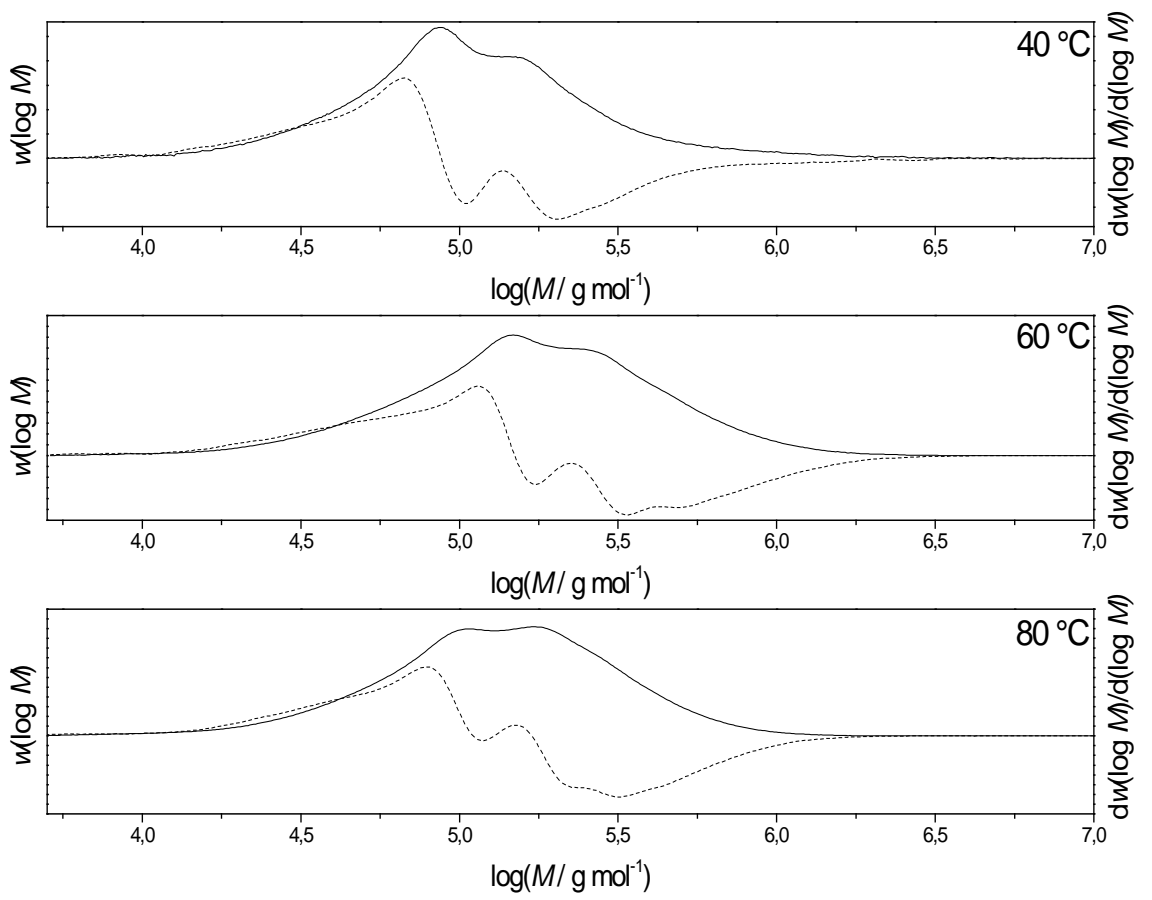

Abbildung 6.5: Molmassenverteilung (durchgezogene Linie) gewonnen aus SEC für Poly(MA-HPBCHC) hergestellt durch PLP (10 wt\% in BL-087, 25 mmol DMPA) und erste Ableitung (gepunktete Linie). Die Kurven bei $40{ }^{\circ} \mathrm{C}$ und $60^{\circ} \mathrm{C}$ wurden mit $v_{\text {rep }}=1 \mathrm{~Hz}$ und die Kurve bei $80^{\circ} \mathrm{C}$ wurde mit $v_{\text {rep }}=2 \mathrm{~Hz}$ aufgenommen.

In den gezeigten PLP-Strukturen weist der Koeffizient $M_{1} / M_{2}$ jeweils einen Wert nahe des Idealwertes 0,5 und erfüllen somit die in Abschnitt 3.6 genannten Richtlinien für Datenkonsistenz. Die Propagationskoeffizienten von MA-HPBCHC in der nematischen Flüssigkeit BL-087 sind mit den zuvor in Toluol erhaltenen Messwerten (siehe Abschnitt 6.3) in Abbildung 6.6 dargestellt. 


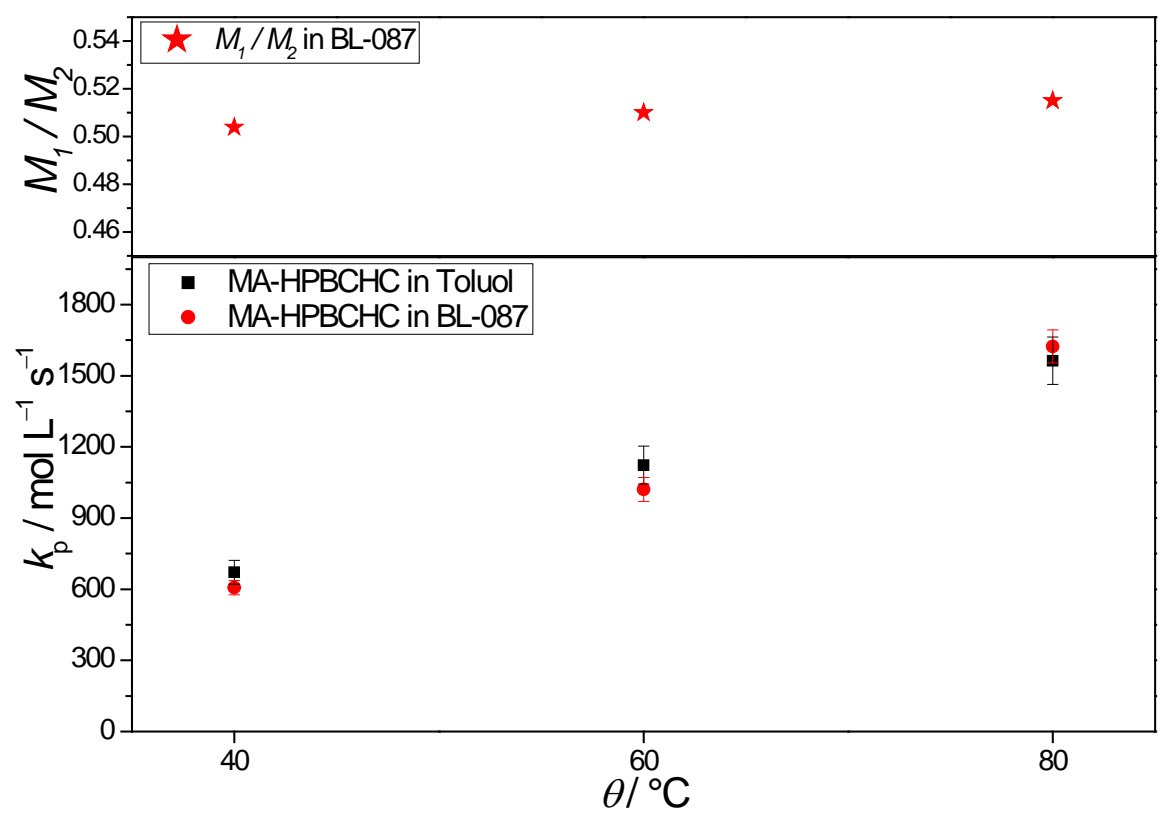

Abbildung 6.6: Propagationskoeffizienten von MA-HPBCHC in BL-087 (Rot) und Toluol (Schwarz) erhalten aus PLP-SEC-Experimenten. Im oberen Bereich sind die entsprechenden Verhältnisse der Molmassen $M_{1} / M_{2}$ für die Messwerte in BL-087 dargestellt.

$\mathrm{Zu}$ beobachten ist keine signifikante Abweichung der Messwerte in Toluol und in BL-087. Dementsprechend kann ein Einfluss der nematischen Phase BL-087 auf den Propagationskoeffizienten von MA-HPBCHC ausgeschlossen werden.

In diesem Kapitel wurde die Bestimmung des Propagationskoeffizienten von MA-HPBCHC in Toluol mittels PLP-SEC vorgestellt. Die mesogene Seitenkette zeigte eine Verringerung von $k_{\mathrm{p}}$ verglichen mit anderen sterisch anspruchsvollen Methacrylaten. Dies verhält sich entgegengesetzt zum beobachtbaren Trend von wachsendem $k_{\mathrm{p}}$ mit wachsender Seitenkette für unverzweigte Alkylsubstituenten. Ein Einfluss der nematischen Phase von BL-087 auf $k_{\mathrm{p}}$ wurde bei PLP-SEC-Experimenten nicht beobachtet. 


\section{Propagation und backbiting von A-HPBCHC}

\subsection{Einleitung}

In diesem Kapitel sollen die Untersuchungen zur Propagation und backbiting von A-HPBCHC vorgestellt werden. Die Reaktionskinetik von Acrylaten stellt, aufgrund der Neigung der SPRs Transferreaktion durchzuführen, eine besondere Herausforderung im Feld der Polymerisationkinetik dar. Der intramolekulare Transfer, wie in Abschnitt 3.5.2 beschrieben, findet als konzertierte [1,5]Wasserstoffübetragunsreaktion statt. Die Radikalfunktionalität wird dabei vom Kettenende auf die dritte Monomereinheit verschoben. ${ }^{[17]}$ Weiterhin wurde mehrfach bei Acrylaten intermolekularer Transfer von Makroradikal auf Polymer beobachtet. ${ }^{[49,80]}$ Die dadurch erzeugten tertiären Radikale (MCRs) können anschließend Homo- und Kreuzterminierung sowie Propagation absolvieren. Im Temperaturbereich über $110^{\circ} \mathrm{C}$ findet zusätzlich die sogenannte $\beta$-Fragmentierung statt, die eine Abspaltung des MCRs in eine gesättigte Doppelbindung und ein SPR erzeugt. Die genannten Reaktionen sind in Abbildung 7.1 und Abbildung 7.2 dargestellt.

Für das entstehende Polymerprodukt bedeuten diese Nebenreaktionen Verzweigungen der Ketten, deren Ausmaß von dem Verhältnis der einzelnen Geschwindigkeitskoeffizienten abhängig ist. Aufgrund der im Vergleich zum SPR niedrigen Propagations- und Terminierungskoeffizienten bedeutet die Bildung von MCRs zusätzlich eine Verlangsamung der Reaktionsrate, sowie insgesamt eine deutliche 
Erhöhung der Komplexität der Reaktionskinetik. Ein vollständiges Verständnis der Kinetik dieser Reaktion kann nur durch Bestimmung der individuellen Geschwindigkeitskoeffizienten der einzelnen Reaktionsschritte erfolgen. Im Folgenden soll die Bestimmung von $k_{\mathrm{p}}^{\mathrm{s}}$ mittels PLP-SEC, von $k_{\mathrm{bb}}$ durch PLP-SEC in Kombination mit rechnergestützten PREDICI ${ }^{\circledR}$ Simulationen und die Anteile an SPRs bzw. MCRs der Radikalpopulation unter quasistationären Bedingungen mittels ESR-Spektroskopie beschrieben werden. Im Rahmen dieser Arbeit wurden alle Experimente mit A-HPBCHC bei Temperaturen bis $70^{\circ} \mathrm{C}$ und Umsätzen bis maximal $5 \%$ durchgeführt. Demensprechend konnte im kinetischen Modell der intermolekulare Transfer und die $\beta$-Fragmentierung vernachlässigt werden.

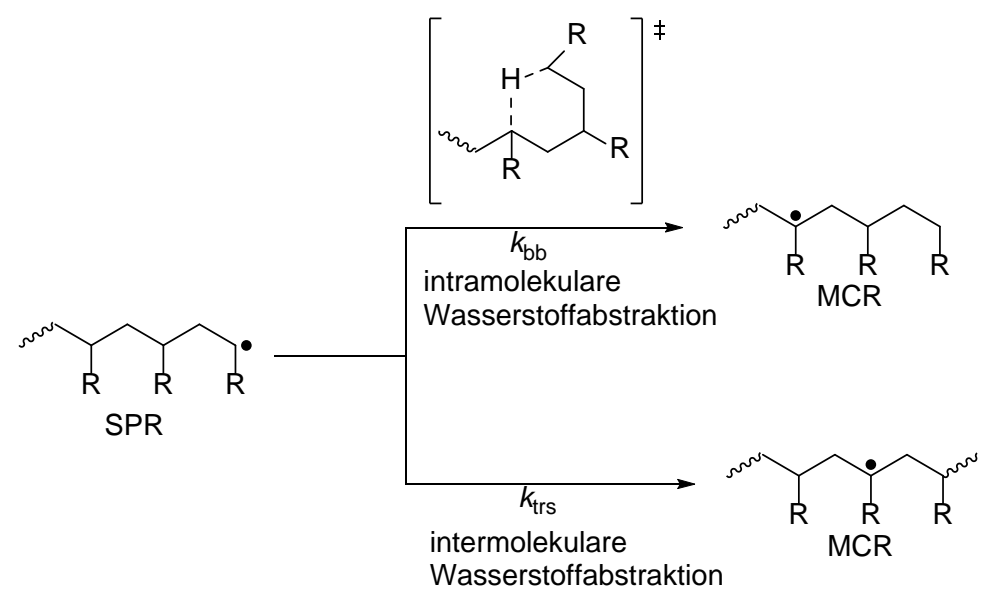

Abbildung 7.1: Reaktionsschema der Transferreaktionen eines SPRs in ein MCR über intra- und intermolekulare Wasserstoffabstraktion für Acrylatpolymerisationen. 


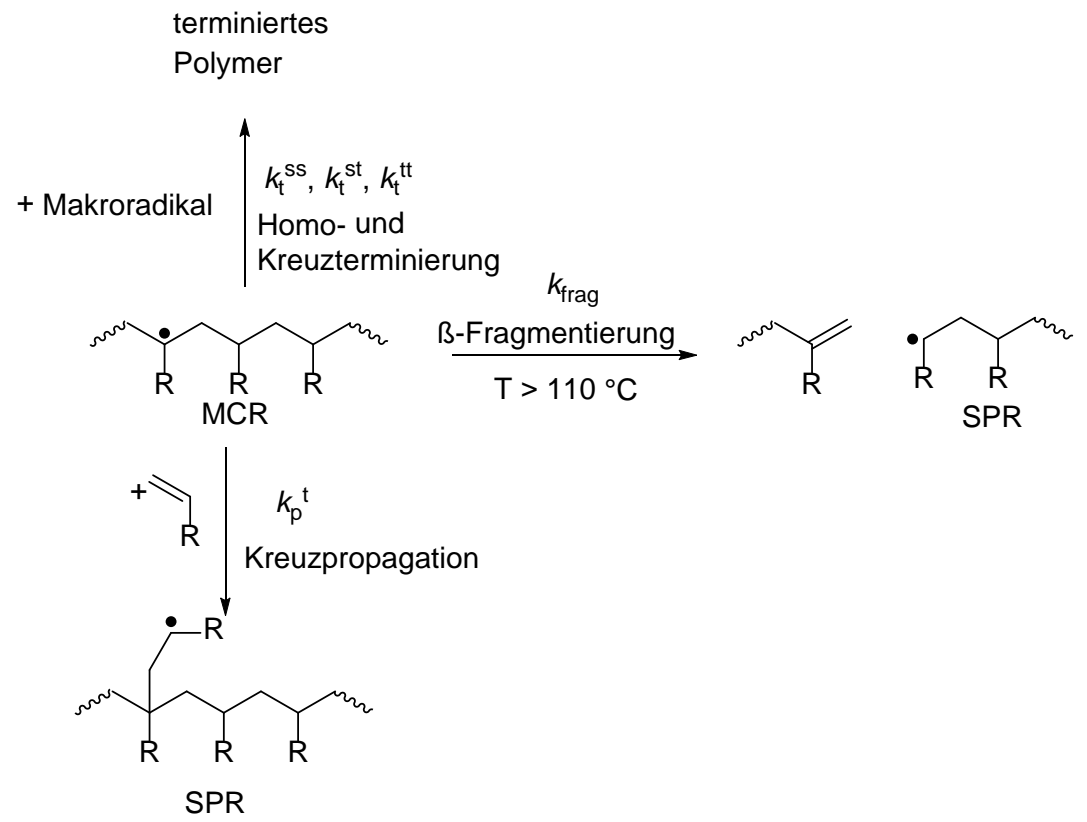

Abbildung 7.2: Darstellung bestimmter Reaktionswege für ein MCR.

\subsection{Propagation der SPRs}

\subsubsection{Strukturanalyse der PLP-SEC Kurven}

Die PLP-SEC Experimente von A-HPBCHC wurden mit 25 wt\% in Toluol durchgeführt. Die Eignung von Toluol für solche Experimente wurde in Abschnitt 6 erläutert. Jede Probe wurde zweimal per SEC analysiert, um ein Maß für die Abweichung durch SEC-Analyse zu ermitteln. Die Pulsanzahl wurde derart eingestellt, dass ein maximaler Umsatz von $5 \%$ erreicht wurde. Somit konnte einerseits genug Polymermaterial für die SECAnalyse erhalten werden und andererseits von einer konstanten Monomerkonzentration ausgegangen werden.

In Abschnitt 7.1 wurden die Auswirkungen von Transferreaktionen auf die Kinetik von Acrylaten beschrieben. Diese Auswirkungen werden bei der Durchführung von pulsfrequenzabhängigen PLP-SEC Experimenten deutlich. In Abbildung 7.3 sind repräsentative Verläufe bei verschiedenen Pulsfrequenzen dargestellt. 

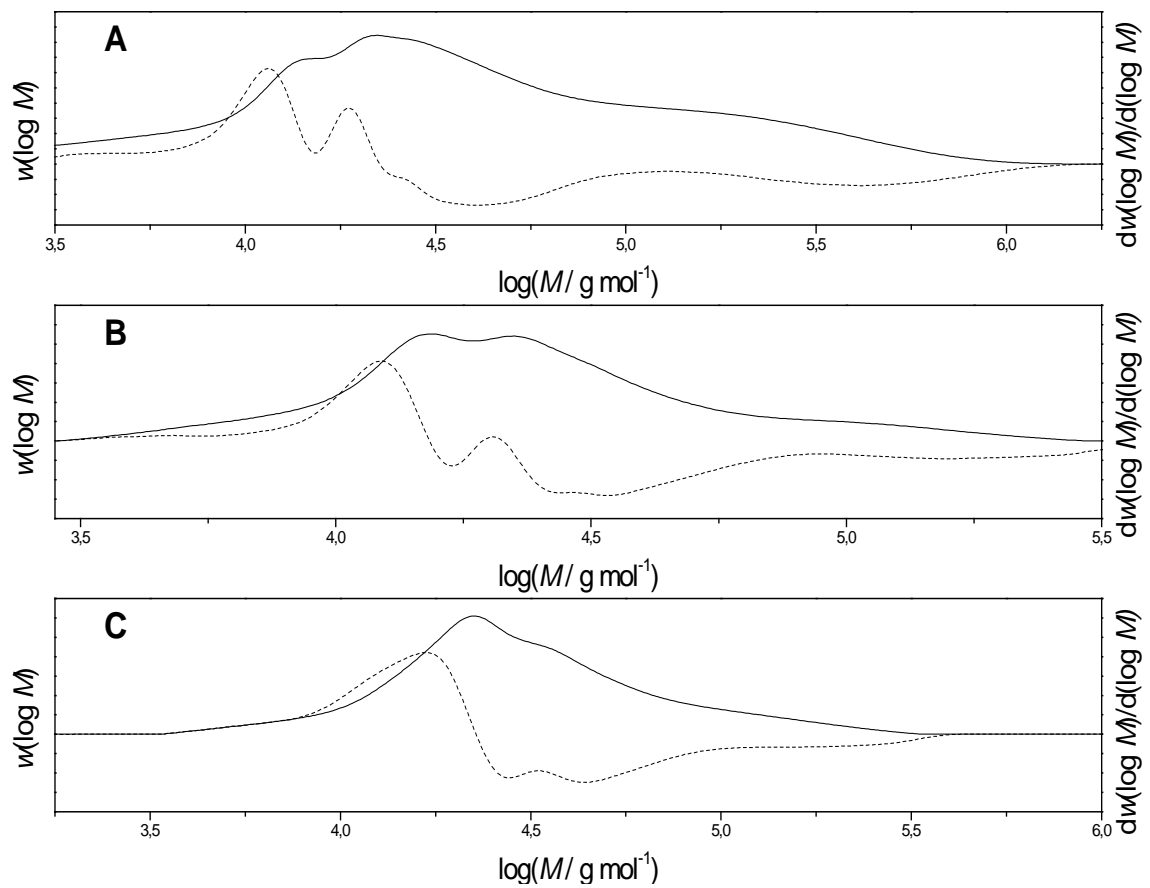

Abbildung 7.3: Molmassenverteilung (durchgezogene Linie) gewonnen aus SEC von Poly(A-HPBCHC), hergestellt durch PLP $\left(30^{\circ} \mathrm{C}, 25 \mathrm{wt} \%\right.$ in Toluol, 50 mmol DMPA) und erste Ableitung (gepunktete Linie). Graph A wurde mit $v_{\text {rep }}=300 \mathrm{~Hz}$, Graph B mit $v_{\text {rep }}=200 \mathrm{~Hz}$ und Graph C mit $v_{\text {rep }}=100 \mathrm{~Hz}$ aufgenommen.

Wie in Abschnitt 3.6 beschrieben, können aus dem POIs der SEC-Kurven Propagationskoeffizienten ermittelt werden. In der Abbildung sind jeweils zwei POIs in jeder Molmassenverteilung zu beobachten. Weiterhin auffällig ist das auf der hochmolekularen Seite der Molmassenverteilung vorliegende Polymer, welches keine PLP Struktur aufweist. Dieses hat aufgrund der deutlich höheren Molekularmasse einen vernachlässigbaren Einfluss auf die POIs der PLP-Struktur. Möglicherweise wurde es durch pre- oder post-PLP Polymerisation oder durch nicht laserpulsterminierte Polymerketten während des PLP Prozesses gebildet.

Die durch diese PLP-SEC Experimente ermittelten Koeffizienten müssen als vermeintliche Propagationskoeffizienten $\left(k_{\mathrm{p}}^{\text {app }}\right)$ betrachtet werden, denn die entstehenden Molmassenverteilung werden vom backbiting beeinflusst. 
In Abbildung 7.4 ist der vermeintliche Propagationskoeffizient $k_{\mathrm{p}}^{\text {app }}$ gegen die angewendete Pulsfrequenz $\left(v_{\text {rep }}\right)$ für eine bei $30^{\circ} \mathrm{C}$ durchgeführte Messreihe aufgetragen.

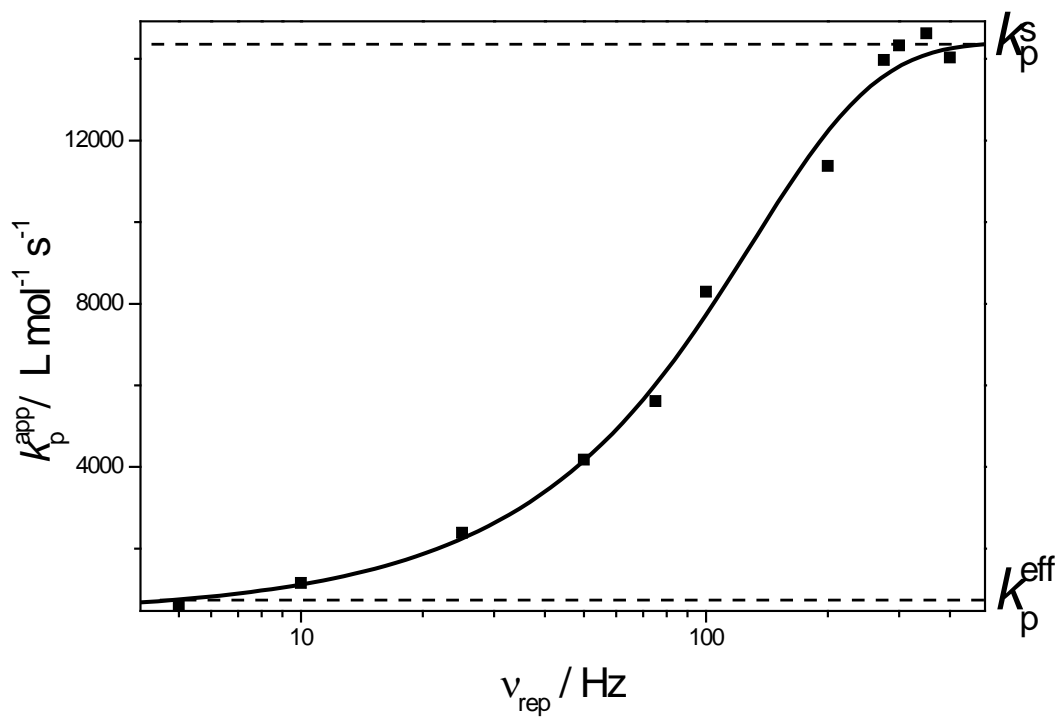

Abbildung 7.4: Auftragung von $\boldsymbol{k}_{\mathrm{p}}^{\mathrm{app}}$ gegen $v_{\text {rep }}$ der PLP-SEC Experimente bei $30^{\circ} \mathrm{C}$ für A-HPBCHC. Die experimentellen Werte wurden zur Veranschaulichung mit einer sigmoidalen Anpassung hinterlegt. Mit gepunkteten Linien sind die Grenzfälle $k_{\mathrm{p}}^{\mathrm{s}}$ und $\boldsymbol{k}_{\mathrm{p}}^{\text {eff }}$ dargestellt.

$\mathrm{Zu}$ beobachten ist der für Acrylate typische sigmoidale Verlauf einer solchen Auftragung, welcher sich durch das Vorkommen von backbiting erklären lässt. Bei sehr hohen $v_{\text {rep }}$ ist die Lebenszeit der Radikale sehr kurz und dementsprechend die Wahrscheinlichkeit für backbiting sehr gering. $\mathrm{Zu}$ sehen ist, dass die Werte in diesem Bereich unabhängig von $v_{\text {rep }}$ sind und um einen Mittelwert schwanken. Wird $v_{\text {rep }}$ verringert, findet vermehrt backbiting statt, die lineare Beziehung zwischen Reaktionszeit und Kettenlänge geht verloren. Je länger die Dunkelzeit desto stärker wird der Wachstumsprozess beeinflusst. In Theorie wird bei sehr niedrigen $v_{\text {rep }}$ erwartet, dass $k_{\mathrm{p}}^{\text {app }}$ ein Plateau erreicht und konstant wird. Daraus könnte der effektive Propagationskoeffizient $k_{\mathrm{p}}^{\text {eff }}$ ermittelt werden, der die Propagationsrate in einem chemisch initiierten Experiment beschreibt. In 
der Praxis ist dies jedoch aufgrund des immer stärker werdenden Einflusses der Terminierung auf die PLP Struktur nicht zu beobachten. Die $v_{\text {rep }}{ }^{-}$ abhängigen Experimente enthalten Informationen bezüglich des Verhältnisses von Kettenwachstum zu Lebensdauer des Radikals. Dies wird in Abschnitt 7.3 zur Bestimmung von $k_{\mathrm{bb}}$ verwendet.

Für die Bestimmung von $k_{\mathrm{p}}^{\mathrm{s}}$, dem Propagationskoeffizient der SPR, wurden die pulsfrequenzunabhängigen Plateaumesswerte herangezogen. Es wurde angenommen, dass der Einfluss von backbiting dort vernachlässigbar ist. Hierbei wurden nur Messwerte mit einer maximalen Abweichung von $5 \%$ verwendet. Die bei $30{ }^{\circ} \mathrm{C}$ aufgenommenen Messwerte sind in Tabelle 7.1 dargestellt. $\mathrm{Zu}$ beobachten ist dass der Quotient $M_{1} / M_{2}$ über dem Idealwert von 0,5 liegt. Dies kennzeichnet den zu erwartenden Einfluss des backbiting auf den zweiten Wendepunkt der SEC-Kurven. Diese Makromoleküle besitzen die doppelte Lebenszeit der beim ersten Wendepunkt terminierten Makromoleküle, dementsprechend kann der Einfluss von backbiting auf sie durch eine Messung mit der halben $v_{\text {rep }}$ abschätzt werden. Wenn aus Tabelle $7.1 k_{\mathrm{p}}^{\text {app }}$ bei $200 \mathrm{~Hz}$ berücksichtigt wird, wird deutlich dass sich der Wert nicht im Plateau der $v_{\text {rep}^{-}}$ unabhängigen Werte befindet. Dementsprechend ist zu erwarten, dass der zweite Wendepunkt bei der $400 \mathrm{~Hz}$ Messung ebenfalls durch backbiting beeinflusst wird. Dementsprechend kann der Idealwert von 0,5 nur erreicht werden, wenn die Messungen mit der doppelten $v_{\text {rep }}$ eines Plateauwerts durchgeführt werden. PLP-Experimente mit $v_{\text {rep }}$ über $400 \mathrm{~Hz}$ führten bei $30{ }^{\circ} \mathrm{C}$ zu Strukturen im LTRL, die keine Auswertung mehr erlauben. Aus diesem Grund wurden die obengenannten Kriterien zur Auswahl der $k_{\mathrm{p}^{\mathrm{s}}}^{\mathrm{s}}$ Werte verwendet. 
Tabelle 7.1: PLP-SEC Ergebnisse der Untersuchungen an A-HPBCHC in Toluol bei $30{ }^{\circ} \mathrm{C}$. $\boldsymbol{k}_{\mathrm{p} 1}$ und $\boldsymbol{k}_{\mathrm{p} 2}$ kennzeichnen die Propagationskoeffizienten die aus dem ersten und zweiten Wendepunkt bestimmt wurden.

\begin{tabular}{ccccc}
\hline $\mathrm{T}$ & $v_{\mathrm{rep}}$ & $k_{p 1}$ & $k_{p 2}$ & $M_{1} / M_{2}$ \\
$/{ }^{\circ} \mathrm{C}$ & $/ \mathrm{Hz}$ & $/ \mathrm{L} \mathrm{mol}^{-1} \mathrm{~s}^{-1}$ & $/ \mathrm{L} \mathrm{mol}^{-1} \mathrm{~s}^{-1}$ & \\
\hline 30,3 & 400 & 14031 & 13066 & 0,54 \\
30,3 & 350 & 14620 & 12619 & 0,58 \\
30,3 & 300 & 14325 & 13016 & 0,55 \\
30,3 & 275 & 13968 & 11223 & 0,62 \\
30,3 & 200 & 11372 & 9434 & 0,60 \\
30,3 & 100 & 8290 & 7501 & 0,55 \\
30,3 & 75 & 5612 & 7398 & 0,38 \\
30,3 & 50 & 4179 & 8168 & 0,26 \\
30,3 & 25 & 2384 & 4859 & 0,25 \\
30,3 & 10 & 1156 & 2586 & 0,22 \\
30,3 & 5 & 630 & 1508 & 0,21 \\
\hline
\end{tabular}

\subsubsection{Temperaturabhängigkeit von $\boldsymbol{k}_{\mathrm{p}}^{\mathrm{s}}$}

Die PLP-SEC Experimente wurden in einem Temperaturbereich zwischen $0{ }^{\circ} \mathrm{C}$ und $70{ }^{\circ} \mathrm{C}$ mit Pulsfrequenzen bis $\mathrm{zu} 900 \mathrm{~Hz}$ durchgeführt. Alle erhaltenen Daten sind in Abschnitt 12 aufgetragen. Aus den temperaturabhängigen Messreihen konnten die Aktivierungsenergie und der präexponentielle Faktor nach Arrhenius bestimmt werden. Die Auftragung ist in Abbildung 7.5 dargestellt. 


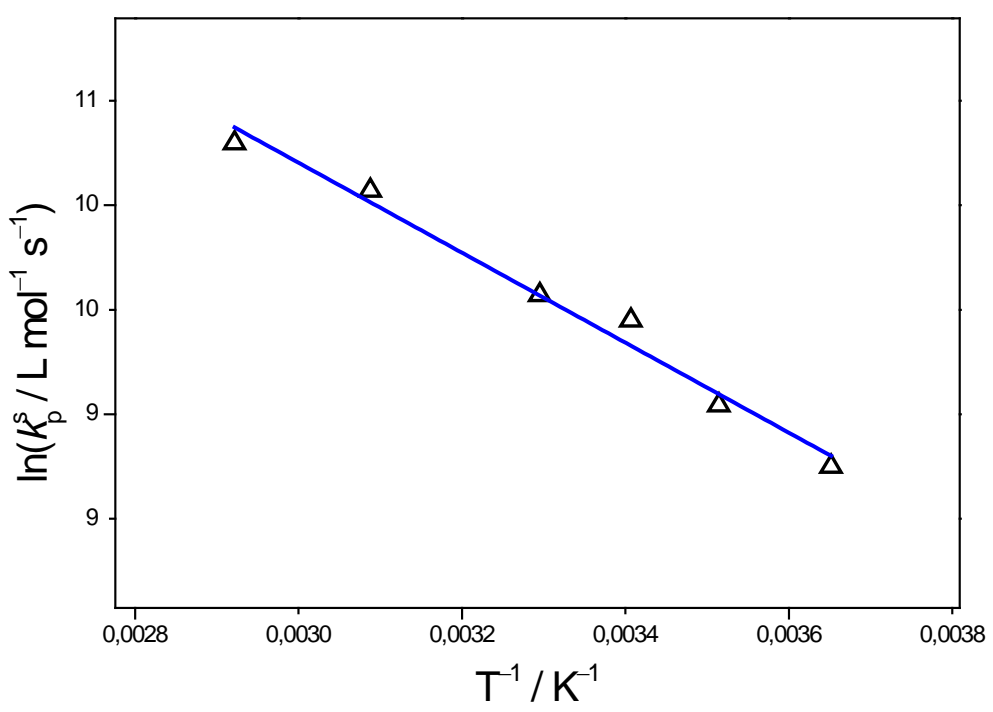

Abbildung 7.5: Arrheniusplot der $\boldsymbol{k}_{\mathrm{p}}^{\mathrm{s}}$-Koeffizienten von A-HPBCHC.

Wie zu erwarten steigt die Propagationsrate mit der Temperatur. Die lineare Anpassung beschreibt den experimentellen Datensatz hinreichend. Die Anpassung liefert folgenden Zusammenhang zur Beschreibung von $k_{\mathrm{p}}^{\mathrm{s}}$ in Abhängigkeit von der Temperatur.

$$
\ln \left(k_{\mathrm{p}}^{\mathrm{s}} / \mathrm{L} \mathrm{mol}^{-1} \mathrm{~s}^{-1}\right)=16,67-2150 /(T / \mathrm{K})
$$

Hierbei kennzeichnet $T$ die absolute Temperatur. Die ermittelte Aktivierungsenergie beträgt somit $17,9 \mathrm{~kJ} \mathrm{~mol}^{-1}$ und der präexponentielle Faktor $1,73 \cdot 10^{7} \mathrm{~L} \mathrm{~mol}^{-1} \mathrm{~s}^{-1}$. Der Fehler der Messungen kann mittels eines $95 \%$ Konfidenzintervalls ermittelt werden. Diese Abschätzung wurde durch die Annahme eines konstanten relativen Fehlers und der Anpassung durch die Methode der kleinsten Quadrate, wie von van Herk vorgeschlagen, durchgeführt. ${ }^{[73]}$ Das Ergebnis dieser Analyse ist in Abbildung 7.6 dargestellt. 


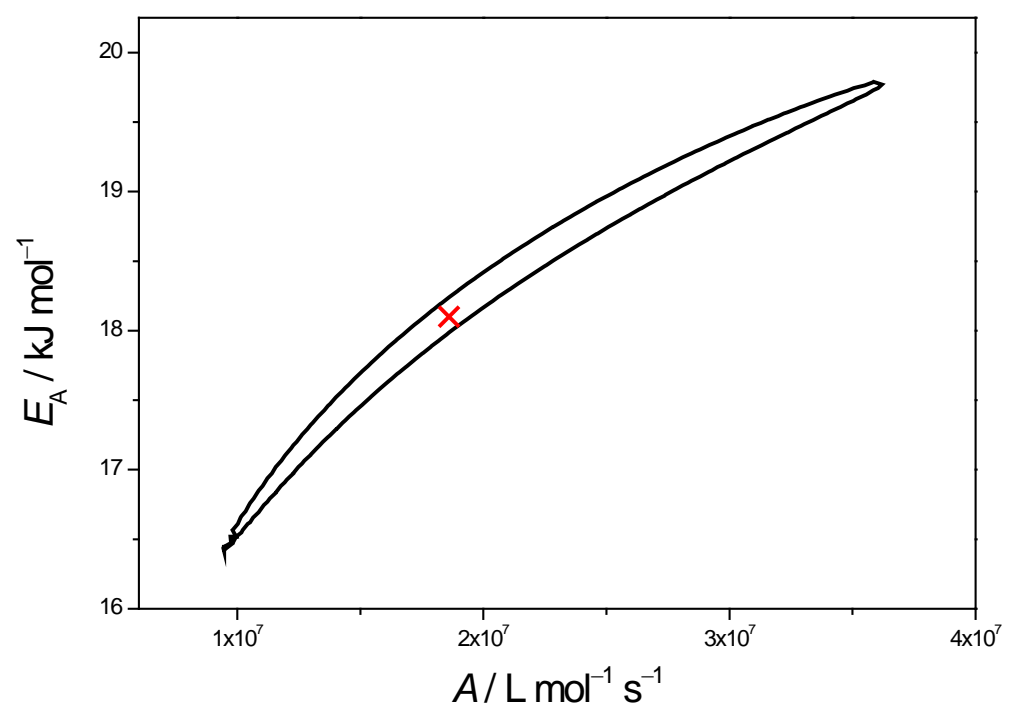

Abbildung 7.6: 95 \% Konfidenzintervall Region der Arrhenius Parameter des Propagationskoeffizienten von A-HPBCHC. Das rote Kreuz gibt das Wertepaar mit der höchsten Wahrscheinlichkeit an.

Es ergibt sich für die Arrhenius Parameter folgende Messungenauigkeit $16,4 \mathrm{~kJ} \mathrm{~mol}^{-1}<E_{\mathrm{A}}<19,8 \mathrm{~kJ} \mathrm{~mol}^{-1}$ und $9,5 \cdot 10^{6} \mathrm{~L} \mathrm{~mol}^{-1} \mathrm{~s}^{-1}<A<3,60 \cdot 10^{7} \mathrm{~L} \mathrm{~mol}^{-1} \mathrm{~s}^{-1}$. Das Fehlerintervall ergibt sich vorwiegend aus der Streuung der Messwerte, während eine Variation des relativen Fehlers sehr ähnliche Verläufe lieferte. Die erhaltenen Fehlerbereiche sind gut mit denen von Datensätze anderer Acrylate vergleichbar. ${ }^{[81]}$

Die Korrelation zwischen Struktur der Seitenkette und Propagationsrate der SPR ist für die Polymerisation von Acrylaten ausführlich in der Literatur beschrieben. ${ }^{[81]}$ Im Allgemeinen kann eine Zunahme von $k_{\mathrm{p}}^{\mathrm{s}}$ für Acrylate mit linearer Seitenkette mit steigender Seitengruppenlänge beobachtet werden. Im Falle von verzweigten Seitenketten konnte bisher noch kein eindeutiger Zusammenhang zwischen $k_{\mathrm{p}}^{\mathrm{s}}$ und der Anzahl an Kohlenstoffatomen in der Seitenkette hergestellt werden. In Tabelle 7.2 sind die Propagationskoeffizienten bei $50{ }^{\circ} \mathrm{C}$ von linearen und verzweigten Acrylaten aufgelistet. Im Falle der verzweigten Acrylaten erfolgt die 
Reihenfolge nach abgeschätzter steigender sterischer Hinderung der Seitenkette.

Tabelle 7.2: Auflistung der Propagationskoeffizienten der SPRs von verschiedenen Acrylaten bei $50{ }^{\circ} \mathrm{C}$. Die Monomere sind unterteilt in lineare und verzweigte Seitenketten tragende Acrylate. Innerhalb dieser Kategorien sind die Substanzen nach abgeschätzter steigender sterischer Hinderung der Seitenkette geordnet.

\begin{tabular}{|c|c|c|c|}
\hline & Seitenkette & $\begin{array}{l}\text { C-Atome in der } \\
\text { Seitenkette }\end{array}$ & $\begin{array}{l}k_{\mathrm{p}}^{\mathrm{s}} \text { bei } 50^{\circ} \mathrm{C} / \\
\mathrm{L} \mathrm{mol}{ }^{-1} \mathrm{~s}^{-1}\end{array}$ \\
\hline \multirow{5}{*}{ Linear } & Methyl $^{[7]}$ & 1 & 22550 \\
\hline & Ethyl $^{[82]}$ & 2 & 26600 \\
\hline & Butyl $^{[8]}$ & 4 & 28240 \\
\hline & Hexyl $^{[82]}$ & 6 & 28570 \\
\hline & Dodecyl $^{[83]}$ & 12 & 31450 \\
\hline \multirow{6}{*}{ verzweigt } & tert-Butyl $^{[56]}$ & 4 & 28270 \\
\hline & Isobornyl $^{[56]}$ & 9 & 20050 \\
\hline & Ethylhexyl $^{[57]}$ & 8 & 25420 \\
\hline & Propylheptyl $^{[74]}$ & 10 & 23370 \\
\hline & Henicosyl ${ }^{[81]}$ & 21 & 25600 \\
\hline & A-НРBCHC & 24 & 22390 \\
\hline
\end{tabular}

Die Beeinflussung des Propagationskoeffizienten durch die Struktur der Seitenkette lässt sich nicht durch eine Veränderung der Elektronendichte an der Radikalfunktionalität erklären. Der auf das radikalische Zentrum wirkende Induktive Effekt ist im Falle der hier aufgelisteten Acrylate aufgrund der räumlichen Trennung durch die Estergruppe sehr ähnlich und daraus folgende Effekte sind vermutlich zu vernachlässigen. Dies wird durch quantenchemische Berechnungen, welche den Einfluss der Kettenlänge auf den Propagationskoeffizienten von Ethylen untersuchten, bestätigt. Hierbei ließ sich eine Änderung des präexponentiellen Faktors mit steigender Kettenlänge vorhersagen. ${ }^{[84]}$ 
Eine Erklärung für das Verhalten linearer Acrylate könnte die Stabilisierung des Makroradikals im Übergangszustand durch die Estergruppe sein. Durch Verlängerung des Alkylrestes wird der Stabilisierungseffekt aufgrund der sinkenden Anzahl an Estergruppen pro Volumeneinheit reduziert. Dies führt zu einer bevorzugten Produktbildung aufgrund der repulsiven Potentialhyperfläche und somit zu einer Erhöhung des Propagationkoeffizienten. ${ }^{[81]}$

Für den Fall verzweigter Acrylate, wie das hier untersuchte A-HPBCHC, sind die Propagationskoeffizienten deutlich niedriger als für dessen lineare Homologe, ausgenommen der Sonderfall des tert-Butylacrylats. Eine Erklärung für die Verringerung von $k_{\mathrm{p}}^{\mathrm{s}}$ könnte die höhere Rotationsbarriere dieser Verbindungen sein. Aus dem sterisch anspruchsvolleren Rest resultiert eine geringere Rotationsfreiheit des Makroradikals, woraus eine Verringerung der Entropie folgt. Das Überwinden der Energiebarriere des Übergangszustandes, welche mittels der Gibbs-Helmholtz-Gleichung beschrieben werden kann, ist somit weniger begünstigt. Dies ist unter der oben ausgeführten Annahme gültig, dass die Seitengruppe einen vernachlässigbaren Einfluss auf die elektronische Umgebung des Übergangzustandes hat und somit auf den enthalpischen Term. Dies resultiert in geringeren Propagationskoeffizienten für sterisch anspruchsvolle verzweigte Acrylate.

Der Grund für die Abwesenheit einer Korrelation zwischen Seitenkettengröße und $k_{\mathrm{p}}^{\mathrm{s}}$ im Falle der verzweigten Acrylaten muss durch weitere empirische Forschung anderer verzweigter Vertreter dieser Substanzklasse ermittelt werden. Die Berücksichtigung sterisch stark anspruchsvoller Seitengruppen ist unumgänglich zum Verständnis der Kinetik der radikalischen Polymerisation dieser Monomerklasse. Die Untersuchung des reaktiven Mesogens A-HPBCHC ist somit ein wichtiger Beitrag zur Vervollständigung des Datensatzes zum Verständnis der Propagation von Acrylaten.

\subsection{Backbiting}

Die Bestimmung des Geschwindigkeitskoeffizienten des backbitings $k_{\mathrm{bb}}$ wurde in der Vergangenheit mittels SP-PLP-ESR und PLP-SEC in Kombination mit PREDICI ${ }^{\circledR}$ Simulationen erreicht. Nikitin et al. führten 
eine ausführliche Untersuchung, in der simulierte Molmassenverteilungen von Butylacrylat mit experimentellen PLP-SEC Strukturen verglichen wurden ${ }^{[85]}$ Hieraus wurde $k_{\mathrm{bb}}$ und der Propagationskoeffizient der MCRs $k_{\mathrm{p}}^{\mathrm{t}}$ ermittelt. Barth et al. verfolgten die charakteristischen ESR-Banden von SPRs und MCRs von Butylacrylat zeitaufgelöst nach Applikation eines Laserpulses. ${ }^{[17]}$ Sie fügten die experimentellen Verläufe in ein PREDICI ${ }^{\circledR}$ Modell ein und passten die Koeffizienten $k_{\mathrm{bb}}, k_{\mathrm{t}}^{\text {st }}, k_{\mathrm{t}}^{\mathrm{ss}}, k_{\mathrm{p}}^{\mathrm{t}}$ an. Beide Untersuchungen lieferten, unter Berücksichtigung des Fehlerintervalls, vergleichbare Ergebnisse in Bezug auf $k_{\mathrm{bb}}$. Wenn und Junkers entwickelten 2016 eine systematische Methode zur Bestimmung von $k_{\mathrm{bb}}$ aus PLP-SEC Datensätze durch Kombination mit PREDICI ${ }^{\circledR}$ Simulationen. ${ }^{[86]}$ Der Vorteil, gegenüber den von Barth durchgeführten Messsungen, ist die deutlich geringere Anzahl an Annahmen. Gegenüber Nikitins Methode erfordert diese Methode deutlich weniger Simulationsaufwand. Im Folgenden soll die Bestimmung von $k_{\mathrm{bb}}$ für A-HPBCHC mit der Methode von Wenn und Junkers vorgestellt werden. Dadurch soll untersucht werden welchen Einfluss eine mesogene, sterisch anspruchsvolle Seitenkette auf $k_{\mathrm{bb}}$ hat.

\subsubsection{Das verwendete Modell}

Das Verfahren beruht auf dem Zusammenhang, der zwischen $k_{\mathrm{bb}}$ und $k_{\mathrm{p}}^{\mathrm{t}}$ besteht: ${ }^{[85]}$

$$
k_{\mathrm{bb}}=[M]\left(\frac{k_{\mathrm{p}}^{\mathrm{s}}}{k_{\mathrm{p}}^{\text {eff }}}-1\right) k_{\mathrm{p}}^{\mathrm{t}}
$$

Dies erlaubt eine Anpassung von $k_{\mathrm{bb}}$ unter Kenntnis von $k_{\mathrm{p}}^{\mathrm{s}}$ und $k_{\mathrm{p}}^{\text {eff }}$ aus den experimentell gewonnenen Messwerten. Um das zu bewerkstelligen wurden die $v_{\text {rep}}$-abhängigen Daten aus den in Abschnitt 7.2 beschriebenen PLP-SEC Experimenten verwendet. Die Verläufe wurden mit einem sigmoidalen Verlauf angepasst wie in Abbildung 7.4 dargestellt. Daraufhin wurde ein Referenzpunkt im sigmoidalen Verlauf bestimmt welcher in der Nähe des Wendepunktes liegen sollte. Dieser Wert fungiert als Referenzpunkt für eine Verschiebung des sigmoidalen Verlaufes entlang der $v_{\text {rep}}$-Achse, welche durch das backbiting zustande kommt. 
Anschließend wurde ein kinetisches Modell mittels PREDICI ${ }^{\circledR}$ erzeugt. Dieses Modell wurde verwendet um die Verschiebung entlang der $v_{\text {rep }}{ }^{-}$ Achse zu simulieren und daraus $k_{\mathrm{bb}} \mathrm{zu}$ bestimmen. Im nachfolgenden wird eine detaillierte Beschreibung des Prozesses gegeben. Das hier verwendete kinetische Modell ist in Tabelle 7.3 dargestellt.

Tabelle 7.3: Kinetisches Schema welches zur Modellierung der Polymerisation von Acrylat verwendet wurde. $I^{*}$ kennzeichnet das initiierende Radikal, SPR $_{i}{ }^{\circ}$ ein sekundäres propagierendes Radikal, $\mathrm{MCR}_{i}{ }^{\circ}$ ein tertiäres propagierendes Radikal, $M$ das Monomer und $D$ das Polymer.

\begin{tabular}{|c|c|c|}
\hline \multicolumn{3}{|c|}{ Kinetisches Schema } \\
\hline Initiierung & $\mathrm{I}^{\circ+\mathrm{M}}$ & $\longrightarrow \mathrm{SPR}_{1}^{\bullet}$ \\
\hline $\begin{array}{l}\text { Propagation der } \\
\text { SPRs }\end{array}$ & $\mathrm{SPR}_{i}^{\bullet}+\mathrm{M}$ & $k_{\mathrm{p}} \longrightarrow \mathrm{SPR}_{i+1}$ \\
\hline Backbiting & $\mathrm{SPR}_{i} \quad k_{\mathrm{bb}}$ & $\mathrm{MCR}_{i}^{\bullet}$ \\
\hline $\begin{array}{l}\text { Propagation der } \\
\text { MCRs }\end{array}$ & $\mathrm{MCR}_{i}+\mathrm{M}$ & $k_{\mathrm{p}}^{\mathrm{t}} \longrightarrow \mathrm{SPR}_{j+1}^{\bullet}$ \\
\hline $\begin{array}{c}\text { Homoterminierung } \\
\text { der SPRs } \\
\text { (Dispoportionierung) }\end{array}$ & $\mathrm{SPR}_{i}^{\bullet}+\mathrm{SPR}_{j}^{\bullet}$ & $\stackrel{k_{\mathrm{t}}^{\mathrm{ss}}}{\longrightarrow} \mathrm{D}_{i}+\mathrm{D}_{j}$ \\
\hline $\begin{array}{c}\text { Homoterminierung } \\
\text { der MCRs } \\
\text { (Dispoportionierung) }\end{array}$ & $\mathrm{MCR}_{i}{ }^{+} \mathrm{MCR}_{j}$ & $k_{\mathrm{t}}^{\mathrm{tt}} \longrightarrow \mathrm{D}_{i}+\mathrm{D}_{j}$ \\
\hline $\begin{array}{l}\text { Kreuzterminierung } \\
\text { (Dispoportionierung) }\end{array}$ & $\mathrm{MCR}_{i}^{\bullet}+\mathrm{SPR}_{j}$ & $k_{\mathrm{t}}^{\mathrm{st}} \longrightarrow \mathrm{D}_{i}+\mathrm{D}_{j}$ \\
\hline
\end{tabular}


Im Modell wurde $k_{\mathrm{bb}}$ durch (7.2) berechnet, was $k_{\mathrm{p}}^{\mathrm{t}}$ zur einzigen unabhängigen Variablen im Modell macht. Dementsprechend kann durch Variation von $k_{\mathrm{p}}^{\mathrm{t}}$ die Verschiebung des sigmoidalen Verlaufs simuliert werden. Zur Simulierung dieses Verlaufs wurde die Entwicklung des Zahlenmittels der Molmasse mit der Reaktionszeit nach einer Einzelpulsinitiierung modelliert. Durch (3.30) lässt sich daraus $k_{\mathrm{p}}^{\text {app }}$ für eine gegebene Wachstumszeit bzw. $v_{\text {rep }}$ eines PLP-Experimentes vorhersagen. Die Verwendung des Zahlenmittels der Molmasse setzt monodisperses Wachstum voraus, welches aufgrund der angewendeten hohen Pulsfrequenzen gültig ist. Weiterhin ist die Verwendung des Zahlenmittels der Molmasse anstatt der POIs aufgrund der Verwendung von Distributionen an Radikalen gerechtfertigt. $k_{\mathrm{p}}^{\mathrm{s}}$ wurde aus den sigmoidalen Verläufen, wie in Abschnitt 7.2 beschrieben, entnommen. Als Schätzung für $k_{\mathrm{p}}^{\text {eff }}$ wurde $k_{\mathrm{p}}^{\text {app }}$ bei $10 \mathrm{~Hz}$ jedes Temperaturverlaufes verwendet. Die Methode zeigte keine Sensitivität bezüglich einer Variation von $k_{\mathrm{p}}^{\text {eff }}$ bei der Bestimmung von $k_{\mathrm{bb}}$, wie schon in der Literatur beobachtet. $^{[86]}$ Die Variation von $k_{\mathrm{p}}^{\text {eff }}$ äußert sich ausschließlich in $k_{\mathrm{p}}^{\mathrm{t}}$. Dementsprechend ist die Ungenauigkeit, welche durch $k_{\mathrm{p}}^{\text {eff }}$ eingeführt wird, zu groß um die erhaltenen $k_{\mathrm{p}}^{\mathrm{t}}$ Werte als verlässlich einzustufen. Für $k_{\mathrm{t}}^{\mathrm{st}}, k_{\mathrm{t}}^{\mathrm{ss}}$ und $k_{\mathrm{t}}^{\mathrm{tt}}$ wurden die von Barth et al. ermittelten Werte für Butylacrylat verwendet. ${ }^{[17]}$ Ein signifikanter Einfluss der Terminierungskoeffizienten auf die errechneten Verläufe konnte nicht beobachtet werden. Bei der Variation der einzelnen Terminierungskoeffizienten um eine Größenordnung wurde eine Abweichung des Ergebnisses von unter $0,5 \%$ beobachtet. In Abbildung 7.7 sind beispielhaft die durch PREDICI ${ }^{\circledR}$ simulierten Verläufe für $30^{\circ} \mathrm{C}$ dargestellt. Hierzu wurde $k_{\mathrm{p}}^{\mathrm{t}}$ variiert um den Einfluss des backbitings auf $k_{\mathrm{p}}^{\mathrm{app}}$ zu ermitteln. 


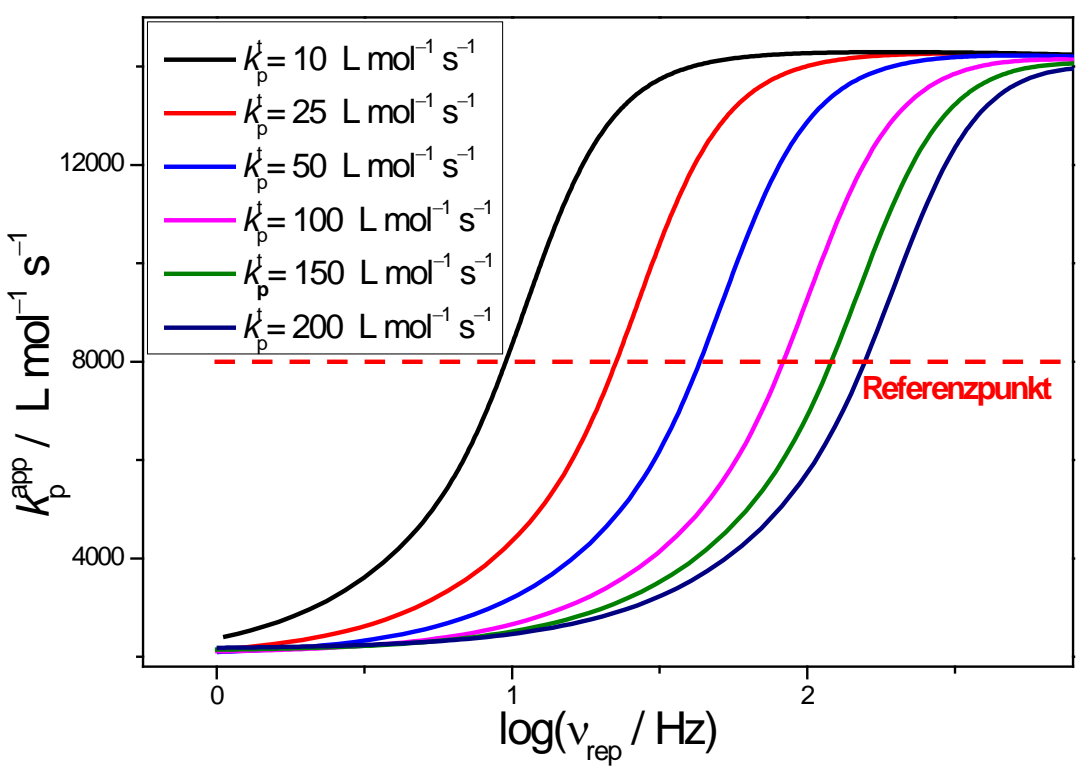

Abbildung 7.7: Mittels PREDICI ${ }^{\circledR}$ simulierte Abhängigkeit des vermeintlichen Propagationskoeffizienten $k_{\mathrm{p}}^{\text {app }}$ von der Pulsfrequenz $v_{\text {rep }}$ bei $30^{\circ} \mathrm{C}$. Hierzu wurde eine Anfangsradikalkonzentration von $1 \cdot 10^{-6} \mathrm{~mol} \mathrm{~L}^{-1}$ angenommen und der Propagationskoeffizient der MCRs $k_{p}^{t}$ von 10 bis $200 \mathrm{Lmol}^{-1} \mathrm{~s}^{-1}$ variiert. Die rot gestrichelte Linie kennzeichnet den Referenzpunkt $\left(k_{\mathrm{p}}^{\mathrm{app}}=8000 \mathrm{~L} \mathrm{~mol}^{-1} \mathrm{~s}^{-1}\right)$ nahe des Wendepunktes der Kurve, welcher zur Bestimmung von $\boldsymbol{k}_{\mathrm{bb}}$ verwendet wurde.

Für die simulierten Verläufe wird der gleiche Referenzpunkt eingeführt wie für die experimentellen Daten. Hierbei wird die jeweilige $v_{\text {rep }}$ bestimmt bei der die Kurve den als Referenzpunkt bestimmten $k_{\mathrm{p}}^{\text {app }}$ Wert schneidet. Anschließend werden die Logarithmen der bestimmten Pulsfrequenz gegen die in der Simulation verwendeten $k_{\mathrm{p}}^{\mathrm{t}}$ Werte aufgetragen. Diese Werte lassen sich gut durch eine exponentielle Funktion anpassen. Anschließend kann die Pulsfrequenz des Referenzpunktes aus dem experimentell bestimmten Verlauf in die exponentiellen Funktion eingesetzt und somit der tatsächliche Wert für $k_{\mathrm{p}}^{\mathrm{t}}$ ermittelt werden. Diese Vorgehensweise ist in Abbildung 7.8 und Abbildung 7.9 dargestellt. Der letzte Schritt ist Bestimmung von $k_{\mathrm{bb}}$ aus $k_{\mathrm{p}}^{\mathrm{t}}$ mittels (7.2). In Theorie ergäbe diese Methode die Parameter $k_{\mathrm{bb}}$ und $k_{\mathrm{p}}^{\mathrm{t}}$. Aus den zuvor beschriebenen Gründen 
können die erhaltenen $k_{\mathrm{p}}^{\mathrm{t}}$ Werte nicht als verlässlich eingestuft werden. Der Vollständigkeit halber soll erwähnt werden, dass die Bestimmung von $k_{\mathrm{p}}^{\mathrm{t}}$ nachträglich durch Messung von $k_{\mathrm{p}}^{\text {eff }}$ erfolgen kann, beispielsweise durch quasistationäre Experimente.

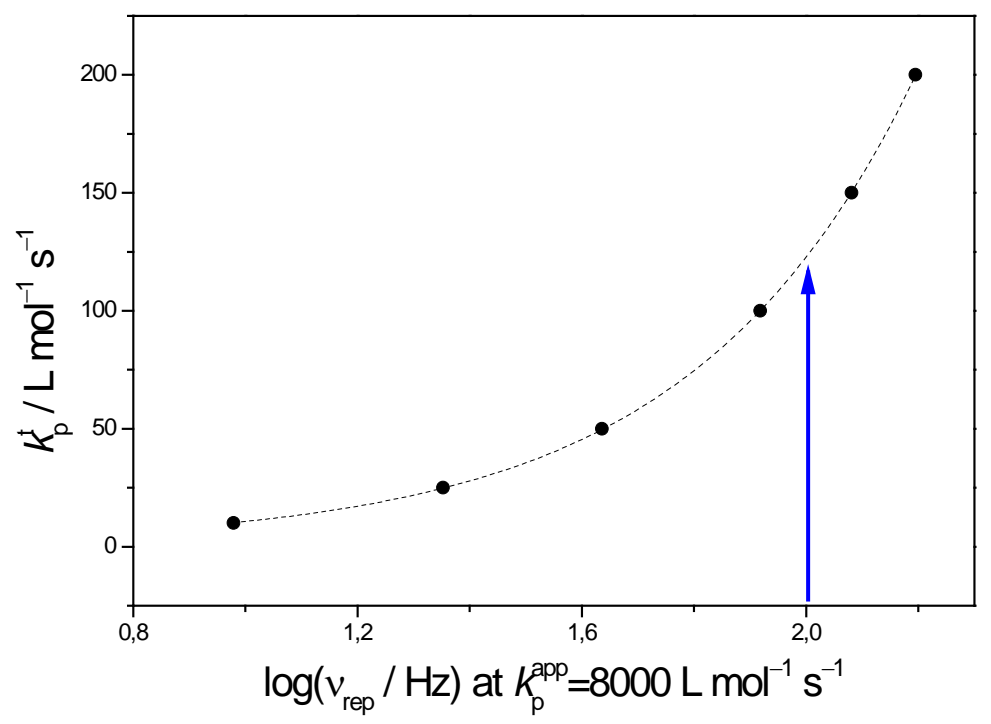

Abbildung 7.8: Logarithmische Auftragung der Pulsfrequenzen, die in den Simulationen beim Referenzpunkt $\left(k_{\mathrm{p}}^{\mathrm{app}}=8000 \mathrm{~L} \mathrm{~mol}^{-1} \mathrm{~s}^{-1}\right)$ ermittelt wurden, gegen den dabei verwendeten Propagationskoeffizienten der MCRs $k_{p}^{t}$ bei $30{ }^{\circ} \mathrm{C}$ (Abbildung 7.7). Die gestrichelte Linie stellt die exponentielle Anpassung dar. Der blaue Pfeil kennzeichnet die in Abbildung 7.9 bestimmte Pulsfrequenz aus den experimentellen Messwerten. 


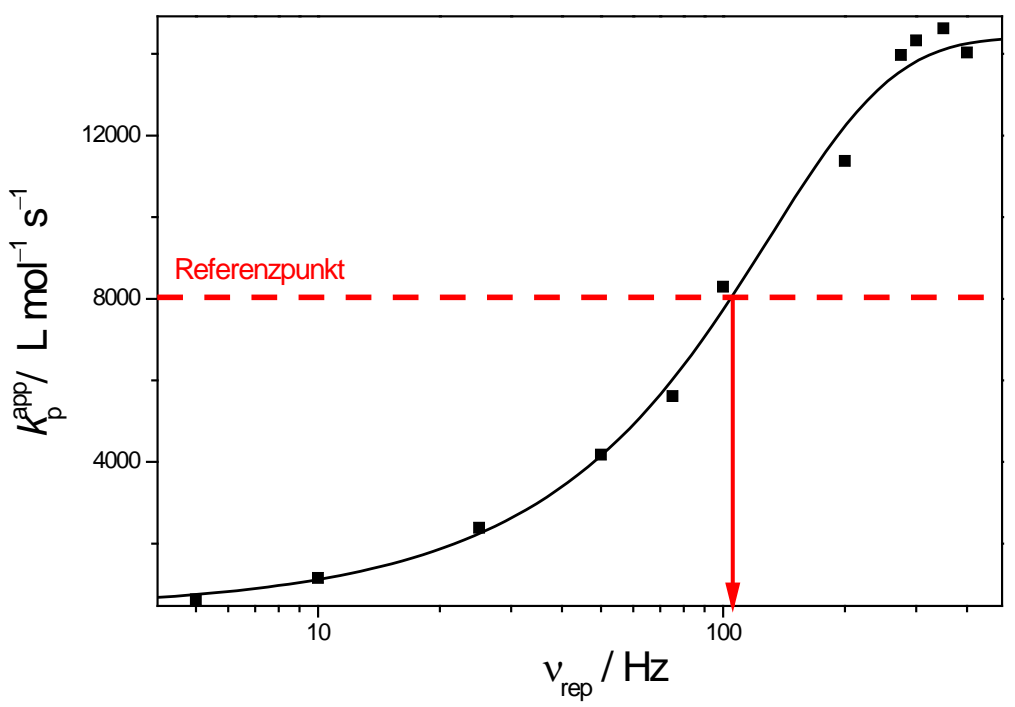

Abbildung 7.9: Auftragung von $\boldsymbol{k}_{\mathrm{p}}^{\mathrm{app}}$ gegen $\boldsymbol{v}_{\text {rep }}$ der PLP-SEC Experimente bei $30^{\circ} \mathrm{C}$. Die experimentellen Werte wurden zur Veranschaulichung mit einer sigmoidalen Anpassung hinterlegt. Die rot gestrichelte Linie kennzeichnet den Referenzpunkt $\left(k_{\mathrm{p}}^{\text {app }}=8000 \mathrm{~L} \mathrm{~mol}^{-1} \mathrm{~s}^{-1}\right)$ nahe des Wendepunktes der Kurve. Der Rote Pfeil markiert die entsprechende Pulsfrequenz die in der exponentiellen Anpassung von Abbildung 7.8 eingefügt wurde.

\subsubsection{Die Temperaturabhängigkeit von $\boldsymbol{k}_{\mathrm{bb}}$}

Die zuvor beschriebene Methode wurde verwendet um $k_{\mathrm{bb}}$ in einem Temperaturbereich von 0 bis $70^{\circ} \mathrm{C}$ zu bestimmen. Die verwendeten experimentellen Daten sind in Abschnitt 12 aufgelistet. Die Temperaturabhängigkeit des Koeffizienten lässt sich durch eine Auftragung nach Arrhenius beschreiben, wie in Abbildung 7.10 dargestellt. 


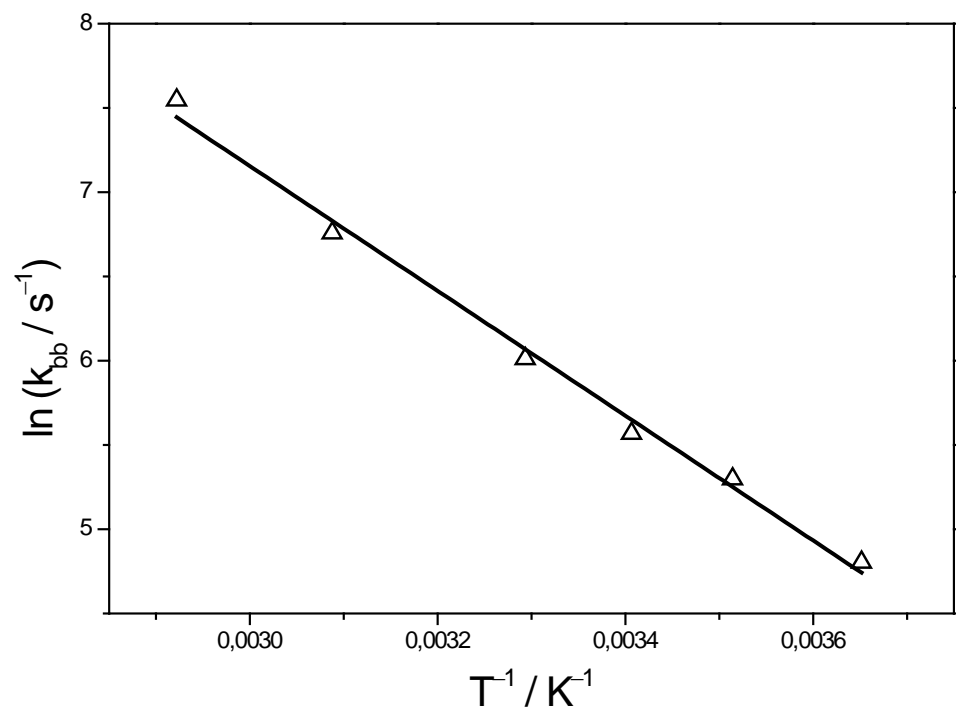

Abbildung 7.10: Arrheniusplot der $\boldsymbol{k}_{\mathrm{bb}}$ Koeffizienten von A-HРBCHC.

Die backbiting-Reaktion zeigt im untersuchten Temperaturbereich eine klare Temperaturabhängigkeit. Die lineare Anpassung nach Arrhenius liefert eine hinreichende Beschreibung des experimentellen Datensatzes. Die Anpassung liefert folgenden Zusammenhang zur Beschreibung von $k_{\mathrm{bb}}$ in Abhängigkeit von der Temperatur.

$$
\ln \left(k_{\mathrm{bb}} / \mathrm{s}^{-1}\right)=18,26-3702 /(T / \mathrm{K})
$$

Hierbei kennzeichnet $T$ die absolute Temperatur. Daraus ergibt sich eine Aktivierungsenergie von $30,8 \pm 1,2 \mathrm{~kJ} \mathrm{~mol}^{-1}$ und ein präexponentieller Faktor von $(8,5 \pm 3) \cdot 10^{7} \mathrm{~s}^{-1}$. In Tabelle 7.4 sind die Ergebnisse dieser Untersuchungen im Vergleich zu den zuvor in der Literatur untersuchten Acrylaten aufgelistet. 
Tabelle 7.4: Auflistung der Literaturwerte für das backbiting verschiedener Acrylate und der zur Bestimmung verwendeten Methode. Weiterhin sind die im Laufe dieser Studie gewonnen Ergebnisse eingetragen. Die Quellen der Ergebnisse sind jeweils in der linken Spalte angegeben.

\begin{tabular}{llllll}
\hline Seitenkette & Methode & $\begin{array}{l}E_{\mathrm{A}} \\
\mathrm{kJ} \mathrm{mol}^{-1}\end{array}$ & $\begin{array}{l}A \cdot 10^{-7} \\
\mathrm{~s}^{-1}\end{array}$ & $\begin{array}{l}k_{\mathrm{bb}}\left(50{ }^{\circ} \mathrm{C}\right) \\
/ \mathrm{s}^{-1}\end{array}$ \\
\hline Butyl $^{[17]}$ & ESR & $34,7 \pm 0,8$ & $16 \pm 7$ & 390 \\
Butyl $^{[85]}$ & PLP-SEC & $31,7 \pm 2,5$ & $4,84 \pm 0,29$ & 370 \\
tert-Butyl $^{[86]}$ & PLP-SEC & $25,9 \pm 2,2$ & $0,4 \pm 0,2$ & 270 \\
A-HPBCHC & PLP-SEC & $30,8 \pm 1,2$ & $8,5 \pm 3$ & 900 \\
\hline
\end{tabular}

Es lässt sich beobachten, dass das hier untersuchte A-HPBCHC im Vergleich zu zuvor untersuchten Acrylaten höhere backbiting-Raten aufweist. Bei $50{ }^{\circ} \mathrm{C}$ ist $k_{\mathrm{bb}}$ ca. um den Faktor 2,4 höher als für Butylacrylat und ca. um den Faktor 3,3 höher als für tert-Butylacrylat. Diese Tendenz wird durch ESR-spektroskopische Messungen unterstützt, die in Abschnitt 7.4 beschrieben sind. Der zur Verfügung stehende Datensatz ist ungenügend um klare Aussagen bezüglich des Ursprungs der erhöhten Rate zu tätigen. Ob die sterisch anspruchsvolle Seitenkette einen Einfluss auf die Aktivierungsenergie oder des präexponentiellen Faktors hat, ist nicht zu erschließen. Eine mögliche Erklärung für die erhöhte Neigung von A-HPBCHC zu backbiting könnte eine erhöhte Stabilität des entstehenden MCRs aufgrund der sterisch anspruchsvollen Seitenketten sein.

Die hier vorgestellten Untersuchungen zeigen die Notwendigkeit jedes einzelne Acrylat in Bezug auf backbiting zu untersuchen. Die Struktur der Seitenkette beeinflusst $k_{\mathrm{bb}}$ augenscheinlich im signifikanten Maße.

\subsection{Zusammensetzung der Radikalpopulation}

Elektronenspinresonanz bietet direkten Zugang zur Struktur von Radikalen und wurde deshalb zur Untersuchung der beteiligten Radikalspezies in der Polymerisation von A-HPBCHC verwendet. Alle Spektren in diesem 
Abschnitt wurden bis zu maximalen Umsätzen von $5 \%$ gemessen. Innerhalb dieses Bereiches konnte keine Umsatzabhängigkeit der Spektren beobachtet werden.

In der Vergangenheit ist eine starke Temperaturabhängigkeit der ESRSpektren von Acrylaten beobachtet worden. ${ }^{[87,88]}$ Dies kann durch die Überlappung mehrerer ESR-aktiven Spezies innerhalb des Systems erklärt werden.

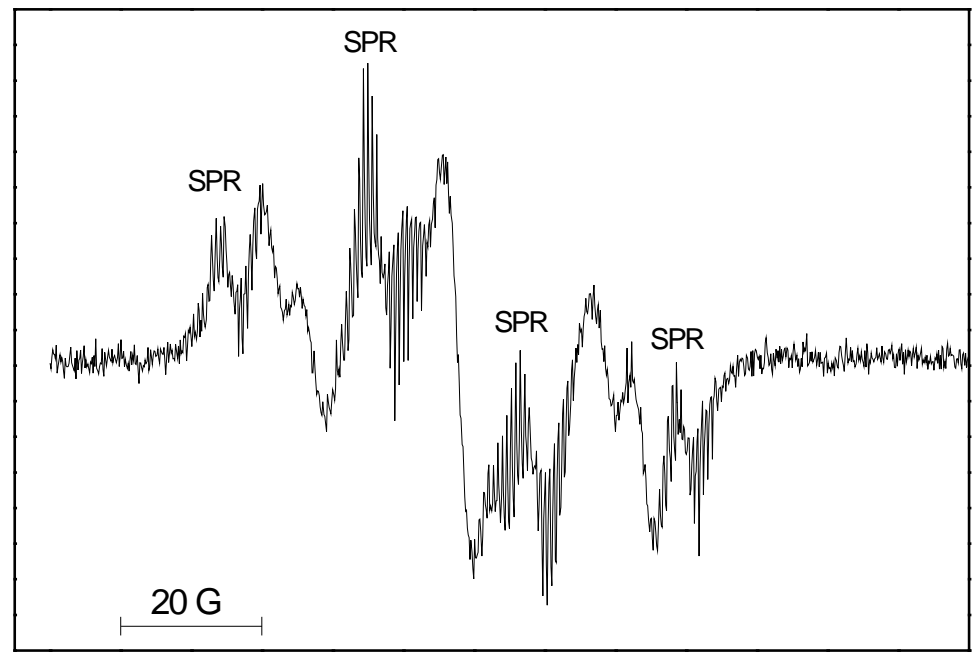

Abbildung 7.11: ESR-Spektrum von A-HPBCHC in Toluol (25 wt\%) bei $-10{ }^{\circ} \mathrm{C}$. Die Radikale wurden mittels Laserpulsbestrahlung des Initiators Darocure $^{\circledR} 1173\left(1,5 \cdot 10^{-2} \mathrm{~mol} \mathrm{~L}^{-1}\right)$ mit einer Pulsfrequenz von $20 \mathrm{~Hz}$ erzeugt. Mit SPR sind die Banden gekennzeichnet die den kurzlebigen SPRs zugeteilt wurden.

Die Stabilität der beteiligten Spezies unterscheidet sich stark, wie aus dem in Abbildung 7.11 dargestelltem ESR-Spektrum von A-HPBCHC bei $-10{ }^{\circ} \mathrm{C}$ beobachtet werden kann, welches mittels periodischer Laserpulse (20 Hz) initiiert wurde. Darin können innerhalb des Spektrums Bereiche unterschieden werden, die in Bezug auf die y-Achsen-Varianz voneinander abweichen. Die Bereiche mit periodischen hohen $\mathrm{Zu}$ - und Abnahmen an Intensität, welche deutlich höher als das eigentliche Rauschen des Spektrums sind, wurden in der Abbildung mit SPR markiert. Diese periodischen Strukturen kommen durch die sehr schnelle Terminierung einer Spezies nach Eintreffen des Laserpulses zustande. Die Bereiche, die 
solchen periodischen Schwankungen entbehren, entsprechen ESR-aktiven Spezies die deutlich langsamere Terminierung aufweisen, welche bei der angewendeten Pulsfrequenz und Messdauer keine Abweichungen in der Spektrenintensität erzeugt. Dieses Verhalten kann durch das Vorkommen von SPRs und MCRs im Reaktionssystem erklärt werden. MCRs sind tertiäre Radikale die deutlich niedrigere Terminierungsraten erwarten lassen als SPRs. Dementsprechend können die Banden mit hoher Periodizität den SPRs und der Rest den MCRs zugeordnet werden.

Aus den Untersuchungen in Abschnitt 7.2 und 7.3 wird ersichtlich, dass die Aktivierungsenergie des backbitings deutlich höher als die des Propagationsschrittes ist. Dementsprechend ist bei niedrigen Temperaturen ein höherer Anteil an SPRs zu erwarten. In Abbildung 7.12 ist das ESRSpektrum von A-HPBCHC in Toluol (25 wt\%) bei $-50{ }^{\circ} \mathrm{C}$ dargestellt.

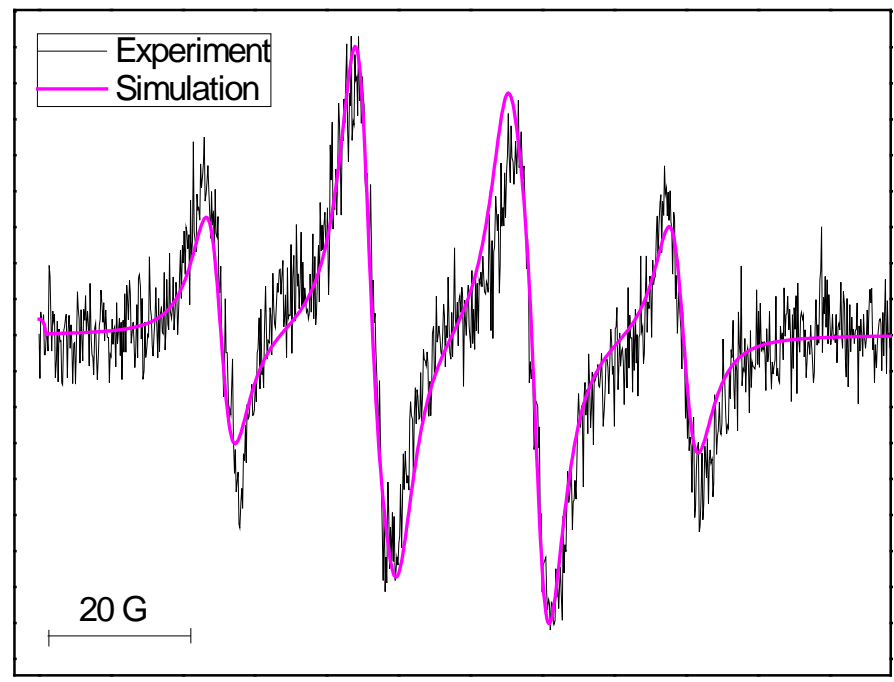

Abbildung 7.12: Schwarz: Experimentelles ESR-Spektrum von A-HPBCHC in Toluol (25 wt \%) bei $-50{ }^{\circ} \mathrm{C}$. Die Radikale wurden mittels kontinuierlicher Bestrahlung des Initiators Darocure ${ }^{\circledR} 1173\left(1,5 \cdot 10^{-2} \mathrm{~mol} \mathrm{~L}^{-1}\right)$ durch eine Quecksilberbogenlampe erzeugt. Violett: Simuliertes Spektrum mit Hyperfeinkopplungskonstanten 2,36 mT und 2,08 mT.

Das Spektrum zeigt die gleichen vier Banden welche in der Vergangenheit als charakteristisch für SPRs in Acrylatpolymerisationen interpretiert wurden. ${ }^{\text {[87-89] }}$ Zur Simulation des Spektrums wurde eine Kopplung des Radikals mit einem $\alpha$-Wasserstoffatom und zwei $\beta$-Wasserstoffatomen mit 
den Hyperfeinkopplungskonstanten 2,36 mT und 2,08 mT angenommen. Diese Werte stimmen mit den für andere Acrylaten berichteten Werten überein. ${ }^{[87-89]}$ Weiterhin ist auffällig, dass diese vier Banden den in Abbildung 7.11 zugeordneten SPR Banden in Bezug auf die Magnetfeldstärke entsprechen. Das gleiche Spektrum zeigen in den Untersuchungen von Kajiwara und Kamachi sekundäre Dimere Modelradikale von tert-Butylacrylat die kein backbiting absolvieren können. ${ }^{[90]}$ Die auftretenden vier Banden können somit als Ergebnis der Überlappung eines Dubletts von Tripletts durch Bandenverbreiterung erklärt und dementsprechend den SPRs zugeordnet werden.
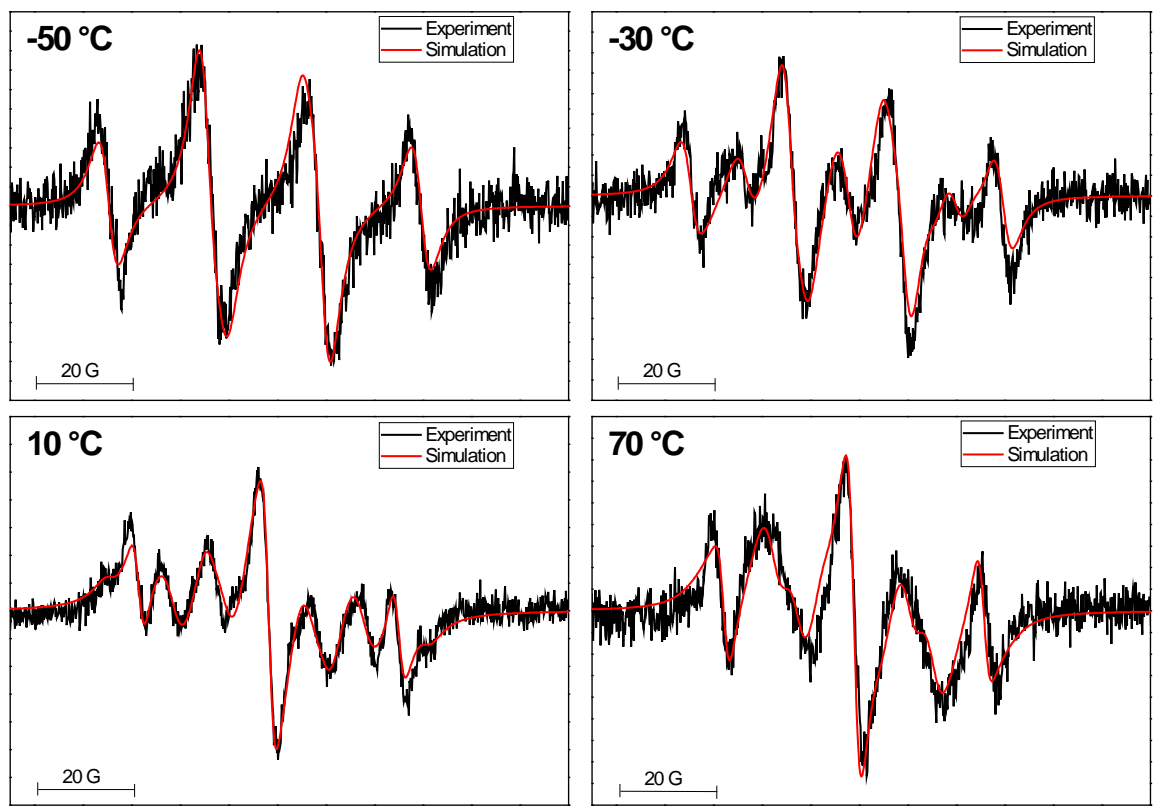

Abbildung 7.13: Schwarz: Experimentelle ESR-Spektren von A-HPBCHC in Toluol (25 wt \%) bei $-50^{\circ} \mathrm{C},-30^{\circ} \mathrm{C}, 10^{\circ} \mathrm{C}$ und $70^{\circ} \mathrm{C}$. Die Radikale wurden mittels kontinuierlicher Bestrahlung des Initiators Darocure ${ }^{\circledR} 1173$ $\left(1,5 \cdot 10^{-2} \mathrm{~mol} \mathrm{~L}^{-1}\right)$ durch eine Quecksilberbogenlampe erzeugt. Rot: Simulierte Spektren.

Wird die Temperatur erhöht, äußert sich der Einfluss der MCRs auf die ESR-Spektren immer dominanter. In Abbildung 7.13 sind Spektren von $-50{ }^{\circ} \mathrm{C}$ bis $70^{\circ} \mathrm{C}$ aufgetragen. $\mathrm{Zu}$ beobachten ist die Zunahme eines zentralen Peaks welcher charakteristisch für MCRs ist. Dieser erlaubt die 
Bestimmung von MCR-Anteilen auch bei niedrigen Konzentrationen. Charakteristisch für Acrylat-Spektren bei hoher Temperatur ist das Auftreten von sieben Banden (siehe Abbildung 7.13). ${ }^{[91-94]}$ Diese lassen sich als Triplett von Tripletts interpretieren die durch Bandenverbreiterung in ein sieben Banden Spektrum münden. Diese Annahme erscheint aufgrund einer, durch gehinderte Dynamik der Ketten unterschiedliche molekularer Umgebung für die jeweiligen $\beta$-Wasserstoffatompaare und somit abweichende Kopplungskonstanten, sinnvoll. Die beim backbiting von Acrylaten bei weitem dominanter vorkommenden kurzkettigen Verzweigungen bedeuten eine Kurzketten- und eine Langkettenverzweigung auf jeder Seite des MCR-Radikals und somit eine abweichende chemische Umgebung. Wie zuvor in der Literatur beobachtet lässt sich das experimentelle Spektrum mit höherer Übereinstimmung simulieren wenn zusätzlich ein drei Banden MCR-Spektrum addiert wird. ${ }^{\text {[91-94] }}$ Diese Spezies kann als MCR mit einer vorwiegenden Kopplung an zwei Wasserstoffatome interpretiert werden, während die Kopplung zu den zwei weiteren so gering ist, dass sie in der hier verwendeten Apparatur nicht aufgelöst werden kann. Die geringe Kopplung käme zustande durch eine Anordnung der Wasserstoffatome nahe der Knotenebene des einfach besetzten Orbitals aufgrund einer gehinderten Rotation um die $C_{\alpha}-C_{\beta}$ Bindung. Nach der Gleichung von Heller-McConnel (7.4) ist die Kopplungskonstante $a(\beta-\mathrm{H})$ abhängig vom Winkel $\Theta$ zwischen einfach besetzten p-Orbital und dem $\alpha$-Orbital der $\mathrm{C}_{\beta}$-H Bindung. $A(\mathrm{H})$ ist hier eine Proportionalitätskonstante.

$$
a(\beta-\mathrm{H})=A(\mathrm{H}) \cdot \cos ^{2}(\Theta)
$$

Zur Bestimmung der Kopplungskonstanten der drei und sieben Banden MCR-Spektren wurde das bei der höchsten Temperatur $\left(70^{\circ} \mathrm{C}\right)$ experimentell aufgenommene Spektrum analysiert. In diesem Fall ist der Einfluss an SPRs am geringsten. Das Spektrum wurde mittels MATLAB ${ }^{\circledR}$ in Kombination mit EASYSPIN ${ }^{\circledR}$ unter Verwendung des LevenbergMarquardt-Algorithmus angepasst. Die entnommenen Kopplungskonstanten 1,65 mT und 1,15 mT für die sieben Banden Spezies und 2,8 mT für die drei Banden Spezies liegen sehr nah an den für 
Butylacrylat und Dodecylacrylat bestimmten Werten. Das Fitten intermediärer Temperaturen zeigte eine Temperaturabhängigkeit der Kopplungskonstanten der sieben Banden Spezies, wobei die Summe der Kopplungskonstante immer bei 2,8 mT lag (siehe Tabelle 7.5).

Tabelle 7.5: Kopplungskonstanten für A-HPBCHC, bestimmt aus den experimentellen ESR-Spektren.

\begin{tabular}{cccccc}
\hline Radikal & $\theta /{ }^{\circ} \mathrm{C}$ & $\mathrm{a}(\alpha-\mathrm{H})$ & $\mathrm{a}(\beta-\mathrm{H})$ & $\mathrm{a}\left(\beta_{1}-\mathrm{H}\right)$ & $\mathrm{a}\left(\beta_{2}-\mathrm{H}\right)$ \\
\hline SPR & -50 & 2,36 & 2,08 & - & - \\
\hline $\begin{array}{c}\text { MCR } \\
\text { 3-Banden }\end{array}$ & -50 bis 70 & - & 2.8 & - & - \\
\hline MCR & -30 & & 2 & 0.8 \\
7-Banden & 10 & & 1.95 & 0.85 \\
& 70 & & 1.65 & 1.15 \\
\hline
\end{tabular}

Dieses Verhalten wurde zuvor für Butylacrylat ebenfalls beobachtet. ${ }^{[87]}$ Mit steigender Temperatur nähern sich die Kopplungskonstanten beider $\beta$ Wasserstoffpaare immer weiter an, was vermutlich einer sinkenden Rotationshinderung mit steigender Temperatur zuzuschreiben ist. Bei der Simulation der experimentellen Spektren muss zwangsläufig eine Gewichtung der einzelnen Spezies vorgenommen werden, welche in diesem Fall durch Fitten mittels des Levenberg-Marquardt-Algorithmus durchgeführt wurde. Auf diese Art und Weise lässt sich der Anteil an MCRs in Bezug auf die gesamte Radikalpopulation bei für quasistationäre Bedingungen bestimmen. Die Ergebnisse dieser Untersuchungen für die Polymerisation von A-HPBCHC in Toluol (25 wt\%) sind in Abbildung 7.14 dargestellt. Zusätzlich sind die Literaturwerte von Butylacrylat zum Vergleich abgebildet. 


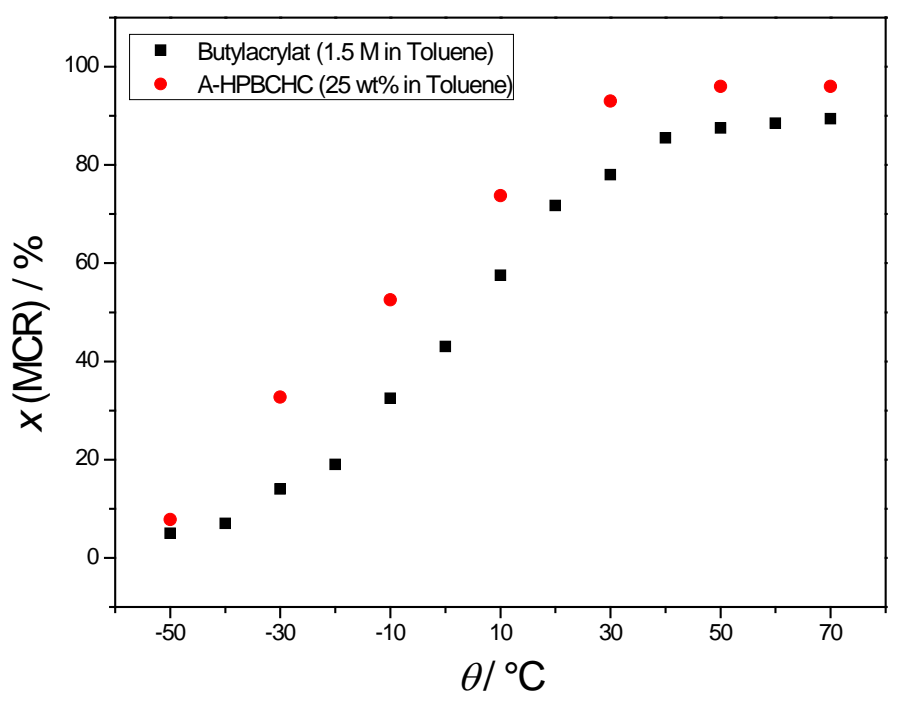

Abbildung 7.14: Prozentueller Anteil an MCRs bei der Polymerisation von
A-HPBCHC in Toluol (25 wt\%) in Abhängigkeit von der Temperatur.
Ermittelt durch Komponentenanalyse der experimentellen ESR-Spektren. Die
Werte für Butylacrylat sind der Literatur entnommen. ${ }^{[8]}$

Die Ergebnisse zeigen deutlich, dass die Polymerisation von A-HPBCHC in hohem Maße von der Bildung von MCRs beeinflusst wird. Der Temperaturbereich, der für einen Großteil der Anwendungen von Bedeutung ist, zeigt MCR-Anteile von über $90 \%$. Der Verlauf ähnelt dem sigmoidalen Verlauf der in der Vergangenheit für Butylacrylat und Acrylamid gefunden wurde ${ }^{[87,88]}$ Die Anteile an MCRs sind jedoch für jede Temperatur signifikant höher, ebenso wie die Plateauwerte. Die Analyse der Gründe ist nicht trivial. Nikitin und Hutchinson führten den Term (7.5) zur Beschreibung der MCR-Anteile unter quasistationären Bedingungen ein. ${ }^{[53]}$ Dieser Term nimmt eine schnellere MCR-Kreuzpropagation als MCR Terminierung und die Langketten-Näherung an welche Terminierung von MCRs negiert.

$$
x_{\mathrm{MCR}}=\frac{k_{\mathrm{bb}}}{k_{\mathrm{bb}}+k_{\mathrm{p}}^{\mathrm{t}} \cdot c_{\mathrm{M}}}
$$


Die Literaturwerte für Butylacrylat lassen sich mit diesem Zusammenhang nicht ausreichend beschreiben. Kattner und Buback schlugen eine Erweiterung dieser Gleichung vor, welche die Kreuzterminierung von MCRs und SPRs berücksichtigt: ${ }^{\text {[88] }}$

$$
x_{\mathrm{MCR}}=\frac{k_{\mathrm{bb}}}{k_{\mathrm{bb}}+k_{\mathrm{p}}^{\mathrm{t}} \cdot c_{\mathrm{M}}+k_{\mathrm{t}}^{\mathrm{st}} \cdot c_{\mathrm{SPR}}} .
$$

Dieser Zusammenhang beschreibt die Literaturdaten für Butylacrylat mit deutlich höherer Genauigkeit. Es erfordert jedoch einen kompletteren Datensatz mit Informationen über die Kreuzterminierung im System und kann somit nicht zum Verständnis der A-HPBCHC Spektren verwendet werden. Unter der Annahme dass die Kreuzterminierung in diesem System ähnlich wie bei Butylacrylat verhält und dass $k_{\mathrm{p}}^{\mathrm{t}}$ ein Familienverhalten zeigt, bestätigen die hier vorgeführten Ergebnisse eine erhöhte backbiting Rate für A-HPBCHC im Vergleich zu Butylacrylat.

In diesem Kapitel wurden die durchgeführten Untersuchungen zur Kinetik von A-HPBCHC vorgestellt. Es wird deutlich, dass A-HPBCHC stärker von backbiting betroffen ist als zuvor untersuchte Acrylate. Diese Tatsache in Kombination mit der vergleichsweise geringen Propagationsgeschwindigkeit der SPRs bedeutet eine hohe Anzahl an Verzweigungen im Produkt der Homopolymerisation dieses Monomers. 


\section{Propagation von FA-HPBCHC}

\subsection{Einleitung}

Polyfluoroacrylate sind aufgrund ihrer hohen Hitzebeständigkeit und Schlagfestigkeit von wissenschaftlichem Interesse. ${ }^{[95]}$ Aufgrund der komplexen und kostenintensiven Syntheserouten sind industrielle Anwendungen bis heute selten. ${ }^{[95]}$ Daraus erklärt sich, dass bis zum jetzigen Zeitpunkt nur wenige kinetischen Untersuchungen für diese Monomergruppe durchgeführt wurden. Eine Studie von Yamada et al. ist dabei eine der wenigen Ausnahmen. ${ }^{[96]}$ Sie führten Untersuchungen zu Ethylfluoroacrylat (EFA) durch und bestimmten den Propagationskoeffizienten und Terminierungskoeffizienten durch die Rotating-Sector-Methode bei $30{ }^{\circ} \mathrm{C} .{ }^{[96]}$

Im Rahmen dieses Kapitels wird die Bestimmung des Propagationskoeffizienten des mesogenen Fluoroacrylats FA-HPBCHC mittels PLP-SEC beschrieben. Aufgrund des hohen Schmelzpunktes von FA-HPBCHC wurden die Untersuchungen in Toluol (20 wt\%) durchgeführt. Die Eignung von Toluol als Lösungsmittel ist in Abschnitt 5.1 erklärt. Die Analyse der Polymer-Produkte erfolgte durch SEC mit Brechungsindexdetektion. Die Molmassenverteilung wurden nach dem Prinzip der universellen Kalibrierung mithilfe der in Abschnitt 5.4 bestimmten Mark-Houwink-Koeffizienten in absolute Verteilungen umgerechnet. Zur Überprüfung von möglichen Nebenreaktionen wurden ESR-Untersuchungen durchgeführt. 


\subsection{Das ESR-Spektrum}

Zur Überprüfung von möglichen Nebenreaktionen, welche die Validität von PLP-SEC-Messungen einschränken würden, wurde die Temperaturabhängigkeit der ESR-Spektren von FA-HPBCHC untersucht. Wie in Abschnitt 7.4 gezeigt, sind Nebenreaktionen wie backbiting in ESRSpektren durch eine auftretende Temperaturabhängigkeit erkennbar. Diese Tatsache entsteht aus der Abweichung zwischen den Aktivierungsenergien der Propagation und der Nebenreaktion, welches in eine Veränderung der Zusammensetzung der Radikalpopulation mündet. In Abbildung 8.1 sind die ESR Spektren bei $-20^{\circ} \mathrm{C}, 20^{\circ} \mathrm{C}$ und $60^{\circ} \mathrm{C}$ von FA-HPBCHC dargestellt.

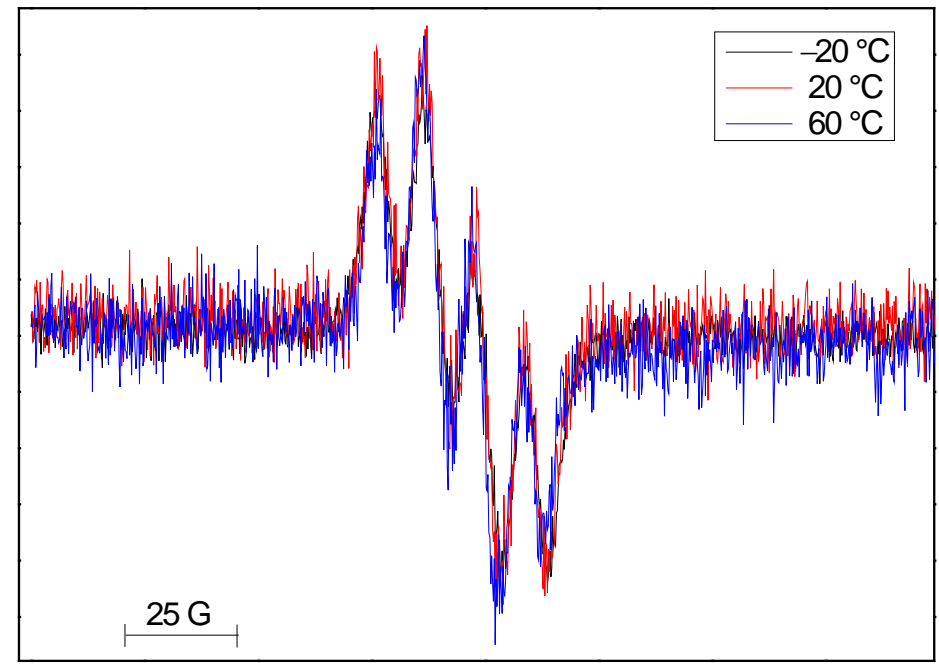

Abbildung 8.1: Normierte ESR-Spektren von FA-HPBCHC bei $-20^{\circ} \mathrm{C}, 20^{\circ} \mathrm{C}$ und $60^{\circ} \mathrm{C}$ in Toluol (20 wt\%). Aufgenommen wurden die Spektren mit einer Modulationsamplitude von 3 G und einer Scanzeit von 10 s. Die Initiierung erfolgte durch kontinuierliche Laserpulsinitiierung mit einer Pulsfrequenz von $20 \mathrm{~Hz}$ des Initiators Darocure $1173\left(50 \mathrm{mmol} \mathrm{L}^{-1}\right)$.

$\mathrm{Zu}$ beobachten ist ein vier Banden Spektrum, welches für jede der untersuchten Temperaturen identisch ist. Dementsprechend liegt kein Hinweis auf eventuelle Nebenreaktionen vor. 
Die Zuweisung von Kopplungsmustern und Konstanten in den erhaltenen Spektren ist nicht zweifelsfrei durchführbar. Unter der Annahme der Kopplung des Radikals mit dem $\alpha$-Fluoratom und zwei äquivalenter $\beta$ Wasserstoffatomen lässt sich das experimentelle Spektrum näherungsweise simulieren. Dazu wurden die Hyperfeinkopplungskonstanten $a(\alpha-\mathrm{F})=$ $1,33 \mathrm{mT}$ und $a(\beta-\mathrm{H})=0,92 \mathrm{mT}$ verwendet. Das Ergebnis ist in Abbildung 8.2 dargestellt.

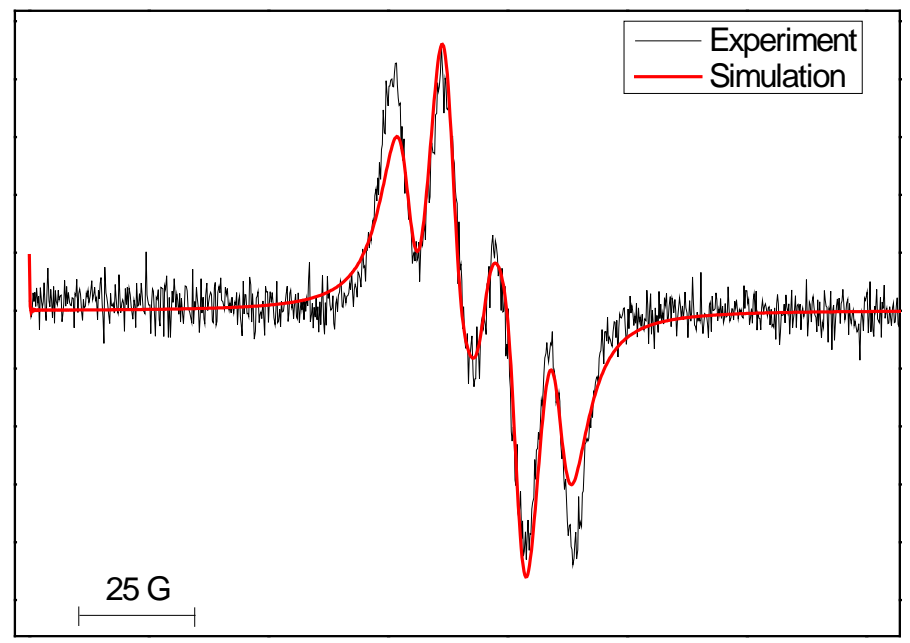

Abbildung 8.2: Schwarz: Experimentelles ESR-Spektrum von FA-HPBCHC in Toluol (20 wt \%) bei $-20^{\circ} \mathrm{C}$. Die Initiierung erfolgte durch kontinuierliche Laserpulsinitiierung mit einer Pulsfrequenz von $20 \mathrm{~Hz}$ des Initiators Darocure 1173 (50 $\left.\mathrm{mmol} \mathrm{L}^{-1}\right)$. Rot: Simuliertes Spektrum mit Hyperfeinkopplungskonstanten 1,33 mT und 0,92 mT.

Die Simulation erfordert die Annahme einer hohen Bandenbreite, eine Tatsache die durch den sehr hohen Propagationskoeffizienten der Substanz erklärt werden kann (siehe Abschnitt 8.4). Die dadurch entstehenden langen Polymerketten unterliegen vermutlich einer hohen Rotationshinderung welche in einer Bandenverbreiterung resultiert. Die ermittelten Kopplungskonstanten sind dabei niedriger als erwartet. Im Vergleich zu den Kopplungskonstanten von monofluorierten Methylradikalen ist $a(\alpha-\mathrm{F})$ um den Faktor drei geringer. ${ }^{[97]}$ Dies spricht deutlich für eine abweichende Geometrie des propagierenden Radikals von FA-HPBCHC 
und kann durch die großen strukturellen Unterschiede der restlichen Substituenten erklärt werden. ${ }^{[98]}$

\subsection{Strukturanalyse der PLP-SEC Kurven}

Jede Probe wurde zweimal durch SEC charakterisiert, um ein Maß für die Abweichung der SEC-Analyse zu ermitteln. Gemäß der IUPAC-Richtlinien wurden die Messwerte durch Variation der Pulsfrequenz und Initiatorkonzentration verifiziert. ${ }^{[54]}$ Die PLP-Experimente wurden in einem Temperaturbereich zwischen $20^{\circ} \mathrm{C}$ und $80{ }^{\circ} \mathrm{C}$ durchgeführt. Die Pulsanzahl wurde derart eingestellt, dass ein maximaler Umsatz von $5 \%$ erreicht wurde. Somit konnte einerseits genug Polymermaterial zur SEC-Analyse erhalten werden und andererseits von einer konstanten Monomerkonzentration ausgegangen werden.

In Abbildung 8.3 sind zwei exemplarische PLP-SEC-Kurven dargestellt, welche bei $20{ }^{\circ} \mathrm{C}$ aufgenommen wurden. Graph A entspricht $v_{\text {rep }}=25 \mathrm{~Hz}$ und Graph B $v_{\text {rep }}=40 \mathrm{~Hz}$ bei gleicher Initiatorkonzentration. Zu sehen sind bis zu drei POIs in jedem Chromatogramm. Die Struktur der Chromatogramme ist stark von der angewendeten $v_{\text {rep }}$ abhängig. Graph A entspricht dem ITC, in dessen Fall akkurate Ergebnisse für $k_{\mathrm{p}}$ aus den POIs entspringen. ${ }^{[72]}$ Graph B dagegen zeigt eine Struktur die sich in Richtung des LTRL annähert, erkennbar durch einen relativen Intensitätszuwachs im Bereich der Wendepunkte bei höheren Molmassen. ${ }^{[72]}$ Hier muss durch Vergleich von $M_{1} / M_{2}$ überprüft werden, dass der Grenzfall nicht überschritten wurde. 

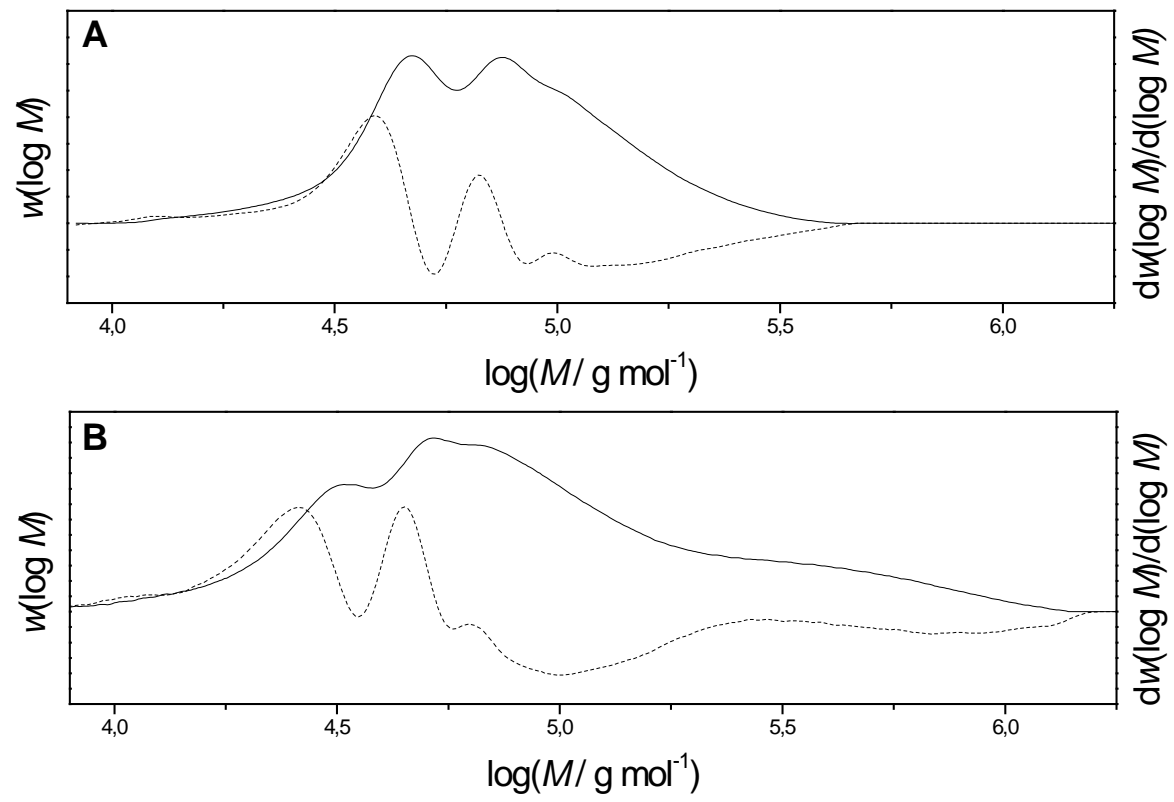

Abbildung 8.3: Molmassenverteilung (durchgezogene Linie) gewonnen aus SEC für Poly(FA-HPBCHC) hergestellt durch PLP $\left(20^{\circ} \mathrm{C}, 20\right.$ wt $\%$ in Toluol, $15 \mathrm{mmol}$ DMPA). Die erste Ableitung ist dargestellt durch die gepunktete Linie. Graph A wurde mit $v_{\text {rep }}=25 \mathrm{~Hz}$ und Graph B mit $v_{\text {rep }}=40 \mathrm{~Hz}$ aufgenommen.

Die Pulsfrequenzabhängigkeit der PLP Strukturen wurde für jede Temperatur systematisch untersucht. Die Ergebnisse bei $59,7^{\circ} \mathrm{C}$ für eine feste Initiatorkonzentration (DMPA, $15 \mathrm{mmol} \mathrm{L}^{-1}$ ) sind in Abbildung 8.4 dargestellt. $\mathrm{Zu}$ beobachten ist, dass die Werte bei 100 und $110 \mathrm{~Hz}$ geringfügig niedriger sind, während der Quotient $M_{1} / M_{2}$ weitab vom theoretischen Wert von 0,5 liegt. Ab $120 \mathrm{~Hz}$ liegen alle Werte, die durch den ersten POI bestimmt wurden $\left(k_{\mathrm{p}}(\mathrm{POI}-1)\right)$, in einem Abweichungsbereich welcher der experimentellen Genauigkeit entspricht (unter $2 \%$ ). Jedoch erreicht erst beim Experiment mit einer Pulsfrequenz von $160 \mathrm{~Hz} M_{1} / M_{2}$ einen zufriedenstellen Wert von 0,52. Dies deutet auf eine hohe Terminierung in der niederviskosen Toluol Lösung, welche keine PLP Kontrolle bei längeren Radikallebensdauern erlaubt. Dementsprechend müssen hohe $v_{\text {rep }}$ verwendet werden, um verlässliche Ergebnisse für $k_{\mathrm{p}} \mathrm{zu}$ erhalten. Aus diesem Grund wurden bei diesen Untersuchungen die 
Plateuwerte von POI-1 zur $k_{\mathrm{p}}$ Bestimmung verwendet. Dies bedeutet im Fall der Messwerte bei $59,7^{\circ} \mathrm{C}$, die Berücksichtigung der Messwerte ab $120 \mathrm{~Hz}$. Höhere $v_{\text {rep }}$ als die hier angegebenen ergaben keine PLP Strukturen. Alle berücksichtigten PLP-SEC-Daten sind in Abschnitt 11 tabellarisch zusammengefasst.

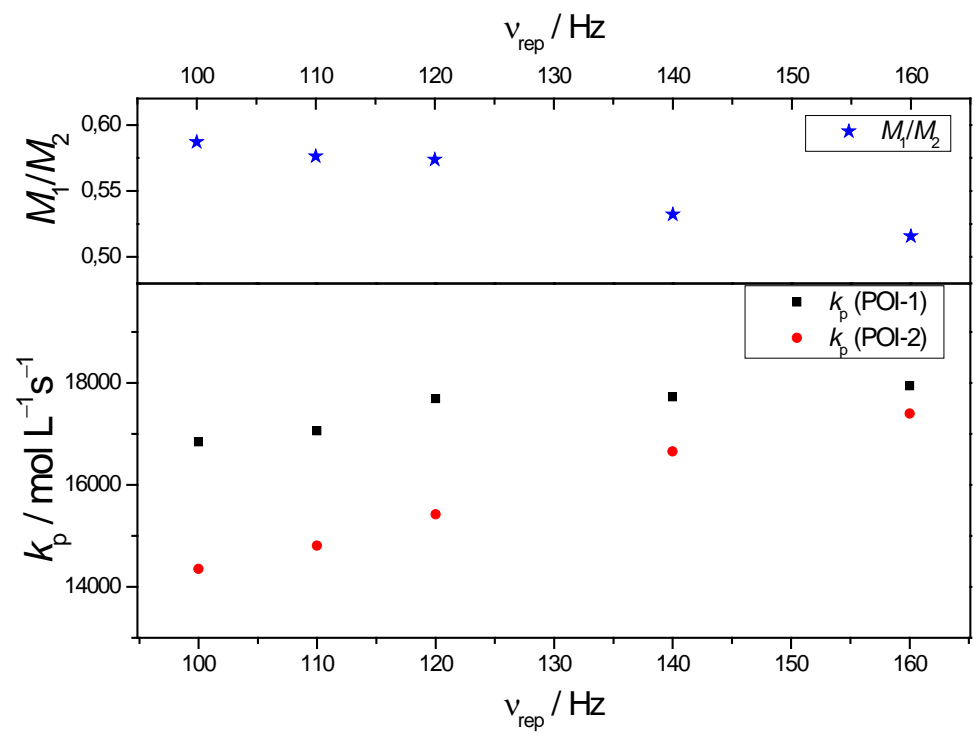

Abbildung 8.4: Ergebnisse der pulsfrequenzabhängigen PLP-SEC Experimente für FA-HPBCHC in Toluol bei $59,7^{\circ} \mathrm{C}(20 \mathrm{wt} \%, 15 \mathrm{mmol}$ DMPA). Im unteren Bereich sind die aus dem ersten Wendepunkt $\left(k_{\mathrm{p}}(\mathrm{POI}-1)\right)$ und dem zweiten Wendepunkt $\left(\boldsymbol{k}_{\mathrm{p}}(\mathrm{POI}-2)\right)$ erhaltenen $\boldsymbol{k}_{\mathrm{p}}$ Werte aufgetragen. Im oberen Bereich sind die entsprechenden Verhältnisse der Molmassen $M_{1} /$ $M_{2}$ gezeigt.

\subsection{Temperaturabhängigkeit von $\boldsymbol{k}_{\mathrm{p}}$}

Die Temperaturabhängigkeit von $k_{\mathrm{p}}$ wurde in dem Bereich von $20^{\circ} \mathrm{C}$ bis $80^{\circ} \mathrm{C}$ untersucht. Die Ergebnisse wurden in einer Auftragung nach Arrhenius linearisiert, wie in Abbildung 8.5 dargestellt. 


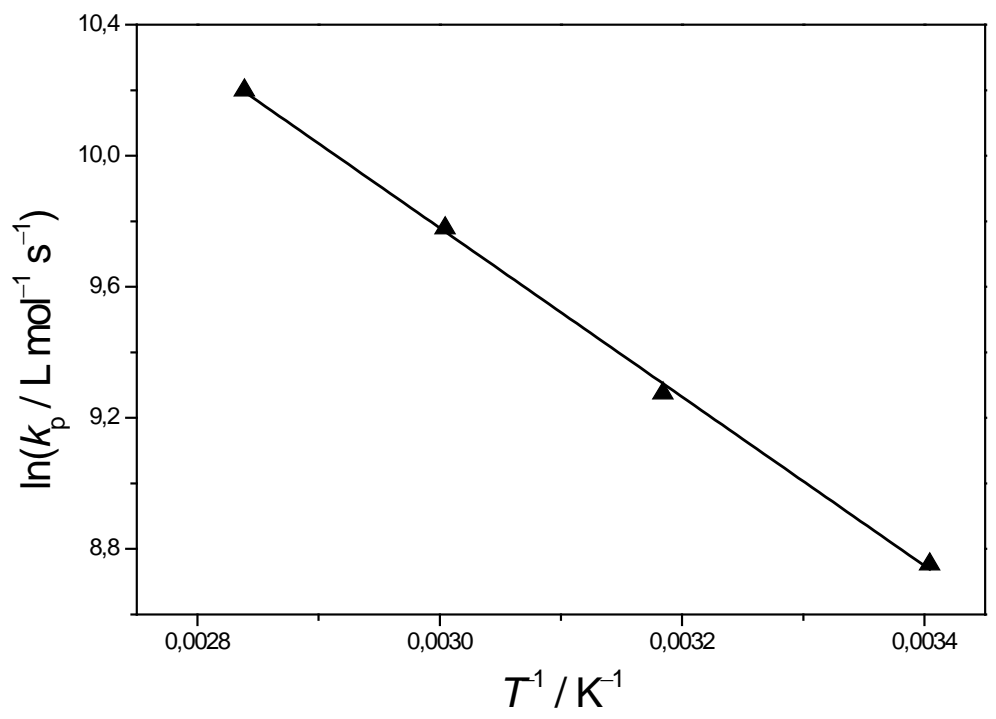

Abbildung 8.5: Arrhenius-Plot des Propagationskoeffizienten von FA-HРBCHC.

Die Temperaturabhängigkeit von $k_{\mathrm{p}}$ ergibt sich zu folgendem Zusammenhang.

$$
\ln \left(k_{\mathrm{p}} / \mathrm{L} \mathrm{mol}^{-1} \mathrm{~s}^{-1}\right)=17,51-2578 /(T / \mathrm{K})
$$

Daraus lässt sich eine Aktivierungsenergie von 21,4 $\mathrm{kJ} \mathrm{mol}^{-1}$ und ein präexponentieller Faktor von $4,02 \cdot 10^{7} \mathrm{~L} \mathrm{~mol}^{-1} \mathrm{~s}^{-1}$ ableiten. Hierbei kennzeichnet $T$ die absolute Temperatur. Der Fehler der Messungen kann mittels eines $95 \%$ Konfidenzintervalls ermittelt werden. Diese Abschätzung wurde durch die Annahme eines konstanten relativen Fehlers und der Anpassung durch die Methode der kleinsten Quadrate durchgeführt, wie von van Herk vorgeschlagen. ${ }^{[73]}$ Das Ergebnis dieser Analyse ist in Abbildung 8.6 dargestellt. 


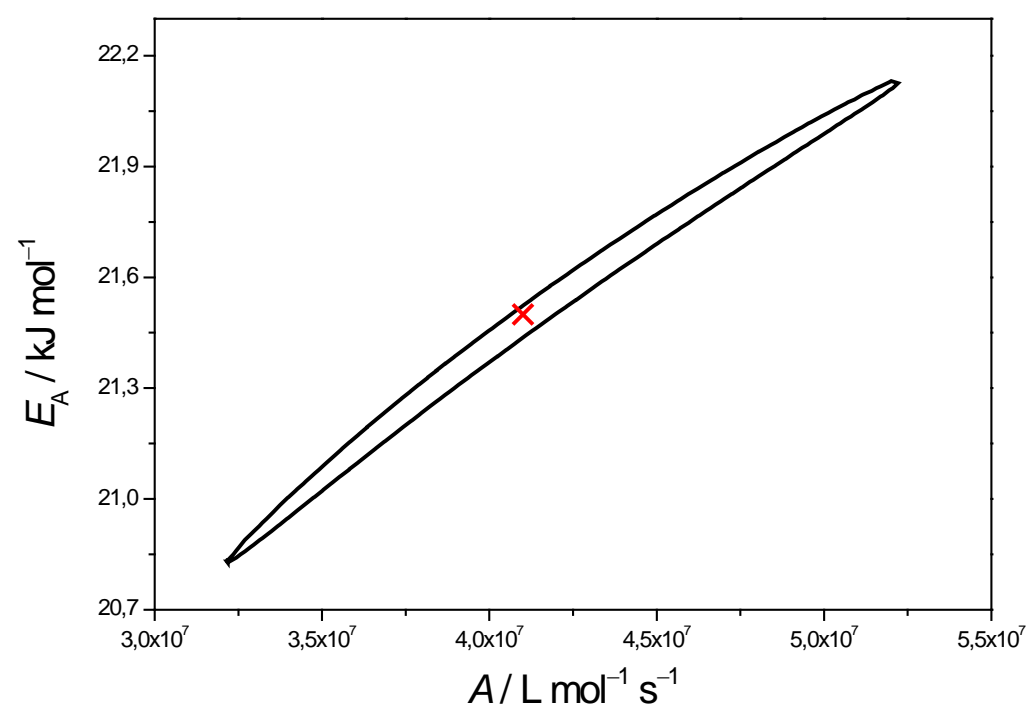

Abbildung 8.6: 95 \% Konfidenzintervall Region der Arrhenius Parameter des Propagationskoeffizienten von FA-HPBCHC. Das rote Kreuz gibt das Wertepaar mit der höchsten Wahrscheinlichkeit an.

Es ergibt sich für die Aktivierungsenergie ein Fehlerintervall von $20,8<E_{\mathrm{A}}<22,1 \mathrm{~kJ} \mathrm{~mol}^{-1}$ und für den präexponentiellen Faktor $3,1 \cdot 10^{7}<A<5,2 \cdot 10^{7} \mathrm{~L} \mathrm{~mol}^{-1} \mathrm{~s}^{-1}$.

FA-HPBCHC zeigt eine besonders schnelle Propagation. Interessant ist der Wert der Aktivierungsenergie welcher vergleichbar mit der von Methacrylaten und Vinylacetat ist. ${ }^{[74,99]}$ Aufgrund seines hohen präexponentiellen Faktors ist $k_{\mathrm{p}}$ von FA-HPBCHC jedoch deutlich höher als bei Methacrylaten. Wegen seiner geringen Größe kann ein ähnlicher sterischer Effekt des Fluorsubstituenten im Vergleich zu Acrylaten erwartet werden. Ueda et al. postulierten zusätzlich bei Fluoroacrylaten die Abwesenheit einer Resonanzstabilisierung auf das Radikalzentrum. ${ }^{[100]}$ Dies führt in der Schlussfolgerung zu einem wenig stabilisiertem propagierenden Radikal, welches eine hohe Neigung zur Polymerisation zeigen müsste, wie durch die hohen $k_{\mathrm{p}}$ Werte hier bestätigt. Im Vergleich zu dem einzigen bislang untersuchten Fluoroacrylat zeigt FA-HPBCHC deutlich höhere $k_{\mathrm{p}}$ Werte bei $\quad 30^{\circ} \mathrm{C} \quad\left(k_{\mathrm{p}}(\mathrm{EFA})=1120 \mathrm{~L} \mathrm{~mol}^{-1} \mathrm{~s}^{-1}\right.$, $k_{\mathrm{p}}($ FA-HPBCHC $\left.)=8150 \mathrm{~L} \mathrm{~mol}^{-1} \mathrm{~s}^{-1}\right) \cdot{ }^{[96]}$ Gründe hierfür könnte die 
geringere Verlässlichkeit der von Yamada et al. verwendeten RotatingSector-Methode im Vergleich zu PLP-SEC, starke Seitengruppen- oder auftretende Lösungsmitteleffekte sein.

In diesem Kapitel wurde die Bestimmung der Propagationskoeffizienten von FA-HPBCHC mittels PLP-SEC beschrieben. Die Methode erwies sich als geeignet zur Untersuchung dieser Monomerklasse. Jeder ermittelte Propagationskoeffizient wurde durch Variation der Pulsfrequenz und Initiatorkonzentration validiert. Bei den hier vorgestellten Untersuchungen handelt es sich, nach Wissen des Autors, um die erste Studie zur Propagation eines Fluoroacrylats mittels der genannten Methode. Eine Erklärung für den Ursprung der ungewöhnlich hohen Propagationsraten von FA-HPBCHC bleibt eine interessante Fragestellung und kann nur durch weitere Untersuchungen dieser Monomerfamilie geklärt werden. 


\section{Propagation von EA-HPBCHC}

\subsection{Einleitung}

Im Folgenden wird die Bestimmung des Propagationkoeffizienten von EA-HPBCHC, dessen Struktur in Abbildung 9.1 dargestellt ist, beschrieben. Im Allgemeinen sind Ethacrylate aufgrund ihrer geringen Neigung zur Polymerisation in der Vergangenheit wenig untersucht worden. Der Propagationskoeffizient von Methylethacrylat wurde von Kobatake und Yamada mittels ESR Spektroskopie bestimmt und beträgt bei $60{ }^{\circ} \mathrm{C} 8,6 \mathrm{~L} \mathrm{~mol}^{-1} \mathrm{~s}^{-1}$. [101] Somit ist der Wert für Methylethacrylat ca. 100 mal kleiner als $k_{\mathrm{p}}$ von Methylmethacrylat $\left(834 \mathrm{~L} \mathrm{~mol}^{-1} \mathrm{~s}^{-1}\right){ }^{[5]}$ Weiterhin gibt es thermodynamische Nachteile bei der Polymerisation dieser Substanzklasse. Penelle et al. untersuchten die Thermodynamik der Polymerisation von Methylethacrylat in Substanz und fanden für dieses Monomer eine Ceiling-Temperatur von $82{ }^{\circ} \mathrm{C} .{ }^{[102]}$ Die Ceiling-Temperatur bezeichnet die Temperatur bei der Polymerisation und Depolymerisation im dynamischen Gleichgewicht sind. Dementsprechend findet ab dieser Temperatur kein Umsatz mehr statt. Es ist stark davon auszugehen, dass dieser Effekt aufgrund der starken sterischen Hinderung, verursacht durch die $\alpha$-Ethylgruppe, zustande kommt. Dies resultiert in einer geringen Reaktionsenthalpie, die nach der Definition der freien Reaktionsenthalpie nach Gibbs schon bei niedrigen Temperaturen durch den Entropieterm ausgeglichen werden kann. 


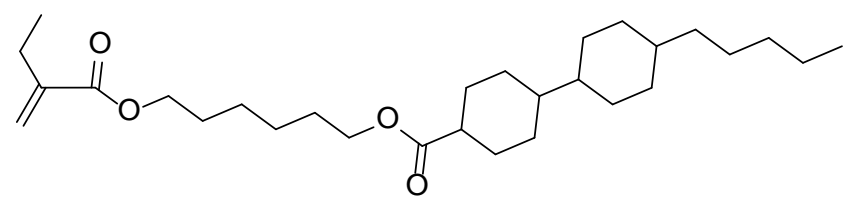

\section{EA-HPBCHC}

\section{Abbildung 9.1: Strukturformel von EA-HPBCHC.}

Aus den zuvor genannten Gründen wurde bei dieser Untersuchung nur bis zu geringen Umsätzen (unter $1 \%$ ) polymerisiert. Des Weiteren fanden alle Experimente in Substanz und bei $60{ }^{\circ} \mathrm{C}$ statt, um einen Einfluss der Depolymerisation auf die Bestimmung des Propagationskoeffizienten zu verhindern. Die sehr niedrigen Propagationskoeffizienten und durchschnittlichen Terminierungskoeffizienten welche für EA-HPBCHC zu erwarten sind und die niedrigen molaren Konzentrationen, sich ergebend aus der hohen molaren Masse von EA-HPBCHC, machen dieses System ungeeignet zur Untersuchung mittels PLP-SEC. Aufgrund dessen wurde ein alternatives Verfahren zur Bestimmung von $k_{\mathrm{p}}$ verwendet.

Eine Methode zur Bestimmung von Propagationskoeffizienten ist die ESRSpektroskopie in Verknüpfung mit Umsatzmessungen. ${ }^{[103]}$ Im Vergleich zu der durch das IUPAC-Komitee empfohlenen PLP-SEC Methode weist diese Methode einige zusätzliche experimentellen Schwierigkeiten auf. ${ }^{[104]}$ Besondere Vorsicht muss zum einen bei der Integration der Spektren walten. Eine Grundlinienkorrektur ist zwingend erforderlich, um genaue Radikalkonzentrationen zu bestimmen. Messparameter wie Reaktionszeit und Radikalkonzentration müssen variiert werden, um konsistente Datensätze zu erkennen. Das Vorhandensein von dem quasistationären Zustand muss durch zeitabhängige Messungen überprüft werden. Es muss überprüft werden, dass die Messparameter in dem verwendeten Konzentrationsbereich keine Saturation der Signalintensität erzeugen. Die Kalibrierung des Spektrometers muss durch Lösungen erfolgen, die möglichst genau in der Zusammensetzung den Messlösungen entsprechen und ähnliche Radikalkonzentrationen wählen. 


\subsection{Das ESR-Spektrum}

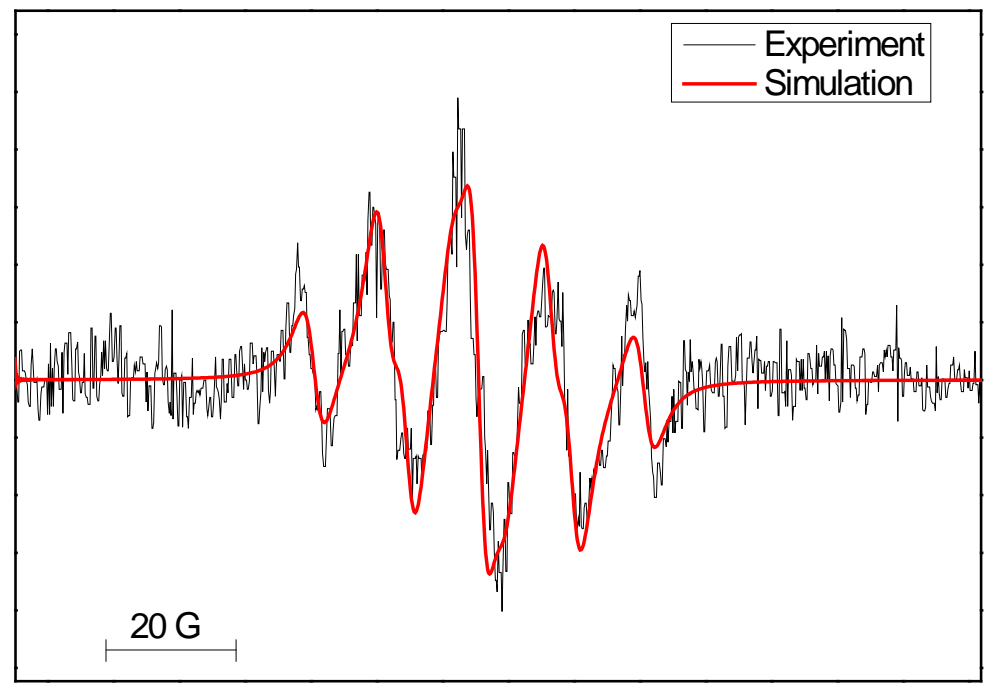

Abbildung 9.2: Schwarz: Experimentelles ESR-Spektrum von EA-HPBCHC bei $60{ }^{\circ} \mathrm{C}$. Aufgenommen mit einer Modulationsamplitude von $1 \mathrm{G}$ und einer Scanzeit von $10 \mathrm{~s}$, wurden 10 Scans gemittelt. Die Initiierung erfolgte thermisch durch Zugabe von Lauroylperoxid. Rot: Simuliertes Spektrum mit den Kopplungskonstanten 1,41 mT und 1,10 mT.

Das experimentelle und das simulierte Spektrum von EA-HPBCHC sind in Abbildung 9.2 dargestellt. Das experimentelle Spektrum gleicht den von Kobatake und Yamada für Methylethacrylat und Cyclohexylethacrylat aufgenommenen Spektren. ${ }^{[101]}$ Hierbei handelt es sich um ein fünf Banden Spektrum mit hoher Peakbreite. Das simulierte Spektrum wurde durch Annahme der Hyperfeinkopplung mit zwei äquivalenten $\beta$ Methylenwasserstoffatomen mit den Kopplungskonstanten 1,41 mT und $1,10 \mathrm{mT}$ simuliert. Interessanterweise entsprechen die verwendeten Kopplungskonstanten exakt den Kopplungskonstanten, die von Kamachi dem propagierendem Radikal von Itaconsäuredibutylester zugeordnet wurden. ${ }^{[105]}$ Beide Spezies ähneln sich in ihrer unmittelbaren Umgebung um das Radikalzentrum. Hierbei ist, wie ausführlich schon für Methacrylate beobachtet wurde, von einer gehinderten Rotation entlang des Polymerrückgrats auszugehen. ${ }^{[89,106]}$ Dies führt zur Fixierung des Winkels eines Methylenwasserstoffpaares in Bezug zu dem einfach besetzten p- 
Orbital, während das Methylenwasserstoffpaar an der Ethylengruppe freie Rotation erfährt und somit eine gemittelte Kopplungskonstante aufweist. Dies führt zu einer Beeinflussung des Spektrums nach Gleichung (7.4).

\subsection{Bestimmung von $k_{\mathrm{p}}$ und Abschätzung von $k_{\mathrm{t}}$}

Die Bestimmung des Propagationskoeffizienten basiert auf der Verwendung des integrierten Zeitgesetzes (9.1). ${ }^{[107]}$

$$
\ln \frac{1}{1-X}=k_{\mathrm{P}} \cdot \int c_{\mathrm{R}}(t) d t
$$

Zur Bestimmung von $k_{\mathrm{p}}$ wurde durch Zugabe eines thermischen Initiators eine Polymerisation unter quasistationären Bedingungen durchgeführt. Identische Messlösungen wurden verwendet, um einerseits die Radikalverläufe im ESR zu messen und andererseits den Umsatz durch gravimetrische Analyse zu bestimmen. Für letzteres wurde die Reaktion in einem Polymerisationsgläschen erhitzt, durch Zugabe von einer inhibierenden Hydrochinon Lösung in Toluol gestoppt und in Ethanol gefällt. Nach Trocknung unter Vakuum wurde der Umsatz gravimetrisch bestimmt. Zur Bestimmung absoluter Radikalkonzentrationen wurde das Spektrometer wie in Abschnitt 4.6 ausführlich beschrieben kalibriert. Die Kalibrierlösungen wurden durch Zugabe vom stabilen Radikal TEMPO zu der Monomerlösung hergestellt. Folglich wurden bei allen ESR-Messungen ein Röhrchendurchmesser von 5 mm und ein Probenvolumen von $200 \mu \mathrm{L}$ eingehalten. Zur Beeinflussung der Radikalkonzentration wurde die Initiatorkonzentration in einem Bereich von $19 \mathrm{mmol}$ bis $145 \mathrm{mmol}$ variiert. In Tabelle 9.1 sind die Ergebnisse der Untersuchungen zusammengefasst. Quasistationäre Bedingungen wurden durch konstante Signalhöhe des ESR-Spektrometers festgestellt. Die Propagationskoeffizienten wurden, wie zuvor beschrieben, nach Gleichung (9.1) berechnet. Aus den durchgeführten Untersuchungen ergibt sich die Möglichkeit der Bestimmung eines kettenlängengemittelten 
Terminierungskoeffizienten $\left\langle k_{\mathrm{t}}\right\rangle$. Unter Annahme von quasistationären Bedingungen und der Vernachlässigung von Primärradikalterminierung lässt sich $\left\langle k_{\mathrm{t}}\right\rangle$ aus Gleichung (3.5) bestimmen. Dazu wurde die Zerfallskonstante vom Initiator Lauroylperoxid in Benzol aus der Literatur verwendet $\left(k_{\mathrm{d}}=9,2 \cdot 10^{-6} \mathrm{~s}^{-1}\right){ }^{[70]}$ Weiterhin wurde die gleiche Initiatoreffizienz von Lauroylperoxid wie bei der Polymerisation von Styrol angenommen $(f=0,36) .{ }^{[108]}$ Der Verlust an Initiatorkonzentration bis zur längsten Messzeit beträgt $4 \%$ und ist im Rahmen der gegebenen experimentellen Ungenauigkeit zu vernachlässigen.

Tabelle 9.1: Übersicht der experimentellen Ergebnisse der thermisch initiierten Polymerisation von EA-HPBCHC in Substanz durch den Initiator Lauroylperoxid bei $60^{\circ} \mathrm{C}$.

\begin{tabular}{cccccc}
\hline $\begin{array}{c}c_{\mathrm{I}} / \\
\mathrm{mol} \mathrm{L}^{-1}\end{array}$ & $\begin{array}{c}c_{\mathrm{r}} \cdot 10^{7} / \\
\mathrm{mol} \mathrm{L}^{-1}\end{array}$ & $\begin{array}{c}t / \\
\mathrm{s}\end{array}$ & $\begin{array}{c}X / \\
\%\end{array}$ & $\begin{array}{c}k_{\mathrm{p}} / \\
\mathrm{L} \mathrm{mol}^{-1} \mathrm{~s}^{-1}\end{array}$ & $\begin{array}{c}\left\langle k_{\mathrm{t}}\right\rangle \cdot 10^{-6} / \\
\mathrm{Lol}^{-1} \mathrm{~s}^{-1}\end{array}$ \\
\hline 0,144 & 3,49 & 3900 & 0,72 & 5,3 & 3,9 \\
0,082 & 3,02 & 3000 & 0,37 & 4,0 & 3,0 \\
0,055 & 2,53 & 2700 & 0,27 & 4,0 & 2,9 \\
0,034 & 2,06 & 4200 & 0,24 & 2,8 & 2,7 \\
0,019 & 1,88 & 3600 & 0,48 & 7,1 & 1,8 \\
\hline
\end{tabular}

Durch Mittelwertbildung konnten also die Koeffizienten $k_{\mathrm{p}}\left(60{ }^{\circ} \mathrm{C}\right)=4,6 \pm 1,6 \mathrm{~L} \mathrm{~mol}^{-1} \mathrm{~s}^{-1}$ und $\left\langle k_{\mathrm{t}}\right\rangle\left(60^{\circ} \mathrm{C}\right)=2,9 \pm 0,8 \cdot 10^{6} \mathrm{~L} \mathrm{~mol}^{-1} \mathrm{~s}^{-1}$ bestimmt werden.

Der Propagationskoeffizient liegt nahe denen für andere Ethacrylaten bestimmten Werten. Die schon zuvor erwähnte Studie von Kobatake und Yamada ergab für Methylethacrylat und Cyclohexylethacrylat ein $k_{\mathrm{p}}$ bei $60{ }^{\circ} \mathrm{C}$ von 8,6 $\mathrm{L} \mathrm{mol}^{-1} \mathrm{~s}^{-1}$ und 1,6 $\mathrm{L} \mathrm{mol}^{-1} \mathrm{~s}^{-1}$. 101] Eine Korrelation zwischen Struktur der Seitenkette und Propagationsgeschwindigkeit erscheint wegen der geringen Datenmenge und der starken Fehleranfälligkeit der ESR-Methode nicht sinnvoll. Welchen Einfluss die 
eingeführte Sterik auf Aktivierungsenergie und präexponentiellen Faktor dieser Substanzklasse zeigt, ist nicht mit dieser Methode nachzuweisen. Höhere Temperaturen als $60^{\circ} \mathrm{C}$ sind aufgrund des Einflusses der Depolymerisation nicht sinnvoll. Messungen bei niedrigeren Temperaturen sind wegen der extrem niedrigen Rate, der Fehleranfälligkeit durch Verwendung von Photoinitiatoren (Nebenprodukterzeugung) und der nicht ausreichenden Genauigkeit der Methode nicht zielführend.

Für den Terminierungskoeffizienten ist ein deutlicher Einfluss der Seitenkette zu beobachten. Die von Kobatake und Yamada ermittelten Werte von $\left\langle k_{\mathrm{t}}\right\rangle\left(60^{\circ} \mathrm{C}\right)$ für Methylethacrylat und Cyclohexylethacrylat liegen bei $21 \cdot 10^{6}$ und $1,8 \cdot 10^{6} \mathrm{~L} \mathrm{~mol}^{-1} \mathrm{~s}^{-1}$. ${ }^{[101]}$ Wie in Abschnitt 3.4.2 diskutiert ist die Terminierung ein diffusionskontrollierter Prozess. Dementsprechend hat die Viskosität der Lösung einen ausschlaggebenden Einfluss. Kobatake und Yamada erwähnen eine optisch feststellbare höhere Viskosität von Cyclohexylethacrylat im Vergleich zu Methylethacrylat, ohne diese Beobachtung durch Messdaten zu stützen. ${ }^{[101]}$ Da keine Daten zur Viskosität der Ethacrylatfamilie in Substanz vorliegen, kann nur gemutmaßt werden, dass es im Falle von Cyclohexylethacrylat und EA-HPBCHC durch intermolekulare Wechselwirkungen zu einer Erhöhung der Viskosität kommt. Weiterhin kann in Tabelle 9.1 eine Tendenz zu größeren $\left\langle k_{\mathrm{t}}\right\rangle$-Werten bei höheren Radikalkonzentrationen beobachtet werden. Dies ist eindeutig der Kettenlängenabhängigkeit der Terminierungskoeffizienten geschuldet, die ausführlich in Abschnitt 3.4.2. beschrieben ist. Wird nun die kinetische Kettenlänge $v$ der Makromoleküle für jeden Reaktionsansatz aus dem Verhältnis der Propagationsgeschwindigkeit zur Terminierungsgeschwindigkeit errechnet und werden die berechneten $\left\langle k_{\mathrm{t}}\right\rangle$ Werten gegen $v$ nach (3.18) doppeltlogarithmisch auftragen, lässt sich ein linearer Zusammenhang erahnen (siehe Abbildung 9.3).

Aus dieser Auftragung kann eine Schätzung für die Koeffizienten im Kurzkettenbereich getätigt werden. Es ergibt sich $k_{\mathrm{t}}(1,1)=1,1 \cdot 10^{7} \mathrm{~L} \mathrm{~mol}^{-1} \mathrm{~s}^{-1}$ und $\alpha_{\mathrm{s}}=0,52$. Eine Berücksichtigung des Langkettenbereichs ist aufgrund der sehr kurzen kinetischen Kettenlänge (7 bis 29) zu vernachlässigen. Weiterhin muss diese Auftragung als grobe Näherung aufgrund der Vereinfachung $v=i$ betrachtet werden. $\mathrm{Zu}$ beobachten ist, dass der Einfluss der Kettenlängenabhängigkeit der 
Terminierung bei kontinuierlichen Messungen für dieses System schon bei mäßiger Radikalkonzentrationsvariation sichtbar wird und dies zur Schätzung dieses Phänomens verwendet werden kann.

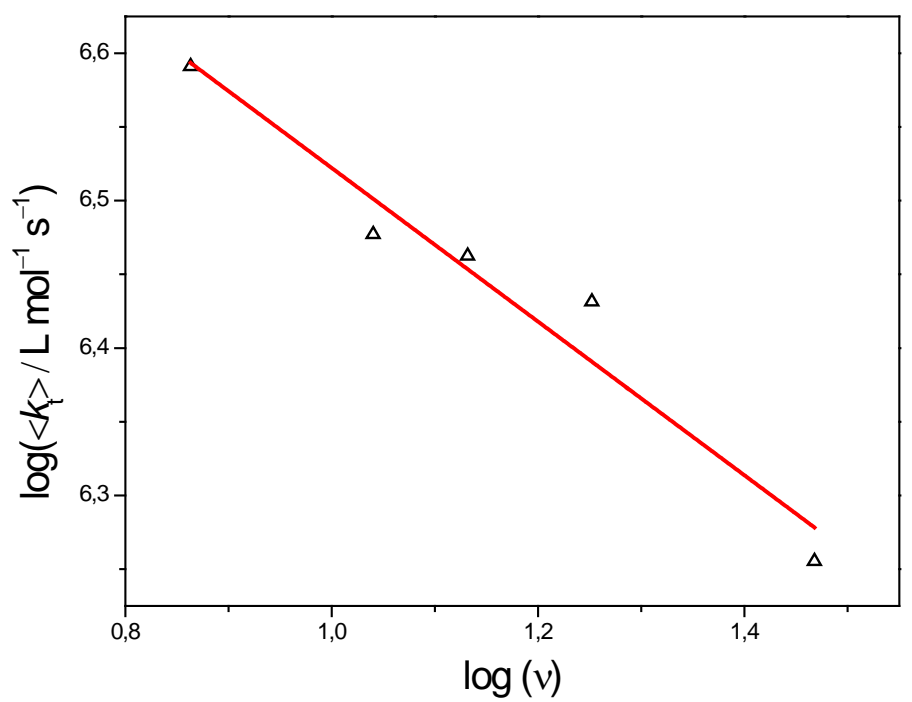

Abbildung 9.3: Doppeltlogarithmische Auftragung nach dem composite model zur Abschätzung der Terminierungsparameter im Kurzkettenbereich.

Abschließend lässt sich sagen, dass der Propagationskoeffizient von EA-HPBCHC bei $60^{\circ} \mathrm{C}$ in der gleichen Größenordnung anderer Ethacrylate liegt. Die Terminierung zeigt eine Kettenlängenabhängigkeit und kann durch quasistationäre Experimente in Verbindung mit ESR abgeschätzt werden. Somit sind die wichtigsten kinetischen Parameter zur Beschreibung der Polymerisation von EA-HPBCHC bestimmt worden und ein Beitrag zum Verständnis der Kinetik der radikalischen Polymerisation von Ethacrylaten geleistet worden. 


\section{Terminierung von Methacrylaten in nematischer und chiral- nematischer Phase}

\subsection{Einleitung}

Zur vollständigen kinetischen Beschreibung einer Polymerisation ist das Verständnis der Terminierungskinetik von größter Bedeutung. Hierbei hat sich SP-PLP-ESR aufgrund der direkten Messung von Radikalkonzentration-Zeit-Verläufen als Schlüsselmethode herausgestellt. ${ }^{[109]}$ Nach Wissen des Autors ist bis zum jetzigen Zeitpunkt keine Messung von Terminierungskoeffizienten in flüssigkristalliner Lösung mit dieser oder einer Methode mit vergleichbarer Genauigkeit durchgeführt worden. Flüssigkristalline Lösungen sind von grundsätzlichem Interesse aufgrund der in der Vergangenheit berichteten ungewöhnlichen Entwicklung von Polymerisationsraten in smektischen Phasen mit der Temperatur. ${ }^{[25-27]}$ Dabei ergab sich der Effekt aus einer Bildung von erhöhten lokalen Konzentrationen an Monomer. ${ }^{[26]}$

Im Rahmen dieses Kapitels soll die kettenlängenabhängige Terminierung eines isotropen Methacrylats (Ethylhexylmethacrylat, EHMA) und eines mesogenen Methacrylats (MA-HPBCHC) in nematischer und chiralnematischer Lösung mittels SP-PLP-ESR untersucht werden. Als nematische Phase wurde das industriell verwendetes Gemisch BL-087 von 
der Firma Merck KGaA verwendet. Die chiralen Phasen wurden durch Zugabe von 5 wt\% und 7 wt\% des chiralen Dotierstoffes R-5011 der Firma Merck KGaA hergestellt. Der Monomeranteil betrug bei allen Messungen $10 \mathrm{wt} \%$. Der Umsatz aller in diesem Kapitel gezeigten Untersuchungen betrug maximal 5\%, um einen Einfluss der Umsatzabhängigkeit der Terminierung auszuschließen. Dies wurde nach Durchführung der SPPLP-ESR Experimente mittels FT-NIR überprüft. Die zur Bestimmung der composite model Parameter benötigten $k_{\mathrm{p}}$-Werte wurden für MA-HPBCHC in Abschnitt 6 bestimmt und für EHMA aus der Literatur entnommen. ${ }^{[110]}$

\subsection{Das ESR-Spektrum von Methacrylaten in nematischer und chiral-nematischer Phase}

Das ESR-Spektrum von Methacrylaten ist charakteristisch für diese Substanzklasse und besteht aus 13 Banden, mit fünf Banden höherer Intensität und acht inneren Banden. Dieses Spektrum kommt durch Kopplung des Radikals mit den $\beta$-Wasserstoffatomen und der Überlappung verschiedener Konformerspektren mit gehinderter Rotation zustande. ${ }^{[16]}$ In Abbildung 10.1 sind die ESR-Spektren von MA-HPBCHC bei $40^{\circ} \mathrm{C}$ und $100{ }^{\circ} \mathrm{C}$ in der nematischen Flüssigkeit BL-087 zu sehen. Zu beobachten ist eine starke Abhängigkeit der Intensität der inneren Banden von der Temperatur. Dieses Verhalten ist schon wiederholt für Methacrylate beobachtet worden und wird der abnehmenden Rotationshinderung mit steigender Temperatur zugesprochen. ${ }^{[90]}$ Anisotrope Effekte auf das Spektrum der verwendeten Methacrylate in nematischer und chiralnematischer Lösung konnten nicht beobachtet werden. Die mit einem Stern gekennzeichnete Bande wurde bei den in den Abschnitten 10.4 und 10.5 gezeigten zeitaufgelösten SP-PLP-ESR Messungen der Radikalkonzentration verwendet. Dadurch werden Überlappungen mit Initiatorfragmentradikale verhindert, dessen Spektren oftmals mit der mittleren Bande überlappen können. ${ }^{[111]}$ 


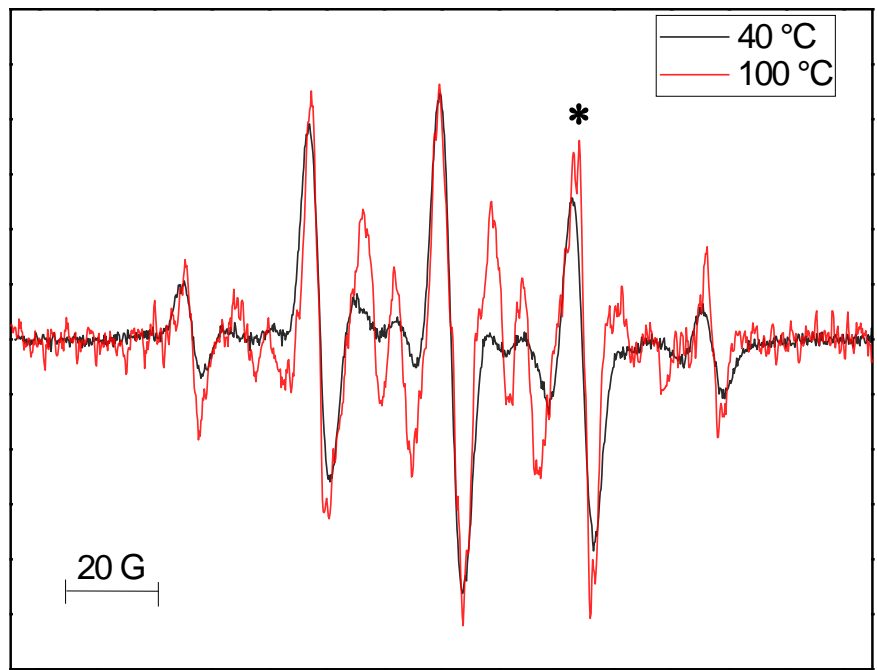

Abbildung 10.1: ESR-Spektren von MA-HPBCHC in der nematischen Flüssigkeit BL-087 bei $40{ }^{\circ} \mathrm{C}$ (Schwarz) und $100{ }^{\circ} \mathrm{C}$ (Rot). Die Spektren wurden mit einer Modulationsamplitude von $3 \mathrm{G}$ aufgenommen. Die Radikale wurden mittels kontinuierlicher Bestrahlung des Initiators Darocure ${ }^{\circledR} 1173$ $\left(0,1 \mathrm{~mol} \mathrm{~L}^{-1}\right)$ durch eine Quecksilberbogenlampe erzeugt. Die mit einem Stern gekennzeichnete Bande wurde für zeitaufgelösten SP-PLP-ESR Messungen verwendet.

\subsection{Ordnung der nematischen Phase im Magnetfeld des ESR-Spektrometers}

Nematische Phasen werden zur Erzeugung eines einheitlichen Direktors entlang von alignment layer ausgerichtet. Ziel der im Folgenden geschilderten Untersuchung war es zu bestimmen, ob das Magnetfeld im verwendeten ESR-Spektrometer zu einer makroskopischen Ausrichtung der mit Monomer versetzten Phase von BL-087 führt. Auf diese Weise kann auf eine Vergleichbarkeit mit durch alignment layer ausgerichteten Systemen geschlossen werden.

Dazu wurde die von Tanaka und Kuwata beobachtete Anisotropie der ESRSpektren von 4-Oxo-2,2,6,6-tetramethyl-1-piperidinyloxy (TEMPONE) in nematischen Lösungen angewendet. ${ }^{[112]}$. Dieses stabile Radikal ordnet sich durch Wechselwirkungen mit der nematischen Phase entlang des durch das Magnetfeld erzeugten Direktors teilweise an. ${ }^{[113]}$ Diese Anisotropie hat eine 
Auswirkung auf die Hyperfeinkopplungskonstanten $a(\mathrm{~N})$ des ESRSpektrums von TEMPONE. Die anisotropische Anordnung der gelösten TEMPONE Radikale kann mittels des Ordnungsparameters $\Phi_{\mathrm{zz}}$ quantifiziert werden. Dieser beschreibt den Anteil an ausgerichteten TEMPONE Molekülen. Dabei soll $\gamma$ den durchschnittlichen Winkel zwischen Molekülachse und Direktor der Phase angeben. Die Differenz der Kopplungskonstanten in nematischer und isotroper Phase im Verhältnis zum dipolaren Tensor des Moleküls, $A_{\mathrm{Nzz}}$, gibt näherungsweise $\Phi_{\mathrm{zz}}$ an. $^{[114]}$

$$
\frac{a^{\text {nem }}(\mathrm{N})-a^{\text {iso }}(\mathrm{N})}{A_{\mathrm{Nzz}}} \approx \Phi_{\mathrm{zz}}=\frac{3 \cos (\gamma)^{2}-1}{2}
$$

Die Spektren von TEMPONE in den Mischungen aus dem jeweiligen Monomer (EHMA und MA-HPBCHC, $10 \mathrm{wt} \%$ ) und BL-087 wurden für verschiedene Temperaturen in der nematischen und isotropen Phase aufgenommen. In Abbildung 10.2 sind die Spektren von TEMPONE in MA-HPBCHC und BL-087 bei $20^{\circ} \mathrm{C}$ (nematische Phase) und $100{ }^{\circ} \mathrm{C}$ (isotrope Phase) dargestellt. Zu sehen ist eine starke Abweichung von $a(\mathrm{~N})$.

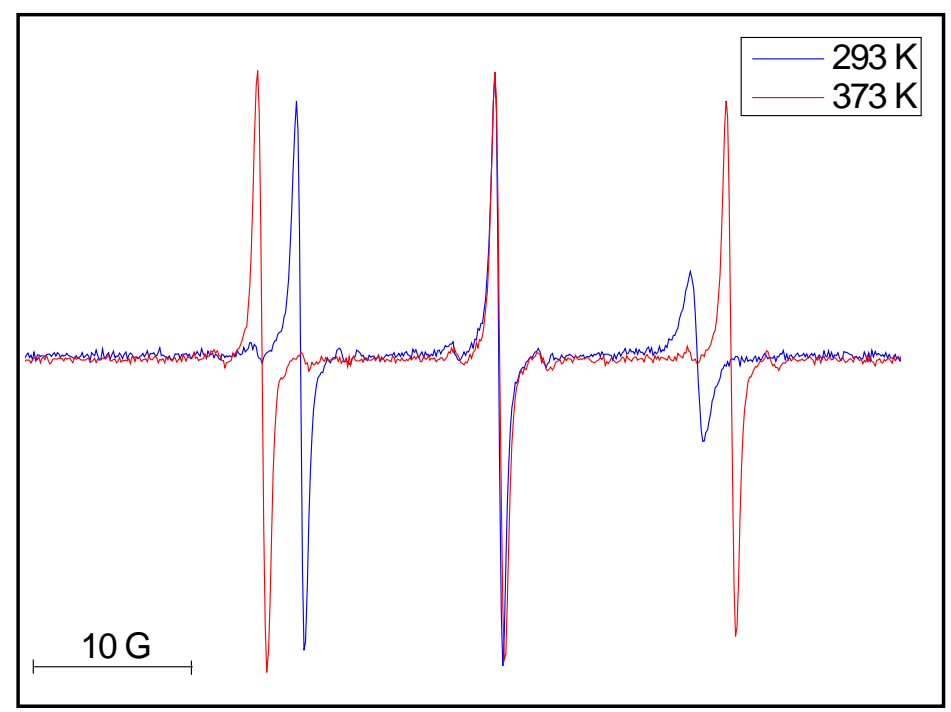

Abbildung 10.2: ESR-Spektren von TEMPONE in BL-087 und MA-HPBCHC (10 wt \%) bei $293 \mathrm{~K}$ (Blau) und $373 \mathrm{~K}$ (Rot). Die Spektren wurden mit einer Modulationsamplitude von $1 \mathrm{G}$ aufgenommen. 
Für $A_{\mathrm{Nzz}}$ wurde der von Griffith et al. ermittelte Wert verwendet $(16,4 \mathrm{G}) \cdot{ }^{[115]}$ Daraus konnte die Entwicklung von $\quad \Phi_{\mathrm{zz}}$ nach der Temperatur aus Gleichung (10.1) für EHMA und MA-HPBCHC in BL087 berechnet werden (siehe Abbildung 10.3).
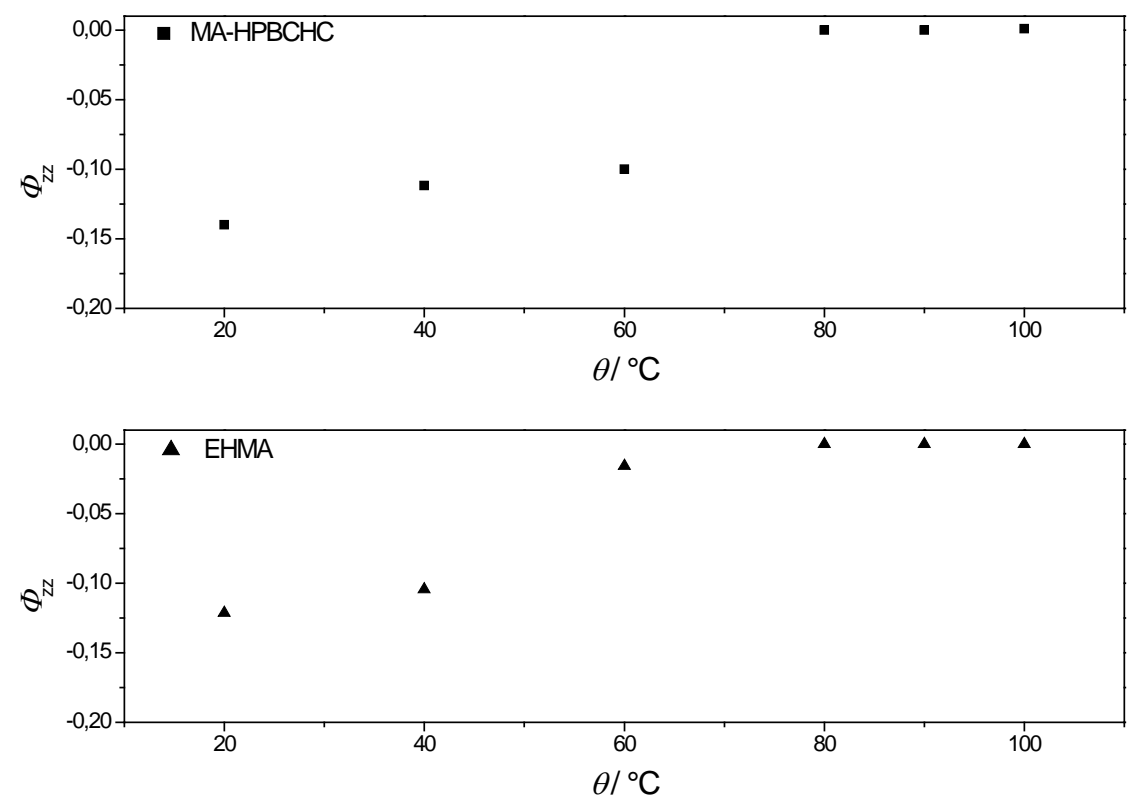

Abbildung 10.3: $\Phi_{\mathrm{zz}}$ gegen die Temperatur für Lösungen bestehend aus MA-HPBCHC (oben) bzw. EHMA (unten) und BL-087.

$\mathrm{Zu}$ beobachten ist ein negatives $\Phi_{\mathrm{zz}}$, welches sich aus der senkrechten Anordnung des einfach besetzten p-Orbitals von TEMPONE zum Magnetfeld des ESR-Spektrometers ergibt. Dies wurde für Spektren von TEMPONE in anderen nematischen Phasen beobachtet. ${ }^{[112]}$ Dementsprechend bedeutet das höchste Maß an Ordnung $\Phi_{\mathrm{zz}}=-0,5$. Die Auftragung zeigt deutlich, dass eine Ausrichtung der nematischen Phase im Magnetfeld des ESR-Spektrometers stattfindet. Nach dem Übertritt in die isotrope Phase verschwindet der anisotrope Effekt. Zu beachten ist, dass $\Phi_{\mathrm{zz}}$ ein Maß für die Ordnung der TEMPONE Moleküle angibt und nicht für die flüssigkristallinen Moleküle der nematischen Phase. Aufgrund der fehlenden Anisotropie von TEMPONE in reiner Lösung ist davon auszugehen, dass die flüssigkristallinen Bestandteile von BL-087 ein höheres Maß an Ordnung aufweisen. 


\subsection{Terminierung von Methacrylaten in nematischer Phase}

SP-PLP-ESR Messungen wurden für $10 \mathrm{wt} \%$ EHMA und MA-HPBCHC in der nematischen Flüssigkeit BL-087 durchgeführt. Hierzu wurden die composite model Parameter in einem Temperaturbereich zwischen $20^{\circ} \mathrm{C}$ und $100^{\circ} \mathrm{C}$ bestimmt. Der Klärpunkt von BL-087 liegt im reinen Gemisch bei $89^{\circ} \mathrm{C}$. Durch Zugabe von Monomer und Initiator wird der Klärpunkt der flüssigkristallinen Mischung herabgesetzt. Hierbei wurde durch Zugabe von EHMA der Klärpunkt der Mischung auf $58{ }^{\circ} \mathrm{C}$ und durch Zugabe von MA-HPBCHC auf $76^{\circ} \mathrm{C}$ herabgesetzt. Allen Mischungen wurde der Initiator Darocure 1173 in einer Konzentration von 50 mmol hinzugegeben, womit Anfangsradikalkonzentrationen $\left(\mathrm{c}_{\mathrm{R}}^{0}\right)$ von ca. $1 \cdot 10^{-5} \mathrm{~mol} \mathrm{~L}^{-1}$ erreicht wurden. Die Verfolgung der Radikalkonzentration-Zeit-Verläufe erfolgte bei konstantem Magnetfeld der in Abbildung 10.1 markierten Bande. Zur Verbesserung des Signal-Rausch-Verhältnisses wurden bis zu 50 Scans addiert. In Abbildung 10.4 sind die normierten Radikalkonzentration-ZeitVerläufe nach Eintreffen eines Laserpulses dargestellt.

$\mathrm{Zu}$ beobachten ist, dass die Addition der Initiatorfragmente an das Monomer in der Zeitskala des Experiments instantan geschieht und die Radikalkonzentration mit der Zeit abfällt. Die Zeitachse kann, wie in Abschnitt 3.4.2 beschrieben, in eine Kettenlängenachse der Makroradikale überführt werden, welches Zugriff auf die Kettenlängenabhängigkeit der Terminierung erlaubt. Aufgrund der aus dem konstanten Gewichtsanteil resultierenden abweichenden molaren Konzentrationen und den unterschiedlichen $k_{\mathrm{p}}$ Werten mussten unterschiedliche Zeitskalen für jedes Monomer gewählt werden. 

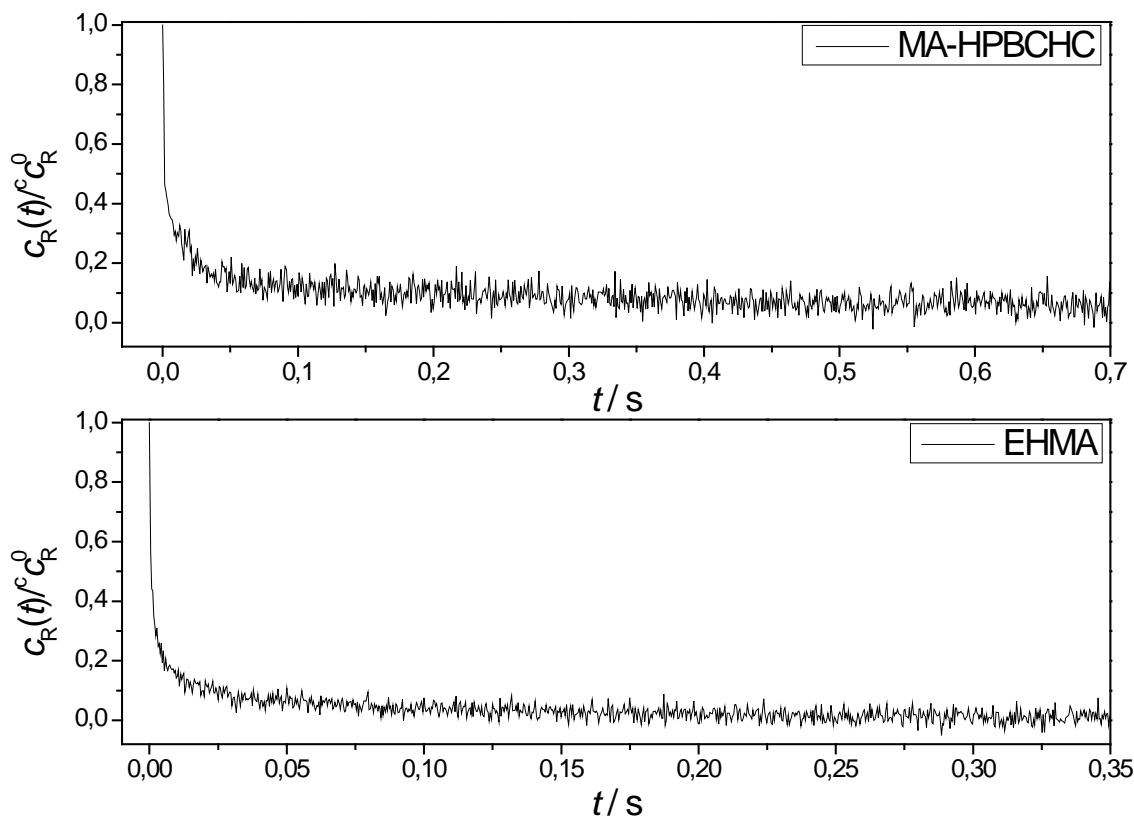

Abbildung 10.4: Normierte Verläufe von ESR Intensität gegen Zeit gemessen für MA-HPBCHC (oben) und EHMA (unten) bei $40^{\circ} \mathrm{C}$. Der Monomeranteil betrug $10 \mathrm{wt} \%$ und die Initiatorkonzentration $50 \mathrm{mmol}$ (Darocure 1173). Die bei Abbildung 10.1 markierte Bande wurde dabei zeitaufgelöst verfolgt. Die Initiierung erfolgte durch einen Laserpuls zum Zeitpunkt $t=0$. Die Zeitauflösung in diesen Messungen wurde zur Analyse des Kurzkettenbereichs ausgewählt.

Zur Bestimmung der composite model Parameter wurde ein Verfahren bestehend aus zwei konsekutiven Schritten verwendet. Zuerst wurde durch doppellogarithmische Auftragung nach Gleichung (3.39) die crossover chain length $i_{\mathrm{c}}$ und der Langkettenbereichexponent $\alpha_{1}$ bestimmt. Nach dem composite model treten zwei Bereiche mit unterschiedlicher Steigung in solch einer Auftragung auf, der Kurz- und der Langkettenbereich. Beide Bereiche wurden durch einen linearen Fit angepasst und die Steigungen ergaben jeweils $1-\alpha_{\mathrm{s}}$ und $1-\alpha_{\mathrm{l}}$. Aus dem Schnittpunkt der beiden Geraden lässt sich $i_{\mathrm{c}}$ nach Gleichung (3.35) bestimmen. Da der Übergang zwischen Kurz- und Langkettenbereich nicht abrupter Natur ist, wurde der Übergangsbereich aus den linearen Anpassungen ausgeschlossen. In Abbildung 10.5 und Abbildung 10.6 sind die doppellogarithmischen 
Auftragungen für jeweils ein Experiment von MA-HPBCHC und EHMA in BL-087 bei $40{ }^{\circ} \mathrm{C}$ dargestellt.

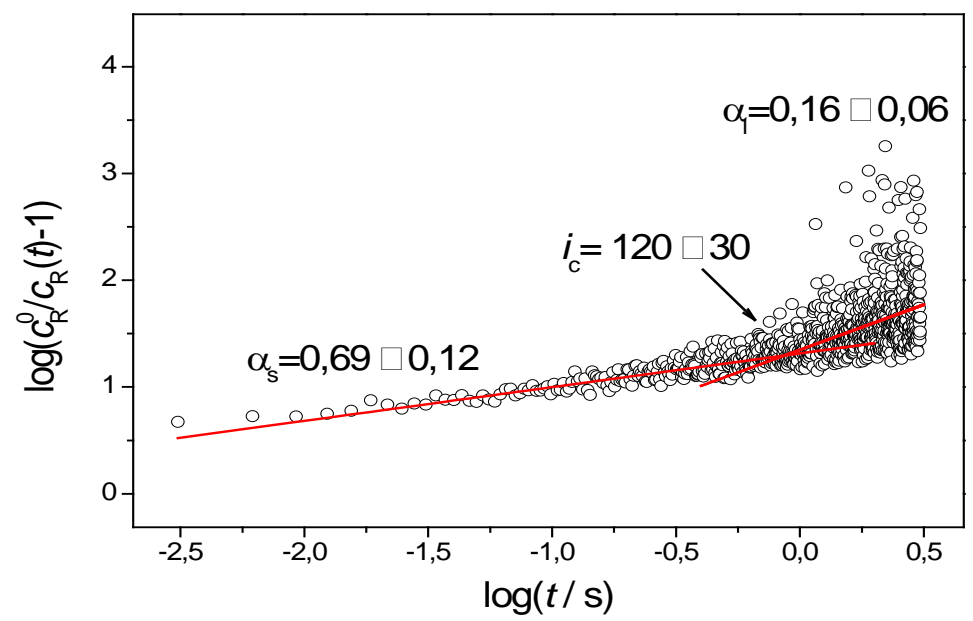

Abbildung 10.5: Doppellogarithmische Auftragung einer SP-PLP-ESR Messung von MA-HPBCHC in BL-087 (10 wt\%) bei $40{ }^{\circ} \mathrm{C}$. Durch Analyse des Langkettenbereiches wurde $\alpha_{1}$ erhalten. $i_{\mathrm{c}}$ wurde aus den Schnittpunkt der linearen Anpassungen von Kurz- und Langkettenbereich erhalten.

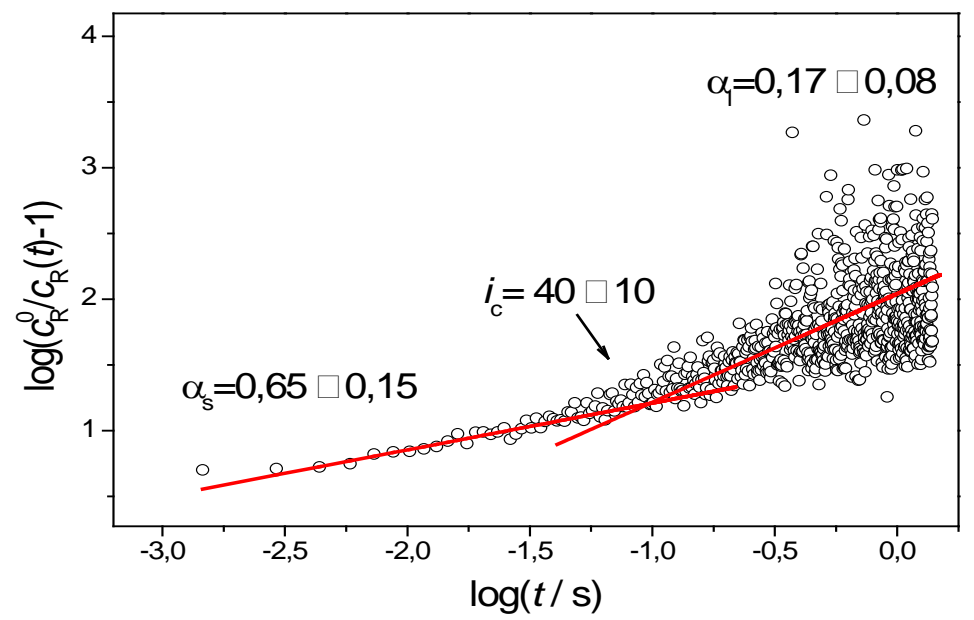

Abbildung 10.6: Doppellogarithmische Auftragung einer SP-PLP-ESR Messung von EHMA in BL-087 (10 wt\%) bei $40{ }^{\circ} \mathrm{C}$. Durch Analyse des Langkettenbereiches wurde $\alpha_{1}$ erhalten. $i_{c}$ wurde aus den Schnittpunkt der linearen Anpassungen von Kurz- und Langkettenbereich erhalten. 
Im Anschluss wurde der Kurzkettenbereich $\left(i<i_{\mathrm{c}}\right)$ untersucht. Dazu wurde $\mathrm{c}_{\mathrm{R}}^{0} / c_{\mathrm{R}}-1$ gegen die Zeit aufgetragen und mittels Gleichung (3.41) mit der Methode der kleinsten Quadrate gefittet. Hierdurch wird $\alpha_{\mathrm{s}}$ und der gekoppelte Parameter $\mathrm{c}_{\mathrm{R}}^{0} \cdot k_{\mathrm{t}}^{1,1}$ erhalten. Aus der in Abschnitt 4.6.3 beschriebenen Kalibrierung kann anschließend $\mathrm{c}_{\mathrm{R}}^{0}$ erhalten und daraus $k_{\mathrm{t}}^{1,1}$ berechnet werden. In Abbildung 10.7 und Abbildung 10.8 sind die bei $40{ }^{\circ} \mathrm{C}$ in BL-087 durchgeführten Kurzkettenbereichanalysen für MA-HPBCHC und EHMA dargestellt. Ein Maß für die Streuung von $\alpha_{\mathrm{s}}$ ist durch die Berechnung von Grenzlinien graphisch dargestellt.

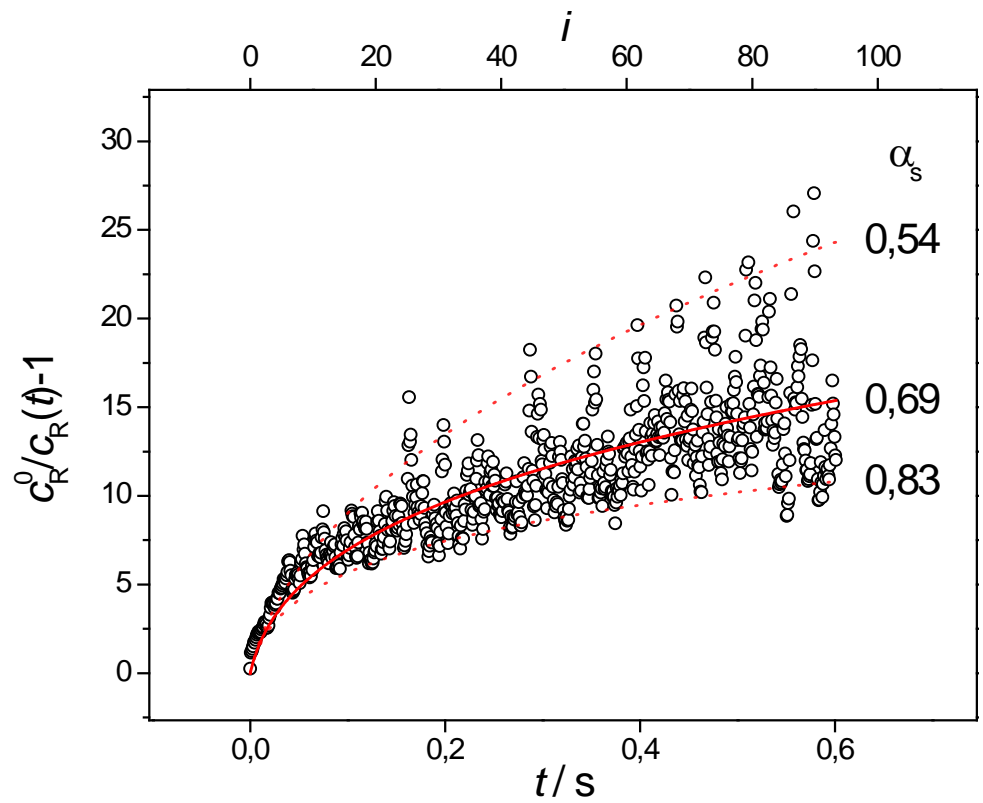

Abbildung 10.7: Fit der SP-PLP-ESR Untersuchung von MA-HPBCHC (10 wt $\%$ in BL-087) durch Auftragung von $c_{R}^{0} / c_{R}-1$ gegen die Zeit $t$ bei $40{ }^{\circ} \mathrm{C}$. Hierdurch wird $\alpha_{\mathrm{s}}$ und der gekoppelte Parameter $c_{\mathrm{R}}^{0} \cdot k_{\mathrm{t}}^{1,1}$ erhalten. Die durchgezogene rote Linie kennzeichnet dabei das beste Ergebnis der Anpassung, während die gestrichelten Linien ein Maß für die Streuung der Messwerte graphisch darstellen. 


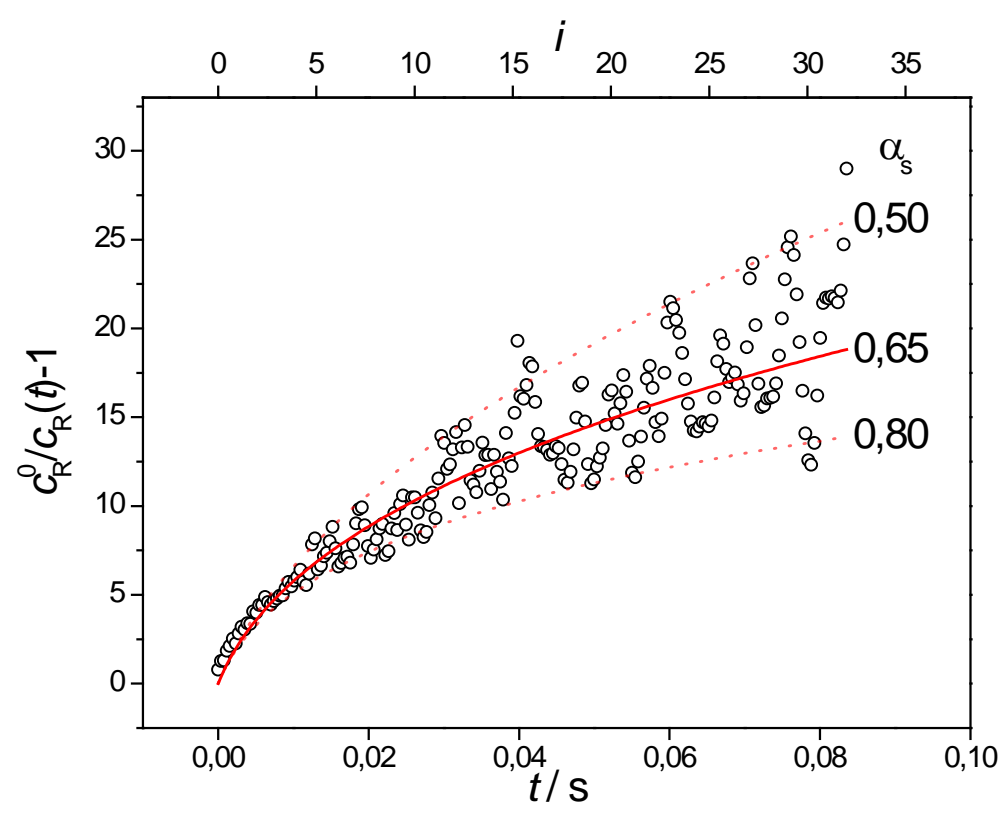

Abbildung 10.8: Fit der SP-PLP-ESR Untersuchung von EHMA (10 wt \% in BL-087) durch Auftragung von $c_{R}^{0} / c_{R}-1$ gegen die Zeit $t$ bei $40{ }^{\circ} \mathrm{C}$. Hierdurch wird $\alpha_{s}$ und der gekoppelte Parameter $c_{R}^{0} \cdot k_{t}^{1,1}$ erhalten. Die durchgezogene rote Linie kennzeichnet dabei das beste Ergebnis der Anpassung, während die gestrichelten Linien ein Maß für die Streuung der Messwerte graphisch darstellen.

\subsubsection{Der Exponent $\alpha_{1}$}

Die Resultate für den Exponenten $\alpha_{1}$ von MA-HPBCHC und EHMA in der nematischen Flüssigkeit BL-087 sind in Abbildung 10.9 zusammengefasst. Signifikante Unterschiede zwischen den Exponenten der zwei Methacrylate sind nicht zu beobachten. Weiterhin ist keine Temperaturabhängigkeit erkennbar. Ein Einfluss der nematischen Anordnung auf den Exponenten $\alpha_{1}$ kann dementsprechend nicht beobachtet werden. Die temperaturgemittelten Werte ergeben $\quad \alpha_{1}$ (EHMA) $=0,17 \pm 0,08$ und $\alpha_{1}(\mathrm{MA}-\mathrm{HPBCHC})=0,18 \pm 0,07$. Diese Ergebnisse stimmen mit den für andere Methacrylate in Substanz berichteten überein. ${ }^{[116,117]}$ Der Literaturwert von EHMA in Substanz $(0,19 \pm 0,05)$ zeigt keine signifikante Abweichung von den hier ermittelten Daten in BL-087. ${ }^{\text {[45] }}$ 


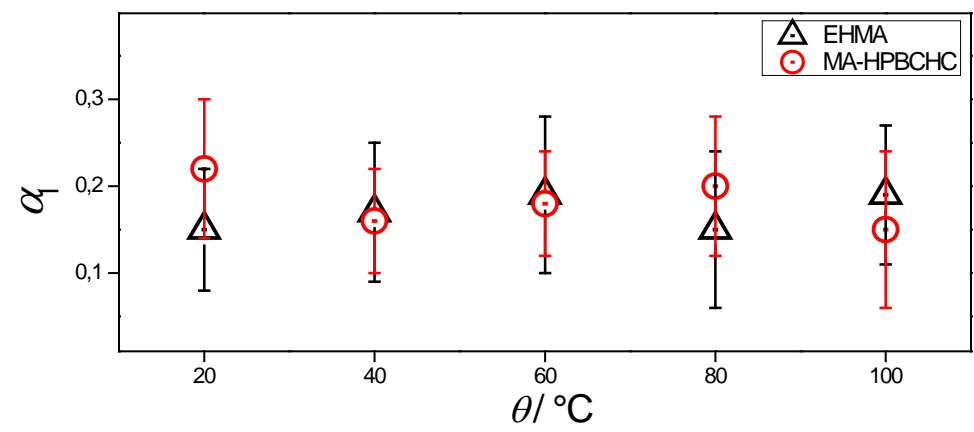

Abbildung 10.9: $\alpha_{1}$ Exponenten von EHMA (schwarze Dreiecke) und MA-HPBCHC (Rote Kreise) gemessen zwischen $20^{\circ} \mathrm{C}$ und $100{ }^{\circ} \mathrm{C}$ in BL-087 (10 wt\%).

Eine Korrelation zwischen Struktur der Seitenkette und $\alpha_{1}$ konnte bis zum jetzigen Zeitpunkt für Methacrylatmonomere nicht beobachtet werden. Weiterhin sind die Abweichungen $\mathrm{zu}$ anderen Monomerfamilien, trotz großer Abweichungen in Struktur und Terminierungskoeffizienten, geringfügig wie im Vergleich mit Butylacrylat $\alpha_{1}(\mathrm{BA})=0,26 \pm 0,07$, Dodecylacrylat $\quad \alpha_{1}(\mathrm{DA})=0,19 \pm 0,07$ und sogar Styrol $\alpha_{1}$ (Styrol) $=0,16 \pm 0,05$ deutlich wird. $^{[118-120]}$ Diese experimentellen Ergebnisse stimmen gut mit dem von der Theorie vorhergesagten Wert von 0,16, welcher für die Terminierung zweier Makroradikale mit der Radikalfunktionalität am Kettenende vorhergesagt wurde. ${ }^{[121]}$

\subsubsection{Der Exponent $\alpha_{\text {s }}$}

Die Ergebnisse für die Exponenten $\alpha_{\mathrm{s}}$ von MA-HPBCHC und EHMA in BL-087 sind in Abbildung 10.10 zusammengefasst. Signifikante Unterschiede zwischen den Exponenten der zwei Methacrylate sind nicht zu beobachten. Eine Temperaturabhängigkeit der Messwerte kann nicht beobachtet werden. Dementsprechend ist ein Einfluss der nematischen Anordnung von BL-087 auf den Exponenten $\alpha_{\mathrm{s}}$ auszuschließen. Die temperaturgemittelten Werte ergeben $\alpha_{\mathrm{s}}$ (EHMA) $=0,61 \pm 0,10$ und $\alpha_{\mathrm{s}}(\mathrm{MA}-\mathrm{HPBCHC})=0,66 \pm 0,11$. Diese Ergebnisse lassen sich gut mit $\alpha_{\mathrm{s}}$ Werte von anderen Methacrylaten in Substanz vergleichen, wie Methylmethacrylat $\left(\alpha_{\mathrm{s}}(\mathrm{MMA})=0,63 \pm 0,15\right)$, Butylmethacrylat $\left(\alpha_{\mathrm{s}}(\mathrm{BMA})=0,65 \pm 0,15\right)$ und Dodecylmethacrylat 
$\left(\alpha_{\mathrm{s}}(\mathrm{DMA})=0,65 \pm 0,08\right){ }^{[45,111,122]}$ Andere Monomerfamilien zeigen Abweichungen in den Exponenten $\alpha_{\mathrm{s}}$. Für Styrol liegt er bei $\alpha_{\mathrm{s}}($ Styrol $)=0,51 \pm 0,05$, während Acrylate Werte um die 0,8 zeigen. ${ }^{[118-120]}$

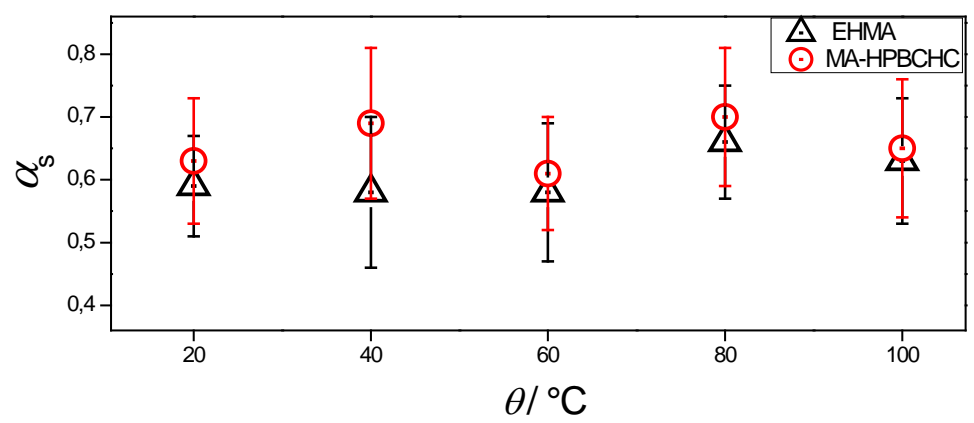

Abbildung 10.10: $\alpha_{\mathrm{s}}$ Exponenten von EHMA (schwarze Dreiecke) und MA-HPBCHC (Rote Kreise) gemessen zwischen $20^{\circ} \mathrm{C}$ und $100{ }^{\circ} \mathrm{C}$ in BL-087 (10 wt\%).

Wie ausführlich in Abschnitt 3.4.2 beschrieben kann der Terminierungsprozess mithilfe der Smoluchowski-Gleichung (3.9) beschrieben werden. Dies erschließt eine Abhängigkeit vom Einfangradius $R_{\mathrm{c}}$ und vom Diffusionskoeffizienten $D^{i}$. Quenchexperimente mit Styrol legen nahe dass $R_{\mathrm{c}}$ unabhängig von der Kettenlänge im Kurzkettenbereich ist, ergo ergibt sich die Kettenlängenabhängigkeit aus $D^{i}{ }^{[123]}$ Dementsprechend ist $\alpha_{\mathrm{s}}$ mit der Verringerung des massenzentrierten Diffussionskoeffizienten mit steigender Kettenlänge verknüpft. Interessanterweise liegen die theoretischen Vorhersagen den experimentellen Ergebnissen recht nahe. Für statistische Knäuel wurde in Lösung ein Koeffizient von 0,5 vorhergesagt. Bei Berücksichtigung des exkludierten Volumens wird $\alpha_{\mathrm{s}}$ auf 0,6 korrigiert. ${ }^{[124]}$

\subsubsection{Die crossover chain length $i_{\mathrm{c}}$}

Die Ergebnisse der Bestimmung der crossover chain length $i_{\mathrm{c}}$ sind in Abbildung 10.11 zusammengefasst. Zu beobachten ist keine systematische Abhängigkeit von $i_{\mathrm{c}}$ von der Temperatur, jedoch eindeutig vom Monomer. Die Mittelwertbildung über alle Temperaturwerte lässt dies deutlich werden, $i_{\mathrm{c}}(\mathrm{EHMA})=35 \pm 10$ und $i_{\mathrm{c}}(\mathrm{MA}-\mathrm{HPBCHC})=100 \pm 25$. 


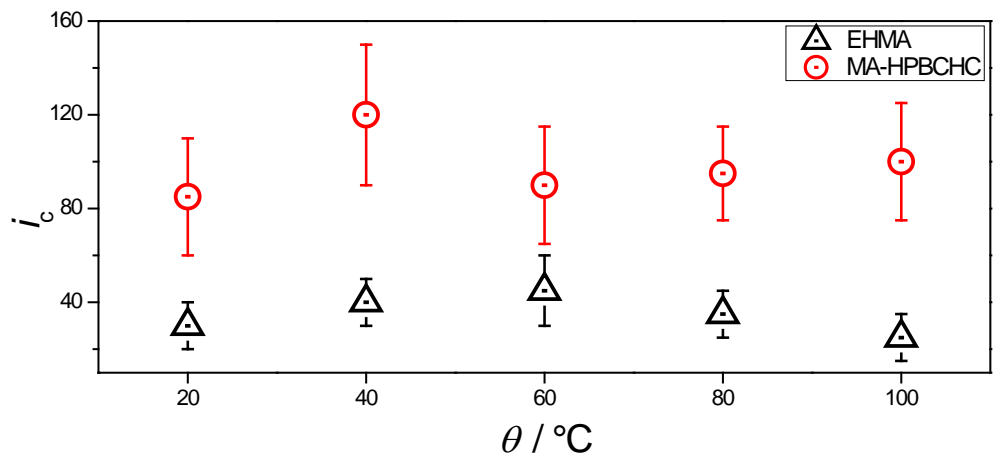

Abbildung 10.11: Crossover chain length $i_{c}$ von EHMA (schwarze Dreiecke) und MA-HPBCHC (Rote Kreise) gemessen zwischen $20^{\circ} \mathrm{C}$ und $100{ }^{\circ} \mathrm{C}$ in BL087 (10 wt \%).

Eine Korrelation zwischen $i_{\mathrm{c}}$ und der Monomerstruktur konnte bis zum heutigen Zeitpunkt nicht festgestellt werden. Im allgemeinen weisen Methacrylate höhere Werte auf als Acrylate, wie am Beispiel Methylmethacrylat $i_{\mathrm{c}}(\mathrm{MMA})=100$ und Methylacrylat $i_{\mathrm{c}}(\mathrm{MA})=35 \pm 10$ deutlich wird. ${ }^{[120,120,125]}$ Der Parameter $i_{\mathrm{c}}$ wird gewöhnlicherweise mit der Mobilität der Makroradikalketten in Verbindung gebracht. Große Substituenten sollen diese einschränken, was zu einer Verschiebung des Überganges von Translationsdiffusionskontrolle zu Segmentsdifussionskontrolle zu höheren Kettenlängen führt. Dafür spricht das schon angesprochene Verhältnis von Methacrylaten zu Acrylaten und die bei Acrylaten steigenden $i_{\mathrm{c}}$-Werte mit größer werdender Seitenkette. ${ }^{[120]}$ Weiterhin interessant ist die von Sörensen beobachtete Temperaturabhängigkeit für EHMA und DMA. ${ }^{[45]}$ Für EHMA stellte er $i_{\mathrm{c}^{-}}$ Werte von $285 \pm 40$ bei $233 \mathrm{~K}$ bis $80 \pm 15$ bei $333 \mathrm{~K}$ fest. Diese Ergebnisse unterstützen die oben aufgestellte Vermutung indem die temperaturinduzierte erhöhte Kettenmobilität als Erklärung verwendet wird. Weiterhin scheint $i_{\mathrm{c}}$ abhängig von der Wahl des Lösungsmittels zu sein, wie Messungen verschiedener Acrylaten in Toluol und von ((Poly(Ethylenglycol))methylether)methylmethacrylat in Wasser ergaben. ${ }^{[120,126]}$ Aus diesen Gründen Reihen sich die Resultate der hier vorgestellten Messungen gut in vorrangegangene Ergebnisse ein. Die Struktur der Makroradikale wird nicht durch die nematische Phase beeinflusst, wie die Temperaturabhängigkeit der Messergebnisse zeigt. $\mathrm{Zu}$ 
erwarten in diesem Fall wäre bei einer Ausrichtung der Makroradikale in der Flüssigkristallinen Phase eine Abnahme von $i_{\mathrm{c}}$ mit der Temperatur. MA-HPBCHC zeigt durch seine sterisch deutlich anspruchsvollere Seitengruppe deutlich höhere $i_{\mathrm{c}}$ Werte als EHMA. Die Abweichung der $i_{\mathrm{c}^{-}}$ Werte für EHMA in Bulk und BL-087 ergeben sich vermutlich aus einer unterschiedlichen Kettenmobilität aufgrund der variierenden Wechselwirkungen mit dem Lösungsmittel.

\subsubsection{Der Terminierungskoeffizient $\boldsymbol{k}_{\mathrm{t}}^{1,1}$}

Der Terminierungskoeffizient $k_{\mathrm{t}}^{1,1}$ wurde durch Fitten von Gleichung (4.6) mittels der Methode der kleinsten Quadrate aus dem gekoppelten Parameter $k_{\mathrm{t}}^{1,1} \cdot c_{\mathrm{R}}^{0}$ bestimmt. Die Radikalkonzentration wurde aus der Signalintensität bei Laserpulsauftritt mittels TEMPO Kalibrierung (siehe Abschnitt 4.6.3) erhalten. Durch eine Auftragung nach Arrhenius wurde die Temperaturabhängigkeit des Terminierungskoeffizienten analysiert.

Der Terminierungskoeffizient von EHMA in der nematischen Flüssigkeit BL-087 (10 wt\%) wurde in einem Temperaturbereich von $20^{\circ} \mathrm{C}$ bis $100^{\circ} \mathrm{C}$ in $20^{\circ} \mathrm{C}$ Schritten untersucht. Der Klärpunkt der Lösung lag hierbei bei $58^{\circ} \mathrm{C}$, somit lagen die Messwerte die bei $20^{\circ} \mathrm{C}$ und $40^{\circ} \mathrm{C}$ aufgenommen wurden in der nematischen Phase der Mischung. In Abbildung 10.12 ist der Arrhenius-Plot für die Terminierungskoeffizienten von EHMA in der nematischen Phase von BL-087 (10 wt\%) dargestellt. Die Werte zeigen keine Abweichung von der Linearität, unabhängig davon ob sie in der nematischen oder in der isotropen Phase aufgenommen wurden. Dementsprechend kann, wie schon zuvor für die anderen composite model Parameter, kein anisotroper Effekt beobachtet werden. Die Temperaturabhängigkeit des Terminierungskoeffizienten $k_{\mathrm{t}}^{1,1}$ von EHMA in BL-087 lässt sich durch folgenden Zusammenhang beschreiben:

$$
\ln \left(k_{\mathrm{t}}^{1,1} / \mathrm{L} \mathrm{mol}^{-1} \mathrm{~s}^{-1}\right)=22,04-1670 /(T / \mathrm{K})
$$




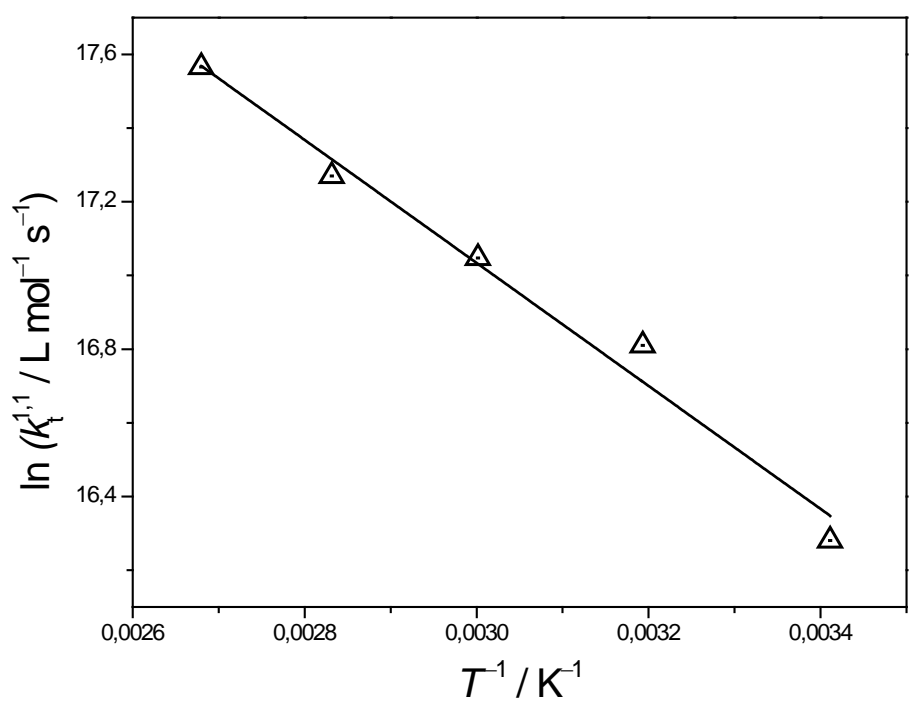

Abbildung 10.12: Arrhenius-Plot der Terminierungskoeffizienten $k_{t}^{1,1}$ von EHMA in BL-087 (10 wt\%) erhalten aus SP-PLP-ESR Messungen.

Für MA-HPBCHC wurde der gleiche Temperaturbereich in der nematischen Flüssigkeit BL-087 (10 wt\%) untersucht wie für EHMA. Der Klärpunkt der Lösung lag in dieser Mischung bei $76^{\circ} \mathrm{C}$. Dementsprechend gehören die Messwerte bei $20^{\circ} \mathrm{C}, 40^{\circ} \mathrm{C}$ und $60^{\circ} \mathrm{C}$ zu der nematischen Phase der Mischung. Die Arrhenius Auftragung von $k_{\mathrm{t}}^{1,1}$ für MA-HPBCHC ist in Abbildung 10.13 dargestellt. Die Werte in der nematischen und isotropen Phase zeigen keine substantielle Abweichung von der Linearität. Wie schon zuvor für EHMA und den restlichen composite model Parameter von MA-HPBCHC, kann kein anisotroper Effekt beobachtet werden. Die Temperaturabhängigkeit des Terminierungskoeffizienten $k_{\mathrm{t}}^{1,1}$ von MA-HPBCHC in BL-087 lässt sich durch folgenden Zusammenhang beschreiben:

$$
\ln \left(k_{\mathrm{t}}^{1,1} / \mathrm{L} \mathrm{mol}^{-1} \mathrm{~s}^{-1}\right)=20,87-1417 /(T / \mathrm{K})
$$




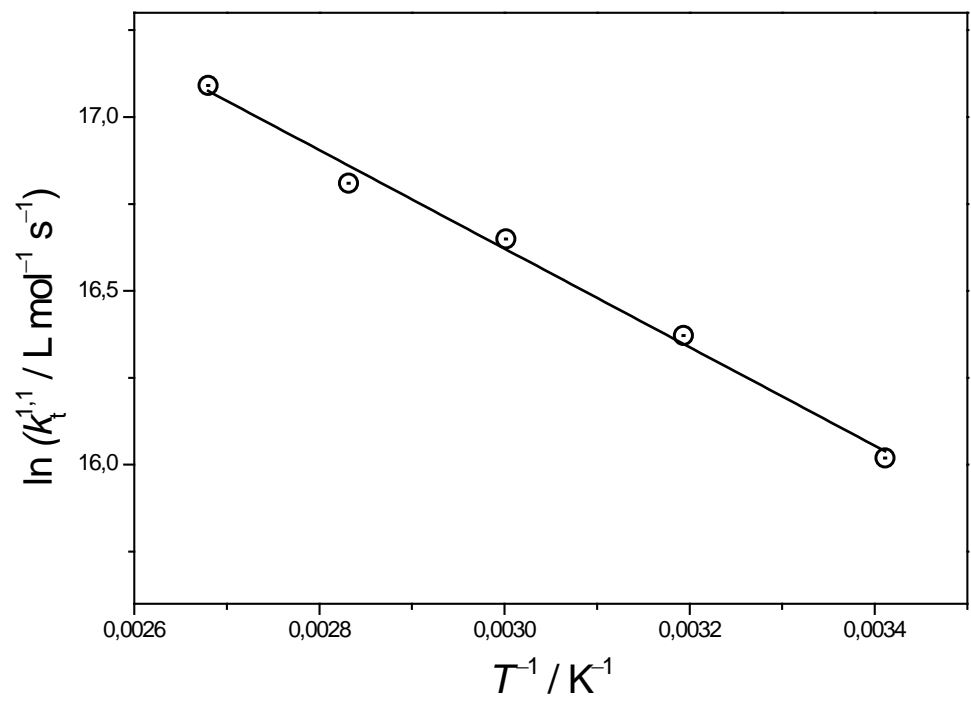

Abbildung 10.13: Arrhenius-Plot der Terminierungskoeffizienten $k_{t}^{1,1}$ von MA-HPBCHC in BL-087 (10 wt\%) erhalten aus SP-PLP-ESR Messungen.

Aus (10.2) und (10.3) ergeben sich die Aktivierungsenergien der Terminierungskoeffizienten $\mathrm{zu} \quad E_{\mathrm{A}}(\mathrm{EHMA})=13,9 \pm 1,0 \mathrm{~kJ} \mathrm{~mol}^{-1}$ und $E_{\mathrm{A}}(\mathrm{MA}-\mathrm{HPBCHC})=11,8 \pm 0,6 \mathrm{~kJ} \mathrm{~mol}^{-1}$. Wie ausführlich in Abschnitt 3.4.2 diskutiert ist die Terminierung ein diffusionskontrollierter Prozess und somit sollte die Aktivierungsenergie von $k_{\mathrm{t}}^{1,1}$ proportional $\mathrm{zu}$ derjenigen der inversen Viskosität $\eta^{-1}$ sein. Dies gilt unter der Annahme einer isotropen Lösung. Aufgrund von fehlenden Anisotropieeffekten in den Messungen scheint die Annahme naheliegend, dass der Mechanismus der Terminierung in nematischen Flüssigkeiten dem in isotroper Lösung gleicht. Im Falle von Flüssigkristallen ist die Messung einer Viskosität welches mit der Diffusionsgeschwindigkeit in Verbindung gesetzt werden kann jedoch nicht trivial. ${ }^{[127-129]}$ Dementsprechend bleibt ein Vergleich von $k_{\mathrm{t}}^{1,1}$ mit der Fluidität der Lösung aus.

In nematischen Flüssigkristallen ist die Anisotropie des Diffusionskoeffizienten eine literaturbekannte Tatsache. ${ }^{[130-133]}$ Dies bedeutet, dass Translationsdiffusion abhängig von der Diffusionsrichtung in Bezug zum Direktor der Phase ist. Die hier verwendete Methode hat keine räumliche Auflösung und bietet dementsprechend keine Informationen über 
richtungsabhängige Diffusion. Die ermittelten Koeffizienten sind als Mittelwert derjenigen in alle Raumrichtungen zu betrachten. Dass sich die Messwerte vollständig mit dem composite model beschreiben lassen, die Parameter $\alpha_{\mathrm{s}}, \alpha_{\mathrm{l}}$ und $i_{\mathrm{c}}$ temperaturunabhängig sind und die Arrhenius Auftragung von $k_{\mathrm{t}}^{1,1}$ Linearität aufweist sind starke Hinweise auf fehlende Anisotropie der Diffusionsprozesse von Makroradikalen in nematischen Flüssigkeiten. Ein Grund dafür könnte eine lokale Störung der Ordnung durch die Makromoleküle sein.

Die erhaltenen Messwerte können mit Literaturwerte aus isotroper Phase ins Verhältnis gestellt werden. Der Vergleich mit Literaturwerten vom Terminierungskoeffizienten in Substanz von anderen Methacrylaten (siehe Tabelle 10.1) zeigt, dass sich die Werte in einer ähnlichen Größenordnung befinden. Der Vergleich der Terminierungsraten von EHMA in Substanz und in BL-087 zeigt eine nachvollziehbare Verringerung von $k_{\mathrm{t}}^{1,1}$, die durch die optisch erkennbare höhere Viskosität der flüssigkristallinen Lösungen herrührt.

Tabelle 10.1: Übersicht über die Terminierungskoeffizienten $k_{t}^{1,1}$ bei $20^{\circ} \mathrm{C}$ verschiedener Methacrylate in Substanz und in der nematischen Flüssigkeit BL-087.

\begin{tabular}{ccc}
\hline Monomer & Lösungsmittel & $k_{\mathrm{t}}^{1,1}\left(20^{\circ} \mathrm{C}\right) \cdot 10^{-7} /$ \\
& $\mathrm{L} \mathrm{mol}^{-1} \mathrm{~s}^{-1}$ \\
\hline MMA $^{[134]}$ & - & $60 \pm 20$ \\
EHMA $^{[45]}$ & - & $3,0 \pm 0,5$ \\
DMA $^{[45]}$ & - & $2,3 \pm 0,4$ \\
EHMA $_{\text {MA-HPBCHC }}$ & BL-087 & $1,3 \pm 0,5$ \\
& BL-087 & $0,9 \pm 0,4$ \\
\hline
\end{tabular}




\subsection{Terminierung von Methacrylaten in chiral-nematischer Phase}

Nachdem die Terminierung von EHMA und MA-HPBCHC in nematischer Phase erläutert wurde soll nun der Einfluss von der Zugabe eines chiralen Dotierstoffes vorgestellt werden. Durch Zugabe von 5 und 7 wt\% R-5011 wurden chiral-nematische Phasen mit unterschiedlicher helikalen Ganghöhe erzeugt. Der Klärpunkt der Mischungen veränderte sich durch Zugabe von R-5011. Die Mischungen mit EHMA zeigten den Übergang in die isotrope Phase bei $47^{\circ} \mathrm{C}$ (5 wt\% R-5011) und $42{ }^{\circ} \mathrm{C}$ (7 wt\% $\mathrm{R}-5011$ ). Die Mischungen mit MA-HPBCHC wiesen einen Klärpunkt bei $70{ }^{\circ} \mathrm{C}$ (5 wt\% $\mathrm{R}-5011)$ und $62{ }^{\circ} \mathrm{C}(7 \mathrm{wt} \% \mathrm{R}-5011)$ auf. Die Terminierung von EHMA und MA-HPBCHC (10 wt\%) wurde in diesen Mischungen, wie ausführlich in Abschnitt 10.3 erklärt, zwischen $20^{\circ} \mathrm{C}$ und $100{ }^{\circ} \mathrm{C}$ untersucht. Die Ergebnisse der composite model Parameter $\alpha_{\mathrm{s}}, \alpha_{\mathrm{l}}$ und $i_{\mathrm{c}}$ sind für EHMA in Abbildung 10.14 und für MA-HPBCHC in Abbildung 10.15 dargestellt.

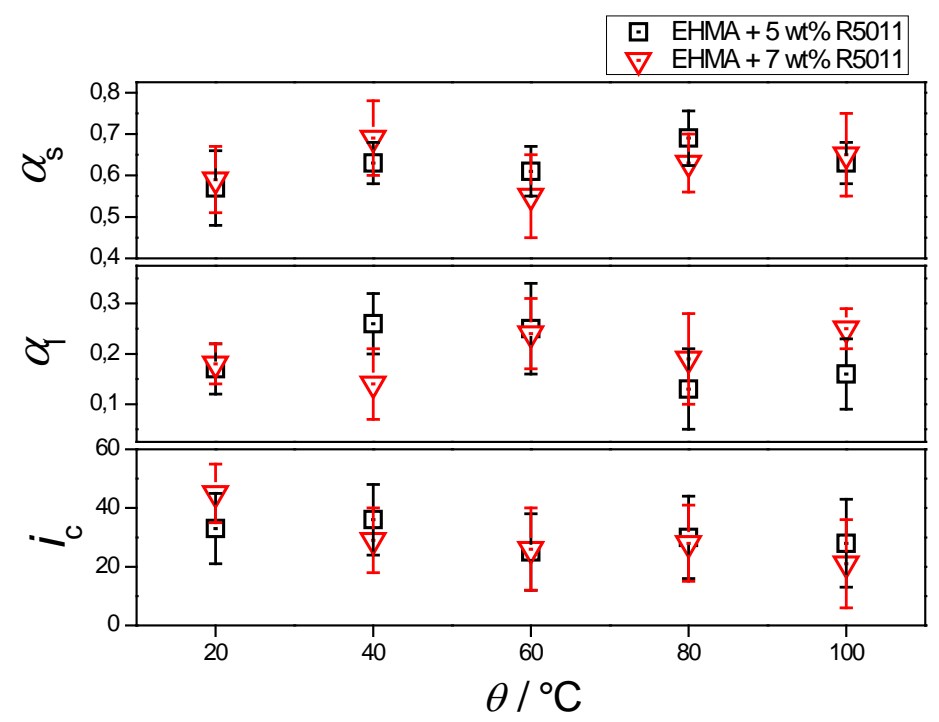

Abbildung 10.14: $\alpha_{\mathrm{s}}, \alpha_{1}$ und $\boldsymbol{i}_{\mathrm{c}}$ von EHMA in chiral-nematischer Phase. Die schwarzen Vierecke kennzeichnen die Messwerte in BL-087 mit 5 wt\% R-5011 und die roten Dreiecke die Messwerte in BL-087 mit 7 wt\% R-5011. 


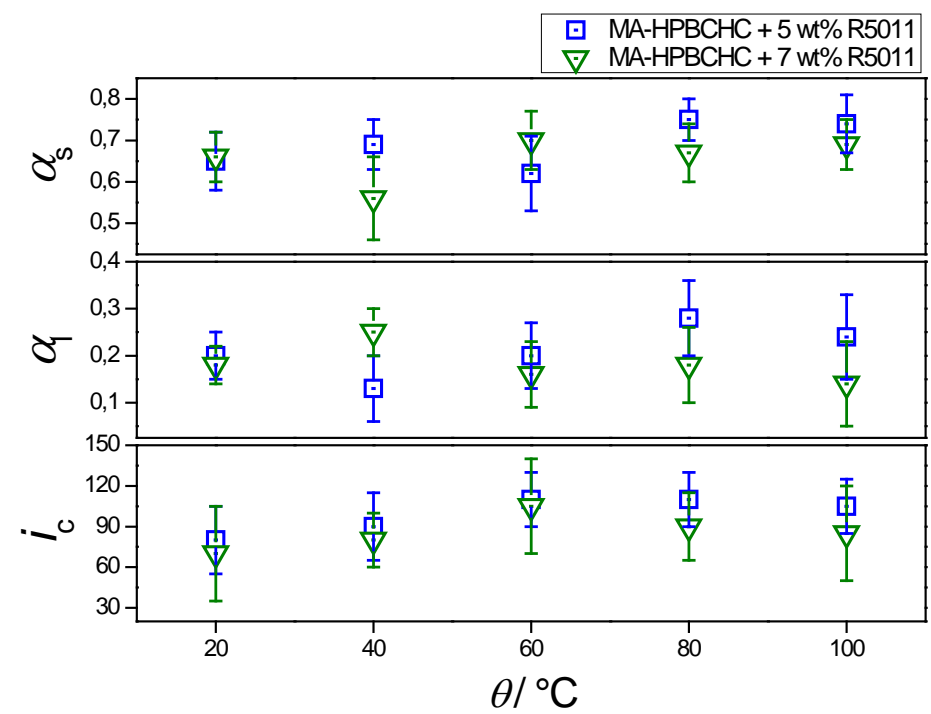

Abbildung 10.15: $\alpha_{\mathrm{s}}, \alpha_{1}$ und $i_{\mathrm{c}}$ von MA-HPBCHC in chiral-nematischer Phase. Die blauen Vierecke kennzeichnen die Messwerte in BL-087 mit 5 wt\% R-5011 und die grünen Dreiecke die Messwerte in BL-087 mit 7 wt\% R-5011.

$\mathrm{Zu}$ beobachten ist, wie schon für die nematische Lösungen in Abschnitt 10.3 beschrieben, keine signifikanten Abweichungen der Parameter $\alpha_{\mathrm{s}}, \alpha_{1}$ und $i_{\mathrm{c}}$ mit Temperaturveränderung. Zum Vergleich mit den erhaltenen Werten in der nematischen Flüssigkeit sind die temperaturgemittelten Werte dieser Parameter in Tabelle 10.2 aufgelistet.

Die Messwerte zeigen keinen signifikanten Einfluss durch Zugabe von R5011 auf die Parameter $\alpha_{\mathrm{s}}, \alpha_{1}$ und $i_{\mathrm{c}}$ von EHMA und MA-HPBCHC. Dies deutet daraufhin, dass das Lösungsverhalten der Makroradikale durch Zugabe des chiralen Dotierstoffes unverändert bleibt. Diese Tatsache ergänzt die Ergebnisse von Abschnitt 10.3 in dem Sinne eines fehlenden Anisotropieeffekts, welcher auch nicht in chiral-nematischer Phase beobachtet werden konnte. Dies scheint darauf hinzuweisen, dass die Anisotropie der Lösung keinen Einfluss auf das Lösungsverhalten der Polymerknäuel von EHMA und MA-HPBCHC aufzuweisen scheint. 
Tabelle 10.2: Übersicht der temperaturgemittelten Parameter $\alpha_{\mathrm{s}}, \alpha_{1}$ und $\boldsymbol{i}_{\mathrm{c}}$ von EHMA und MA-HPBCHC in BL-087 und BL-087 dotiert mit dem chiralen Dotierstoff R-5011.

\begin{tabular}{|c|c|c|c|c|}
\hline Monomer & Lösungsmittel & $\alpha_{\mathrm{s}}$ & $\alpha_{1}$ & $i_{\mathrm{c}}$ \\
\hline EHMA & BL-087 & $0,61 \pm 0,10$ & $0,17 \pm 0,08$ & $35 \pm 10$ \\
\hline EHMA & $\begin{array}{c}\text { BL-087 + } 5 w t \% \\
\text { R-5011 }\end{array}$ & $0,63 \pm 0,06$ & $0,19 \pm 0,06$ & $30 \pm 13$ \\
\hline EHMA & $\begin{array}{c}\text { BL-087 + } 7 \text { wt } \% \\
\text { R-5011 }\end{array}$ & $0,63 \pm 0,09$ & $0,20 \pm 0,06$ & $30 \pm 12$ \\
\hline $\begin{array}{c}\text { MA- } \\
\text { HPBCHC }\end{array}$ & BL-087 & $0,66 \pm 0,11$ & $0,18 \pm 0,07$ & $100 \pm 25$ \\
\hline $\begin{array}{c}\text { MA- } \\
\text { HPBCHC }\end{array}$ & $\begin{array}{c}\text { BL-087 + } 5 w t \% \\
\text { R-5011 }\end{array}$ & $0,69 \pm 0,08$ & $0,21 \pm 0,07$ & $100 \pm 20$ \\
\hline $\begin{array}{c}\text { MA- } \\
\text { HPBCHC }\end{array}$ & $\begin{array}{c}\text { BL-087 + } 7 \text { wt } \% \\
\text { R-5011 }\end{array}$ & $0,66 \pm 0,08$ & $0,18 \pm 0,07$ & $90 \pm 30$ \\
\hline
\end{tabular}

Im Gegensatz zu den zuvor vorgestellten Ergebnissen, kann ein klarer Effekt durch die Zugabe von R-5011 auf $k_{\mathrm{t}}^{1,1}$ beobachtet werden. Der Arrhenius Auftragung der erhaltenen Messwerte für EHMA und MA-HPBCHC bei Zugabe von 5 und 7 wt\% R-5011 sind zusätzlich zu den in Abschnitt 10.3 bestimmten Werten in den Abbildung 10.16 und Abbildung 10.17 dargestellt.

$\mathrm{D}$

Abbildung 10.16: Arrhenius-Plot der Terminierungskoeffizienten $k_{t}^{1,1}$ von EHMA (10 wt\%) in BL-087 (schwarze Vierecke), BL-087 mit 5 wt\% R-5011 (rote Kreise) und BL-087 mit 7 wt\% R-5011 (blaue Dreiecke) erhalten aus SP-PLP-ESR Messungen. Die durchgezogenen Linien kennzeichnen den jeweiligen linearen Fit. 
Aus der Arrhenius Auftragung ergibt sich die Temperaturabhängigkeit von $k_{\mathrm{t}}^{1,1}$ (EHMA) in BL-087 mit 5 und 7 wt\% R-5011 zu den Gleichungen (10.4) und (10.5).

$$
\begin{aligned}
& \ln \left(k_{\mathrm{t}}^{1,1} / \mathrm{L} \mathrm{mol}^{-1} \mathrm{~s}^{-1}\right)=24,33-2283 /(T / \mathrm{K}) \\
& \ln \left(k_{\mathrm{t}}^{1,1} / \mathrm{L} \mathrm{mol}^{-1} \mathrm{~s}^{-1}\right)=27,00-3322 /(T / \mathrm{K})
\end{aligned}
$$

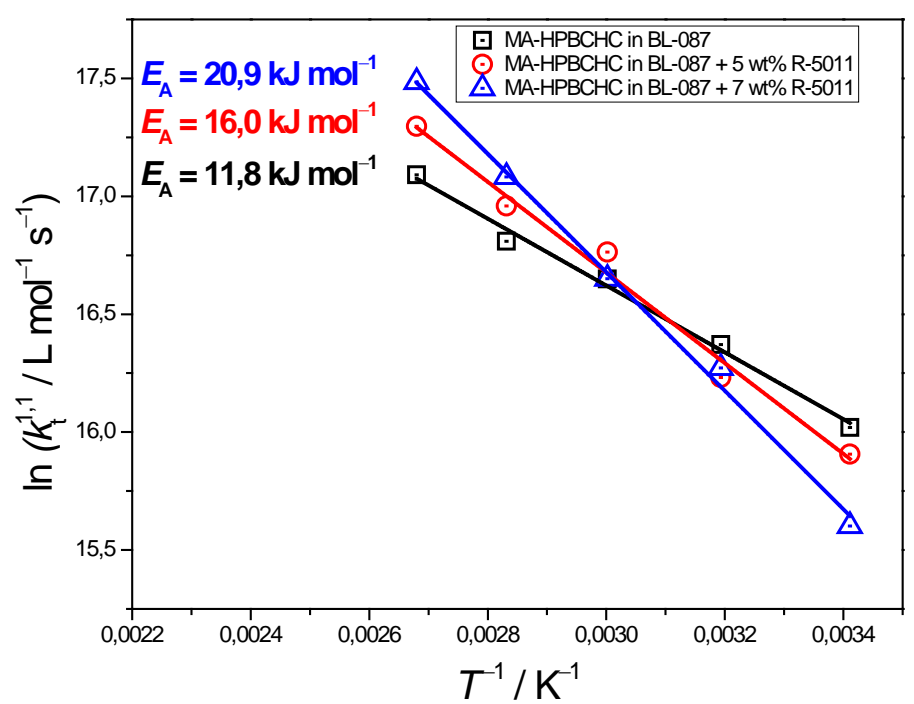

Abbildung 10.17: Arrhenius-Plot der Terminierungskoeffizienten $k_{\mathrm{t}}^{1,1}$ von MA-HPBCHC (10 wt \%) in BL-087 (schwarze Vierecke), BL-087 mit 5 wt \% R-5011 (rote Kreise) und BL-087 mit 7 wt\% R-5011 (blaue Dreiecke) erhalten aus SP-PLP-ESR Messungen. Die durchgezogenen Linien kennzeichnen den jeweiligen linearen Fit.

Für MA-HPBCHC in BL-087 mit 5 und 7 wt\% R-5011 lässt sich die Temperaturabhängigkeit von $k_{\mathrm{t}}^{1,1}$ aus dem Arrhenius-Plot mithilfe der Gleichungen (10.6) und (10.7) darstellen. 


$$
\begin{aligned}
& \ln \left(k_{\mathrm{t}}^{1,1} / \mathrm{L} \mathrm{mol}^{-1} \mathrm{~s}^{-1}\right)=22,44-1922 /(T / \mathrm{K}) \\
& \ln \left(k_{\mathrm{t}}^{1,1} / \mathrm{L} \mathrm{mol}^{-1} \mathrm{~s}^{-1}\right)=24,21-2512 /(T / \mathrm{K})
\end{aligned}
$$

$\mathrm{Zu}$ beobachten ist eine Zunahme der Aktivierungsenergie von $k_{\mathrm{t}}^{1,1}$ mit zunehmenden Anteil von R-5011 für beide Monomere. Im Falle von EHMA bedeutet dies $E_{\mathrm{A}}=13,9 \pm 1 \mathrm{~kJ} \mathrm{~mol}^{-1}$ (in BL-087), $E_{\mathrm{A}}=19,0 \pm 0,6 \mathrm{~kJ} \mathrm{~mol}^{-1} \quad$ (in BL-087 mit $5 \mathrm{wt} \%$ R-5011) und $E_{\mathrm{A}}=27,6 \pm 1 \mathrm{~kJ} \mathrm{~mol}^{-1}$ (in BL-087 mit $7 \mathrm{wt} \% \mathrm{R}-5011$ ). Im Falle von MA-HPBCHC bedeutet dies $E_{\mathrm{A}}=11,8 \pm 0,6 \mathrm{~kJ} \mathrm{~mol}^{-1}$ (in BL-087), $E_{\mathrm{A}}=16,0 \pm 1 \mathrm{~kJ} \mathrm{~mol}^{-1} \quad$ (in BL-087 mit $5 \mathrm{wt} \%$ R-5011) und $E_{\mathrm{A}}=20,9 \pm 0,8 \mathrm{~kJ} \mathrm{~mol}^{-1}$ (in BL-087 mit $7 \mathrm{wt} \% \mathrm{R}-5011$ ). Dies spricht für eine Beeinflussung der Mikroviskosität der Lösung durch die Zugabe von R-5011, welche die Diffusionsgeschwindigkeit in der Lösung beeinflusst. Dieser Effekt kann nicht durch die Anisotropie der Lösung erklärt werden, denn, wie zuvor in Abschnitt 10.3, die Werte in isotroper Phase und anisotroper Phase einen linearen Zusammenhang im Arrheniusplot zeigen.

In diesem Kapitel wurde die Terminierung in nematischer und chiralnematischer Phase von EHMA und MA-HPBCHC vorgestellt. Es konnte die Kettenlängenabhängigkeit der Terminierung in den untersuchten flüssigkristallinen Lösungen nachgewiesen werden. Dabei zeigten sich die Parameter des composite model $\alpha_{\mathrm{s}}, \alpha_{1}$ und $i_{\mathrm{c}}$ unabhängig von der Temperatur und nahe den Literaturwerten für andere Methacrylaten in Substanz. Der Terminierungskoeffizient $k_{\mathrm{t}}^{1,1}$ von EHMA in nematischer Phase wich von dem Wert in Substanz ab und wurde durch Zugabe eines chiralen Dotierstoffes weiterhin maßgeblich beeinflusst. Die $k_{\mathrm{t}}^{1,1}$ Werte von MA-HPBCHC, welche in BL-087 gemessen wurden, wurden ebenfalls durch Zugabe eines chiralen Dotierstoffes beeinflusst. Diese Ergebnisse führen zu der Schlussfolgerung, dass der Terminierungsmechanismus der untersuchten Monomere in dem hier verwendeten flüssigkristallinen Lösungsmittel dem in isotroper Phase gleicht. Der Einfluss auf $k_{\mathrm{t}}^{1,1}$ ist ein 
Effekt der Diffusionsgeschwindigkeit die aus der Fluidität der Lösung entstammt und bei jeder Mischung durch Wechselwirkung der einzelnen Komponenten variiert. 


\section{Ausblick}

Diese Arbeit eröffnet ein weites Feld an weiteren Forschungsvorhaben zur Kinetik von radikalischen Polymerisationen in Flüssigkristallen. Die vorgestellten Untersuchungen fokussierten sich auf grundlegende Reaktionsschritte und fanden dementsprechend bei niedrigen Umsätzen statt. Die Untersuchung der Umsatzabhängigkeit der radikalischen Polymerisation in Flüssigkristallen ist weiterhin von größtem Interesse.

Phasenseparation ist ein weit verbreitetes Phänomen bei der Polymerisation in Flüssigkristallen. ${ }^{[135,136]}$ Die meisten Polymere lösen sich nur sehr eingeschränkt in flüssigkristallinen Lösungsmittel. Somit kommt es zur Bildung von Phasentrennung, insbesondere bei hohen Umsätzen und mittleren Molmassen. Die daraus entstehenden Konzentrationsgradienten und Änderungen in der Viskosität erhöhen im relevanten Maße die Komplexität der Systeme. Zur Beschreibung des gesamten Reaktionsverlaufes durch ein kinetisches Modell müssen tiefergehende Untersuchungen in dieses Gebiet erfolgen.

Auch der Effekt von anderen flüssigkristallinen Phasen, wie die smektischen Phasen oder die blue phase, auf das Terminierungsverhalten von Makroradikalen ist noch nicht untersucht worden. 


\section{Anhang}

\subsection{Dichtemessungen}

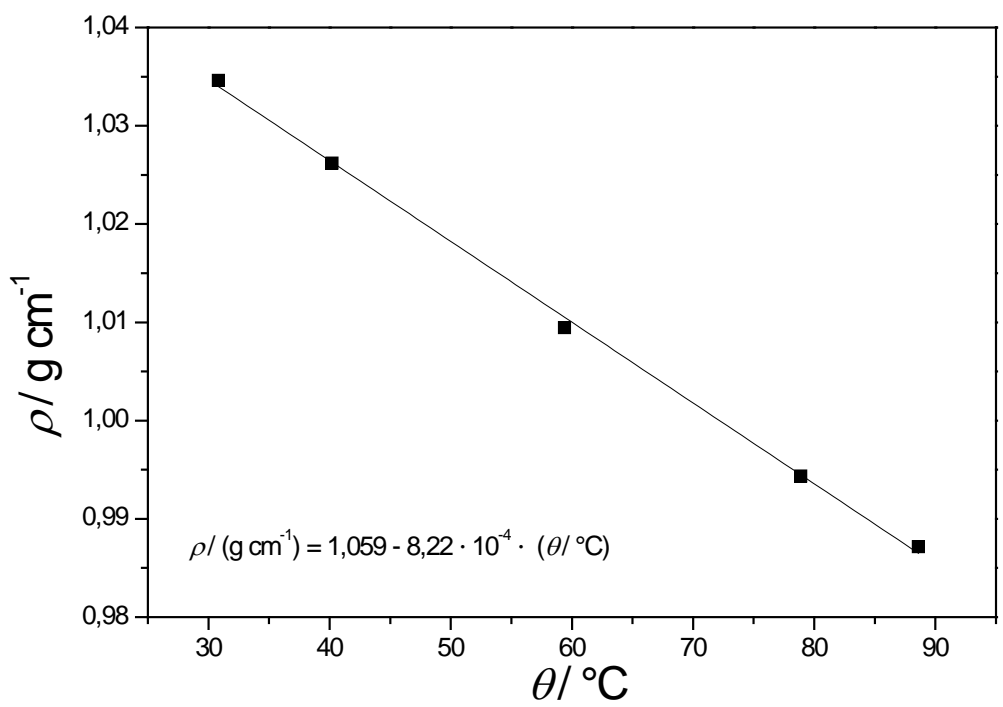

Abbildung 12.1: Dichte als Funktion der Temperatur für EHMA (10 wt\%) in BL-087. 


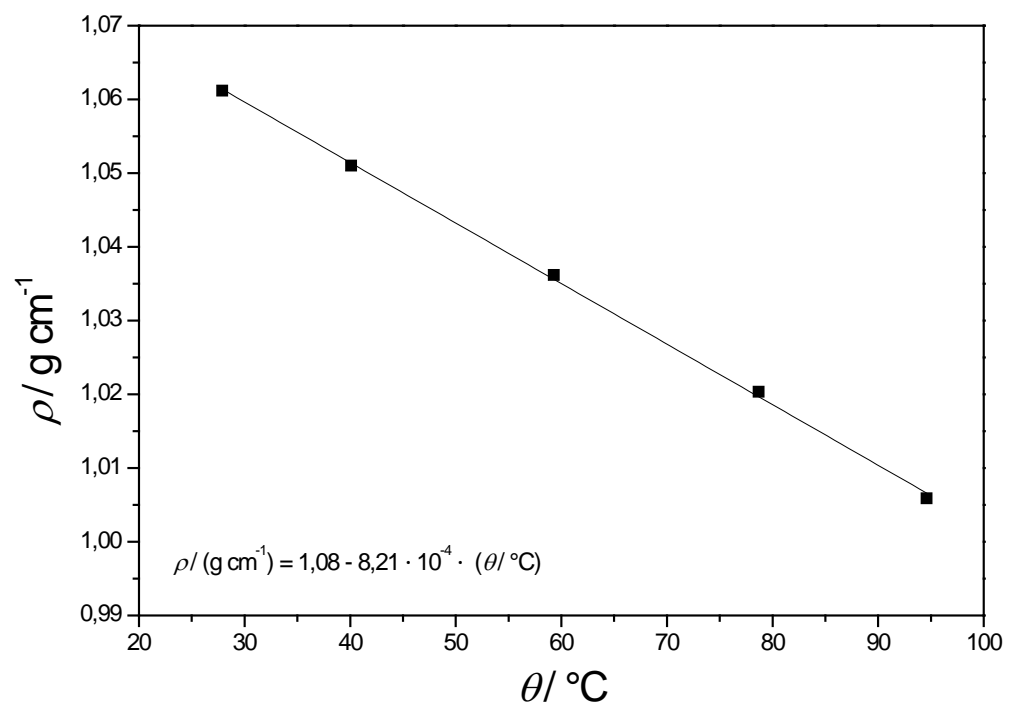

Abbildung 12.2: Dichte als Funktion der Temperatur für EHMA (10 wt\%) in BL-087 mit R-5011 (5 wt\%).

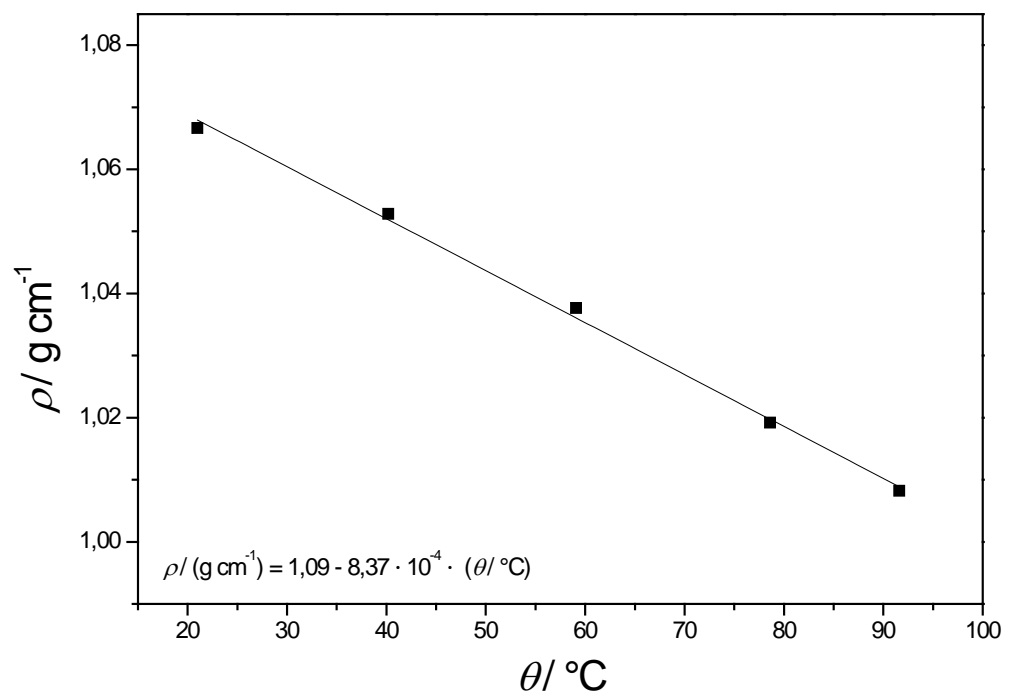

Abbildung 12.3: Dichte als Funktion der Temperatur für EHMA (10 wt\%) in BL-087 mit R-5011 (7 wt\%). 


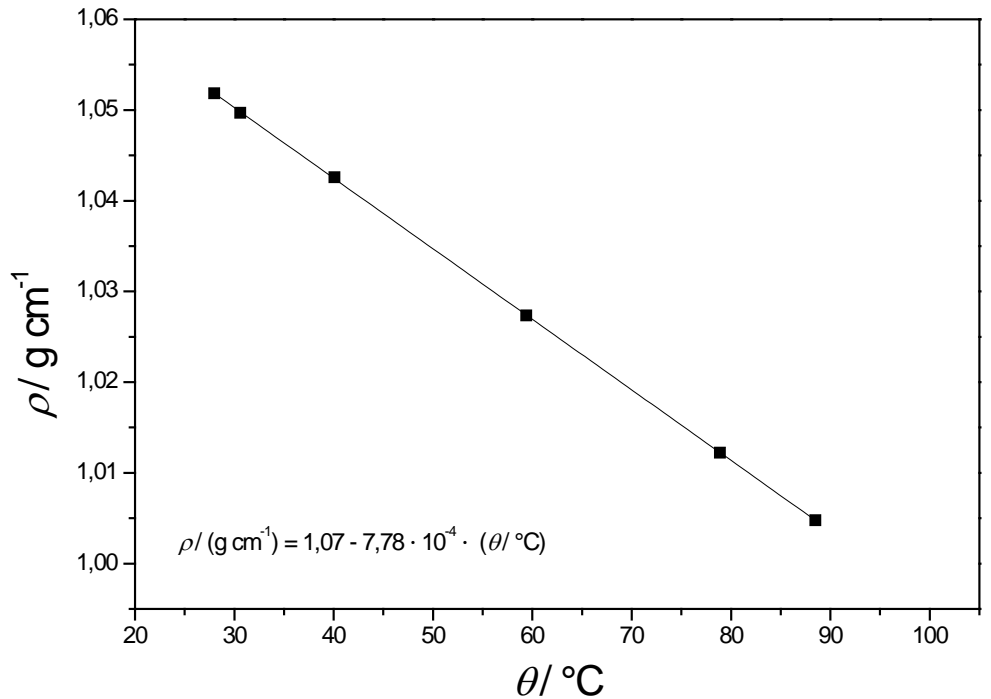

Abbildung 12.4: Dichte als Funktion der Temperatur für MA-HPBCHC (10 wt \%) in BL-087.

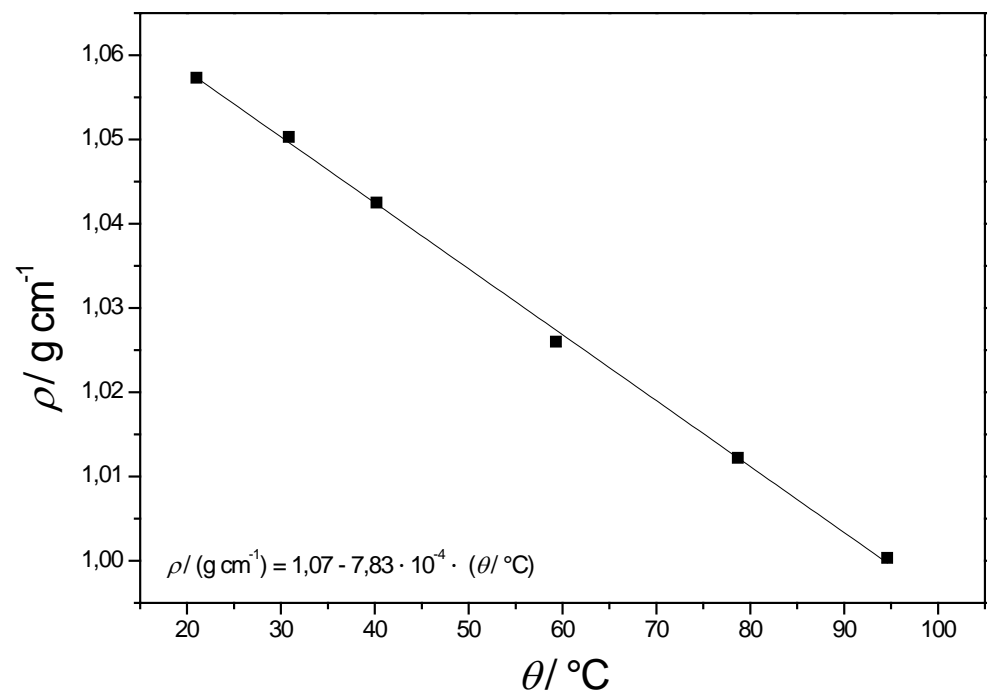

Abbildung 12.5: Dichte als Funktion der Temperatur für MA-HPBCHC (10 wt \%) in BL-087 mit R-5011 (5 wt\%). 


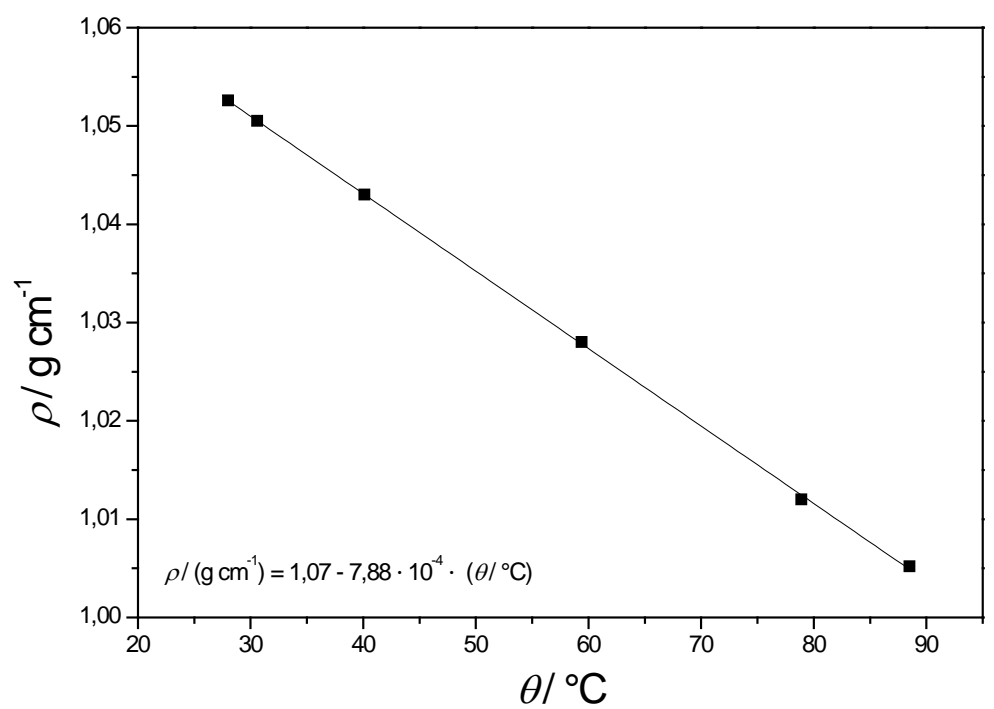

Abbildung 12.6: Dichte als Funktion der Temperatur für MA-HPBCHC (10 wt\%) in BL-087 mit R-5011 (7 wt\%).

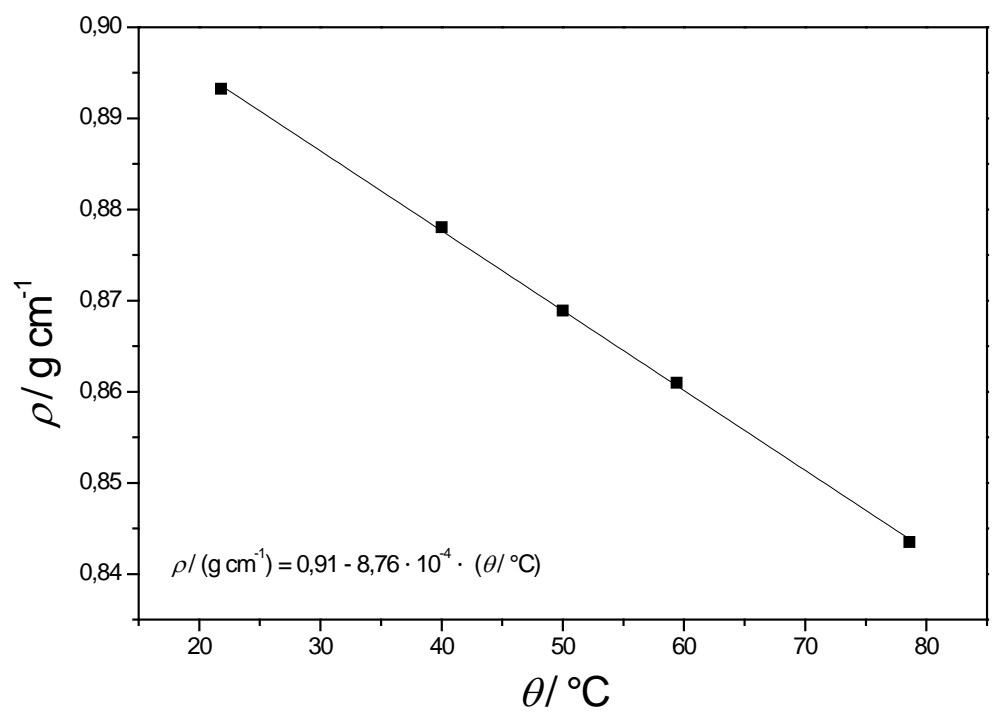

Abbildung 12.7: Dichte als Funktion der Temperatur für MA-HPBCHC (20 wt\%) in Toluol. 


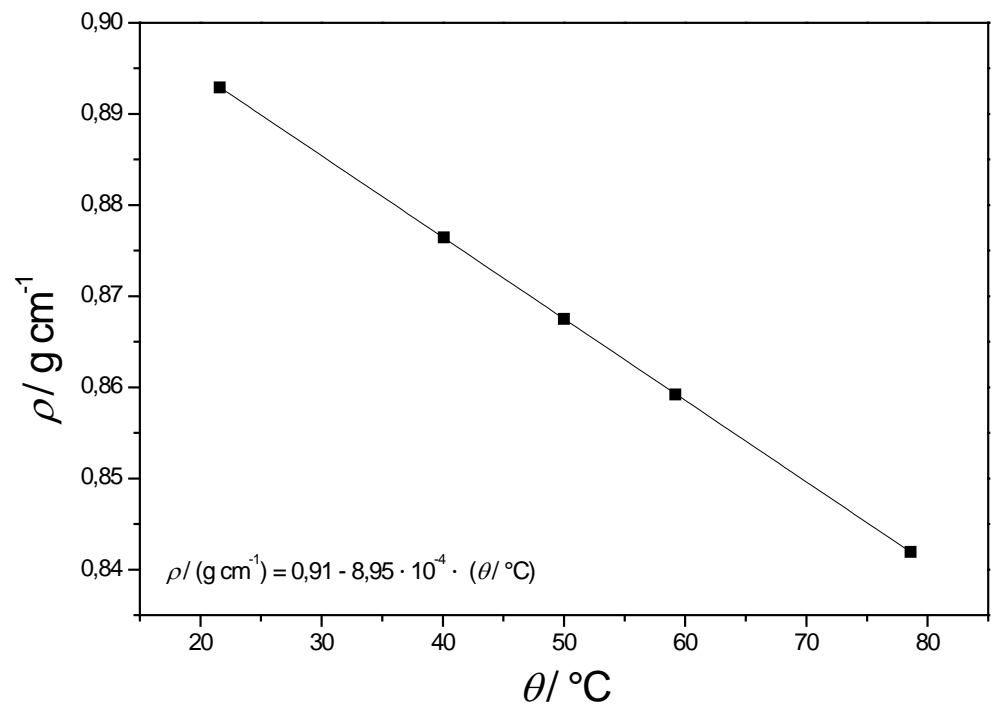

Abbildung 12.8: Dichte als Funktion der Temperatur für A-HPBCHC (25 wt \%) in Toluol.

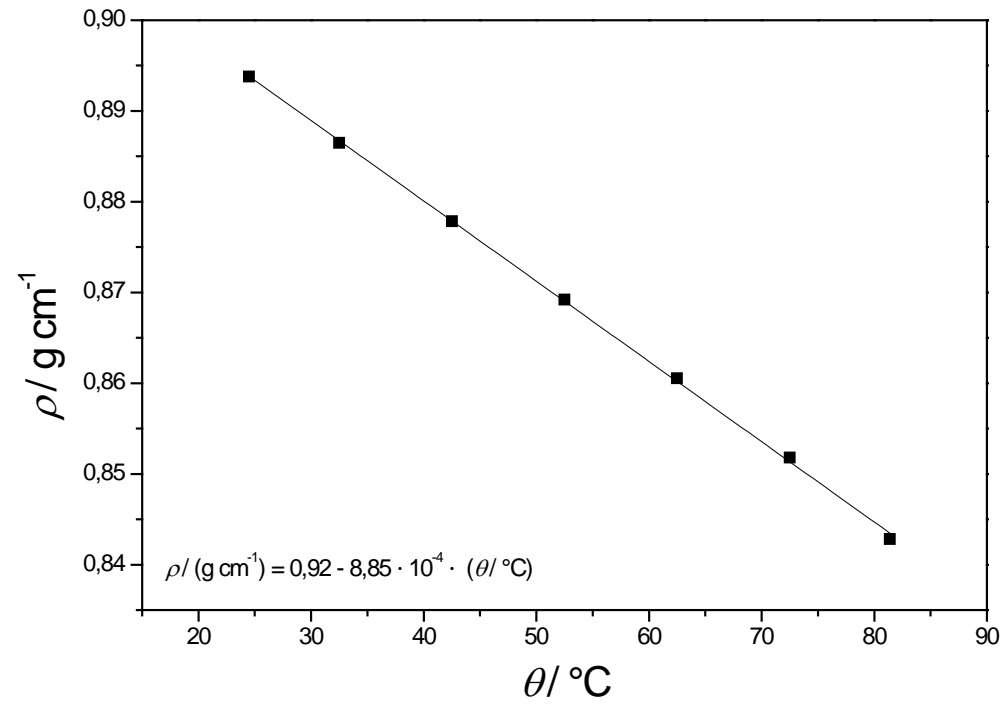

Abbildung 12.9: Dichte als Funktion der Temperatur für FA-HPBCHC (20 wt\%) in Toluol. 


\subsection{PLP-SEC Ergebnisse}

Tabelle 12.1: Reaktionsbedingungen und Ergebnisse der PLP-SEC Experimente von MA-HPBCHC.

\begin{tabular}{|c|c|c|c|c|}
\hline $\mathrm{T} /{ }^{\circ} \mathrm{C}$ & $c_{\text {Ini }} / \mathrm{mmol} \mathrm{L}^{-1}$ & $\begin{array}{c}v_{\text {rep }} / \\
\mathrm{Hz}\end{array}$ & $M_{1} / M_{2}$ & $k_{\mathrm{p}} / \mathrm{L} \mathrm{mol}^{-1} \mathrm{~s}^{-1}$ \\
\hline 20 & 10 & 4 & 0,58 & 315 \\
\hline 20 & 10 & 5 & 0,52 & 338 \\
\hline 20 & 10 & 7 & 0,47 & 352 \\
\hline 20 & 50 & 3 & 0,56 & 307 \\
\hline 20 & 50 & 4 & 0,58 & 318 \\
\hline 20 & 50 & 5 & 0,55 & 330 \\
\hline 20 & 50 & 6 & 0,54 & 336 \\
\hline 20 & 50 & 7 & 0,49 & 348 \\
\hline 40 & 10 & 8 & 0,59 & 576 \\
\hline 40 & 10 & 10 & 0,52 & 610 \\
\hline 40 & 10 & 12 & 0,48 & 628 \\
\hline 40 & 50 & 7 & 0,58 & 568 \\
\hline 40 & 50 & 8 & 0,58 & 585 \\
\hline 40 & 50 & 9 & 0,57 & 602 \\
\hline 40 & 50 & 10 & 0,52 & 603 \\
\hline 40 & 50 & 11 & 0,54 & 637 \\
\hline 40 & 50 & 12 & 0,49 & 651 \\
\hline
\end{tabular}




\begin{tabular}{|c|c|c|c|c|}
\hline$T /{ }^{\circ} \mathrm{C}$ & $c_{\text {Ini }} / \mathrm{mmol} \mathrm{L}^{-1}$ & $\begin{array}{c}v_{\text {rep }} / \\
\mathrm{Hz}\end{array}$ & $M_{1} / M_{2}$ & $k_{\mathrm{p}} / \mathrm{L} \mathrm{mol}^{-1} \mathrm{~s}^{-1}$ \\
\hline 60 & 10 & 15 & 0,58 & 990 \\
\hline 60 & 50 & 10 & 0,59 & 980 \\
\hline 60 & 50 & 12 & 0,57 & 981 \\
\hline 60 & 50 & 13 & 0,57 & 1006 \\
\hline 60 & 50 & 14 & 0,57 & 1016 \\
\hline 60 & 50 & 15 & 0,56 & 1017 \\
\hline 60 & 50 & 16 & 0,56 & 1030 \\
\hline 60 & 50 & 17 & 0,56 & 1035 \\
\hline 60 & 50 & 18 & 0,56 & 1039 \\
\hline 60 & 50 & 20 & 0,46 & 1031 \\
\hline 60 & 100 & 15 & 0,56 & 1019 \\
\hline 80 & 10 & 30 & 0,48 & 1615 \\
\hline 80 & 50 & 22 & 0,58 & 1563 \\
\hline 80 & 50 & 23 & 0,57 & 1587 \\
\hline 80 & 50 & 24 & 0,57 & 1596 \\
\hline 80 & 50 & 25 & 0,57 & 1593 \\
\hline 80 & 50 & 26 & 0,55 & 1631 \\
\hline 80 & 50 & 27 & 0,55 & 1638 \\
\hline 80 & 50 & 28 & 0,55 & 1649 \\
\hline 80 & 100 & 20 & 0,56 & 1676 \\
\hline 80 & 100 & 30 & 0,47 & 1581 \\
\hline
\end{tabular}


Tabelle 12.2: Reaktionsbedingungen und Ergebnisse der PLP-SEC Experimente von A-HPBCHC.

\begin{tabular}{|c|c|c|c|c|}
\hline$T /{ }^{\circ} \mathrm{C}$ & $c_{\text {Ini }} / \mathrm{mmol} \mathrm{L}^{-1}$ & $\begin{array}{c}v_{\text {rep }} / \\
\mathrm{Hz}\end{array}$ & $M_{1} / M_{2}$ & $k_{\mathrm{p}} / \mathrm{L} \mathrm{mol}^{-1} \mathrm{~s}^{-1}$ \\
\hline 0,7 & 50 & 200 & 0,50 & 6108 \\
\hline 0,7 & 50 & 150 & 0,60 & 6504 \\
\hline 0,7 & 50 & 100 & 0,60 & 5531 \\
\hline 0,7 & 50 & 50 & 0,57 & 4193 \\
\hline 0,7 & 50 & 25 & 0,54 & 3199 \\
\hline 0,7 & 50 & 10 & - & 1197 \\
\hline 0,7 & 50 & 5 & - & 614 \\
\hline 0,7 & 50 & 2 & - & 200 \\
\hline 0,7 & 100 & 200 & 0,54 & 6560 \\
\hline 11,4 & 50 & 200 & 0,60 & 8485 \\
\hline 11,4 & 50 & 175 & 0,62 & 8338 \\
\hline 11,4 & 50 & 150 & 0,63 & 8547 \\
\hline 11,4 & 50 & 125 & 0,59 & 6966 \\
\hline 11,4 & 50 & 100 & 0,60 & 6834 \\
\hline 11,4 & 50 & 75 & 0,58 & 5805 \\
\hline 11,4 & 50 & 50 & - & 4845 \\
\hline 11,4 & 50 & 25 & - & 2394 \\
\hline
\end{tabular}




\begin{tabular}{|c|c|c|c|c|}
\hline$T /{ }^{\circ} \mathrm{C}$ & $c_{\text {Ini }} / \mathrm{mmol} \mathrm{L}^{-1}$ & $\begin{array}{c}v_{\text {rep }} / \\
\mathrm{Hz}\end{array}$ & $M_{1} / M_{2}$ & $k_{\mathrm{p}} / \mathrm{L} \mathrm{mol}^{-1} \mathrm{~s}^{-1}$ \\
\hline 11,4 & 50 & 10 & - & 1123 \\
\hline 11,4 & 50 & 8 & - & 922 \\
\hline 11,4 & 50 & 6 & - & 661 \\
\hline 11,4 & 100 & 200 & 0,53 & 8397 \\
\hline 20,4 & 50 & 400 & 0,50 & 12652 \\
\hline 20,4 & 50 & 300 & 0,58 & 12725 \\
\hline 20,4 & 50 & 250 & 0,59 & 10602 \\
\hline 20,4 & 50 & 200 & 0,59 & 11541 \\
\hline 20,4 & 50 & 150 & 0,65 & 11644 \\
\hline 20,4 & 50 & 100 & 0,67 & 9205 \\
\hline 20,4 & 50 & 50 & 0,52 & 5530 \\
\hline 20,4 & 50 & 25 & - & 2256 \\
\hline 20,4 & 50 & 10 & - & 1126 \\
\hline 20,4 & 50 & 5 & - & 613 \\
\hline 20,4 & 100 & 400 & 0,54 & 11887 \\
\hline 30,3 & 50 & 400 & 0,54 & 14031 \\
\hline 30,3 & 50 & 350 & 0,58 & 14620 \\
\hline 30,3 & 50 & 300 & 0,55 & 14325 \\
\hline 30,3 & 50 & 275 & 0,62 & 13968 \\
\hline 30,3 & 50 & 200 & 0,60 & 11372 \\
\hline 30,3 & 50 & 100 & 0,55 & 8290 \\
\hline
\end{tabular}




\begin{tabular}{|c|c|c|c|c|}
\hline$T /{ }^{\circ} \mathrm{C}$ & $c_{\text {Ini }} / \mathrm{mmol} \mathrm{L}^{-1}$ & $\begin{array}{c}v_{\text {rep }} / \\
\mathrm{Hz}\end{array}$ & $M_{1} / M_{2}$ & $k_{\mathrm{p}} / \mathrm{L} \mathrm{mol}^{-1} \mathrm{~s}^{-1}$ \\
\hline 30,3 & 50 & 75 & 0,38 & 5613 \\
\hline 30,3 & 50 & 50 & 0,26 & 4179 \\
\hline 30,3 & 50 & 25 & 0,25 & 2384 \\
\hline 30,3 & 50 & 10 & 0,22 & 1156 \\
\hline 30,3 & 50 & 5 & 0,21 & 631 \\
\hline 30,3 & 100 & 400 & 0,56 & 13950 \\
\hline 50,7 & 50 & 700 & 0,59 & 22979 \\
\hline 50,7 & 50 & 600 & 0,58 & 23116 \\
\hline 50,7 & 50 & 500 & 0,63 & 24813 \\
\hline 50,7 & 50 & 400 & 0,62 & 21346 \\
\hline 50,7 & 50 & 300 & 0,57 & 18261 \\
\hline 50,7 & 50 & 200 & 0,49 & 13420 \\
\hline 50,7 & 50 & 100 & - & 6545 \\
\hline 50,7 & 50 & 75 & - & 5115 \\
\hline 50,7 & 50 & 50 & - & 3624 \\
\hline 50,7 & 50 & 25 & - & 2060 \\
\hline 50,7 & 50 & 10 & - & 995 \\
\hline 50,7 & 100 & 700 & 0,52 & 23643 \\
\hline 69,1 & 50 & 900 & 0,54 & 29887 \\
\hline 69,1 & 50 & 800 & 0,55 & 29401 \\
\hline 69,1 & 50 & 600 & 0,48 & 23888 \\
\hline
\end{tabular}




\begin{tabular}{ccccc}
\hline$T /{ }^{\circ} \mathrm{C}$ & $c_{\text {Ini }} / \mathrm{mmol} \mathrm{L}^{-1}$ & $\begin{array}{c}v_{\mathrm{rep}} / \\
\mathrm{Hz}\end{array}$ & $M_{1} / M_{2}$ & $k_{\mathrm{p}} / \mathrm{L} \mathrm{mol}^{-1} \mathrm{~s}^{-1}$ \\
\hline 69,1 & 50 & 400 & 0,36 & 13931 \\
69,1 & 50 & 200 & - & 7428 \\
69,1 & 50 & 100 & - & 4427 \\
69,1 & 50 & 50 & - & 2617 \\
69,1 & 50 & 25 & - & 1533 \\
69,1 & 100 & 800 & 0,54 & 29643 \\
\hline
\end{tabular}


Tabelle 12.3: Reaktionsbedingungen und Ergebnisse der PLP-SEC Experimente von FA-HPBCHC.

\begin{tabular}{|c|c|c|c|c|}
\hline $\mathrm{T} /{ }^{\circ} \mathrm{C}$ & $c_{\text {Ini }} / \mathrm{mmol} \mathrm{L}^{-1}$ & $\begin{array}{c}v_{\text {rep }} / \\
\mathrm{Hz}\end{array}$ & $M_{1} / M_{2}$ & $k_{\mathrm{p}} / \mathrm{L} \mathrm{mol}^{-1} \mathrm{~s}^{-1}$ \\
\hline 20,6 & 4 & 45 & 0,57 & 6448 \\
\hline 20,6 & 4 & 50 & 0,54 & 6288 \\
\hline 20,6 & 4 & 55 & 0,54 & 6184 \\
\hline 20,6 & 15 & 55 & 0,53 & 6400 \\
\hline 40,9 & 15 & 60 & 0,59 & 10660 \\
\hline 40,9 & 15 & 70 & 0,57 & 10724 \\
\hline 40,9 & 15 & 80 & 0,54 & 10688 \\
\hline 40,9 & 15 & 100 & 0,49 & 10487 \\
\hline 59,7 & 15 & 120 & 0,57 & 17690 \\
\hline 59,7 & 15 & 140 & 0,53 & 1772 \\
\hline 59,7 & 15 & 160 & 0,52 & 17946 \\
\hline 59,7 & 4 & 120 & 0,57 & 17542 \\
\hline 79,1 & 15 & 160 & 0,60 & 28263 \\
\hline 79,1 & 15 & 200 & 0,53 & 26832 \\
\hline 79,1 & 15 & 220 & 0,51 & 26199 \\
\hline 79,1 & 15 & 160 & 0,59 & 26719 \\
\hline 79,1 & 15 & 180 & 0,58 & 27500 \\
\hline
\end{tabular}




\subsection{Abkürzüngsverzeichnis}

A

$a$

$\alpha$

$\alpha_{1}$

$\alpha_{\mathrm{s}}$

B

CR

BL-087

$c_{\mathrm{I}}$

$c_{\mathrm{M}}$

$c_{\mathrm{M}}^{0}$

$c_{\mathrm{M}}(t)$

$c_{\mathrm{r}}$

$c_{\mathrm{R}}^{0}$

DMPA

EHMA

ESR

$E_{\mathrm{A}}$

FT

f

HTRL

K präexponentieller Faktor

Mark-Houwink-Parameter

Koeffizient der Kettenlängenabhängigkeit

Koeffizient der Kettenlängenabhängigkeit im

Langkettenbereich

Koeffizient der Kettenlängenabhängigkeit im

Kurzkettenbereich

Magnetfeldstärke

chemische Reaktion

Nematischer Flüssigkristall von Merck KGaA

Initiatorkonzentration

Monomerkonzentration

Startmonomerkonzentration

Monomerkonzentration zum Zeitpunkt t

Radikalkonzentration

Startradikalkonzentration

2,2-Dimethoxy-2-phenyl-acetophenon

Ethylhexylmethacrylat

Elektronen-Spin-Resonanz

Aktivierungsenergie

Fourier-Transformation

Initiatoreffektivität

High-termination Rate Limit

Mark-Houwink-Parameter 


\begin{tabular}{|c|c|}
\hline$\eta$ & Viskosität \\
\hline$\eta_{0}$ & Viskosität bei 0 \% Umsatz \\
\hline$\eta_{\mathrm{r}}$ & relative Viskosität \\
\hline IR & Infrarot \\
\hline IUPAC & International Union of Pure and Applied Chemistry \\
\hline$i, j$ & Polymerkettenlängen \\
\hline$i_{\mathrm{c}}$ & crossover-Kettenlänge \\
\hline $\begin{array}{l}k_{\mathrm{bb}} \\
\text { Reaktion }\end{array}$ & Geschwindigkeitskoeffizient der \\
\hline$k_{\mathrm{d}}$ & Zerfallsgeschwindigkeitskoeffizient des Initiators \\
\hline$k_{\mathrm{i}}$ & $\begin{array}{lcc}\text { Geschwindigkeitskoeffizient } \quad \text { der } & \text { ersten } \\
\text { Monomeraddition nach dem Initiatorzerfall } & \end{array}$ \\
\hline$k_{\mathrm{p}}$ & $k_{\mathrm{p}}$ ohne diffusionskontrollierten Anteil \\
\hline$k_{\mathrm{p}, \mathrm{D}}$ & diffusionskontrollierter Anteil an $k_{\mathrm{p}}$ \\
\hline$k_{\mathrm{t}}$ & Terminierungsgeschwindigkeitskoeffizient \\
\hline$\left\langle k_{\mathrm{t}}\right\rangle$ & $\begin{array}{l}\text { kettenlängengemittelter } \\
\text { Terminierungsgeschwindigkeitskoeffizient }\end{array}$ \\
\hline$k_{\mathrm{t}}^{i, j}$ & $\begin{array}{l}\text { Terminierungsgeschwindigkeitskoeffizient } \quad \text { zweier } \\
\text { Radikale der Kettenlänge } i \text { und } j\end{array}$ \\
\hline$k_{\mathrm{t}}^{i, i}$ & $\begin{array}{l}\text { Terminierungsgeschwindigkeitskoeffizient zweier } \\
\text { gleichlanger Radikale der Kettenlänge } i\end{array}$ \\
\hline$k_{\mathrm{t}}^{1,1}$ & $\begin{array}{l}\text { Terminierungsgeschwindigkeitskoeffizient } \quad \text { zweier } \\
\text { Radikale der Kettenlänge } 1\end{array}$ \\
\hline$k_{\mathrm{TD}}$ & $\begin{array}{l}\text { Anteil der Translationsdiffusion } \\
\text { Terminierungskoeffizienten }\end{array}$ \\
\hline$k_{\mathrm{SD}}$ & $\begin{array}{l}\text { Anteil der Segmentdiffusion } \\
\text { Terminierungskoeffizienten }\end{array}$ \\
\hline
\end{tabular}




\begin{tabular}{|c|c|}
\hline$k_{\mathrm{CR}}$ & $\begin{array}{l}\text { Anteil der chemischen } \\
\text { Terminierungskoeffizienten }\end{array}$ \\
\hline$k_{\mathrm{RD}}$ & $\begin{array}{l}\text { Anteil der } \quad \text { Reaktionsdiffusion } \\
\text { Terminierungskoeffizienten }\end{array}$ \\
\hline$k_{t r}$ & Geschwindigkeitskoeffizient der Transferreaktion \\
\hline$L_{b}$ & Polymerisationsgrad \\
\hline$l$ & Schichtlänge \\
\hline LTRL & Low-termination Rate Limit \\
\hline$\lambda$ & Wellenlänge \\
\hline$m$ & Masse \\
\hline MCR & mid-chain radical \\
\hline $\bar{M}_{n}$ & Zahlenmittel der Molmassenverteilung \\
\hline $\bar{M}_{\mathrm{w}}$ & Gewichtsmittel der Molmassenverteilung \\
\hline NIR & Nahinfrarot \\
\hline$N_{A}$ & Avogadro-Konstante \\
\hline POI & Point of inflection \\
\hline PLP-SEC & $\begin{array}{l}\text { Pulsed-Laser-Polymerization-Size } \\
\text { Chromatography }\end{array}$ \\
\hline R-5011 & Chiraler Dotierstoff von Merck KGaA \\
\hline RAFT & Reversible Addition-Fragmentation Chain-Transfer \\
\hline $\mathrm{RD}$ & Reaktionsdiffusion \\
\hline$R$ & ideale Gaskonstante \\
\hline ( & Registered Trade Mark \\
\hline$\rho$ & Dichte \\
\hline SD & Segmentdiffusion \\
\hline SEC & Size-Exclusion-Chromatography \\
\hline SP-PLP & Einzelpuls-Pulslaser-Polymerisation \\
\hline
\end{tabular}




$\begin{array}{ll}\text { SP-PLP-ESR } & \begin{array}{l}\text { Einzelpuls-Pulslaser-Polymerisation } \\ \text { Kombination mit ESR-Spektroskopie }\end{array} \\ \text { SPR } & \text { Sekündäres propagierendes Radikal } \\ \Phi & \text { Primärquantenausbeute } \\ \text { TD } & \text { Translationsdiffusion } \\ T & \text { absolute Temperatur } \\ \text { UV } & \text { Ultraviolett } \\ V & \text { Volumen } \\ v_{\text {rep }} & \text { Laserpulsfrequenz } \\ \text { wt } \% & \text { Gewichtsprozent } \\ X & \text { Umsatz }\end{array}$




\section{Literaturverzeichnis}

[1] W. J. HORN, A. SOEGANTO, F. M. SHAIKH, AIAA Journal 1989, 27, 1399-1405.

[2] O. F. Olaj, I. Bitai, Angew. Makromol. Chemie 1987, 155, 177-190.

[3] O. F. Olaj, I. Bitai, F. Hinkelmann, Makromol. Chem. 1987, 188, 1689-1702.

[4] M. Buback, R. G. Gilbert, R. A. Hutchinson, B. Klumperman, F.-D. Kuchta, B. G. Manders, K. F. O'Driscoll, G. T. Russell, J. Schweer, Macromol. Chem. Phys. 1995, 196, 3267-3280.

[5] S. Beuermann, M. Buback, T. P. Davis, R. G. Gilbert, R. A. Hutchinson, O. F. Olaj, G. T. Russell, J. Schweer, van Herk, Alex M., Macromol. Chem. Phys. 1997, 198, 1545-1560.

[6] S. Beuermann, M. Buback, T. P. Davis, R. G. Gilbert, R. A. Hutchinson, A. Kajiwara, B. Klumperman, G. T. Russell, Macromol. Chem. Phys. 2000, 201, 1355-1364.

[7] C. Barner-Kowollik, S. Beuermann, M. Buback, P. Castignolles, B. Charleux, M. L. Coote, R. A. Hutchinson, T. Junkers, I. Lacík, G. T. Russell et al., Polym. Chem. 2014, 5, 204-212.

[8] J. M. Asua, S. Beuermann, M. Buback, P. Castignolles, B. Charleux, R. G. Gilbert, R. A. Hutchinson, J. R. Leiza, A. N. Nikitin, J.-P. Vairon et al., Macromol. Chem. Phys. 2004, 205, 2151-2160.

[9] I. Lacík, S. Beuermann, M. Buback, Macromolecules 2003, 36, 9355-9363.

[10] I. Lacík, L. Učňová, S. Kukučková, M. Buback, P. Hesse, S. Beuermann, Macromolecules 2009, 42, 7753-7761. 
[11] S. Beuermann, M. Buback, P. Hesse, F.-D. Kuchta, I. Lacík, van Herk, Alex M., Pure and Applied Chemistry 2007, 79, 1080.

[12] P. Drawe, M. Buback, Macromol. Theory Simul. 2016, 25, 74-84.

[13] M. Buback, M. Egorov, T. Junkers, E. Panchenko, Macromol. Rapid Commun. 2004, 25, 1004-1009.

[14] M. Buback, M. Egorov, T. Junkers, E. Panchenko, Macromol. Chem. Phys. 2005, 206, 333-341.

[15] A. Kajiwara, K. Maeda, N. Kubo, M. Kamachi, Macromolecules 2003, 36, 526-528.

[16] A. Kajiwara, Macromol. Symp. 2007, 248, 50-59.

[17] J. Barth, M. Buback, P. Hesse, T. Sergeeva, Macromolecules 2010, 43, 4023-4031.

[18] J. Barth, W. Meiser, M. Buback, Macromolecules 2012, 45, 13391345.

[19] H. Kattner, M. Buback, Macromolecules 2015, 48, 7410-7419.

[20] E. M. Barrall, II, J. F. Johnson, Journal of Macromolecular Science, Part C 1979, 17, 137-170.

[21] H. Kikuchi, M. Yokota, Y. Hisakado, H. Yang, T. Kajiyama, Nat Mater 2002, 1, 64-68.

[22] S. G. Kim, S. M. Kim, Y. S. Kim, H. K. Lee, S. H. Lee, G.-D. Lee, J.-J. Lyu, K. H. Kim, Appl. Phys. Lett. 2007, 90, 261910.

[23] S. M. Kim, Y. in Cho, W. I. Kim, K.-U. Jeong, S. H. Lee, G.-D. Lee, J. Son, J.-J. Lyu, K. H. Kim, Japanese Journal of Applied Physics 2009, 48, 32405.

[24] T. Iwata, K. Suzuki, N. Amaya, H. Higuchi, H. Masunaga, S. Sasaki, H. Kikuchi, Macromolecules 2009, 42, 2002-2008.

[25] C. A. Guymon, Science 1997, 275, 57-59.

[26] C. A. Guymon, C. N. Bowman, Macromolecules 1997, 30, 52715278.

[27] C. A. Guymon, C. N. Bowman, Macromolecules 1997, 30, 15941600.

[28] M. Buback, R. G. Gilbert, G. T. Russell, D. J. T. Hill, G. Moad, K. F. O'Driscoll, J. Shen, M. A. Winnik, J. Polym. Sci. A Polym. Chem. 1992, 30, 851-863.

[29] M. Buback, Makromol. Chem. 1990, 191, 1575-1587.

[30] M. v. Smoluchowski, Zeitschrift für Physikalische Chemie 1918, $92 U$. 
[31] T. G. Carswell, D.J.T. Hill, D. I. Londero, J. H. O'Donnell, P. J. Pomery, C. L. Winzor, Polymer 1992, 33, 137-140.

[32] H. Yamazoe, P. B. Zetterlund, B. Yamada, D. J. T. Hill, P. J. Pomery, Macromol. Chem. Phys. 2001, 202, 824-829.

[33] D. S. Achilias, Macromol. Theory Simul. 2007, 16, 319-347.

[34] H. Kattner, M. Buback, Macromolecules 2015, 48, 309-315.

[35] M. Buback, F.-D. Kuchta, Macromol. Chem. Phys. 1997, 198, 14551480 .

[36] S. W. Benson, A. M. North, J. Am. Chem. Soc. 1962, 84, 935-940.

[37] Pascal Hesse, Dissertation, Georg-August-Universität, Göttingen, 2008.

[38] V. E. Trommsdorff, H. Köhle, P. Lagally, Makromol. Chem. 1948, 1, 169-198.

[39] Willemse, Robin X. E., B. B. P. Staal, van Herk, Alex M., S. C. J. Pierik, B. Klumperman, Macromolecules 2003, 36, 9797-9803.

[40] O. F. Olaj, P. Vana, M. Zoder, A. Kornherr, G. Zifferer, Macromol. Rapid Commun. 2000, 21, 913-920.

[41] J. P.A. Heuts, G. T. Russell, European Polymer Journal 2006, 42, $3-$ 20.

[42] G. B. Smith, G. T. Russell, J. P.A. Heuts, Macromol. Theory Simul. 2003, 12, 299-314.

[43] J. Barth, M. Buback, Macromolecules 2011, 44, 1292-1297.

[44] M. Buback, M. Egorov, T. Junkers, E. Panchenko, Macromol. Rapid Commun. 2004, 25, 1004-1009.

[45] Nicolai Sörensen, Dissertation, Georg-August-Universität, Göttingen, 2015.

[46] G. T. Russell, R. G. Gilbert, D. H. Napper, Macromolecules 1993, 26, 3538-3552.

[47] D. Cuccato, E. Mavroudakis, M. Dossi, D. Moscatelli, Macromol. Theory Simul. 2013, 22, 127-135.

[48] X. Yu, L. J. Broadbelt, Macromol. Theory Simul. 2012, 21, 461-469.

[49] T. Junkers, C. Barner-Kowollik, J. Polym. Sci. A Polym. Chem. 2008, 46, 7585-7605.

[50] A. N. Nikitin, R. A. Hutchinson, Macromolecules 2005, 38, 15811590.

[51] A. N. Nikitin, R. A. Hutchinson, Macromol. Theory Simul. 2006, 15, 128-136. 
[52] A. N. Nikitin, R. A. Hutchinson, Macromolecular rapid communications 2009, 30, 1981-1988.

[53] A. N. Nikitin, R. A. Hutchinson, G. A. Kalfas, J. R. Richards, C. Bruni, Macromol. Theory Simul. 2009, 18, 247-258.

[54] S. Beuermann, M. Buback, Progress in Polymer Science 2002, 27, 191-254.

[55] S. Beuermann, Macromolecules 2002, 35, 9300-9305.

[56] B. Dervaux, T. Junkers, M. Schneider-Baumann, F. E. Du Prez, C. Barner-Kowollik, J. Polym. Sci. A Polym. Chem. 2009, 47, 66416654.

[57] T. Junkers, M. Schneider-Baumann, S. S. P. Koo, P. Castignolles, C. Barner-Kowollik, Macromolecules 2010, 43, 10427-10434.

[58] Z. Grubisic, P. Rempp, H. Benoit, J. Polym. Sci. B Polym. Lett. 1967, 5, 753-759.

[59] M. Buback, E. Müller, G. T. Russell, J. Phys. Chem. A 2006, 110, 3222-3230.

[60] G. B. Smith, G. T. Russell, Zeitschrift für Physikalische Chemie 2005, 219, 295-323.

[61] D. R. Lide, CRC handbook of chemistry and physics. A readyreference book of chemical and physical data, 76. Aufl., CRC Press, [S.l.], 1997.

[62] B. Otazaghine, B. Boutevin, P. Lacroix-Desmazes, Macromolecules 2002, 35, 7634-7641.

[63] Y. Luo, A.-N. Wang, X. Gao, Soft Matter 2012, 8, 1824-1830.

[64] B. Hojjati, P. A. Charpentier, J. Polym. Sci. A Polym. Chem. 2008, 46, 3926-3937.

[65] G. Moad, Y. K. Chong, A. Postma, E. Rizzardo, S. H. Thang, Polymer 2005, 46, 8458-8468.

[66] J. T. Lai, D. Filla, R. Shea, Macromolecules 2002, 35, 6754-6756.

[67] D.-i. Choi, E. H. Yeom, M. Park, J. K. Kim, B. C. Kim, J. Appl. Polym. Sci. 2004, 93, 2082-2089.

[68] J.-M. Cracowski, V. Montembault, D. Bosc, B. Améduri, F. Odobel, L. Fontaine, J. Polym. Sci. A Polym. Chem. 2009, 47, 1403-1411.

[69] K. Skrabania, A. Miasnikova, A. M. Bivigou-Koumba, D. Zehm, A. Laschewsky, Polym. Chem. 2011, 2, 2074.

[70] J. Brandrup, Polymer handbook, 4. Aufl., Wiley, New York [u.a.], 1999. 
[71] N. V. Tsarevsky, B. S. Sumerlin, Fundamentals of Controlled/Living Radical Polymerization, Royal Society of Chemistry, Cambridge, 2013.

[72] S. Beuermann, D. A. Paquet, J. H. McMinn, R. A. Hutchinson, Macromolecules 1996, 29, 4206-4215.

[73] van Herk, Alex M., J. Chem. Educ. 1995, 72, 138.

[74] A. P. Haehnel, M. Schneider-Baumann, K. U. Hiltebrandt, A. M. Misske, C. Barner-Kowollik, Macromolecules 2013, 46, 15-28.

[75] G. E. Roberts, T. P. Davis, J. P. A. Heuts, G. E. Ball, Macromolecules 2002, 35, 9954-9963.

[76] S. Beuermann, Pure and Applied Chemistry 2003, 75, 1091.

[77] R. A. Hutchinson, S. Beuermann, D. A. Paquet, J. H. McMinn, C. Jackson, Macromolecules 1998, 31, 1542-1547.

[78] R. A. Hutchinson, S. Beuermann, D. A. Paquet, J. H. McMinn, Macromolecules 1997, 30, 3490-3493.

[79] S. Beuermann, M. Buback, P. Hesse, I. Lacík, Macromolecules 2006, 39, 184-193.

[80] N. M. Ahmad, F. Heatley, P. A. Lovell, Macromolecules 1998, 31, 2822-2827.

[81] A. P. Haehnel, M. Schneider-Baumann, L. Arens, A. M. Misske, F. Fleischhaker, C. Barner-Kowollik, Macromolecules 2014, 47, 34833496.

[82] R. X. E. Willemse, A. M. van Herk, Macromol. Chem. Phys. 2010, 211, 539-545.

[83] M. Buback, C. H. Kurz, C. Schmaltz, Macromol. Chem. Phys. 1998, 199, 1721-1727.

[84] J. P. A. Heuts, R. G. Gilbert, L. Radom, Macromolecules 1995, 28, 8771-8781.

[85] A. N. Nikitin, R. A. Hutchinson, M. Buback, P. Hesse, Macromolecules 2007, 40, 8631-8641.

[86] B. Wenn, T. Junkers, Macromolecular rapid communications 2016 , 37, 781-787.

[87] J. Barth, M. Buback, P. Hesse, T. Sergeeva, Macromolecular rapid communications 2009, 30, 1969-1974.

[88] H. Kattner, M. Buback, Macromolecular rapid communications 2015, 36, 2186-2191. 
[89] A. Kajiwara, A. K. Nanda, K. Matyjaszewski, Macromolecules 2004, 37, 1378-1385.

[90] A. Kajiwara, M. Kamachi in ACS Symposium Series, Vol. 854 (Hrsg.: K. Matyjaszewski), American Chemical Society, Washington, DC, 2003.

[91] M. Azukizawa, B. Yamada, D. J. T. Hill, P. J. Pomery, Macromol. Chem. Phys. 2000, 201, 774-781.

[92] B. Yamada, M. Azukizawa, H. Yamazoe, D. J. T. Hill, P. J. Pomery, Polymer 2000, 41, 5611-5618.

[93] E. Sato, T. Emoto, P. B. Zetterlund, B. Yamada, Macromol. Chem. Phys. 2004, 205, 1829-1839.

[94] M. Buback, P. Hesse, T. Junkers, T. Sergeeva, T. Theis, Macromolecules 2008, 41, 288-291.

[95] L. S. Boguslavskaya, I. Y. Panteleeva, T. V. Morozova, A. V. Kartashov, N. N. Chuvatkin, Russ. Chem. Rev. 1990, 59, 906.

[96] B. Yamada, T. Kontani, M. Yoshioka, T. Otsu, J. Polym. Sci. Polym. Chem. Ed. 1984, 22, 2381-2393.

[97] R. W. Fessenden, R. H. Schuler, J. Chem. Phys. 1965, 43, 2704 2712.

[98] P. J. Krusic, R. C. Bingham, J. Am. Chem. Soc. 1976, 98, 230-232.

[99] T. Junkers, D. Voll, C. Barner-Kowollik 2009, 9.

[100] M. Ueda, S. Shouji, T. Ogata, M. Kamachi, C. U. Pittman, Macromolecules 1984, 17, 2800-2804.

[101] S. Kobatake, B. Yamada, Polym J 1996, 28, 535-542.

[102] J. Penelle, J. Collot, G. Rufflard, J. Polym. Sci. A Polym. Chem. 1993, 31, 2407-2412.

[103] M. Kamachi, A. Kajiwara, Macromolecules 1996, 29, 2378-2382.

[104] M. P. Tonge, A. Kajiwara, M. Kamachi, R. G. Gilbert, Polymer 1998, 39, 2305-2313.

[105] M. Kamachi in Polymer Physics, Springer Berlin Heidelberg, Berlin, Heidelberg, 1987.

[106] L. Hermosilla, P. Calle, C. Sieiro, N. García, P. Tiemblo, J. Guzmán, Chemical Physics 2007, 340, 237-244.

[107] H. Kattner, P. Drawe, M. Buback, Macromol. Chem. Phys. 2015, 216, 1737-1745.

[108] L. E. Redington, J. Polym. Sci. 1948, 3, 503-517. 
[109] J. Barth, M. Buback, Macromolecular Reaction Engineering 2010, 4, 288-301.

[110] R. A. Hutchinson, S. Beuermann, D. A. Paquet, J. H. McMinn, Macromolecules 1997, 30, 3490-3493.

[111] J. Barth, M. Buback, P. Hesse, T. Sergeeva, Macromolecules 2009, 42, 481-488.

[112] H. Tanaka, K. Kuwata, Bull. Chem. Soc. Jpn. 1978, 51, 2451-2455.

[113] M. I. Boamfa, S. V. Lazarenko, E. C. M. Vermolen, A. Kirilyuk, T. Rasing, Adv. Mater. 2005, 17, 610-614.

[114] H.R. Falle, G.R. Luckhurst, Journal of Magnetic Resonance (1969) 1970, 3, 161-199.

[115] O. H. Griffith, D. W. Cornell, H. M. McConnell, J. Chem. Phys. 1965, 43, 2909-2910.

[116] J. Barth, M. Buback, P. Hesse, T. Sergeeva, Macromolecules 2009, 42, 481-488.

[117] M. Buback, E. Müller, G. T. Russell, J. Phys. Chem. A 2006, 110, 3222-3230.

[118] H. Kattner, M. Buback, Macromolecules 2015, 48, 309-315.

[119] J. Barth, M. Buback, P. Hesse, T. Sergeeva, Macromolecular rapid communications 2009, 30, 1969-1974.

[120] J. Barth, M. Buback, G. T. Russell, S. Smolne, Macromol. Chem. Phys. 2011, 212, 1366-1378.

[121] B. Friedman, B. O'Shaughnessy, Macromolecules 1993, 26, 57265739.

[122] J. Barth, M. Buback, Macromolecular rapid communications 2009, 30, 1805-1811.

[123] M. Strukelj, J. M. G. Martinho, M. A. Winnik, R. P. Quirk, Macromolecules 1991, 24, 2488-2492.

[124] A. R. Khokhlov, Makromol. Chem., Rapid Commun. 1981, 2, 633636.

[125] G. Johnston-Hall, A. Theis, M. J. Monteiro, T. P. Davis, M. H. Stenzel, C. Barner-Kowollik, Macromol. Chem. Phys. 2005, 206, 2047-2053.

[126] S. Smolne, S. Weber, M. Buback, Macromol. Chem. Phys. 2016, 217, 2391-2401.

[127] H. Hervet, W. Urbach, F. Rondelez, J. Chem. Phys. 1978, 68, 2725. 
[128] T. Turiv, I. Lazo, A. Brodin, B. I. Lev, V. Reiffenrath, V. G.

Nazarenko, O. D. Lavrentovich, Science 2013, 342, 1351-1354.

[129] C. Gähwiller, Molecular Crystals and Liquid Crystals 2007, 20, 301318.

[130] K.-S. CHU, D. S. MOROI, J. Phys. Colloques 1975, 36, C1-99-C1101.

[131] W. Franklin, Phys. Rev. A 1975, 11, 2156-2164.

[132] J. A. Murphy, J. W. Doane, Molecular Crystals and Liquid Crystals 2007, 13, 93-95.

[133] H. Hakemi, M. M. Labes, J. Chem. Phys. 1975, 63, 3708.

[134] J. Barth, M. Buback, Macromolecular rapid communications 2009, 30, 1805-1811.

[135] Kim, Cho, Palffy-Muhoray, Kyu, Physical review letters 1993, 71, 2232-2235.

[136] D.-K. Yang, L.-C. Chien, J. W. Doane, Appl. Phys. Lett. 1992, 60, 3102. 


\section{Danksagung}

Mein besonderer und herzlicher Dank gilt Herrn Prof. Dr. Philipp Vana, MBA für das anspruchsvolle Thema und die Unterstützung im Laufe der Jahre. Ich bin überzeugt, dass mich die Zeit in der Abteilung Makromolekulare Chemie in meiner persönlichen und fachlichen Entwicklung enorm vorangetrieben hat.

Ich möchte herzlich Herrn Prof. Dr. Martin Suhm für die Übernahme des Koreferats danken.

Weiterhin bedanke ich mich bei Herrn Prof. Dr. Ricardo Mata, Prof. Dr. Dietmar Stalke, PD Dr. Thomas Zeuch und Dr. Florian Ehlers für die Teilnahme am Prüfungskomitee.

I want to thank Dr. Atsushi Kajiwara for taking me into his group in Nara, Japan and teaching me a lot about ESR. I really enjoyed visiting your beautiful home country!

Special thanks to Dr. David Wilkes at Merck KGaA for all the great discussions and the possibilities to present my work.

Ich möchte in einem Zug all den Mitarbeitern der Arbeitsgruppen Vana und Buback für die großartige Arbeitsatmosphäre danken. Ich kann hier nicht ausreichend erwähnen was mir die Freundschaft mit jedem einzelnen von euch bedeutet. 


\section{Biografie des Autors}

Alex Groschopp geboren am 07.02.1989 in Buenos Aires, Argentinien

Deutsche Staatsangehörigkeit

11/2014-02/2018 Georg-August-Universität Göttingen

Institut für Physikalische Chemie

Abteilung Makromolekulare Chemie

Promotion, Chemie

„Kinetik der radikalischen Polymerisation von

Monomeren mit mesogener Seitengruppe in isotroper und anisotroper Lösung"

Betreuer: Prof. Dr. Philipp Vana, MBA

05/2017-07/2017 Nara University of Education, Japan

Arbeitsgruppe von Prof. Dr. Atsushi Kajiwara

Forschungsaufenthalt

09/2012 - 10/2014 Technische Universität Braunschweig

Master of Science, Chemie

Masterarbeit: „Anbindung von Polymeren mit Aminogruppen an Elektroden für Mikrobielle Brennstoffzellen"

Betreuer: Prof. Dr. Menzel

Abschlussnote: 1,2

09/2009-08/2012 Technische Universität Braunschweig

Bachelor of Science, Chemie

Masterarbeit: „Die Synthese enantiomerenreiner

a-Methylcarbonsäuren durch Enolat-Alkylierung“

Betreuer: Prof. Dr. Schulz

Abschlussnote: 1,3

04/2002 - 08/2008 Gymnasium Fallersleben

Abitur

Abschlussnote: 1,7 
